\title{
Untersuchung von \\ Einzel- und Mehrblasensystemen in akustischen Resonatoren
}

\author{
Dissertation \\ zur Erlangung des Doktorgrades \\ der Mathematisch-Naturwissenschaftlichen Fakultäten \\ der Georg-August-Universität zu Göttingen \\ vorgelegt von \\ Dagmar Krefting \\ aus Münster
}

Göttingen 2003 
D7

Referent: Prof. Dr. Werner Lauterborn

Korreferent: Prof. Dr. Dirk Ronneberger

Tag der mündlichen Prüfung: 


\section{Inhaltsverzeichnis}

1 Einleitung 3

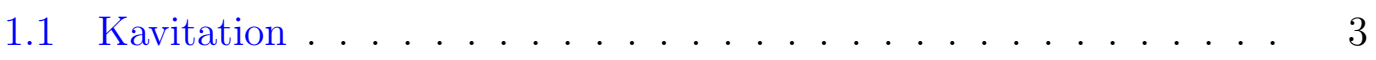

1.2 Ziel und Aufbau der Arbeit . . . . . . . . . . . . . . 7

2 Theoretische Grundlagen 9

2.1 Radialdynamik ........................ 9

2.2 Translationsdynamik . . . . . . . . . . . . . . . . . . . . 12

2.3 Diffusionsprozesse . . . . . . . . . . . . . . . . . . 18

2.4 Oberflächeninstabilitäten . . . . . . . . . . . . . . . 21

2.5 Wechselwirkung benachbarter Blasen . . . . . . . . . . . . 23

3 Die Mittelung der linearisierten Bewegungsgleichung 25

4 Experimenteller Aufbau und Messmethoden 33

4.1 Erzeugung des Schallfeldes . . . . . . . . . . . . . . . 34

4.2 Bildaufnahme . . . . . . . . . . . . . . . . 35

4.3 Aufnahme des Schallsignals . . . . . . . . . . . . 36

$\begin{array}{lll}5 & \text { Die Einzelblase } & 37\end{array}$

5.1 Rückkopplung der Blase auf den Resonator . . . . . . . . . . . 37

5.2 Translation ........................ 46

5.3 Oberflächenschwingungen . . . . . . . . . . . . 56

5.4 SBSL in luftgesättigtem Wasser . . . . . . . . . . . . 62 
$\begin{array}{llr}6 & \text { Die Clusterstruktur } & 69\end{array}$

$\begin{array}{lll}7 & \text { Streamerstrukturen } & 77\end{array}$

7.1 Eindimensionaler Streamer . . . . . . . . . . . . . . . 78

7.2 Zweidimensionale Streamerstrukturen . . . . . . . . . . . . 86

$\begin{array}{lll}8 & \text { Jellyfish-Strukturen } & 91\end{array}$

8.1 Die Jellyfish-Struktur . . . . . . . . . . . . . . . . . . . . . . 91

8.2 Die Starfish-Struktur - Blasenstruktur nahe der Wasseroberfläche 101

9 Akustisches Kavitationsspektrum und räumliche Strukturbildung 107

9.1 Modulation der Amplitude mit einem Dreiecksignal . . . . . . . . 107

9.2 Reorganisation des Schallfeldes nach dem Umrühren . . . . . . . . 121

9.3 Blasenstruktur an einer Befestigungsschraube . . . . . . . . . . 123

$\begin{array}{ll}10 \text { Ultraschallreinigung und Kavitationserosion } & 125\end{array}$

10.1 Reinigung von Glasplatten . . . . . . . . . . . . . . . . . 125

10.2 Erosion von Aluminiumfolie . . . . . . . . . . . . . . . 130

10.3 Kavitationserosion an Weißblech . . . . . . . . . . . . . 131

11 Zusammenfassung 133

12 Ausblick 135

$\begin{array}{ll}\text { A Beschreibung der verwendeten Resonatoren } & 139\end{array}$

A.1 Glaswürfel . . . . . . . . . . . . . . . . . . . . . . 139

A.2 Plexiglasquader . . . . . . . . . . . . . . . . . . . 149

A.3 Plexiglaswanne . . . . . . . . . . . . . . . . . . . . . 151

A.4 Ultraschallreaktor . . . . . . . . . . . . . . . . . . . . 152

$\begin{array}{ll}\text { B Symbole } & 155\end{array}$ 


\section{Kapitel 1}

\section{Einleitung}

\subsection{Kavitation}

Kavitation bezeichnet die Hohlraumbildung in Flüssigkeiten. Bei starker Zugspannung reißt die Flüssigkeitsstruktur an Störstellen auf, es bilden sich Kavitationsblasen. Beim Aufschwingen dieser Hohlräume können sowohl Flüssigkeitsdampf als auch gelöste Gase in die Blase gelangen. Der Inhalt einer Kavitationsblase hängt wesentlich von den Zeitskalen ab, auf denen der Hohlraum gebildet wird und Verdampfungs- bzw. Diffusionsprozesse stattfinden. Auch durch fokussierten Energieeintrag kann das Aufreißen der Flüssigkeit induziert werden. Dann führt ein lokaler Überdruck zum Aufschwingen einer Blase, die zusätzlich Produkte aus Plasmabildung enthalten kann. In Abbildung 1.1 ist die schematische Einteilung der Kavitation nach Lauterborn dargestellt [63].

Unabhängig von ihrer Entstehung folgen die Blasen den gleichen physikalischen Prinzipien. Dominiert nach dem Aufschwingen der Außendruck über den Innendruck der Blase, so kollabiert sie. Ist der Druckunterschied groß, so wird dieser Kollaps sehr heftig, die Blasenwand erreicht Überschallgeschwindigkeit und eine Stoßwelle wird in die Flüssigkeit ausgestrahlt. In der Blase verbleibendes Gas wird stark komprimiert, gleichzeitig wird ein kurzer Lichtblitz ausgestrahlt. Dieses Phänomen wird Kavitationslumineszenz genannt. Die Anwesenheit einer Grenzfläche in der Nähe der Blase führt zu einem unsymmetrischen Kollaps, der in der Ausbildung eines Flüssigkeitsjets resultieren kann. Die Oberfläche der Grenzfläche wird angegriffen. Verschmutzungen auf der Oberfläche werden in akustischen Blasenfeldern gereinigt. Kavitation kann aber auch zu erheblicher Materialschädigung führen, der sogenannten Kavitationserosion.

Die Untersuchung all dieser Phänomene ist Gegenstand der Kavitationsforschung $[52,100,12]$. 


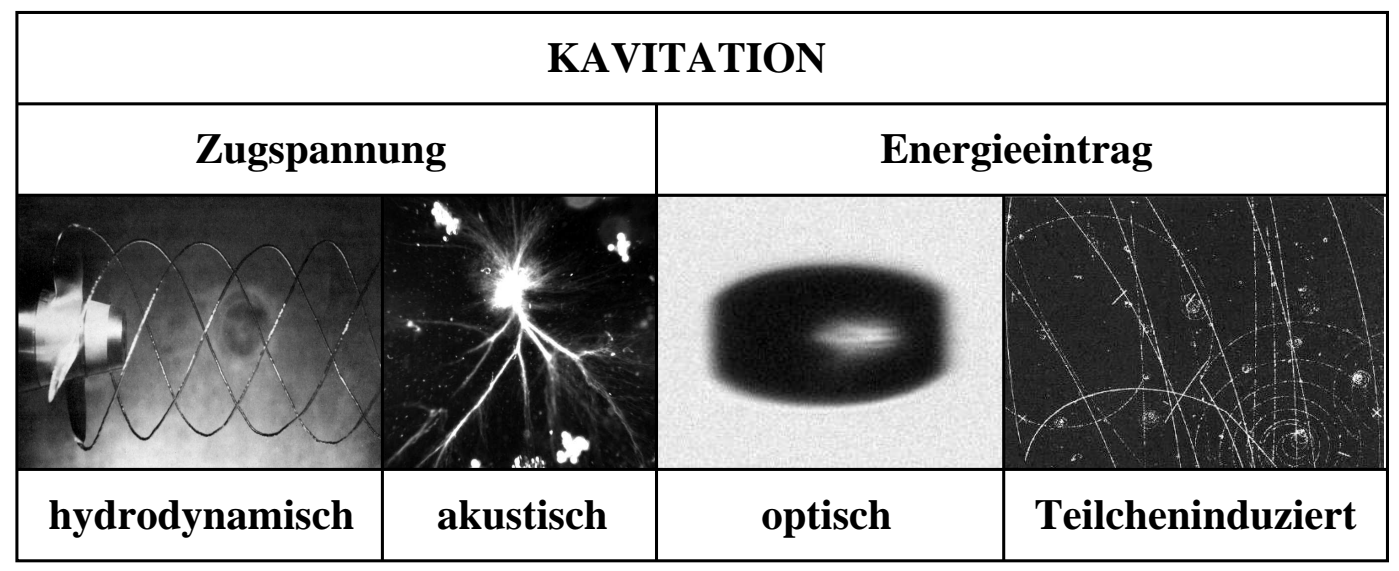

Abbildung 1.1: Klassifizierung der Kavitation [63]. Abbildungen aus $[34,36,11]$.

\section{Akustische Kavitation}

In dieser Arbeit liegt der Schwerpunkt auf der akustischen Kavitation [66]. Durch Schallwellen werden Kavitationsblasen zu Volumenschwingungen angeregt, die stark nichtlinear werden können. Der Schalldruckgradient in Stehwellenfeldern induziert eine Translationsbewegung der Blase relativ zur Flüssigkeit. Die pulsierenden Blasen strahlen ihrerseits Schallwellen aus. Damit beeinflussen sie das äußere Schallfeld, üben aber vor allem Kräfte auf benachbarte Blasen aus. Diese können miteinander kollidieren, durch Oberflächenschwingungen können Blasen fragmentiert und Mikroblasen abgespalten werden. All diese Phänomene führen in akustischen Kavitationsfeldern zu komplexen raumzeitlichen Strukturen, den sogenannten Kavitationsstrukturen.

\section{Kavitationsforschung}

Kavitation rückte zuerst als technischer Störfaktor ins Interesse der Naturwissenschaften. Als man Ende des 19. Jahrhunderts Schiffe mit hohem Vortrieb entwickelte, stellte man fest, dass in der Praxis die Schiffsschraube ein Blasenfeld erzeugte, das den Wirkungsgrad des Schiffsmotors drastisch reduzierte. Auch die Schädigung der Schiffsschraube konnte mit der Entstehung von Kavitation in Verbindung gebracht werden. Bereits 1902 veröffentlichte Vilhelm Bjerknes die Arbeiten seines Vaters über die hydrodynamischen Kräfte einer oszillierenden Flüssigkeit auf eine pulsierende Kugel und die Kräfte zwischen zwei pulsierenden Kugeln [9]. 1917 stellte Rayleigh die Bewegungsgleichung eines kollabierenden sphärischen Hohlraums in einer Flüssigkeit auf [69]. 


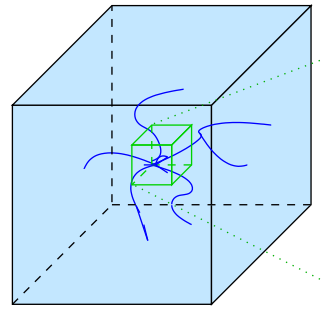

$10 \mathrm{~cm}$

Orientierung

der Struktur

im Schallfeld

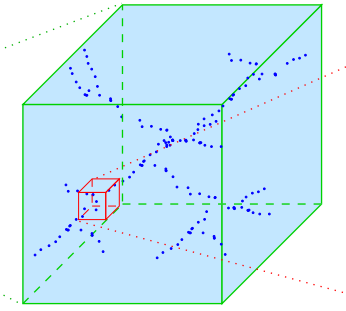

$1 \mathrm{~cm}$

Orientierung

der Blasen

in der Struktur

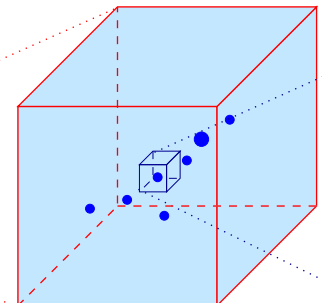

$1 \mathrm{~mm}$

Translation

Kollision

Clusterbildung

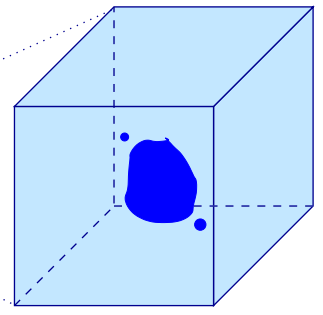

$10 \mu \mathrm{m}$

Pulsation

Oberflächenmoden

Fragmentierung

Abbildung 1.2: Relevante Raumskalen in der akustischen Kavitation.

Zeitgleich entwickelte Langevin leistungsstarke piezoelektrische Ultraschallsender, die eine intensive Forschung über die Effekte und Anwendungsmöglichkeiten von Ultraschall in Gang setzten. 1927 beschrieben Wood und Loomis zahlreiche typische Ultraschalleffekte, unter anderem die innere Erwärmung von Stoffen, Emulgation, Koagulation und die Fähigkeit, Glas zu bohren [7, 57]. Damit waren sowohl die theoretischen als auch die experimentellen Grundlagen der akustischen Kavitation geschaffen.

In der Ultraschalltechnik waren es wieder die unerwünschten Effekte von Kavitation, diesmal bei Hochleistungssendern in der Sonartechnik, die die Forschung an akustischer Kavitation aus militärischer Sicht notwendig machte.

Auch die Ultraschallreinigung wurde zu Beginn für militärische Zwecke entwickelt, 1939 erhielt Heimberger ein Deutsches Reichspatent auf ein Verfahren zur Reinigung von Metallflächen, (DRP Nr. 733470). Zehn Jahre später folgte Fruth mit einem amerikanischen Patent (Method of and apparatus for cleaning by ultrasound waves, (Am. pat. Nr. 2468550). Nach Kriegsende, als eine stürmische Entwicklung auf allen Gebieten der Ultraschalltechnik einsetzte, gelangte die Reinigung schnell zur technischen Reife und ist heute neben der Materialprüfung die Hauptanwendung von Ultraschall im zivilen Bereich. Industriell wird die Ultraschallreinigung insbesondere in der Feinmechanik und der Optik verwendet, aber auch medizinische Instrumente, Schmuck und elektrische Bauteile werden mit Ultraschall gereinigt.

Die theoretische Beschreibung von Kavitation beschränkte sich in den fünfziger und sechziger Jahren im Wesentlichen auf die Dynamik von Einzelblasen. Phänomene wie Lumineszenz oder das Auftreten von Subharmonischen der Anregungsfrequenz in der Schallemission wurden zwar als Kavitationseffekte erkannt, konnten aber nicht erklärt werden. 


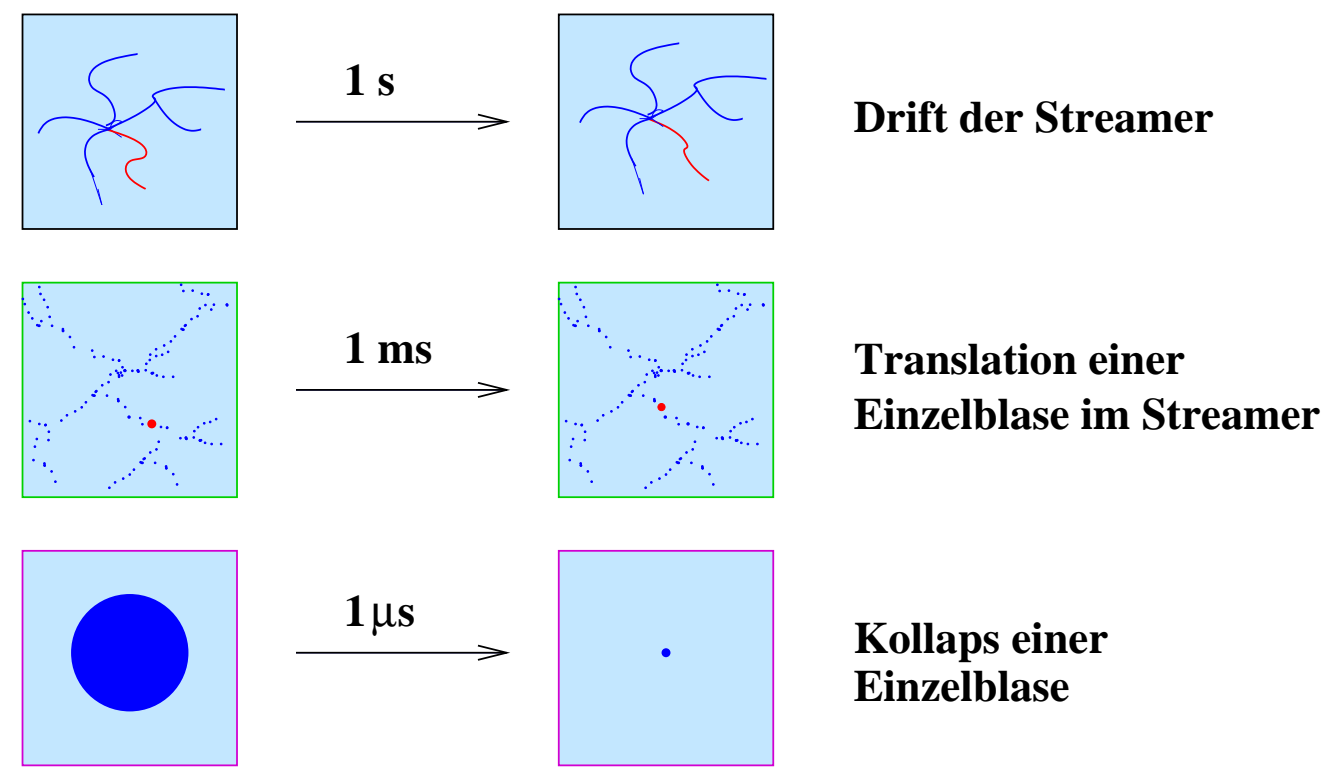

Abbildung 1.3: Relevante Zeitskalen in der akustischen Kavitation.

Die Entwicklung der laserinduzierten Kavitation und die Entdeckung der Einzelblasensonolumineszenz (SBSL) ermöglichten die Untersuchung von isolierten, heftig kollabierenden Blasen und schufen so neue Einblicke in die Natur der Lumineszenz [4, 61, 32]. Die Behandlung der Blase als nichtlinearer getriebener Oszillator zeigte bei hoher Schallanregung ein vielfältiges Resonanzspektrum und konnte sowohl das Breitbandrauschen als auch das Linienspektrum im akustischen Signal von Kavitationsfeldern erklären. Untersuchungen an Grenzflächen konnten einen Zusammenhang zwischen Blasendynamik und Erosionserscheinungen zeigen. Generell war aber die Beschreibung der akustischen Kavitationsfelder eher phänomenologischer Natur. Das Wissen über die Entstehung und das Verhalten von Blasenstrukturen ist immer noch lückenhaft. Das hat mehrere Gründe. Die nichtlinearen Wechselwirkungsprozesse im Kavitationsfeld schließen eine einfache Superposition der einzelnen Blasen aus. Das System ist dissipativ und weder die Anzahl noch das Volumen der Blasen ist konstant. Die große Anzahl der Blasen macht eine numerische Simulation rechenaufwendig, insbesondere wenn das Strömungsfeld der Flüssigkeit mitmodelliert werden muss. Die physikalischen Prozesse spielen sich auf Zeit- und Raumskalen ab, die mehrere Größenordnungen auseinanderliegen, aber nicht entkoppelt werden können. Entscheidende Prozesse liegen außerhalb der derzeit technisch möglichen Auflösung.

Dies führt zwangsläufig dazu, dass der experimentelle Beweis vieler Theorien noch nicht erbracht werden konnte, auch wenn die Phänomene schon seit langem bekannt sind. 


\subsection{Ziel und Aufbau der Arbeit}

Trotz der Komplexität der Kavitationsfelder scheint die Anzahl der auftretenden Blasenstrukturen begrenzt zu sein. In unterschiedlichen Ultraschallresonatoren werden wiederkehrende charakteristische Strukturen beobachtet, die bei geringfügiger Änderung eines Parameters oft sprunghaft ineinander übergehen. Ziel dieser Arbeit ist die Beschreibung, Charakterisierung und Erklärung der auftretenden Blasenstrukturen in akustischen Kavitationsfeldern.

$\mathrm{Zu}$ Beginn der Arbeit werden die theoretischen Grundlagen zusammengestellt, die für das Verständnis der Arbeit notwendig sind. Die allgemeinen Bewegungsgleichungen der Blasenwand und des Blasenmittelpunktes einer Einzelblase bilden die theoretische Basis der hydrodynamischen Betrachtung der Kavitationsfelder, Diffusion und Oberflächenschwingungen beschreiben wesentliche Faktoren für die Instabilität von Kavitationsblasen.

Es folgt eine analytische Betrachtung der Gültigkeit vereinfachter zeitlicher Mittelung bei der Translation schwach schwingender Blasen.

Dem experimentellen Teil der Arbeit wird eine allgemeine Beschreibung der Messapparatur und der Messmethoden vorangestellt.

Der Hauptteil der Arbeit, die Beschreibung der Kavitationsfelder, ist in die verschiedenen Blasenstrukturen aufgeteilt. Die Reihenfolge wurde dabei nach zunehmender Komplexität der Kavitationsstruktur gewählt. Zuerst werden Untersuchungen an Einzelblasensystemen vorgestellt und verschiedene Aspekte ihrer Dynamik beleuchtet. Es folgt die Charakterisierung der Clusterstruktur, die als langlebige Struktur oberhalb des Stabilitätsbereiches der Einzelblase gefunden wurde und sich in gewissen Aspekten wie eine kollektive Gesamtblase verhält. Daran schließt sich die Untersuchung der Streamerstruktur an, die in Anwesenheit von Blasenquellen im Schalldruckbereich von Einzelblasensonolumineszenz und Clusterbildung existent ist. Die Beschreibung der Kavitationsstrukturen im Flüssigkeitsvolumen schließt mit der Jellyfish-Struktur, die in sehr starken Schallfeldern entsteht.

Die Untersuchung des Kavitationsspektrums erstreckt sich über einen weiten Schalldruckbereich und beschäftigt sich mit dem Zusammenhang zwischen subharmonischen Anteilen im akustischen Spektrum und der Entstehung verschiedener Blasenstrukturen. In diesem Zusammenhang wird auch eine Blasenstruktur an der Grenzfläche des Resonators behandelt, die sich nicht in das Schema der bisher vorgestellten Strukturen einfügen lässt.

Im letzten Kapitel des experimentellen Teils wird die Dynamik von Blasenstrukturen in Anwesenheit einer Grenzfläche untersucht. Dabei liegt der Schwerpunkt auf Reinigungs- und Erosionsprozessen im Kavitationsblasenfeld. 
Ein zusammenfassendes Kapitel setzt die einzelnen Abschnitte der Arbeit strukturübergreifend in Beziehung zueinander. Zum Abschluss dieser Arbeit werden auf Grundlage der gefundenen Ergebnisse Möglichkeiten weiterführender Untersuchungen zum besseren Verständnis akustischer Kavitationsblasenfelder diskutiert.

Der Anhang enthält die Beschreibung der verwendeten akustischen Resonatoren. Variablen werden im Text in der Regel nur bei ihrer Einführung erläutert und sind zur Übersicht im Symbolverzeichnis aufgelistet. 


\section{Kapitel 2}

\section{Theoretische Grundlagen}

\subsection{Radialdynamik}

\subsubsection{Die Rayleigh-Plesset-Gleichung}

Die Bewegungsgleichung einer sphärischen Blase in einer idealen, rein radial strömenden, inkompressiblen Flüssigkeit an der Blasenwand lässt sich durch die sogenannte Rayleigh-Plesset-Gleichung beschreiben [8, 69, 58, 84]

$$
\rho\left(R \ddot{R}+\frac{3}{2} \dot{R}^{2}\right)=p_{R}-p_{\infty}
$$

mit

$$
\begin{aligned}
& \rho: \text { Dichte der Flüssigkeit, } \\
& R: \text { Blasenradius, } \\
& p_{R}: \text { Blaseninnendruck, } \\
& p_{\infty}: \text { Außendruck. }
\end{aligned}
$$

\subsubsection{Erweiterungen}

Die obige Grundgleichung ist in verschiedenen Näherungen weiterentwickelt und hergeleitet worden:

\section{RPNNP-Modell}

Die Rayleigh-Plesset-Neppiras-Noltingk-Poritsky-Gleichung (RPNNP-Modell) wurde für eine Blase im Schallfeld aufgestellt und berücksichtigt Oberflächenspannung und Viskosität der Flüssigkeit, sowie eine konstante Menge idealen Gases als Blasenfüllung [80, 88] 


$$
\begin{aligned}
R \ddot{R}+\frac{3}{2} \dot{R}^{2} & =\frac{1}{\rho}\left[\left(p_{h s}+\frac{2 \sigma}{R_{0}}\right)\left(\frac{R_{0}}{R}\right)^{3 \gamma}+p_{v}-\frac{2 \sigma}{R}-\frac{4 \eta \dot{R}}{R}-p_{h s}-p_{a} \cos (\omega t)\right] \\
& =\frac{p_{l}}{\rho}
\end{aligned}
$$

mit

$p_{h s}$ : Hydrostatischer Druck in der Flüssigkeit an der Stelle der Blase,

$\sigma$ : Oberflächenspannung der Flüssigkeit,

$R_{0}$ : Gleichgewichtsradius bei gegebenem Innendruck,

$\gamma$ : Polytropenexponent des Füllgases,

$p_{v}$ : Dampfdruck der Flüssigkeit,

$\eta$ : Dynamische Viskosität der Flüssigkeit,

$p_{a}$ : Schalldruckamplitude am Ort der Blase,

$\omega$ : Frequenz des anregenden Schallfeldes.

\section{Keller-Miksis-Modell}

Die Keller-Miksis-Gleichung erweitert die RPNNP-Gleichung um die Kompressibilität der umgebenden Flüssigkeit [49]

$$
\left(1-\frac{\dot{R}}{C}\right) R \ddot{R}+\frac{3}{2} \dot{R}^{2}\left(1-\frac{\dot{R}}{3 C}\right)=\left(1+\frac{\dot{R}}{C}\right) \frac{p_{l}}{\rho} \frac{R}{\rho C} \frac{\mathrm{d} p_{l}}{\mathrm{~d} t}
$$

mit

$$
C \text { : Schallgeschwindigkeit. }
$$

\section{Gilmore-Modell}

Die Gilmore-Gleichung berücksichtigt die Dichteabhängigkeit der Schallgeschwindigkeit und lässt Überschallgeschwindigkeiten der Blasenwand und damit Stoßwellenentwicklung zu [38]

$$
R \ddot{R}\left(1-\frac{\dot{R}}{C}\right)+\left(3-\frac{\dot{R}}{C}\right) \frac{\dot{R}^{2}}{2}=\left(1-\frac{\dot{R}}{C}\right) \frac{R \dot{R}}{C} \frac{d H}{d R}+\left(1+\frac{\dot{R}}{C}\right) H
$$

mit

$$
H=\int_{p \infty}^{p_{l}} \frac{d p}{\rho}
$$


Qualitativ geben alle Modelle die Radialschwingungen von oszillierenden Blasen gut wieder. Wesentliche Unterschiede zeigen sich erst in der Modellierung des sehr heftigen Kollapses bei hohen Schalldruckamplituden.

\subsubsection{Die linearisierte Radialgleichung}

Bei niedrigen Schalldruckamplituden kann die Pulsation linear genähert

$$
R(t)=R_{0}+R_{a t}(t)
$$

und die Blase als harmonischer Oszillator beschrieben werden. Die lineare RadialGleichung

$$
4 \pi R_{0} \rho \ddot{R}_{a t}+16 \pi \eta R_{0} \dot{R}_{a t}+4 \pi R_{0}\left[3 \kappa\left(p_{0}+\frac{2 \sigma}{R_{0}}\right)-\frac{2 \sigma}{R_{0}}\right] R_{a t}=-4 \pi R_{0}^{2} p_{a} \sin (\omega t)
$$

entspricht der linearisierten RPNNP-Gleichung [66]. Die Blase führt harmonische Radialschwingungen der Anregungsfrequenz $\omega$ um den Ruheradius aus

$$
R(t)=R_{0}-R_{a} \sin (\omega t+\phi),
$$

die Eigenfrequenz $\omega_{0}$ der Blase bei gegebenem Ruheradius $R_{0}$ ist

$$
\omega_{0}^{2}=\frac{1}{R_{0}^{2} \rho}\left[3 \kappa\left(p_{h s}+\frac{2 \sigma}{R_{0}}\right)-\frac{2 \sigma}{R_{0}}-\frac{4 \eta^{2}}{R_{0}^{2} \rho}\right] .
$$

Damit ergeben sich Schwingungsamplitude $R_{a}$ und Phasenverschiebung $\phi$ zu [37]

$$
R_{a}=\frac{p_{a}}{\sqrt{\left(\rho R_{0}\right)^{2}\left(\omega_{0}^{2}-\omega^{2}\right)^{2}+\left(\frac{4 \eta}{R_{0}}\right)^{2} \omega^{2}}}, \quad \phi=-\arctan \left(\frac{4 \eta \omega}{\rho\left(\omega_{0}^{2}-\omega^{2}\right)}\right) .
$$

Für Luftblasen in Wasser hat Minnaert eine einfache Näherung für die Relation zwischen Resonanzradius $R_{r}$ und Resonanzfrequenz aufgestellt, die sogenannte Minnaertfrequenz [78]

$$
\omega_{0} \approx \frac{6 \pi}{R_{r}} \quad \frac{\mathrm{m}}{\mathrm{s}}
$$

Damit können wir unter Vernachlässigung des Reibungstermes einen einfachen Ausdruck für die Auslenkung finden

$$
R_{a}=\frac{R_{0} p_{a}}{36 \rho \pi^{2}\left[1-\left(\frac{R_{0}}{R_{r}}\right)^{2}\right]}
$$




\subsection{Translationsdynamik}

\subsubsection{Die Bewegungsgleichung}

Bei gleichem Impulsübertrag auf eine Blase und ein gleich großes Flüssigkeitsvolumen wird die Blase aufgrund der geringeren Masse stärker beschleunigt. Dies führt zu einer Relativgeschwindigkeit $\dot{x}_{B}$ der Blase gegenüber der Flüssigkeitsbewegung $\dot{x}_{l}$. Bei einer Relativbewegung muss Flüssigkeit verdrängt, also ebenfalls beschleunigt werden. Die Translationsbewegung in einer inkompressiblen Flüssigkeit wird durch folgende Bewegungsgleichung beschrieben ${ }^{1}[9,72]$

$$
\frac{d}{d t} P_{B}=\rho V_{B} \ddot{x}_{l}-\frac{d}{d t} P_{l}\left(V_{B}\right),
$$

mit

$P_{B}:$ Impuls der Blase,

$V_{B}$ : Volumen der Blase,

$\dot{x}_{l}$ : Geschwindigkeit der Flüssigkeit,

$P_{l}\left(V_{B}\right)$ : Impuls der umströmenden Flüssigkeit.

Der Impuls der Blase wird beschrieben durch

$$
P_{B}=\rho_{B} V_{B}\left(\dot{x}_{B}+\dot{x}_{l}\right)
$$

mit

$\rho_{B}:$ Dichte des Blaseninhaltes,

$\dot{x}_{B}:$ Geschwindigkeit der Blase relativ zur Flüssigkeit.

Der Impuls der verdrängten Flüssigkeit

$$
P_{l}\left(V_{B}\right)=\frac{1}{2} \rho V_{B} \dot{x}_{B}
$$

ergibt sich aus der sogenannten virtuellen Masse, die bei sphärischen Blasen der Hälfte der durch die Blase verdrängten Masse entspricht, sowie der Relativgeschwindigkeit $\dot{x}_{B}$. Meist wird eine leere Blase angenommen $\left(\rho_{B}=0\right)$, die Bewegung wird dann durch den Impulsübertrag auf die verdrängte Flüssigkeit beschrieben $[19,16,72,91,83,87,22]$.

\footnotetext{
${ }^{1}$ In dieser Arbeit wird lediglich die eindimensionale Bewegungsgleichung verwendet.
} 
Watanabe rechnet mit volumenunabhängigem Blaseninhalt $m_{B}\left(\rho_{B}=m_{B} / V_{B}\right)$ [99]. Der mittlere Term in Gleichung (2.12) gibt den Impulsübertrag eines äquivalenten Flüssigkeitsvolumens an der Stelle der Blase wieder, dieser Term koppelt die Gleichung an die hydrodynamische Bewegungsgleichung. Im einfachen Fall einer idealen Flüssigkeit wird diese durch die Eulergleichung beschrieben. Unter Vernachlässigung des advektiven Terms reduziert diese sich auf

$$
\rho \ddot{x}_{l}=-\nabla p(x, t)=: F_{B} / V_{B} .
$$

\section{Viskose Widerstandskraft}

In einer realen Flüssigkeit muss im Allgemeinen die viskose Widerstandskraft („dragforce“) $F_{k}$ berücksichtigt werden

$$
\frac{d}{d t}\left(P_{B}+P_{l}\left(V_{B}\right)\right)=F_{B}+F_{k} .
$$

Diese Widerstandskraft ist abhängig von der Reynoldszahl ${ }^{2}$ der Translation

$$
R e=\frac{\rho R\left|\dot{x}_{B}\right|}{\eta} .
$$

In der Literatur werden folgende Ansätze verwendet:

1. Nichtviskose Flüssigkeit $[5,41]$

$$
F_{k}=0
$$

2. Stokesreibung für eine leere Blase [59]

$$
F_{k}=4 \pi \eta R \dot{x}_{B} .
$$

3. Leere Blase, $R e \ll 1[72,91]$

$$
\begin{gathered}
F_{k}=4 \pi \eta R \dot{x}_{B}+F_{H}, \\
F_{H}=8 \pi \eta \int_{0}^{t} \exp (a(s, t)) \operatorname{erfc}(a(s, t)) \frac{d}{d s}[R(s) \dot{x}] d s
\end{gathered}
$$

mit

$$
a(s, t)=\frac{9 \eta}{\rho} \int_{s}^{t} R\left(s^{\prime}\right)^{-2} d s^{\prime} .
$$

\footnotetext{
${ }^{2}$ In der Reynoldszahl, die in der Hydrodynamik das Verhältnis zwischen konvektivem $(v \cdot \nabla) v$ und diffusivem $(\eta / \rho) \Delta v$ Impulstransport beschreibt, wird gelegentlich als charakteristische Größe nicht der Radius $R$ sondern der Durchmesser $2 R$ verwendet. Dadurch ergeben sich Abweichungen in der Formulierung der Widerstandskraft.
} 
4. Stokesreibung für eine harte Kugel [19]

$$
F_{k}=6 \pi \eta R \dot{x}_{B} .
$$

5. Empirisch (Langmuir-Blodgett) [19]

$$
F_{k}=6 \pi \eta R\left(1+0.197(2 R e)^{0.63}+2.6 \cdot 10^{-4}(2 R e)^{1.38}\right) \dot{x}_{B}
$$

6. Empirisch (Haberman-Morton) [16, 99, 83]

$$
F_{k}=6.75(2 R e)^{0.22} \pi \eta R \dot{x}_{B}
$$

7. Leere Blase, $R e \gg 1[72,87,22,91]$

$$
F_{k}=12 \pi \eta R \dot{x}_{B}
$$

Magnaudet und Legendre [72] haben als zweite charakteristische Geschwindigkeit der Blasenbewegung die Radialgeschwindigkeit $\dot{R}$ eingeführt. Daraus kann eine zweite Reynoldszahl definiert werden

$$
R e_{R}=\frac{\rho R|\dot{R}|}{\eta}
$$

Für die Gültigkeit von Ausdruck (2.24) ist hinreichend, wenn $R e_{R} \gg 1$.

\section{Bewegungsgleichung einer leeren Blase im linearen Schallfeld}

In der neueren Literatur wird meist der Ansatz einer leeren Blase bei hoher Reynoldszahl im linearen Schallfeld

$$
p_{a k}(x, t)=p_{a}(x) \sin (\omega t), \quad \nabla p(x, t)=-\nabla p_{a}(x) \sin (\omega t)
$$

verwendet. Die Bewegungsgleichung lautet dann

$$
\ddot{x}_{B}(t)=-\left[\frac{18 \eta}{\rho} \frac{1}{R(t)^{2}}+\frac{3 \dot{R}(t)}{R(t)}\right] \dot{x}_{B}(t)+\frac{2 \nabla p_{a}(x)}{\rho} \sin (\omega t) .
$$




\section{Kopplung der Radialschwingung}

An die Bewegungsgleichung wird nun ein Modell für die Radialschwingungen gekoppelt

1. Linearisierte Blasengleichung [19, 16, 87],

2. RPNNP Gleichung [99],

3. Keller - Miksis Gleichung [83, 22],

4. RPNNP Modell mit kompressiblem Gas [91].

Mit Ausnahme von Doinikov [22], der der Keller-Miksis-Gleichung einen Kopplungsterm $\dot{x}_{B} / 4$ hinzufügt, werden Radial- und Translationsdynamik unidirektional gekoppelt.

\subsubsection{Die gemittelte Bewegungsgleichung}

Die über eine Schwingungsperiode $T$ der Anregung gemittelte Bewegungsgleichung (2.27) lautet

$$
\frac{\rho 2 \pi R_{T}^{3}}{3} \dot{v}_{T}=\frac{4 \pi \underline{\nabla} p_{a}}{3}\left\langle R(t)^{3} \sin (\omega t)\right\rangle_{T}-12 \pi \eta\langle R(t) \dot{x}(t)\rangle_{T}
$$

wobei die zeitliche Mittelung physikalischer Größen y gemäß

$$
\langle y\rangle_{T}=\frac{1}{T} \int_{0}^{T} y(t) d t
$$

durchzuführen ist. Ferner schreiben wir kurz $y_{T}=:\langle y\rangle_{T}$. Die Mittelung der Widerstandskraft ist problematisch, da über das Produkt von Geschwindigkeit und Radius gemittelt werden muss. Diesem Problem ist in der Arbeit ein eigenes Kapitel gewidmet (siehe Kapitel 3). Der erste Term auf der rechten Seite beschreibt die gemittelte Kraft des Schallfeldes auf die Blase. Diese Nettokraft wird auch primäre Bjerkneskraft genannt ${ }^{3}$.

\footnotetext{
${ }^{3}$ Gelegentlich wird der Begriff auch für die zeitaufgelöste Kraft des Schalldruckgradienten auf die pulsierende Blase verwendet [91].
} 


\subsubsection{Primäre Bjerkneskraft}

Die primäre Bjerkneskraft

$$
F_{b j 1}=\frac{4 \pi \underline{\nabla} p_{a}}{3}\left\langle R(t)^{3} \sin (\omega t)\right\rangle_{T}
$$

lässt sich bei bekannter Radialschwingung numerisch lösen. Bei harmonischer Blasenschwingung und linearem Schallfeld kann die primäre Bjerkneskraft analytisch gelöst werden.

\section{Lineare Blasenschwingung im Stehwellenfeld}

Die Schalldruckverteilung einer akustischen Stehwelle im Quader kann in einer Raumrichtung x durch

$$
\begin{array}{r}
p_{a k}(x, t)=p_{a} \cos \left(k_{x} x\right) \sin (\omega t) \\
\nabla p_{a k}(x, t)=-k_{x} p_{a} \sin \left(k_{x} x\right) \sin (\omega t),
\end{array}
$$

mit

$k_{x}:$ Wellenzahl

beschrieben werden. Der Ursprung des Koordinatensystems wird dabei in den Schalldruckbauch gelegt. Die Pulsation der Blase setzen wir nach Gleichung (2.7) linearisiert an und können die Auslenkung, die direkt proportional zur Schalldruckamplitude ist, durch die Auslenkung im Druckmaximum $R_{a, 0}$ und den Ort beschreiben

$$
R_{a}=R_{a, 0} \cos \left(k_{x} x\right) .
$$

Die primäre Bjerkneskraft ergibt sich unter Vernachlässigung von Termen höherer Ordnung zu

$$
F_{b j 1}=-\pi k_{x} p_{a} R_{0}^{2} R_{a, 0} \cos (\phi) \sin \left(2 k_{x} x\right) .
$$

Die Kraft verschwindet, wenn die Blase in Resonanz schwingt $(\phi=-\pi / 2)$. Für Blasen, die unterhalb ihrer Resonanzfrequenz angetrieben werden (subresonant), ist der Schalldruckbauch attraktiv, für superresonant schwingende Blasen wird er repulsiv. Die primäre Bjerkneskraft wird maximal an der Stelle

$$
x\left(F_{b j 1, \max }\right)=\frac{\pi}{4 k_{x}},
$$


also gerade auf halber Strecke zwischen Schalldruckbauch und -knoten. Das zeitliche Mittel des Produktes einer sinusförmigen Schwingung mit einer höheren Harmonischen verschwindet unabhängig von der Phasenverschiebung. Damit haben sowohl höhere Harmonische in der Blasenschwingung bei harmonischer Anregung wie auch höhere Harmonische in der Anregung bei linearer Blasenschwingung keinen Einfluss auf die Bjerkneskraft. Erst wenn sowohl Schallfeld als auch Blase nichtlinear schwingen, haben die höheren Harmonischen einen Effekt.

\section{Lineare Blasenschwingung in laufender ebener Welle}

In einer ebenen, harmonischen Schallwelle, die sich in x-Richtung ausbreitet, gilt für Schalldruckverteilung und Druckgradient

$$
\begin{aligned}
p_{a k}(x, t) & =p_{a} \sin \left(\omega t-k_{x} x\right) \\
\nabla p_{a k}(x, t) & =-k_{x} p_{a} \cos \left(\omega t-k_{x} x\right) .
\end{aligned}
$$

Die lineare Blasenschwingung wird hier beschrieben durch

$$
R(t)=R_{0}-R_{a} \sin \left(\omega t-k_{x} x+\phi\right),
$$

die primäre Bjerkneskraft ergibt sich zu

$$
F_{b j 1}=-2 \pi k_{x} p_{a} R_{0}^{2} R_{a} \sin (\phi)
$$

Hier wird die Kraft für resonant schwingende Blasen maximal. Die primäre Bjerkneskraft wirkt dann in Richtung der Wellenausbreitung.

\subsubsection{Aufsteigende Blasen}

Durch die Gravitation der Erde wirkt auf eine Blase in z - Richtung durch den Schweredruck der Flüssigkeit zusätzlich die Auftriebskraft

$$
F_{h s}=V_{B}\left(\rho-\rho_{B}\right) g,
$$

mit

$g$ : Schwerebeschleunigung.

Diese führt dazu, dass Blasen in der Flüssigkeit aufsteigen. 


\subsection{Diffusionsprozesse}

Die Phasengrenze zwischen Flüssigkeit und Hohlraum ist durchlässig für Dampf und Gas. Bei der Betrachtung der Radialdynamik (siehe Abschnitt 2.1) haben wir eine konstante Gasmenge angenommen, während wir den Dampfgehalt durch den Dampfdruck bestimmt haben. Die Annahme, dass während einer Oszillation Kondensation und Verdampfung stattfindet, jedoch keine Gasdiffusion, ist durch die Annahme gerechtfertigt, dass Diffusion auf einer sehr viel längeren Zeitskala stattfindet. Für das Langzeitverhalten von Blasen im Schallfeld muss Diffusion jedoch berücksichtigt werden [17].

\section{Partialdruck gelöster Gase}

Für in geringer Konzentration gelöste Gase wird im Gleichgewicht der Partialdruck in der Gasphase durch das Henry'sche Gesetz bestimmt

$$
c_{x}=h_{x} p_{R, x}^{*}
$$

mit

$c_{x}$ : Konzentration des Gases $\mathrm{x}$ an der Phasengrenze,

$h_{x}$ : Henrykonstante des Gases $\mathrm{x}$ in der Flüssigkeit (temperaturabhängig),

$p_{R, x}^{*}$ : Gleichgewichts-Partialdruck des Gases $\mathrm{x}$ in der Gasphase.

\section{Diffusionsrate einer nichtschwingenden Blase}

In gesättigter Lösung in Abwesenheit von anderen Gasen und unter Vernachlässigung des Dampfdrucks entspricht der Partialdruck gerade dem hydrostatischen Druck

$$
c_{x, 0}=h_{x} p_{h s} .
$$

Die Gaskonzentration an der Wand einer Gasblase im Gleichgewicht ist immer höher als die Sättigungskonzentration, da der Druck in der Blase die Oberflächenspannung ausgleichen muss

$$
\begin{aligned}
& p_{x, R_{0}}=p_{h s}+\frac{2 \sigma}{R_{0}} \\
& c_{x, R_{0}}=c_{x, 0}+\frac{2 \sigma}{R_{0} h_{x}} .
\end{aligned}
$$


Gasdiffusion findet dann nach dem zweiten Fickschen Gesetz

$$
\frac{\partial c_{x}}{\partial t}+\dot{r} \frac{\partial c_{x}}{\partial r}=\frac{D_{x}}{r^{2}} \frac{\partial}{\partial r}\left(r^{2} \frac{\partial c_{x}}{\partial r}\right)
$$

von der Blase weg ins Fernfeld statt. Der Materiefluss an der Phasengrenze wird durch das erste Ficksche Gesetz beschrieben

$$
\frac{\mathrm{d} m_{x}}{\mathrm{~d} t}=-\left.4 \pi R^{2} D_{x} \frac{\partial c_{x}}{\partial r}\right|_{R}
$$

mit

$m_{x}:$ Masse des Gases $\mathrm{x}$,

$D_{x}$ : Diffusionskoeffizient des Gases x.

Epstein und Plesset haben 1950 die Diffusionsrate solcher Blasen bestimmt [27]

$$
\begin{aligned}
R \dot{R} & =\frac{D_{x}\left[c_{x, \infty}-c_{x, R}\left(1+2 \sigma / R p_{h s}\right)\right]}{\rho_{R}\left(1+4 \sigma /\left(3 R p_{h s}\right)\right.}\left[1+\frac{R}{\sqrt{\pi D t}}\right] \\
R_{0}^{2}(t)-R_{0}^{2}(0) & \approx\left(\frac{2 D_{x}\left(c_{x, \infty}-c_{x, R}\right)}{\rho_{R}}\right) t
\end{aligned}
$$

mit

$\rho_{R}:$ Dichte des Gases in der Blase,

$c_{x, \infty}$ : Gaskonzentration im Fernfeld der Blase.

Zur völligen Auflösung benötigt eine Blase damit

$$
t_{\text {dissolve }} \approx \frac{\rho_{R} R_{0}^{2}}{2 D_{x}\left(c_{x, \infty}-c_{x, R}\right)} .
$$

\section{Diffusion einer pulsierenden Blase}

Durch die Druckänderung in der Blase während der Volumenoszillation ändert sich beständig das Konzentrationsgefälle an der Blasenwand. Um Diffusion und Radialdynamik zu entkoppeln, gibt man die Bewegung der Blasenwand vor und ermittelt den mittleren Materiefluss über eine Schwingungsperiode. 


\section{Lineare Blasenschwingung}

Für die linearisierte Blasenschwingung (Gleichung (3.3) in Abschnitt 3) in gesättigter Lösung bestimmten Hsieh und Plesset 1961 die Wachstumsrate des Ruheradius einer pulsierenden Blase [48]

$$
\frac{\mathrm{d} R_{0}}{\mathrm{~d} t}=\frac{2 D_{x} c_{x, 0} \epsilon^{2}}{3 \rho_{R} R_{0}}
$$

\section{Nichtlineare Blasenschwingung}

Eller und Flynn haben für beliebige nichtlineare periodische Blasenschwingungen und beliebige Gaskonzentrationen $c_{x, \infty}$ folgenden Ausdruck für den Gleichgewichtsradius gefunden [25]

$$
\frac{c_{x, \infty}}{c_{x, 0}}=1+\frac{2 \sigma}{R_{0, c_{x}} p_{h s}} \frac{\left\langle R(t) / R_{0}\right\rangle_{T}}{\left\langle\left(R(t) / R_{0}\right)^{4}\right\rangle_{T}} .
$$

Fyrillas und Szeri entwickelten dann ein Modell, das auch fern vom Diffusionsgleichgewicht den Nettomassentransport wiedergibt [31]

$$
\frac{\mathrm{d} m_{x, R_{0}}}{\mathrm{~d} \tau}=\frac{c_{x, \infty} / c_{x, 0}-\left\langle c_{x} / c_{x, 0}\right\rangle_{\tau}}{T_{r d}}
$$

mit

$$
\begin{aligned}
& m_{x, R_{0}} \text { : Gasmenge relativ zur verdrängten Gasmenge bei } R_{0}, \\
& \tau=t D_{x} / R_{0}^{2} \text { : Normierte Zeit, } \\
& c_{x, \infty}: \text { Gleichgewichtskonzentration fern der Blase, } \\
& c_{x, 0}=h_{x} p_{h s}: \text { Sättigungskonzentration, } \\
& T_{r d}=\int_{0}^{\infty} \mathrm{d} \xi\left(1 / T \int_{0}^{T}\left(3 \xi+\left(R / R_{0}\right)^{3}\right)^{\frac{3}{4}} \mathrm{~d} t\right)^{-1}, \\
& \xi=\left(r^{3}-R^{3}\right) /\left(3 R_{0}^{3}\right) \\
& <\cdot>_{\tau}=\left(\int_{0}^{T} R^{4}(t) \mathrm{d} t\right)^{-1} \int_{0}^{T} R^{4}(t) \cdot \mathrm{d} t
\end{aligned}
$$

$T_{r d}$ ist immer positiv. Deshalb wird die Richtung des Materieflusses durch das Verhältnis von Gleichgewichtskonzentration und nichtlinear gemittelter Gaskonzentration an der Blasenwand bestimmt. Lösungen können nun numerisch erhalten werden. Für niedrige Schalldruckamplituden und hohe Gaskonzentrationen können nur instabile Gleichgewichtskonzentrationen gefunden werden, während sich bei geringen Gaskonzentrationen und hohem Schalldruck auch diffusionsstabile Gleichgewichtsradien ergeben. Diese wachsen mit zunehmendem Gasgehalt und Schalldruck. 


\subsection{Oberflächeninstabilitäten}

Störungen der Sphärizität einer Blase können durch Anregung von Oberflächenmoden verursacht werden, die sich ähnlich einer Rayleigh-Taylor-Instabilität während der Volumenoszillation parametrisch verstärken können.

Bei periodischen Oberflächenschwingungen kann die Blasenwand nach den $\mathrm{Ku}$ gelflächenfunktionen entwickelt werden [85, 30]:

$$
\tilde{R}(\phi, \theta, t)=R(t)+\sum_{l=1}^{\infty} \sum_{\varpi=-l}^{+l} a_{l, \varpi}(t) Y_{l, \varpi}(\phi, \theta),
$$

mit

$$
\begin{aligned}
& Y_{l, \varpi}(\phi, \theta)=\sqrt{\frac{(2 l+1)(l-\varpi) !}{4 \pi(l+\varpi) !}} P_{l}^{\varpi}(\cos \theta) e^{i \varpi \phi}: \text { Kugelflächenfunktion, } \\
& P_{l}^{\varpi}(x)=\frac{(-1)^{\varpi}}{2^{l} l !}\left(1-x^{2}\right)^{\frac{\varpi}{2}} \frac{d^{l+\varpi}}{d x^{l+\varpi}}\left(x^{2}-1\right)^{l}: \text { Legendre-Polynome. }
\end{aligned}
$$

Bei kleinen Auslenkungen $a_{l, \varpi}(t) \ll R(t)$ sind die einzelnen Moden unabhängig voneinander. Unter der Voraussetzung der Periodizität in $R(t)$ ergeben sich für

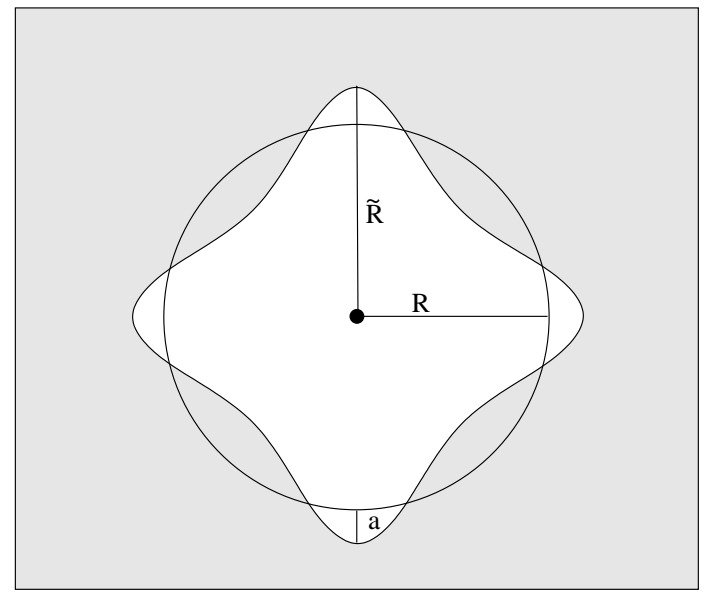

Abbildung 2.1: Der Entwicklungskoeffizient $a_{l, \varpi}$, hier für $l=4$, beschreibt die Abweichung von der Kugelsymmetrie. Er ist in $\varpi$ entartet, $\varpi$ ist aber wegen der Projektion auf die x-y-Ebene nicht darstellbar.

den Entwicklungskoeffizienten $a_{l, \varpi}$ harmonische, parametrisch angeregte Schwingungen

$$
\ddot{a}_{l, \varpi}+B(R(t)) \dot{a}_{l, \varpi}-A(R(t)) a_{l, \varpi}=0 .
$$

Nach Lamb gilt für die Resonanzradien der Oberflächenmoden einer Luftblase $[58]$

$$
R_{n}^{3}=(l+1)(l-1)(l+2) \sigma /\left(\rho \omega^{2}\right) .
$$




\section{Bewegungsgleichung der Entwicklungskoeffizienten}

Plesset bestimmt 1954 die Koeffizienten $A$ und $B$ für die Oberflächenschwingung eines Flüssigkeitstropfens in einer idealen Flüssigkeit [85]. Die leere Blase ist dabei ein Spezialfall [86]

$$
\begin{aligned}
B(R(t))_{l, \text { varpi }} & =\frac{3 \dot{R}}{R} \\
A(R(t))_{l, \varpi} & =(l-1)\left[\frac{\ddot{R}}{R}-\frac{(l+2)(l+1) \sigma}{\rho_{0} R^{3}}\right] .
\end{aligned}
$$

Prosperetti erweitert 1977 den Ansatz für eine viskose Flüssigkeit [90]. Vernachlässigt man die in der Flüssigkeit entstehenden Wirbel, so ergibt sich für die Koeffizienten

$$
\begin{aligned}
B(R(t))_{l, \varpi} & =\frac{3 \dot{R}}{R}+(l+2)(2 l+1) \frac{2 \mu}{\rho_{0} R^{2}} \\
A(R(t))_{l, \varpi} & =(l-1)\left[\frac{\ddot{R}}{R}-\frac{(l+2)}{\rho_{0} R^{3}}((l+1) \sigma+2 \mu \dot{R})\right] .
\end{aligned}
$$

\section{Stabilitätsbetrachtung}

Zur Betrachtung der Oberflächenstabilität der Blasen können zwei Kriterien herangezogen werden:

1. Parametrische Instabilität: Wenn nach dem Einschwingvorgang $R(t)$ periodisch mit der Anregungsfrequenz oszilliert, so gilt dies auch für die Koeffizienten von $a_{l, \varpi}$ und $\dot{a}_{l, \varpi}$ in obigen Differentialgleichungen $(A$ bzw. $B)$. Dann ist die Abbildung

$$
\left(\begin{array}{c}
a_{l, \varpi}(0) \\
\dot{a}_{l, \varpi}(0)
\end{array}\right) \rightarrow\left(\begin{array}{c}
a_{l, \varpi}(T) \\
\dot{a}_{l, \varpi}(T)
\end{array}\right)
$$

linear und die Floquetmatrix $F_{l}(T)$

$$
\left(\begin{array}{c}
a_{l, \varpi}(T) \\
\dot{a}_{l, \varpi}(T)
\end{array}\right)=F_{l}(T)\left(\begin{array}{c}
a_{l, \varpi}(0) \\
\dot{a}_{l, \varpi}(0)
\end{array}\right)
$$

beschreibt die Entwicklung der Anfangsbedingungen über eine Schwingungsperiode. Ist der Betrag des betragsgrößten Eigenwertes der Floquetmatrix $\left|\lambda_{\text {max }}\right|$ größer als 1, so verstärkt sich die Oberflächenschwingung mit jeder Periode und wird so nach einer Weile die Zerstörung der Blase verursachen. Die Floquetmatrix kann numerisch berechnet werden. 
2. Verstärkung einer Störung: Während die parametrische Instabilität das Aufschaukeln einer angeregten Mode über viele Schwingungsperioden beschreibt, kann natürlich auch kurzzeitig starkes Aufschwingen der Oberflächenschwingung die Blase zerstören. Insbesondere in der Nähe des Minimalradius der Blase, wenn $\ddot{R}>0$, wächst eine nichtsphärische Störung stark an. Als Kriterium kann das Verhältnis

$$
\frac{a_{l, \varpi}(t)}{R(t)}
$$

betrachtet werden. Ist dieses größer als 1, so ist die Blase mit Sicherheit nicht mehr stabil.

Zur Berechnung wird der zeitliche Verlauf des Entwicklungskoeffizienten unter Anwendung kleiner Rauschterme betrachtet. Numerische Simulationen zeigen, dass die $l=2$ Mode schneller instabil wird als höhere Oberflächenmoden [74]. Außerdem ist parametrische Instabilität in weiten Parameterbereichen das striktere Kriterium.

\subsection{Wechselwirkung benachbarter Blasen}

\subsubsection{Radialdynamik}

Eine pulsierende Blase emittiert ihrerseits ein Schallfeld, welches das Schwingungsverhalten benachbarter Blasen beeinflussen kann. Betrachten wir als ersten Ansatz eine inkompressible Strömung, so ist die Geschwindigkeit an jedem Punkt der Flüssigkeit durch die Bewegung der Wand der Blase $i$ gemäß

$$
\dot{r}=\frac{R_{i}^{2} \dot{R}_{i}}{r^{2}}
$$

gegeben. Dabei bezeichnet

$r$ : Abstand vom Blasenmittelpunkt,

$\dot{r}$ : Geschwindigkeit der Flüssigkeit in Kugelkoordinaten.

Daraus folgt mit Gleichung (2.15) für den durch die Blase verursachten Druckgradienten $\nabla_{r} p_{i}$ sowie den Druck $p_{i}$

$$
\begin{aligned}
\nabla_{r} p_{i} & =-\frac{\rho}{r^{2}} \frac{\mathrm{d}}{\mathrm{d} t}\left(R_{i}^{2} \dot{R}_{i}\right) \\
p_{i} & =\frac{\rho}{r} \frac{\mathrm{d}}{\mathrm{d} t}\left(R_{i}^{2} \dot{R}_{i}\right) .
\end{aligned}
$$


Am Ort der Blase $j$, die sich in einer Entfernung $d$ von Blase $i$ befindet, muss also der Druck in der Flüssigkeit um $p_{i}$ ergänzt werden. Das bedeutet, dass in der Rayleigh-Plesset Gleichung (2.1)

$$
p_{\infty, i}=p_{\infty}+p_{i}
$$

bzw. in der RPNNP (2.2) sowie der Keller-Miksis Gleichung (2.3)

$$
p_{l, i}=p_{l}-p_{i}
$$

$\mathrm{zu}$ verwenden sind.

Ebenso wirkt Blase $j$ auf Blase $i$, deshalb muss die gekoppelte Radialdynamik der beiden Blasen simultan gelöst werden. In realen Blasenfeldern ist die Näherung der Inkompressibilität nicht gegeben. Es müssen Schalllaufzeiten berücksichtigt werden, die durch die Einführung eines Zeitdelays implementiert werden können [75]. Die bekannte Emission von Stoßwellen in Kavitationsfeldern ist hingegen schwer zu modellieren.

\subsubsection{Translationsdynamik}

Die durch die Flüssigkeitsbewegung induzierte Kraft wird wieder durch Gleichung (2.30) beschrieben. Nehmen wir eine ruhende Kugel an und ersetzen dort $\ddot{x}_{l}$ wieder mit Gleichung (2.15), so erhalten wir für die Kraft $F_{B, i j}$ der Blase $i$ auf die Blase $j$ in Richtung des Mittelpunktes von Blase $i$ in der Entfernung $d$

$$
F_{B, i, j}=\frac{\rho}{4 \pi d^{2}} V_{j} \ddot{V}_{i}
$$

Ist $F_{B, i, j}$ positiv, so ziehen sich die Blasen an, bei negativem Vorzeichen stoßen sie sich ab. Bjerknes beschrieb auch für den Fall zweier pulsierender Kugeln die Nettokraft [9]. Deshalb wird die über eine Schwingungsperiode gemittelte Kraft

$$
\left\langle F_{B, i, j}\right\rangle_{T}=\frac{\rho}{4 \pi d^{2}}\left\langle V_{j} \ddot{V}_{i}\right\rangle_{T}
$$

auch zweite oder sekundäre Bjerkneskraft genannt [16]. Wird die endliche Schalllaufzeit wieder vernachlässigt, so sind die sekundären Bjerkneskräfte auf die beiden Blasen symmetrisch und es ergibt sich durch partielle Integration

$$
F_{B, i, j}=F_{B, j, i}=F_{b j 2}=\frac{\rho}{4 \pi d^{2}}\left\langle\dot{V}_{j} \dot{V}_{i}\right\rangle_{T} .
$$




\section{Kapitel 3}

\section{Die Mittelung der linearisierten Bewegungsgleichung}

\section{Mittelung des Reibungskraft}

Bei Untersuchungen der Blasenbewegung auf großen Zeitskalen wird oft die über eine Anregungsperiode gemittelte Bewegungsgleichung verwendet (siehe Abschnitt 2.2.2). In der gemittelten Bewegungsgleichung für die Translation Gl. (2.28) wird im Term der viskosen Widerstandskraft

$$
\left\langle F_{k}\right\rangle_{T}=-12 \pi \eta\langle R(t) \dot{x}(t)\rangle_{T}
$$

der Mittelwert über das Produkt von Radialschwingung und Translationsgeschwindigkeit gebildet. Um eine Bewegungsgleichung für die mittlere Geschwindigkeit aufstellen zu können, müssten Radius und Geschwindigkeit getrennt voneinander gemittelt werden. Dies ist unter den folgenden Annahmen möglich:

1. Kleiner oszillatorischer Anteil $\tilde{R}$ in der Radialbewegung:

$$
R(t)=R_{T}+\tilde{R}, \quad \tilde{R} \ll R_{T},
$$

2. Kleiner oszillatorischer Anteil $\tilde{v}$ in der Translationsdynamik:

$$
\dot{x}=v_{T}+\tilde{v}, \quad \tilde{v} \ll v_{T} .
$$

Das zeitliche Mittel von Radius und Geschwindigkeit kann dann als

$$
\langle R(t) \dot{x}(t)\rangle_{T}=R_{T} v_{T}+\langle\tilde{R}\rangle_{T} v_{T}+\langle\tilde{v}\rangle_{T} R_{T}+\langle\tilde{R} \tilde{v}\rangle_{T}
$$

formuliert werden. 
Da das zeitliche Mittel der oszillatorischen Anteile per Definition verschwindet, fallen die beiden mittleren Terme der rechten Seite weg. Ferner ist nach Voraussetzung $\langle\tilde{R} \tilde{v}\rangle_{T} \ll R_{T} v_{T}$. Vernachlässigen wir $\langle\tilde{R} \tilde{v}\rangle_{T}$, dann können wir den Mittelwert des Produktes von Radius und Geschwindigkeit durch das Produkt der Mittelwerte ersetzen

$$
\langle R(t) \dot{x}(t)\rangle_{T} \approx R_{T} v_{T}
$$

Bei stark nichtlinearer Radialschwingung ist Voraussetzung (1) nicht erfüllt, da die Auslenkung um bis zu einer Größenordnung über dem Ruheradius liegen kann. Voraussetzung (2) gilt ebenfalls nicht zwangsläufig, da das Verhältnis von mittlerer Geschwindigkeit und oszillatorischem Anteil stark von der Form der Radialschwingung abhängt. Für eine linear resonant schwingende Blase verschwindet im akustischen Stehwellenfeld die Nettokraft, sie oszilliert in der Anregungsfrequenz um ihre Ruhelage (siehe Abschnitt 2.2.3). Dann gilt Voraussetzung (2) nicht. Wird die Blasenbewegung durch eine gerichtete Kraft wie zum Beispiel die Auftriebskraft dominiert, so wird man den oszillatorischen Anteil der zusätzlichen periodischen Kraft vernachlässigen können. Reddy und Szeri [91] haben numerisch die mittlere Geschwindigkeit sowohl aus der zeitaufgelösten als auch aus der gemittelten Bewegungsgleichung berechnet und einen relativen Fehler von bis zu $120 \%$ erhalten. Für Blasen im schwachen Schallfeld haben sie geringe Unterschiede festgestellt und daher die Näherung (3.2) als anwendbar bewertet. Dass dies selbst bei linearisierter Blasenschwingung im schwachen Schallfeld zu Fehlern bis zu 30\% führen kann, wird im folgenden gezeigt.

\section{Die Lösung der zeitaufgelösten Bewegungsgleichung}

Wir betrachten den einfachen Fall einer eindimensionalen Bewegung einer leeren Blase in einem akustischen Stehwellenfeld (Gl. (2.27)). Die Radialdynamik wird als harmonisch angenommen (siehe Gl. (2.7))

$$
R(t)=R_{0}[1-\epsilon \sin (\omega t)] .
$$

Diese Phasenlage gilt im Fall subresonant schwingender Blasen, das heißt für

$$
\omega<\omega_{0}
$$

Wir linearisieren die radiusabhängigen Terme in der Bewegungsgleichung

$$
\begin{aligned}
\frac{1}{[1-\epsilon \sin (\omega t)]^{2}} & \approx 1+2 \epsilon \sin (\omega t) \\
\frac{\dot{R}(t)}{R(t)} & \approx-\epsilon \omega \cos (\omega t) .
\end{aligned}
$$


Die Translationsdynamik kann dann durch

$$
\begin{aligned}
\ddot{x} & =-\left[\frac{18 \eta}{2 \rho R_{0}^{2}}[1+2 \epsilon \sin (\omega t)]-3 \epsilon \omega \cos (\omega t)\right] \dot{x}+\frac{2 \nabla p_{a}}{\rho} \sin (\omega t) \\
\Leftrightarrow \quad \ddot{x} & =-\left[k_{1}+2 k_{1} \epsilon \sin (\omega t)-3 \epsilon \omega \cos (\omega t)\right] \dot{x}+k_{2} \sin (\omega t) \\
\Leftrightarrow \quad \ddot{x} & =-\left[k_{1}+\epsilon k_{3} \sin \left(\omega t+k_{4}\right)\right] \dot{x}+k_{2} \sin (\omega t),
\end{aligned}
$$

mit

$$
\begin{aligned}
k_{1} & =\frac{18 \eta}{2 \rho r_{0}^{2}}, \\
k_{2} & =\frac{2 \nabla p_{a}}{\rho}, \\
k_{3} & =\sqrt{\left(2 k_{1}\right)^{2}+(3 \omega)^{2}}, \\
k_{4} & =\arctan \left(\frac{2 k_{x} p_{a}}{\rho}\right)
\end{aligned}
$$

beschrieben werden. Dies ist eine gewöhnliche Differentialgleichung zweiter Ordnung, die mit Standardmethoden (Variation der Konstanten) gelöst werden kann [29]. Wir erhalten für die Geschwindigkeit

$$
\begin{aligned}
\dot{x}(t)=- & \epsilon\left[k_{10}+k_{5} k_{8} \cos ^{2}(\omega t)\right. \\
& \left.+\left(k_{1} 1+k_{6} k_{7}\right) \sin ^{2}(\omega t)\right] \\
+ & k_{7} \sin (\omega t)+k_{8} \cos (\omega t) \\
+ & \epsilon\left(k_{5} k_{7}+k_{6} k_{8}-k_{9}\right) \cos (\omega t) \sin (\omega t) \\
+ & \exp \left(-k_{1} t\right)\left[\dot{x}_{0}-k_{8}-\epsilon k_{10}\right. \\
& +\epsilon k_{5}\left(\dot{x}_{0}-k_{8}\right) \cos (\omega t) \\
& \left.+\epsilon k_{6}\left(\dot{x}_{0}-k_{8}\right) \sin (\omega t)\right] \\
+ & \mathcal{O}\left(\epsilon^{2}\right),
\end{aligned}
$$

mit

$$
\begin{aligned}
k_{5} & =k_{3} \cos \left(k_{4}\right) / \omega \\
k_{6} & =k_{3} \sin \left(k_{4}\right) / \omega \\
k_{7} & =k_{2} \cos (\arctan (-c))\left(k_{1}^{2}+\omega^{2}\right)^{-1 / 2} \\
k_{8} & =k_{2} \sin (\arctan (-c))\left(k_{1}^{2}+\omega^{2}\right)^{-1 / 2}, \\
k_{9} & =k_{2}\left(k_{5}+2 c k_{6}\right)\left(k_{1}\left(1+(2 c)^{2}\right)\right)^{-1}, \\
k_{10} & =k_{9} c \\
k_{11} & =2 k_{10}-k_{2} k_{6} / k_{1} .
\end{aligned}
$$


Dabei haben wir den Parameter

$$
c=\frac{\omega}{k_{1}}
$$

eingeführt. Wir werden feststellen, dass die mittlere Geschwindigkeit durch $c$ parametrisiert werden kann. Da wir eine quasistationäre Lösung suchen, können wir Terme in der Translationsgeschwindigkeit, die mit $\exp \left(-k_{1} t\right)$ abfallen, vernachlässigen. Der oszillatorische Teil der Geschwindigkeit setzt sich aus Termen zusammen, die proportional zu $\sin (\omega t), \cos (\omega t)$ und $\cos (\omega t) \sin (\omega t)$ sind. Diese Terme verschwinden im zeitlichen Mittel. Zur Nettogeschwindigkeit tragen lediglich die Terme bei, die proportional zu $\sin ^{2}(\omega t)$ bzw. $\cos ^{2}(\omega t)$ sind. Insgesamt erhalten wir als mittlere Geschwindigkeit im quasistationären Fall

$$
\begin{aligned}
v_{T}= & \frac{\epsilon \sqrt{\left.\left(2 k_{1}\right)^{2}+(3 \omega)^{2}\right)} k_{2}}{2 \omega} \\
& \times\left[\frac{1}{\sqrt{k_{1}^{2}+\omega^{2}}} \sin \left(\arctan \left(\frac{\omega k_{1}}{2 k_{1}^{2}+3 \omega^{2}}\right)\right)\right. \\
& \left.+\frac{\sin \left(\arctan \left(\frac{-3 \omega}{2 k_{1}}\right)\right)}{k_{1}}\right] \\
= & \left.\frac{3 \epsilon k_{2}}{2 k_{1}} \sqrt{\left(\frac{2}{3 c}\right)^{2}+1}\right] \\
& \times\left[\frac{1}{\sqrt{1+c^{2}}} \sin \left(\arctan \left(\frac{c}{2+3 c^{2}}\right)\right)\right. \\
& \left.+\sin \left(\arctan \left(\frac{-3 c}{2}\right)\right)\right] \\
= & -\frac{3 \epsilon k_{2}}{2 k_{1}} f(c) .
\end{aligned}
$$

Die mittlere Geschwindigkeit kann also durch eine von $c$ abhängige Funktion und einen Vorfaktor $\left(-3 \epsilon k_{2}\right) /\left(2 k_{1}\right)$ beschrieben werden. Der Verlauf von $f(c)$ ist in Abbildung 3.1 dargestellt. Die Funktion nimmt Werte im (einseitig offenen) Intervall $] 2 / 3,1]$ an. Das bedeutet, dass die mittlere Geschwindigkeit im wesentlichen durch den Vorfaktor bestimmt ist. Sie muss aber für kleine Werte von $c$ etwas nach unten korrigiert werden. 


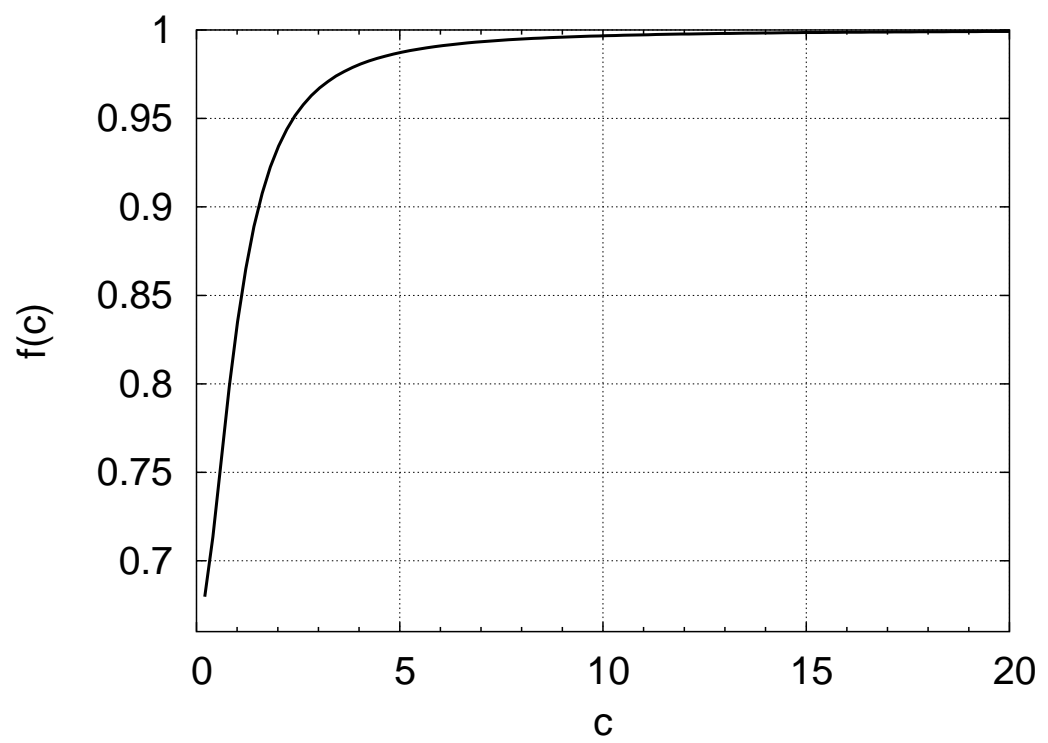

Abbildung 3.1: Die Korrekturfunktion f(c) der Translationsgeschwindigkeit.

\section{Die Lösung der gemittelten Bewegungsgleichung}

Mitteln wir Bewegungsgleichung (3.5) unter Benutzung der Näherung (3.2), so erhalten wir für die mittlere Geschwindigkeit $v_{T, a}$ die Bewegungsgleichung

$$
\begin{array}{r}
\frac{2 \pi \rho}{3} R_{0}^{3} \dot{v}_{T, a}+=-12 \pi \eta R_{0} v_{T, a}-\nabla p_{a} \frac{4 \pi}{3} R_{0}^{3} \frac{3}{2} \epsilon \\
\dot{v}_{T, a}=-\frac{18 \eta}{2 \rho R_{0}^{2}} v_{T, a}-\frac{3 \epsilon}{2} \frac{2 \nabla p_{a}}{\rho} \\
\dot{v}_{T, a}=-k_{1} v_{T, a}-\frac{3 \epsilon}{2} k_{2} .
\end{array}
$$

Dies ist eine gewöhnliche Differentialgleichung erster Ordnung für die mittlere Geschwindigkeit. Im quasistationären Fall erhalten wir

$$
\bar{v}_{T, a}=-\frac{3 \epsilon k_{2}}{2 k_{1}} .
$$

Dies ist gerade der Vorfaktor der zeitaufgelösten Lösung.

\section{Vergleich der Lösungen}

Für große Werte von $c$ gehen die beiden Lösungen ineinander über, für kleine Werte von $c$ wird die Geschwindigkeit durch die gemittelte Bewegungsgleichung 
überschätzt. $c$ ist durch die äußeren Parameter gemäß

$$
c=\frac{\omega}{k_{1}}=\frac{\omega \rho R_{0}^{2}}{18 \eta}
$$

bestimmt. Hohe Werte von $c$ werden in Flüssigkeiten mit hoher Dichte und geringer Zähigkeit erreicht. Speziell für Wasser mit einer Dichte von $\rho_{w}=10^{3} \frac{\mathrm{kg}}{\mathrm{m}^{3}}$ und einer dynamischen Zähigkeit von $\eta_{w}=10^{-3} \frac{\mathrm{kg}}{\mathrm{ms}}$ gilt

$$
c_{w}=\frac{10^{6}}{18} \omega R_{0}^{2}
$$

bzw. unter Anwendung der Minnaert-Frequenz (siehe Kapitel 2, Gl.(2.10)).

$$
c_{w}=\frac{10^{6} \pi}{3} \frac{R_{0}^{2}}{R_{r}} .
$$

In Abbildung 3.2 ist der Fehler der aus der gemittelten Bewegungsgleichung ermittelten Geschwindigkeit gegen das Produkt aus Ruheradius und Verhältnis zwischen Ruheradius und Resonanzradius aufgetragen. Zur Veranschaulichung

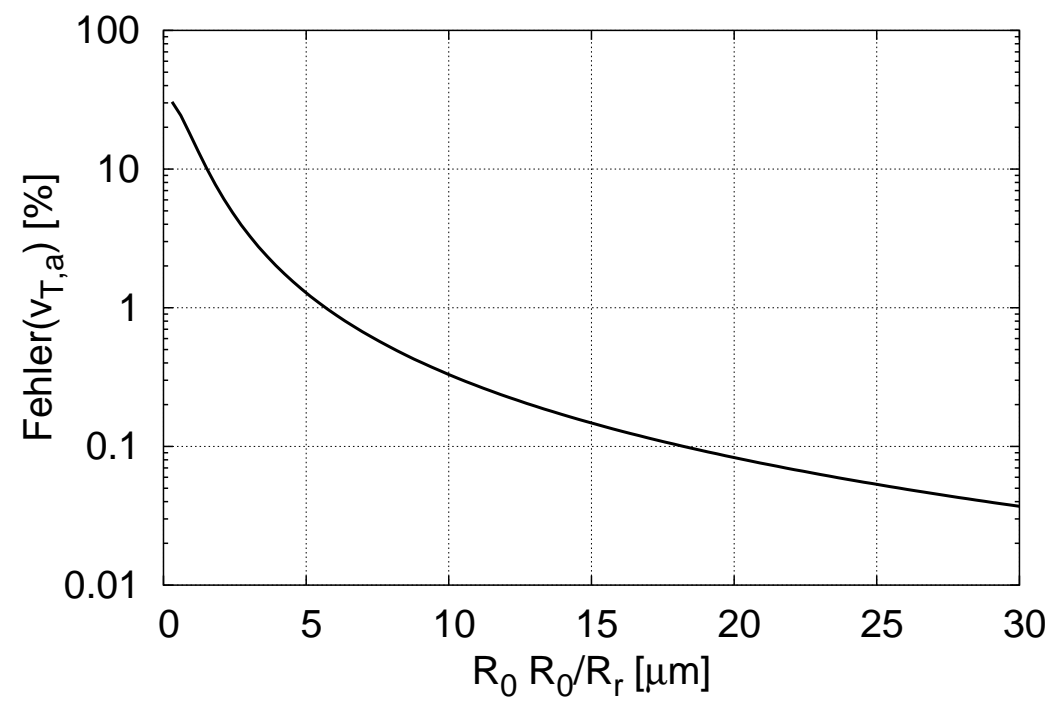

Abbildung 3.2: Fehler von $v_{T, a}$ in Abhängigkeit von Ruheradius und Resonanzradius.

sind in Abbildung 3.3 der Ruheradius sowie die Grenzradien für einen Fehler von $1 \%$ bzw. $0.1 \%$ für den nahen Ultraschallbereich aufgetragen. Gerade im unteren Frequenzbereich gibt es einen nennenswerten Radiusbereich, in dem die gemittelte Bewegungsgleichung eine gute Näherung ist. Bei kleineren Blasen ist es jedoch ratsam, die Korrekturfunktion $f(c)$ zu verwenden, wenn die gemittelte Bewegungsgleichung verwendet werden soll. 


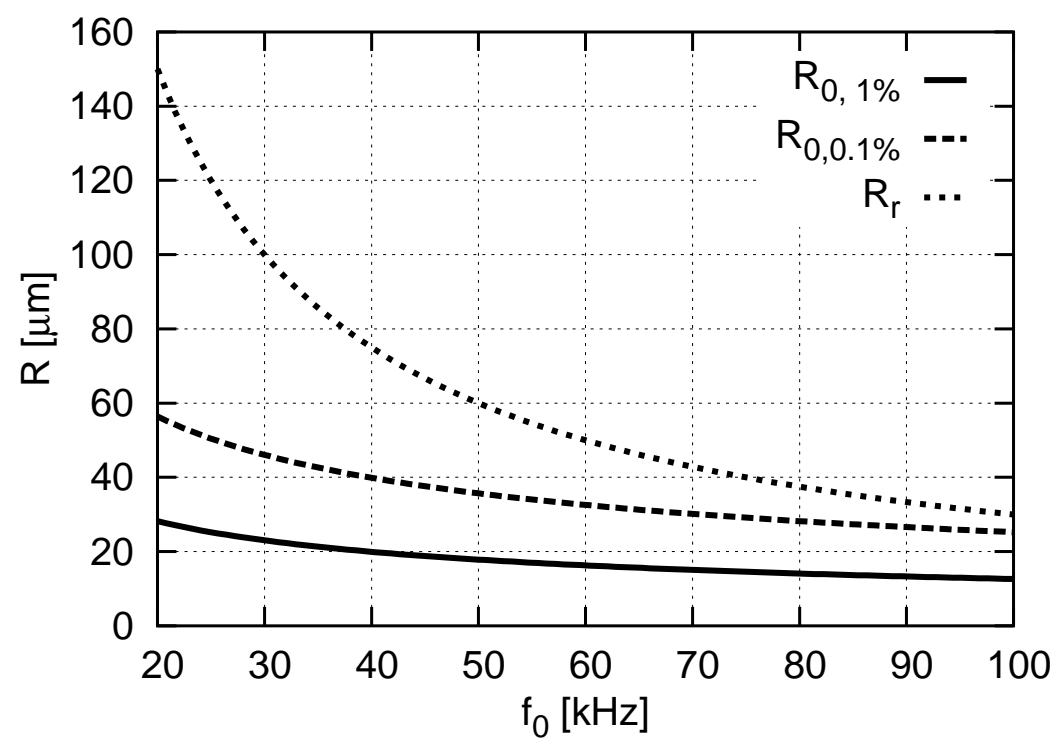

Abbildung 3.3: Resonanzradius (gepunktet), Ruheradius mit $\left|\bar{v}_{T}-v_{T}\right| \leq 10^{-3}$ (oberhalb der gestrichelten Kurve) und $\left|\bar{v}_{T}-v_{T}\right| \leq 10^{-2}$ (oberhalb der durchgezogenen Linie).

\section{Abschätzung des Gültigkeitsbereiches}

Für die praktische Anwendung der Ergebnisse ist es notwendig, den Gültigkeitsbereich der verwendeten Annahmen zu bestimmen.

1. Lineare Blasenschwingung: Einen maximalen Fehler von 1\% erhalten wir, wenn wir eine Auslenkung von maximal $10 \%$ bezüglich des Ruheradius zulassen, das heißt

$$
\epsilon \leq 0.1
$$

Zur Bestimmung der Auslenkung verwenden wir Abschätzung (2.11). Das Ergebnis ist in Abbildung 3.4 dargestellt. Dort ist der Schalldruck $p_{a}$ für $\epsilon=0.1$ in Abhängigkeit vom relativen Ruheradius $R_{0} / R_{r}$ aufgetragen.

2. Hohe Reynoldszahl: Zur Charakterisierung der Bewegung verwenden wir die von Magnaudet und Legendre eingeführte zweite Reynoldszahl $R e_{R}$ (siehe Kapitel 2, Abschnitt 2.2). Für $R e_{R} \geq 12$ ist die Bewegung trägheitsdominiert [72]. Mit der Blasenwandgeschwindigkeit

$$
\dot{R}(t)=-\epsilon \omega R_{0} \cos (\omega t)
$$




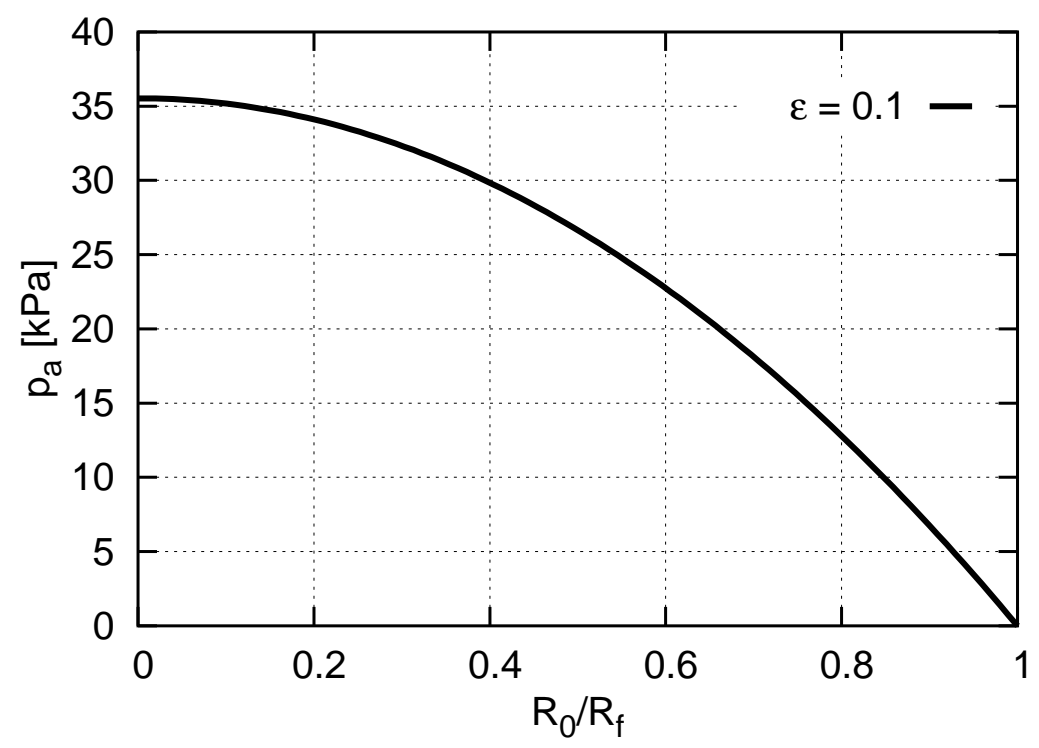

Abbildung 3.4: Schalldruck, bei dem die relative Auslenkung $\epsilon=0.1$ erreicht wird (unterhalb der Kurve ist das Kriterium der Linearisierung erfüllt).

ergibt sich für eine Blase in Wasser

$$
\begin{aligned}
R e_{R} & \approx \frac{\rho R_{0}^{2} \epsilon \omega}{\eta} \\
& \approx 10^{6} R_{0}^{2} \epsilon \omega .
\end{aligned}
$$

Damit folgt die Bedingung

$$
R_{0} \frac{R_{0}}{R_{r}} \geq \frac{2 \cdot 10^{-6}}{\pi \epsilon} \mathrm{m}
$$

für die Gültigkeit von Gleichung (3.5).

Ein Vergleich mit Abb. 3.2 zeigt, dass dieses Kriterium bei maximal zugelassener Relativamplitude $\left(\epsilon=0.1, R_{0}^{2} / R_{r} \geq 6 \mu \mathrm{m}\right)$ mit einem Fehler von etwa $1 \%$ zusammenfällt. Damit kann dieses Kriterium ebenfalls für die Anwendbarkeit der gemittelten Bewegungsgleichung verwendet werden.

Für Mikroblasen haben Reddy und Szeri gezeigt, dass bei einer akustischen Anregung im MHz-Bereich der hier verwendete Ausdruck für die Widerstandskraft näherungsweise auch für kleine Reynoldszahlen $(R e \ll 1$ und $\left.R e_{R} \ll 1\right)$ gilt [91]. Damit sind die präsentierten Ergebnisse auch auf Mikroblasen anwendbar, sofern die Anregung im entsprechenden Frequenzbereich liegt. 


\section{Kapitel 4}

\section{Experimenteller Aufbau und Messmethoden}

Der hier zur Untersuchung akustischer Kavitationsfelder verwendete Messaufbau ist in Abbildung 4.1 dargestellt.

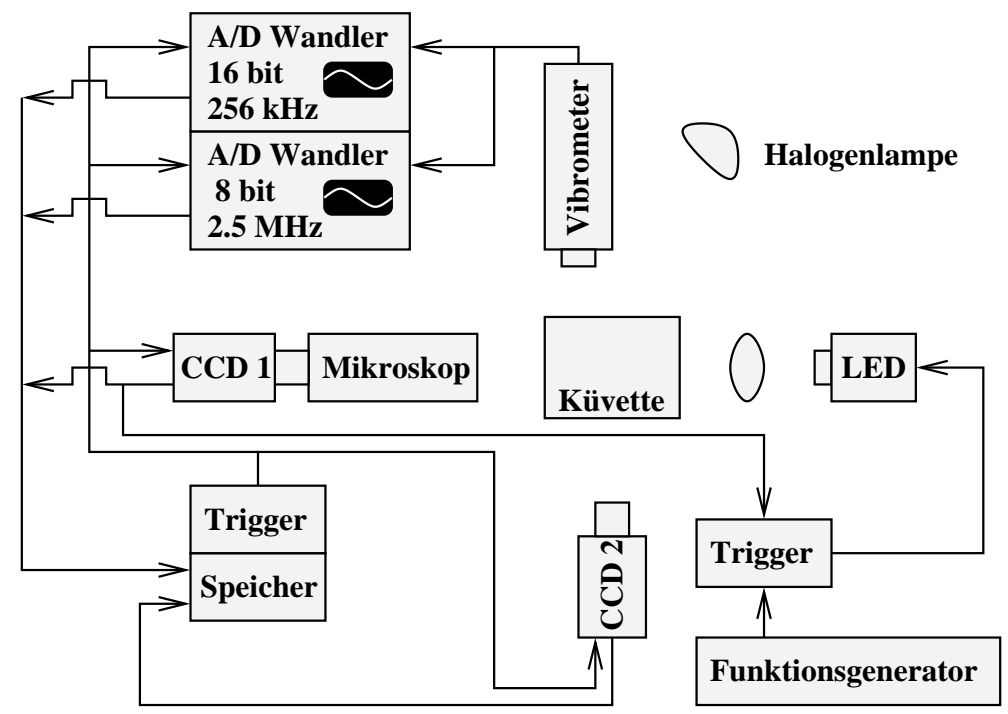

Abbildung 4.1: Experimenteller Aufbau zur Untersuchung akustischer Kavitationsfelder.

Er lässt sich in drei Untersysteme unterteilen:

1. Das schallerzeugende System

2. Das bildgebende System

3. Das System zur Messung der Schallemissionen

Diese Systeme können in ihrer Funktionsweise separat beschrieben werden, im Experiment sind sie jedoch meist miteinander synchronisiert. 


\subsection{Erzeugung des Schallfeldes}

Zur Erzeugung akustischer Stehwellenfelder werden wassergefüllte Quaderküvetten verwendet, die mit einem oder mehreren piezokeramischen Schallwandlern versehen wurden. In Abbildung 4.1 wird das schallerzeugende System vereinfacht durch die Küvette repräsentiert. Im Rahmen dieser Arbeit wurden folgende Systeme verwendet.

1. Glaswürfel

2. Plexiglasquader

3. Plexiglaswanne

4. Ultraschallreaktor

Die Küvetten werden im Anhang einzeln beschrieben. Das Signal wird mit einem Funktionsgenerator ( $h p 33120 \mathrm{~A}$ ) erzeugt und anschließend von einem geeigneten Leistungsverstärker verstärkt. Gegebenfalls wird eine Impedanzanpassung vorgenommen. Abbildung 4.2 skizziert die wesentlichen Teile des schallerzeugenden Systems. Zur Erzeugung der Amplitudenmodulation des Anregungssignals wurden zwei Funktionsgeneratoren gekoppelt.

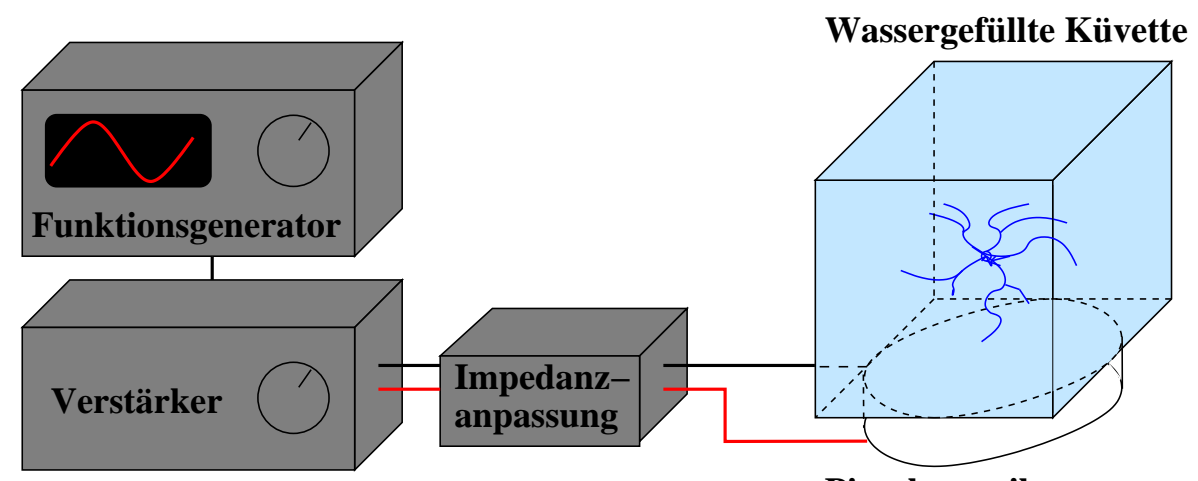

Piezokeramik

Abbildung 4.2: Das schallerzeugende System besteht aus Funktionsgenerator, Leistungsverstärker und einen piezokeramisch getriebenen wassergefüllten Resonator, gegebenenfalls impedanzangepasst. 


\subsection{Bildaufnahme}

Um möglichst viele Zeit- und Raumskalen erfassen zu können, stehen zur Bildaufnahme Kameras unterschiedlicher Auflösung und Schnelligkeit zu Verfügung. Die Spezifikationen der verwendeten Kameras sind in Tabelle 4.1 zusammengestellt.

\begin{tabular}{|l|l|l|}
\hline $\begin{array}{l}\text { Kamera } \\
\text { Name }\end{array}$ & $\begin{array}{l}\text { Auflösung } \\
{[\mathrm{pxl}]}\end{array}$ & $\begin{array}{l}\text { Bildabstand } \\
{[\mathrm{ms}]}\end{array}$ \\
\hline PULNiX TM -6701-AN & $640 \times 480$ & 16.7 \\
\hline HISIS2002 & $256 \times 256$ & 0.4 \\
\hline Imacon 468 & $576 \times 384$ & $1 \mathrm{e}-5$ \\
\hline
\end{tabular}

Tabelle 4.1: Liste der verwendeten Kameras.

Sowohl die PULNiX als auch die HISIS-Kamera sind in zweifacher Ausführung vorhanden und können jeweils miteinander synchronisiert werden. Die HISISKamera kann mit dem derzeit verwendeten Kameratreiber jedoch nicht extern synchronisiert werden, lediglich der Start der Aufnahme kann ausgelöst werden.

Die Kameras können mit verschiedenen Objektiven kombiniert werden, die in Tabelle 4.2 zusammengestellt sind.

\begin{tabular}{|l|l|}
\hline Objektiv & max. Vergrößerung \\
\hline $\begin{array}{l}\text { Computar M6Z1212 } \\
+ \text { Nahlinse }\end{array}$ & 0.6 \\
\hline Rodenstock TL 1-12-215 & 1 \\
\hline Questar QM100 & 10 \\
\hline
\end{tabular}

Tabelle 4.2: Liste der verwendeten Objektive.

So kann die Kavitationsdynamik auf Raumskalen vom Ruheradius einer Sonolumineszenzblase bis zu den Ausmaßen der jeweiligen Küvette untersucht werden. Der Minimalradius einer kollabierenden Blase kann nicht aufgelöst werden. Mit der Imacon 468 können Prozesse auf Zeitskalen bis 10 ns untersucht werden, allerdings ist hier die Aufnahmekapazität auf 8 Bilder beschränkt.

Als Beleuchtung werden je nach Anwendung Streulicht- oder Durchlichtverfahren verwendet. Für Hochgeschwindigkeitsaufnahmen im Durchlicht werden LED-Lampen mit einer Blitzdauer von etwa $5 \mu$ s eingesetzt. Für Aufnahmen mit niedriger Bildwiederholrate (bis maximal $60 \mathrm{~Hz}$ ) kann eine Blitzlampe mit einer Dauer von $1 \mu$ s verwendet werden. Diese Lampen wurden von der elektronischen Werkstatt angefertigt. Für die Ultrahochgeschwindigkeitsaufnahmen wird 
ein kommerzieller Fotoblitz verwendet (Metz SCA 300). Die Streulichtaufnahmen werden mit handelsüblichen Halogenlampen (12 V, 50 Watt) beleuchtet.

Für die Bildverarbeitung werden die von Luther in pvwave implementierten Algorithmen zur Bandpassfilterung, Objekterkennung und -verfolgung benutzt [71, 98]. Durch die Übertragung der Algorithmen in das verwandte Grafikprogramm $i d l$ wurde im Rahmen dieser Arbeit eine grafische Benutzeroberfläche entwickelt, die es erlaubt, die Filterung und das Tracking interaktiv durchzuführen [92].

\subsection{Aufnahme des Schallsignals}

Zur Messung der Schalldruckamplitude können zwei kalibrierte Hydrofone verwendet werden (Reson TC 4038, Bruel und Kjaer 8103). Das Reson TC 4038 ist mit einem Durchmesser von 5 mm etwa halb so breit wie das Bruel \& Kjear 8103. Sein Nachteil ist, dass es sehr empfindlich auf elektrische Störungen reagiert und die Antwort im niederfrequenten Ultraschallbereich stark frequenzabhängig ist.

Zur nichtinvasiven Messung des Schallsignals steht ein Vibrometer (Polytec OFV 303/3001) zu Verfügung. Damit kann die Geschwindigkeit der Küvettenwand an einer ausgewählten Stelle bestimmt werden. Nachteil dieser Messmethode ist, dass auch Wandschwingungen aufgezeichnet werden, die hauptsächlich Informationen über die wandnahe Schallentwicklung enthalten.

Zur Datenaufnahme wurde für die Dauer der Messungen ein 16-bit FFT-Analyzer (ONO SOKKI CF -5220) von der elektronischen Werkstatt zu Verfügung gestellt. Mit ihm können auch geringe Amplitudenanteile im akustischen Spektrum aufgezeichnet werden. Bei einer maximalen Abtastfrequenz von $256 \mathrm{kHz}$ kann jedoch nur das niederfrequente Spektrum untersucht werden.

Die aufgenommenen Zeitreihen werden mit matlab weiterverarbeitet, insbesondere fouriertransformiert [96]. Gegebenenfalls werden die Daten vor der Fouriertransformation gefenstert. 


\section{Kapitel 5 \\ Die Einzelblase}

\subsection{Rückkopplung der Blase auf den Resonator}

Aus schwingungsphysikalischer Sicht werden im akustischen Kavitationsfeld verschiedene Oszillatoren - der Schallwandler, die wassergefüllte Küvette und die Blasen - gekoppelt. Oft jedoch wird das schallerzeugende System nur als externe Anregung der Blase betrachtet und die Kopplung zwischen Küvette und Blase wird als unidirektional angenommen. Insbesondere wird der Schalldruck in einigen Kavitationsexperimenten durch eine Hydrofonmessung im blasenfreien Resonator bestimmt [24, 19, 50, 44, 47]. Die Rückkopplung ist aber nicht immer vernachlässigbar. Der Einfluss der Blase auf die Resonatoreigenschaften beruht zum einen auf der Veränderung des Resonators selber. Das Gasvolumen hat andere akustische Eigenschaften als das umgebende Wasser, die sich auf die mechanischen Eigenschaften des Gesamtsystems auswirken. Die Blasenpulsation selber ist thermisch, viskos und akustisch gedämpft [21]. Die exakte mathematische Behandlung der Kopplung ist bereits in Anwesenheit einer Einzelblase nahezu unmöglich. Sämtliche am Schwingungssystem beteiligte Komponenten und ihre Kopplung müssen bekannt sein. Die numerische Berechnung des Schallfeldes eines Resonators in Anwesenheit eines ortsfesten, nichtschwingenden Körpers ist zwar mit Finite-Elemente-Methoden möglich, aber die nichtlineare Radial- und Translationsdynamik der Blase erschwert das Problem stark. Beide Effekte, die Änderung der Oszillatoreigenschaften des Gesamtsystems als auch die Kopplung in andere Schallfrequenzen können bereits bei Anwesenheit einer Einzelblase gemessen werden. Bei Sonolumineszenzblasen ist eine Verringerung des Schalldrucks in der Nähe der Blase im Vergleich zum blasenfreien Resonator beobachtet worden [73]. Die Änderungen der Schwingungseigenschaften des Resonators in Anwesenheit einer Blase können auch bei niedrigen Anregungsamplituden anhand von Resonanzkurven gemessen werden. 


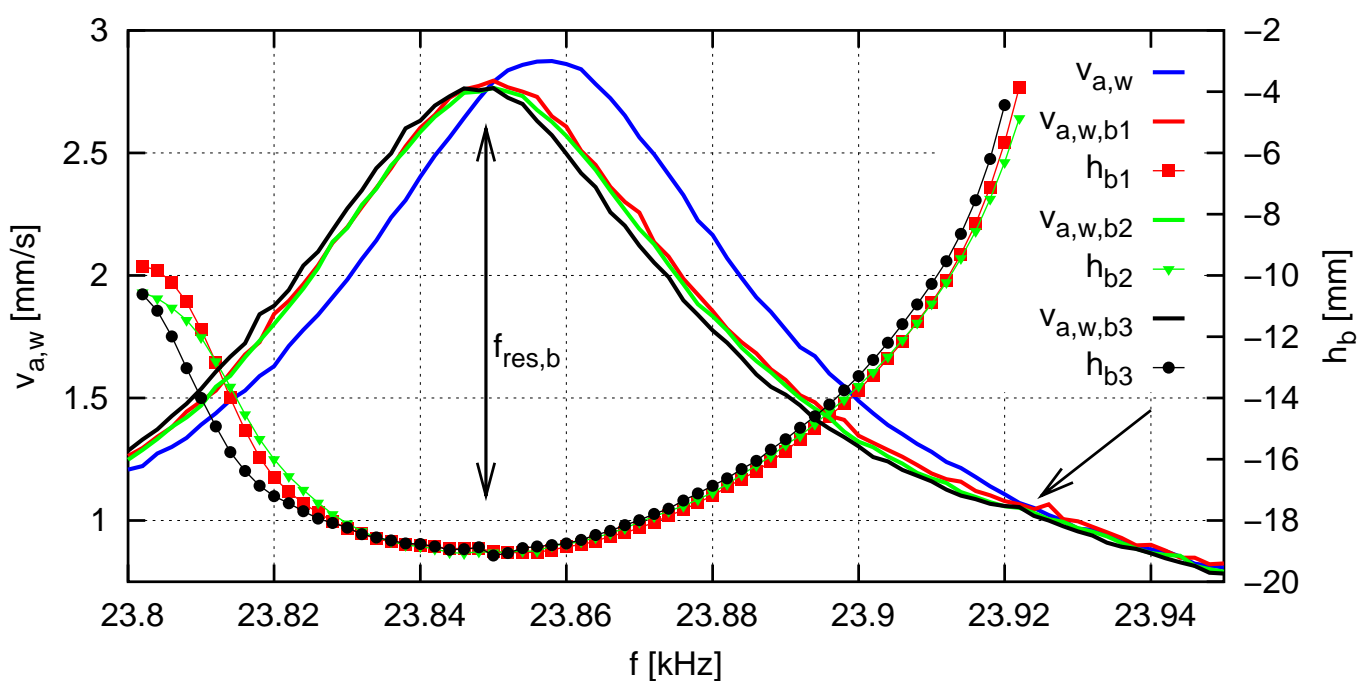

Abbildung 5.1: Resonanzkurve bei einer Anregungsamplitude $U_{a, F G}$ von 1.7 Vpp im blasenfreien Resonator ( $v_{a, w}$, blaue Linie) und bei Anwesenheit einer Einzelblase $\left(v_{a, w, b i}\right)$. Die Symbole geben die vertikale Position der Blase an, wobei $h_{b}=0$ der Wasseroberfläche entspricht.

\subsubsection{Durchführung}

Das Experiment wird im Glaswürfel durchgeführt (siehe Anhang A.1). Zuerst wird die Resonanzkurve des blasenfreien Resonators bestimmt. Bei fester Anregungsamplitude $U_{F G}$ wird die Startfrequenz $f_{t_{0}}$, die unterhalb der Resonanzfrequenz $f_{\text {res }}$ des blasenfreien Schwingungssystems liegt, so gewählt, dass eine Blase im Schallfeld gefangen werden kann. Die Anregungsfrequenz wird in kleinen Schritten erhöht und die Wandgeschwindigkeit $v_{w}$ der Küvette aufgezeichnet. Anschließend wird eine Blase bei der Startfrequenz injiziert und die Messung wiederholt, simultan zum Vibrometersignal wird die Blasenposition gefilmt. Eine Messreihe umfasst mehrere Messungen mit unterschiedlichen Blasen bi. Die Messreihe wird für verschiedene Anregungsamplituden wiederholt. Zum Vergleich werden einige Blasen innerhalb einer Messreihe bei einer Startfrequenz oberhalb der Resonanzfrequenz $\left(f_{t_{0}}>f_{\text {res }}\right)$ injiziert und die Frequenz sukzessive verringert.

\subsubsection{Ergebnisse}

\section{Schwache Anregung}

Abbildung 5.1 zeigt die Geschwindigkeitsamplitude $v_{a, w}$ des Resonators im blasenfreien Zustand und bei drei verschiedenen Realisierungen der Messung mit 
jeweils einer Einzelblase (b1 - b3). Die Anregungsamplitude von $U_{a, F G}=1.7 \mathrm{Vpp}$ entspricht einer Schalldruckamplitude von $15.8 \mathrm{kPa}$ in der Resonanz $f_{\text {res }}$ des blasenfreien Systems. Während sich die Resonanz nicht messbar verbreitert, die Dämpfung also weitgehend gleich bleibt, verschiebt sich die Resonanzfrequenz um etwa $10 \mathrm{~Hz}$. Die zusätzlich eingezeichneten Punkte geben die vertikale Position $h_{b}$ der Blase wieder, ihnen ist die rechte Koordinatenachse zugeordnet. $h_{b}=0$ entspricht dabei der Wasseroberfläche. Mit steigender Amplitude werden die Blasen wie erwartet in den Schalldruckbauch gezogen. Die Blasen erreichen ihre tiefste Position im Resonator in der Resonanz des Gesamtsystems, markiert durch den Pfeil. Mit steigender Frequenz und fallender Amplitude steigen die Blasen auf, bis sie die Wasseroberfläche erreichen. Die Blase ist bereits kurz vorher aus technischen Gründen nicht mehr zu detektieren, deshalb bricht die Trajektorie bereits bei $h_{b}=-4 \mathrm{~mm}$ ab. Erst wenn die Blase bei $\mathrm{f}=23.935 \mathrm{kHz}$ den Resonator verlässt, verläuft die Resonanzkurve wieder wie die des blasenfreien Resonators. Dieses Ereignis ist ebenfalls mit einem Pfeil gekennzeichnet. Die Trajektorien verlaufen für jede Blase etwas unterschiedlich, was auf verschiedene Gleichgewichtsradien hindeutet. Der Unterschied in den Resonanzkurven ist messbar, jedoch ist die Verschiebung im Vergleich zum blasenfreien Resonator deutlich größer als die Verschiebung untereinander.

\section{Mittlere Anregung}

Bei etwas höherer Schallanregung wird der Einfluss der Blasengröße stärker. In Abbildung 5.2 sind einige Messungen bei einer Anregungsamplitude von $U_{F G}=2 \mathrm{Vpp}$ wiedergegeben. Zwei Blasen ( 64 und 65 ) werden bei niedriger Startfrequenz $\left(f_{t_{0}}=23.725 \mathrm{kHz}\right)$, eine weitere Blase $(b 6)$ bei hoher Startfrequenz $\left(f_{t_{0}}=23.85 \mathrm{kHz}\right)$ injiziert. Die Blasen 64 und 65 zeigen qualitativ das gleiche Verhalten wie die Blasen bei der vorigen Messreihe. Allerdings ist hier der Unterschied zwischen den Resonanzkurven größer. Die Blasengröße kann in diesem Experiment nicht aus den Aufnahmen bestimmt werden, da die räumliche Aufösung zu klein ist. Jedoch kann die relative Blasengröße anhand der vertikalen Position bei gleicher Geschwindigkeitsamplitude bestimmt werden.

Bei niedrigen Anregungsamplituden, wenn die Blasenpulsationen nahezu linear verlaufen, werden größere Blasen stärker in den Schalldruckbauch gezogen, die Frequenzunterschiede in der Anregung können hier vernachlässigt werden (siehe Abschnitt 5.2). Blase $b 5$ ist demnach größer als Blase $b 4$, daraus folgt, dass größere Blasen die Resonanzfrequenz stärker verschieben. Blase $b 6$ ist bei der Injektion kleiner als die beiden anderen Blasen bei dieser Frequenz $(f=23.85 \mathrm{kHz})$. Bis 


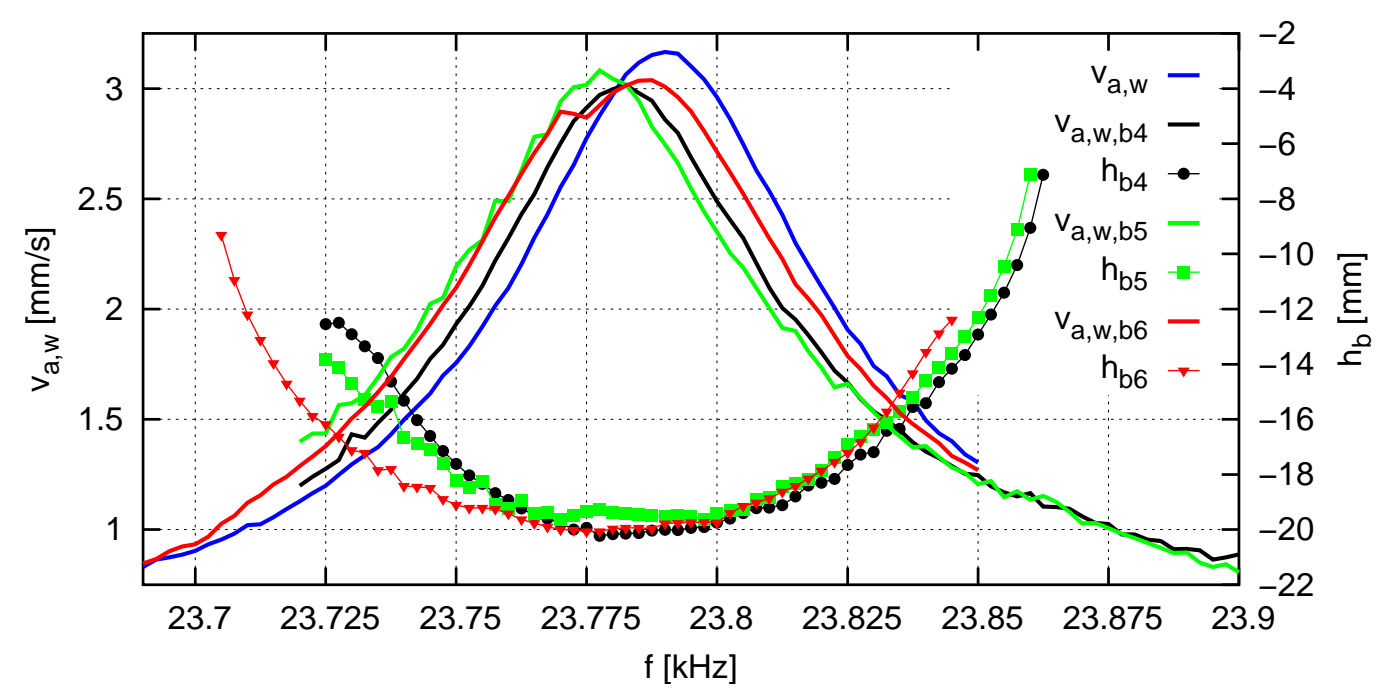

Abbildung 5.2: Resonanzkurve bei einer Anregungsamplitude $U_{a, F G}$ von $2 \mathrm{Vpp}$ im blasenfreien Resonator (gestrichelte blaue Linie) und bei Anwesenheit einer Einzelblase. Die Symbole geben die vertikale Position der Blase an, wobei $h_{b}=0$ der Wasseroberfläche entspricht.

etwa zum Amplitudenmaximum verläuft die Resonanzkurve regulär, die Resonanzfrequenz liegt zwischen der des blasenfreien Systems und der aus Messung b4. Bereits kurz nach Erreichen des Maximums scheint sie sich zu verbreitern. Bei $\mathrm{f}=23.775 \mathrm{kHz}$ macht die Resonanzkurve einen Sprung, während die Trajektorie weitgehend stetig verläuft. Die Resonanzkurve folgt im weiteren Verlauf qualitativ der Resonanzkurve in Anwesenheit der Blase b5, die Blase 66 wird aber deutlich stärker in den Schalldruckbauch gezogen. Der Resonanzverlauf kann mit dem diffusiven Anwachsen der Blasen während der Durchführung der Messung erklärt werden (siehe Abschnitt 2.3) [26]. Alle Blasen wachsen während der Messung an. Vergleicht man ihre Positionen bei zwei Frequenzen gleicher Amplitude zu Beginn und am Ende der Messung, so werden die Blasen zum späteren Zeitpunkt stärker in den Schalldruckbauch gezogen. Blase 65 zum Beispiel befindet sich bei $\mathrm{f}=23.725 \mathrm{kHz}$ und einer Amplitude von $v_{a, w}=1.5 \mathrm{~mm} / \mathrm{s}$ bei $h_{b 5}=-14 \mathrm{~mm}$. Gegen Ende der Messung wird diese Amplitude bei $\mathrm{f}=23.83 \mathrm{kHz}$ erreicht, die Blase befindet sich bei $h_{b 5}=-16 \mathrm{~mm}$. Mit der Größe der Blase verringert sich die Resonanzfrequenz $f_{\text {res }, b}$. Bei steigender Anregungsfrequenz entfernt sich das System immer weiter von der sinkenden Resonanzfrequenz, dies führt im Wesentlichen zu einem schnelleren Abfall der Kurve. Bei einer schrittweisen Verringerung der Frequenz läuft die Resonanzverschiebung in die gleiche Richtung wie die Frequenzverschiebung, die Kurve wird breiter. Der Verlauf der Resonanzkurve ist damit zeitabhängig auf der Skala der Diffusionsgeschwindigkeit. 
Um den Einfluss größerer Blasen zu bestimmen, wird durch Injektion mehrerer Flüssigkeitstropfen eine große Blase erzeugt. In Abbildung 5.3 sind die Resonanzkurven zweier großer Blasen bei steigender Frequenz dargestellt. Zur Erzeugung

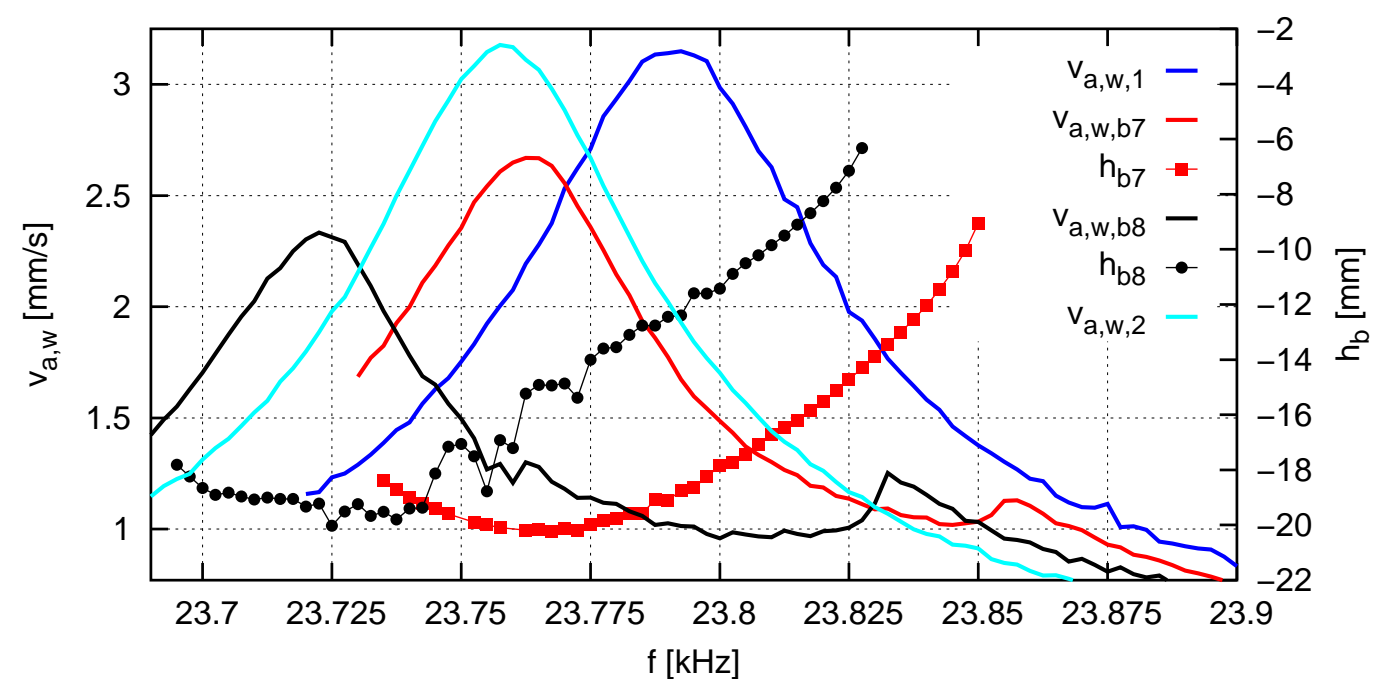

Abbildung 5.3: Resonanzkurve bei einer Anregungsamplitude $U_{a, F G}$ von $2 \mathrm{Vpp}$ im blasenfreien Resonator zu Beginn der Messreihe $\left(v_{a, w, 1}\right)$, nach Beendigung der Messreihe $\left(v_{a, w, 2}\right)$ und bei Anwesenheit einer Einzelblase $\left(v_{a, w, b i}\right)$. Die Symbole geben die vertikale Position der Blase an, wobei $h_{b}=0$ der Wasseroberfläche entspricht.

der Blasen muss relativ viel Wasser eingetropft werden. Da einige Versuche notwendig sind, um eine große Blase bei geeigneten Parameterwerten einzufangen, steigt während dieser Messung der Wasserspiegel merklich. Dies verursacht eine Verringerung der Resonanzfrequenz auch im blasenfreien System. Die Halbwertsbreite und Maximalamplitude verändern sich jedoch nicht. Die blauen Linien geben die Resonanzkurve des blasenfreien Resonators vor und nach den Messungen wieder. Die Verschiebung der Resonanz ist auch am Verlauf der Resonanzkurve nach Verlassen der Blase aus dem Wasservolumen zu erkennen ( $b$ \%: f $>23.86 \mathrm{kHz}$, b8: $\mathrm{f}>23.35 \mathrm{kHz}$ ). Die Verringerung der Resonanzfrequenz durch die Blasen sind hier deutlich stärker als bei den vorigen Messungen, die Maximalamplitude nimmt stark ab, besonders bei Messung b8. Zusätzlich ist hier der Verlauf der vertikalen Position sehr unregelmäßig. Die Blase zeigt ein sehr unstetes Verhalten, das vermutlich durch Oberflächeninstabilitäten verursacht wird (siehe Abschnitt 2.4 und 5.3). In Abbildung 5.4 sind die Trajektorien der beiden Blasen wiedergegeben. Auch in horizontaler Richtung zeigt Blase $b 8$ eine höhere Variabilität als Blase b\%. 


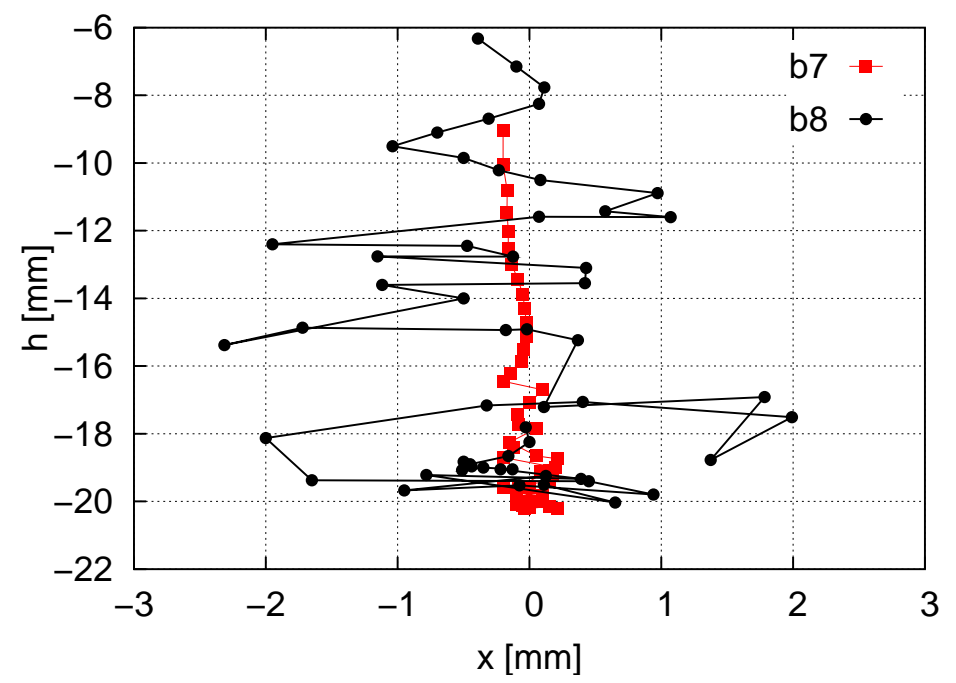

Abbildung 5.4: Trajektorien der Blasen aus Abbildung 5.3.

Bei einer Startfrequenz oberhalb der Resonanzfrequenz des blasenfreien Systems $f_{t_{0}}>f_{\text {res }}$ kann bei großen Blasen kaum eine Resonanzüberhöhung erreicht werden. Abbildung 5.5 gibt die Resonanzkurven und vertikalen Positionen wieder.

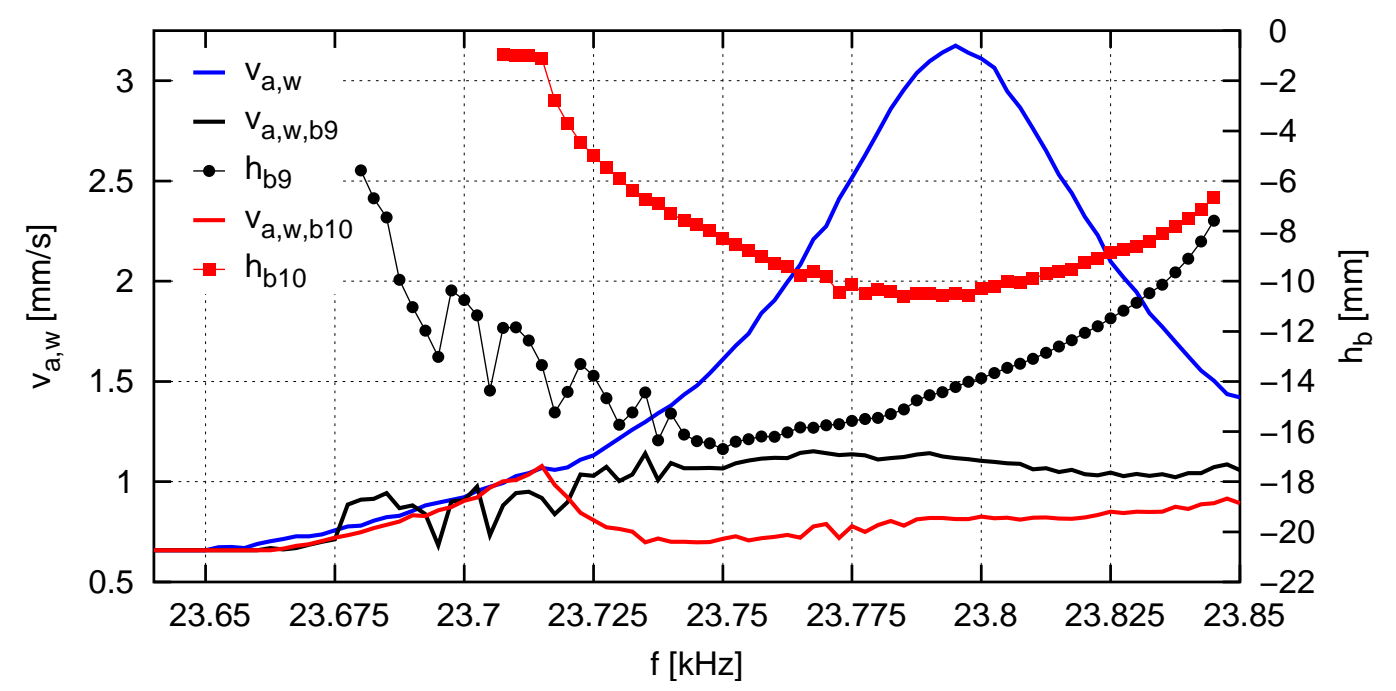

Abbildung 5.5: Resonanzkurven und vertikale Blasenpositionen großer Blasen bei $U_{a, F G}=2$ Vpp. Beschreibung siehe Abb 5.2.

Blase $b 9$ erzeugt noch eine schwache Amplitudenerhöhung bei der Senkung der Anregungsfrequenz und folgt bis zum Amplitudenmaximum qualitativ dem Verlauf der vorherigen Messungen. Dem sehr breiten Amplitudenmaximum folgt dann eine irregulär schwankende Amplitude, wobei der Verlauf qualitativ mit 
der vertikalen Position der Blase korreliert zu sein scheint. Amplitudenminima fallen mit Minima der Blasenposition zusammen. Hier scheint ein Wechselspiel zwischen Größenveränderungen der Blase durch Diffusion und Fragmentation und den damit verbundenen Resonanzverschiebungen, und der Verringerung der Anregungsfrequenz stattzufinden. Bei sehr niedrigen Frequenzen übersteigt die Amplitude dann nochmal die Amplitude des blasenfreien Resonators, die Blase steigt jedoch weiter auf. Blase b10 läuft offensichtlich schneller aus der Resonanz, als es die Verringerung der Anregungsfrequenz kompensieren kann, die Geschwindigkeitsamplitude fällt. Trotzdem wird die Blase durch das Anwachsen geringfügig ins Zentrum der Küvette gezogen, bis sie bei weiterem Fallen der Amplitude aufsteigt. Eine deutliche Resonanzüberhöhung kann bei fallender Anregungsfrequenz dadurch erzielt werden, dass die Messung bei einer tieferen Startfrequenz $\left(f_{t_{0}}=23.74 \mathrm{kHz}, \Delta f<0\right)$ begonnen wird. In Abbildung 5.6 ist das Ergebnis dieser Messung wiedergegeben.

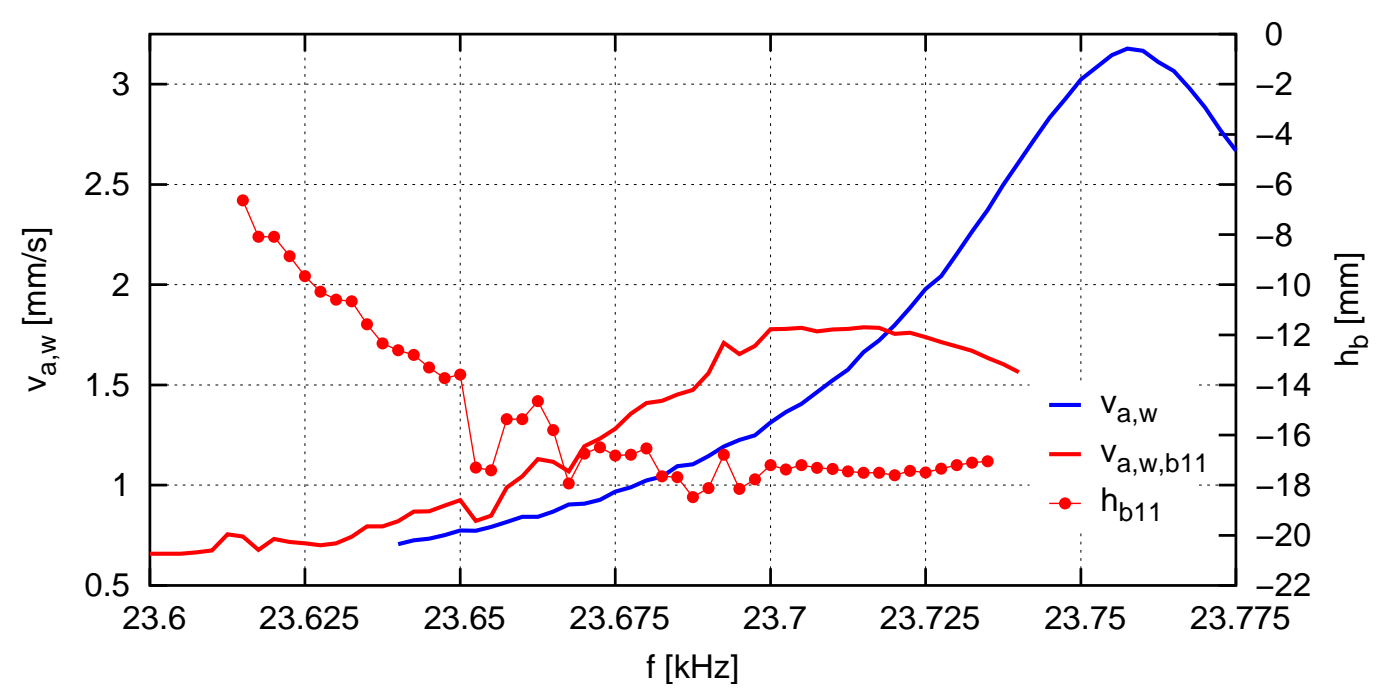

Abbildung 5.6: Resonanzkurve und vertikale Blasenposition einer großen Blase bei $U_{a, F G}=2$ Vpp. Beschreibung siehe Abb 5.2.

Blase $b 11$ wird bei der Injektion $(\mathrm{f}=23.74)$ stärker in die Mitte gezogen $\left(h_{b 11} \approx-17 \mathrm{~mm}\right)$ als die in Abbildung 5.6 wiedergegebene Blase b9. Anschließend ändert sie bei steigender Amplitude ihre vertikale Position kaum. Ab etwa $23.7 \mathrm{kHz}$ abwärts ist der Verlauf von Geschwindigkeitsamplitude und Blasenposition ähnlich wie bei Messung 69 . 


\title{
5.1.3 Hohe Anregungsamplitude
}

Eine weitere Messreihe wird bei einer Anregungsamplitude von $U_{a, F G}=4 \mathrm{VPP}$ durchgeführt. Abbildung 5.7 gibt die Resonanzkurven und vertikalen Positionen von vier verschiedenen Messungen wieder. Der Übersichtlichkeit halber werden hier die vertikalen Blasenpositionen separat dargestellt. Bei Messung b12 - b14

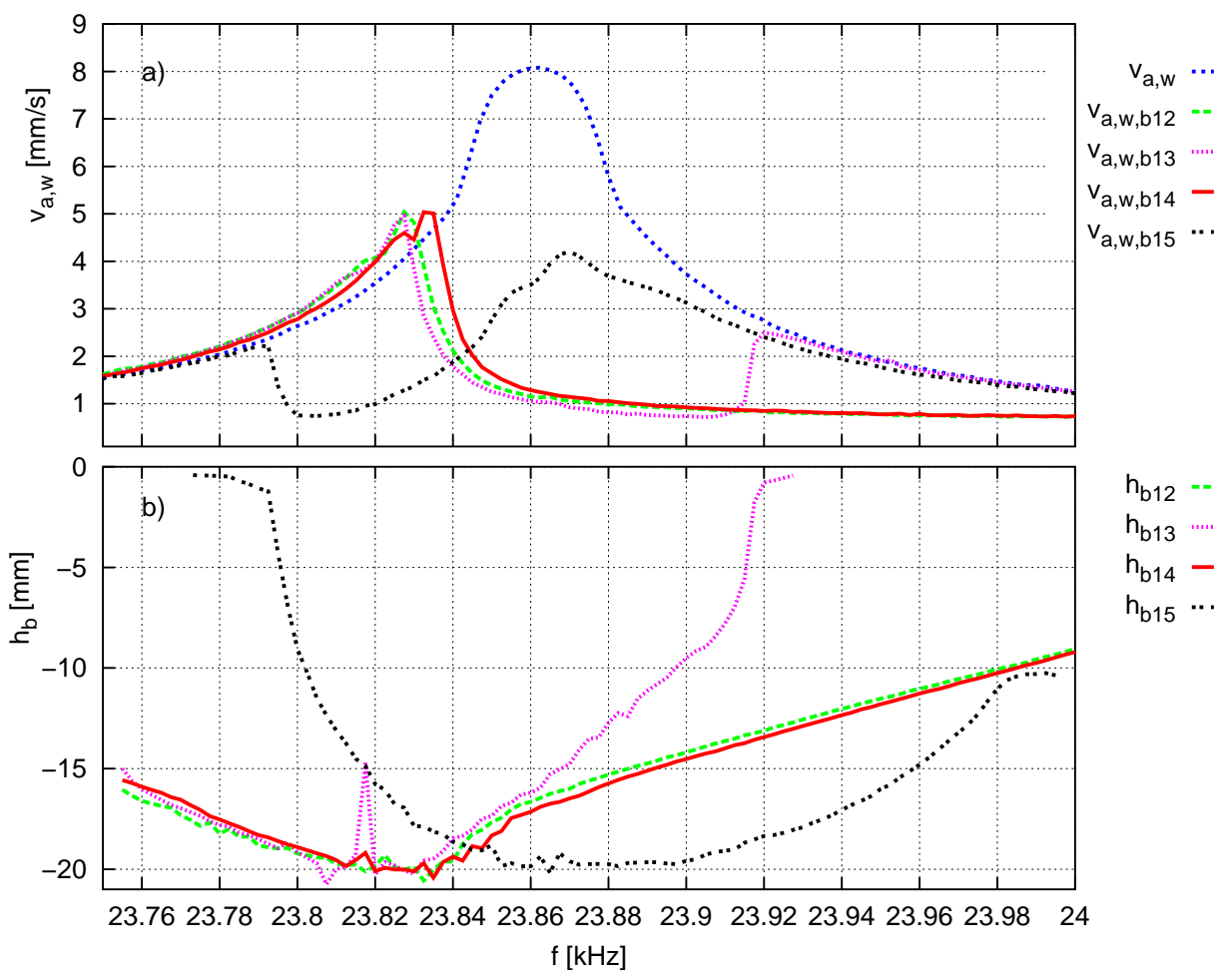

\begin{abstract}
Abbildung 5.7: Resonanzkurve bei einer Anregungsamplitude $U_{a, F G}$ von 4 Vpp. Das obere Bild zeigt die Geschwindigkeitsamplitude im blasenfreien Resonator $\left(v_{a, w}\right)$ und bei Anwesenheit einer Einzelblase $\left(v_{a, w, b i}\right)$. Im unteren Bild sind die vertikalen Positionen der Blasen dargestellt. Der für eine Messung gewählte Linientyp ist in beiden Bildern identisch.
\end{abstract}

wird die Frequenz erhöht. Zuerst verlaufen die Resonanzkurven relativ glatt und steigen an, die Resonanzfrequenz des Systems scheint also höher zu liegen. Die Blasen erreichen den Schalldruckbauch bei $h_{b}=-20 \mathrm{~mm}$, die Amplitude steigt weiter. Dann fällt die Amplitude plötzlich sehr stark. Blase b13 steigt auf, kurz vor Erreichen der Wasseroberfläche nimmt die Amplitude wieder zu. Beim Verlassen der Blase erreicht die Resonanzkurve diejenige des blasenfreien Systems. Blase b12 und 614 bewegen sich mit zunehmender Frequenz nach oben, jedoch sehr langsam, die Amplitude bleibt niedrig. Dieses unterschiedliche Verhalten von großen Blasen wird auch bei Messungen mit konstanter Schallanregung beobach- 
tet. Vermutlich wird die Blase durch Fragmentation kleiner und die Resonanzfrequenz verschiebt sich wieder in Richtung der Anregungsfrequenz. Durch die steigende Schalldruckamplitude wird die Blase zurück ins Resonatorzentrum gezogen. Fragmentiert die Blase nicht (oder nicht stark genug) so steigt sie auf. Bei Messung $b 15$ wird die Frequenz gesenkt. Auch hier verläuft die Resonanzkurve zu Beginn regulär. Die Amplitude steigt etwas weniger schnell als beim blasenfreien Resonator, während die Blase mit zunehmender Amplitude in den Schalldruckbauch gezogen wird. Das Amplitudenmaximum liegt noch oberhalb der blasenfreien Resonanzfrequenz, dann fällt auch hier die Amplitude, jedoch sehr viel flacher. Zeitgleich beginnt die Blase aufzusteigen, wieder ist der Amplitudenanstieg kurz vor dem Erreichen des Wasserspiegels zu beobachten. Das Ergebnis ist im Einklang mit den vorigen Messungen. Die Blase wächst bei hohen Amplituden sehr schnell an, so dass der Resonator schneller aus der Resonanz läuft, als die Frequenzverringerung erfolgt. Als weiterer Effekt kommt hinzu, dass die Blase in diesem Amplitudenbereich ihre Größe durch Fragmentationsprozesse ständig verändert, so dass der Verlauf der aktuellen Resonanzfrequenz des Systems weder stetig noch monoton verläuft.

Zusammenfassend lässt sich feststellen, dass bereits eine Einzelblase einen signifikanten Einfluss auf die Schwingungseigenschaften des Gesamtsystems hat. Dabei spielt die Dämpfung eine untergeordnete Rolle, vermutlich wirkt sich die Anwesenheit der Blase am stärksten auf die Rückstellkraft des Systems aus. Je größer die Blasen sind, desto niedriger ist die Resonanzfrequenz. Durch die Anwesenheit einer Blase kann die Amplitude der Wandgeschwindigkeit im Vergleich zum blasenfreien Resonator stark verringert werden. Die Wechselwirkungsprozesse zwischen Resonanzfrequenz des Systems, Diffusions- und Fragmentationsprozessen sowie der Position der Blase im Schallfeld sind dabei komplex und können zu einem irregulären Verlauf der Resonanzkurve führen. Die Messungen wurden an der Küvettenwand durchgeführt. Die direkte Übertragung der Ergebnisse auf die Schalldruckverhältnisse im Resonator kann nur unter der Annahme gemacht werden, dass die Proportionalität zwischen Wandgeschwindigkeit und Schalldruckamplitude im Küvettenzentrum auch in Anwesenheit der Blase erhalten bleibt. Diese Annahme wird gestützt durch Beobachtungen bezüglich der Blasenposition und dem Verhalten von großen Blasen bei konstanter Anregungsamplitude. Nach dem Aufsteigen einer oberflächeninstabilen Blase formieren sich die verbleibenden Mikroblasen erst dann wieder zu einer Blase, wenn die große Blase den Resonator verlassen hat, in diesem Moment steigt auch die Amplitude der Wandgeschwindigkeit sprunghaft. Auch Streamerstrukturen können ähnliches Verhalten zeigen (siehe Kapitel 7, Abschnitt 7.2.3). 


\subsection{Translation}

\subsubsection{Trajektorien von Einzelblasen}

\section{Aufsteigende Blasen im schwachen Schallfeld}

Zur Überprüfung vorhandener Modelle zur Beschreibung der Translationsdynamik (siehe Abschnitt 2.2) wird das Aufsteigen von Einzelblasen bei verschiedenen Anregungsamplituden im akustischen Stehwellenfeld aufgezeichnet und mit der Theorie verglichen. Ähnliche Untersuchungen wurden bereits 1970 von Crum und Eller durchgeführt [19]. Allerdings wurden nur Blasen untersucht, die tatsächlich im Schallfeld gehalten werden. Die Radienbestimmung über die Aufstiegsgeschwindigkeit setzt Annahmen über die geltende Reibungskraft voraus. Zudem basiert das theoretische Modell auf der Annahme, dass die Stokes-Reibung für harte Kugeln anwendbar ist. Die Theorie bezüglich der viskosen Widerstandskraft bei der Translation ist in den letzten Jahren weiterentwickelt worden (siehe Abschnitt 2.2). Eine experimentelle Überprüfung dieser Theorien wurde bisher noch nicht durchgeführt.

\subsubsection{Durchführung}

Im Glaswürfel (siehe Anhang A.1) wird eine Blase bei einer Anregungsamplitude von $U_{F G}=2 \mathrm{Vpp}$ im Schallfeld gehalten. Der Fokus des Fernmikroskops wird zur Bestimmung des Radius auf eine Stelle oberhalb der aktuellen Blasenposition gerichtet. Dann wird die Amplitude gesenkt und im Anschluss die Aufnahme der beiden Kameras und des 16-bit A/D-Wandlers gestartet. Die Messung wird für verschiedene Anregungsamplituden $U_{a, F G}$ durchgeführt. Anschließend wird die Messung bei konstanter Anregungsamplitude für verschiedene aufsteigende Blasen wiederholt.

\subsubsection{Ergebnisse}

Abbildung 5.8 (a) gibt die vertikale Position von Blasen unterschiedlichen Radius $R_{0}$ bei verschiedenen Anregungsamplituden wieder. Die mittlere Schalldruckamplitude $p_{a}$ wurde aus der Wandgeschwindigkeit bestimmt (siehe Anhang A.1). Abbildung (b) zeigt die Aufstiegsgeschwindigkeiten $v_{z}$ der Blase in Abhängigkeit von der vertikalen Position z. Der Schalldruckbauch liegt etwa bei z $=-22 \mathrm{~mm}$, bezogen auf die Wasseroberfläche bei $\mathrm{z}=0$. 

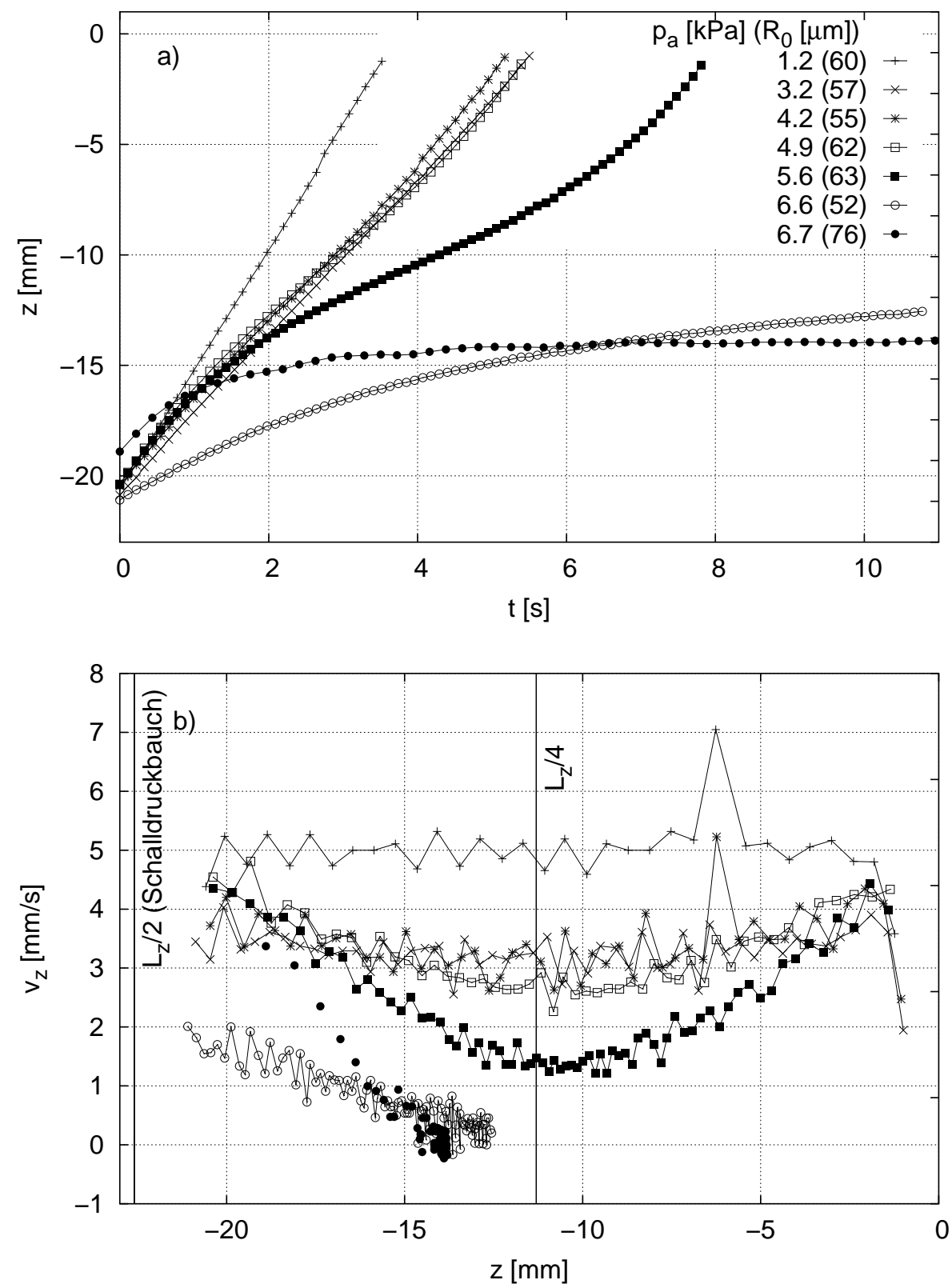

Abbildung 5.8: Vertikale Position $\mathrm{z}(\mathrm{t})$ aufsteigender Blasen bei verschiedenen Anregungsamplituden (a) und Aufstiegsgeschwindigkeit $v_{z}$ in Abhängigkeit von z (b). Die Legende gibt die jeweilige mittlere Schalldruckamplitude $p_{a}$ und den Blasenradius $R_{0}$ an. Der Druckbauch liegt etwa bei $\mathrm{z}=-22 \mathrm{~mm}$, die Wasseroberfläche befindet sich bei $\mathrm{z}=0$. 

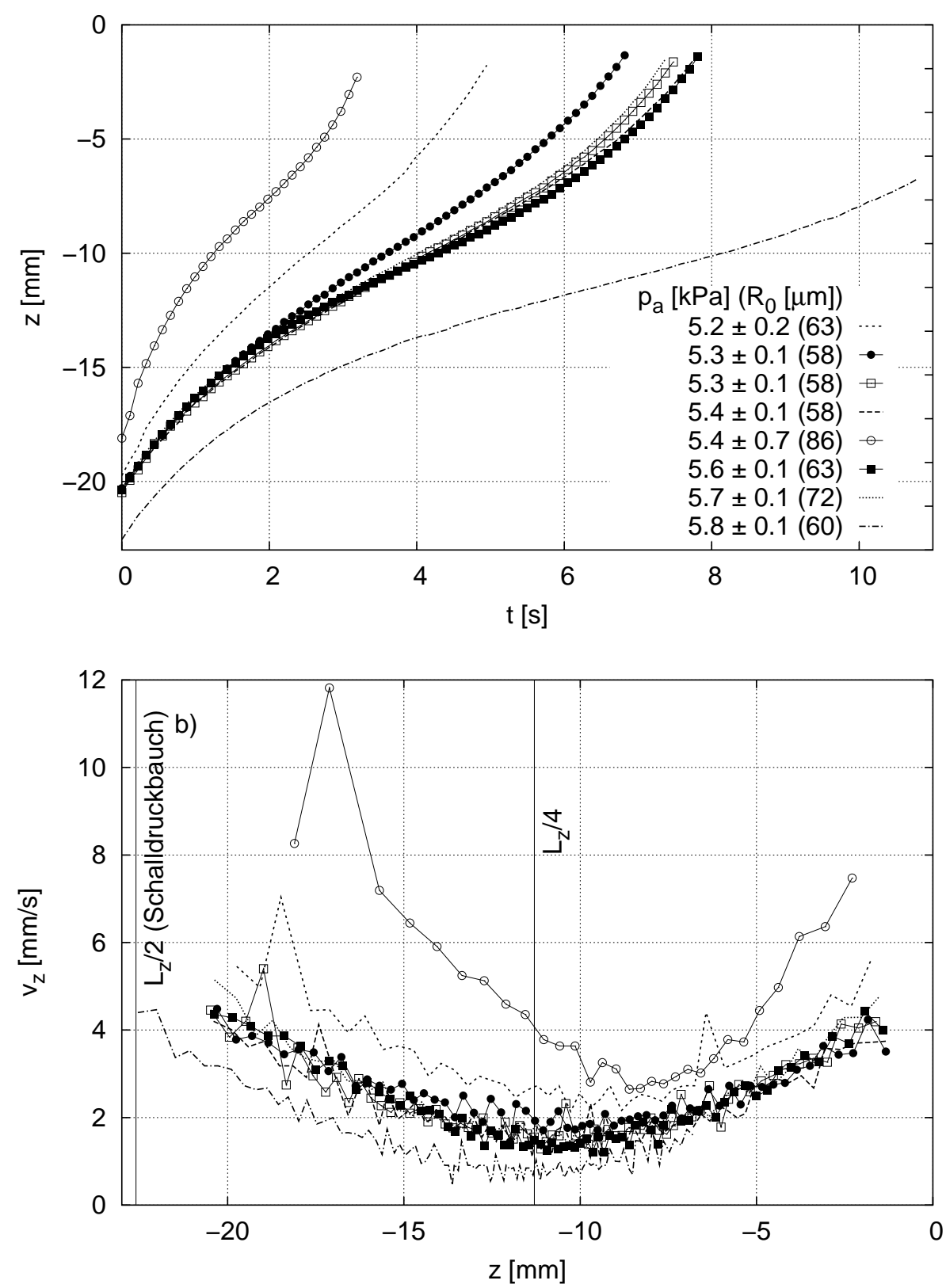

Abbildung 5.9: Vertikale Position z aufsteigender Blasen bei konstanter Anregungsamplitude $U_{a, F G}$ (a) und Aufstiegsgeschwindigkeit $v_{z}$ in Abhängigkeit von z (b). Skalierung und Legende analog zu Abbildung 5.8 . 
Mit zunehmender Schalldruckamplitude nimmt die Aufstiegsgeschwindigkeit ab und wird stärker vom Schallfeld beeinflusst. Während die Blase bei $p_{a}=1.2 \mathrm{kPa}$ mit etwa konstanter Geschwindigkeit aufsteigt, zeigt sich bei höherem Schalldruck auf der Hälfte der Strecke zwischen Schalldruckbauch und Wasseroberfläche ein deutliches Geschwindigkeitsminimum. Bei einer weiteren Erhöhung der Anregungsamplitude wird die Blase in einer Position oberhalb des Schalldruckbauches gehalten.

In Abbildung 5.9 sind die Ergebnisse für die Messreihe bei konstanter Anregungsamplitude $\left(U_{a, F G}=0.7 \mathrm{Vpp}\right)$ analog zu Abbildung 5.8 dargestellt. Trotz konstanter Anregungsamplitude variiert die ermittelte Schalldruckamplitude. Dies liegt zum einen an leichten Resonanzverschiebungen des blasenfreien Resonators. Wesentliche Faktoren dürften dabei Schwankungen in der Wassertemperatur und die Erhöhung des Wasserspiegels sein. Die Resonanzfrequenz des blasenfreien Resonators wurde anhand maximaler Wandschwingung vor jeder Blaseninjektion auf $10 \mathrm{~Hz}$ genau neu bestimmt. Zum anderen resultiert sie aber auch aus dem unterschiedlichen Einfluss der Blasen auf das Schallfeld (siehe Abschnitt 5.1).

Lineare Beschreibung Angesichts der niedrigen Schalldruckamplitude scheint ein linearer Ansatz für die Blasenschwingung gerechtfertigt (siehe Abschnitt 2.1.3). Dies ermöglicht eine vereinfachte Behandlung der Translation. Da die Blasen vergleichsweise groß sind, kann die einfach Mittelung der Bewegungsgleichung nach Gleichung (3.11) mit einem Fehler in der Translationsgeschwindigkeit unter 1\% durchgeführt werden (siehe Kapitel 3, Abbildung 3.3). Insbesondere kann der Ausdruck für die primäre Bjerkneskraft analytisch gelöst werden. Da die primäre Bjerkneskraft proportional zum Produkt aus Schalldruck (da direkt proportional zur Auslenkung der Blase) und Schalldruckgradient ist, wird sie auf halber Strecke zwischen Schalldruckbauch und -knoten maximal $\left(z=L_{z} / 4\right)$ (siehe auch Abschnitt 2.2.3). Dort wird also eine Blase am ehesten eingefangen [23]. Dieser Effekt führt zu den Geschwindigkeitsminima der Trajektorien (vgl. Abbildungen 5.8 und 5.9).

\subsubsection{Numerisches Modell}

Die Translationsdynamik wird durch numerische Integration der gemittelten linearisierten RPNNP-Gleichung simuliert. Dabei wird eine radiusabhängige polytrope Expansion angenommen [89, 18]. Die zeitliche Entwicklung der Schalldruckamplitude durch die Anwesenheit der Blase wird in die Simulation implementiert. Sie wird anhand der gemessenen Wandgeschwindigkeit bestimmt und 
durch ein Polynom zweiter Ordnung genähert. Für Integrationszeiten oberhalb der Messdauer des A/D-Wandlers (4 s) wird die Schalldruckamplitude konstant auf den letzten gemessenen Wert gesetzt. Die Bewegungsgleichung wird für verschiedene Reibungskraftmodelle aufgestellt (siehe Abschnitt 2.2) und gelöst. Verwendet wird der in pvwave zu Verfügung gestellte ODE - Algorithmus [98].

\subsubsection{Ergebnisse}

In Abbildung 5.10 (oben) sind die Aufstiegsgeschwindigkeiten zweier Blasen gleicher Größe bei gleicher Schalldruckamplitude, sowie die Ergebnisse der Simulation bei Verwendung verschiedener Modelle für die Reibungskraft aufgetragen.

Alle Modelle geben den Verlauf der Aufstiegsgeschwindigkeit qualitativ wieder, jedoch beschreibt keines die absoluten Werte befriedigend. Die Reibungskraft für hohe Reynoldszahlen (Magnaudet-Legendre) überschätzt die Reibung, die anderen Modelle überschätzen die Geschwindigkeit. Die Reynoldszahl der Translation liegt bei dieser Beispielmessung bei $0.08<R e<0.3$, also im Bereich zwischen den beiden Grenzfällen sehr großer und sehr kleiner Reynoldszahl. Die Abweichungen von den empirischen Ansätzen sind physikalisch erklärbar. Die empirischen Ansätze sind für nichtschwingende Blasen aufgestellt worden, die die zusätzliche durch die Pulsationen verursachte Reibung nicht berücksichtigen. Die Geschwindigkeit hat einen nahezu symmetrischen Verlauf um $\mathrm{z}=L_{z} / 4$, während die Schalldruckamplitude und damit auch die Reynoldszahl der Pulsation in Richtung Wasseroberfläche kontinuierlich abnimmt. Dies spricht dafür, dass die Pulsation in diesem Schalldruckbereich keinen großen Einfluss auf die Reibungskraft hat. Auf der anderen Seite geben die empirisch ermittelten Reibungskraftmodelle die Bewegung nicht korrekt wieder, das heißt, dass der Einfluss der Pulsation wiederum nicht vernachlässigbar ist. Die gemessene Trajektorie mit der höchsten Reynoldszahl, eine vergleichsweise große und schnell aufsteigende Blase $\left(R_{0}=86 \mu \mathrm{m}\right)$, ist in Abbildung 5.10 (unten) wiedergegeben. Die Aufstiegsgeschwindigkeit wird erstaunlicherweise sehr schlecht durch die Reibung bei hohen Reynoldszahlen beschrieben. 

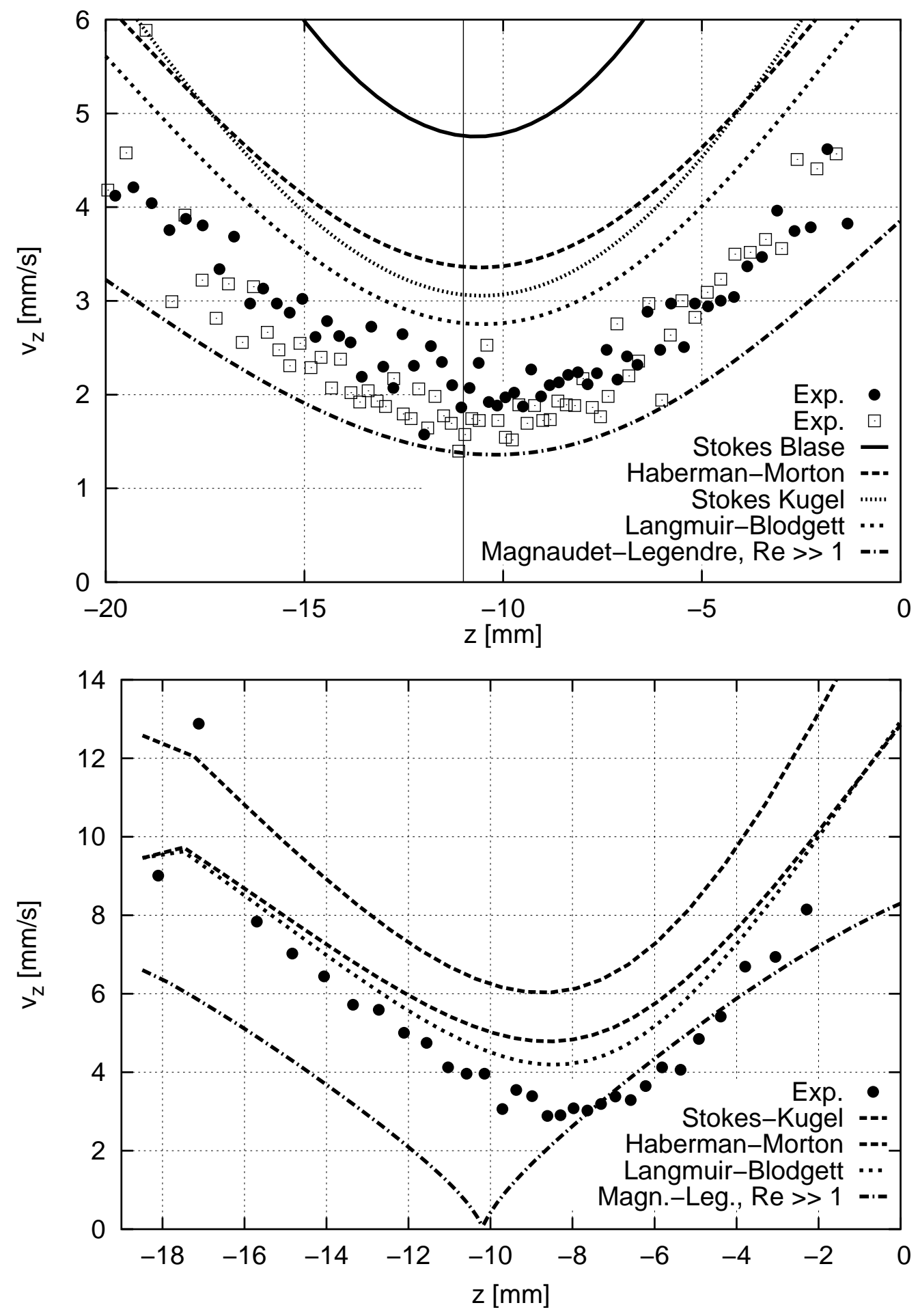

Abbildung 5.10: Vertikale Position und Auftriebsgeschwindigkeit einer Blase mit einem Gleichgewichtsradius $R_{0}=58 \mu \mathrm{m}$ bei einer Schalldruckamplitude von $p_{a} \approx 5.3 \mathrm{kPa}$ (oben) bzw. $R_{0}=86 \mu \mathrm{m}$ und $p_{a} \approx 5.4 \mathrm{kPa}$ (unten). Zwei unterschiedliche Blasen (Symbole) und numerische Simulationen bei Verwendung verschiedener Ansätze für die Reibungskraft. Die vertikale Linie bezeichnet $\mathrm{z}=L_{z} / 4$. 
Qualitativ geben die empirischen Modelle hier die besten Ergebnisse, obwohl auch die Pulsationsgeschwindigkeit wegen der größeren Auslenkung hier höher ist. Nach Magnaudet und Legendre sind nicht nur die jeweiligen Reynoldszahlen $R e, R e_{R}$ wesentlich für die Reibungskraft, sondern auch das Verhältnis von Pulsations- und Translationsgeschwindigkeit $R e_{R} / R e$ [72]. Abbildung 5.11 gibt diese Werte für die beiden Messungen wieder. Die Reynoldszahlen der Translation sind direkt aus den gemessenen Geschwindigkeiten ermittelt, die Reynoldszahl der Pulsation ist aus der Lösung der linearisierten RPNNP-Gleichung und dem ortsabhängigen Schalldruck bestimmt.

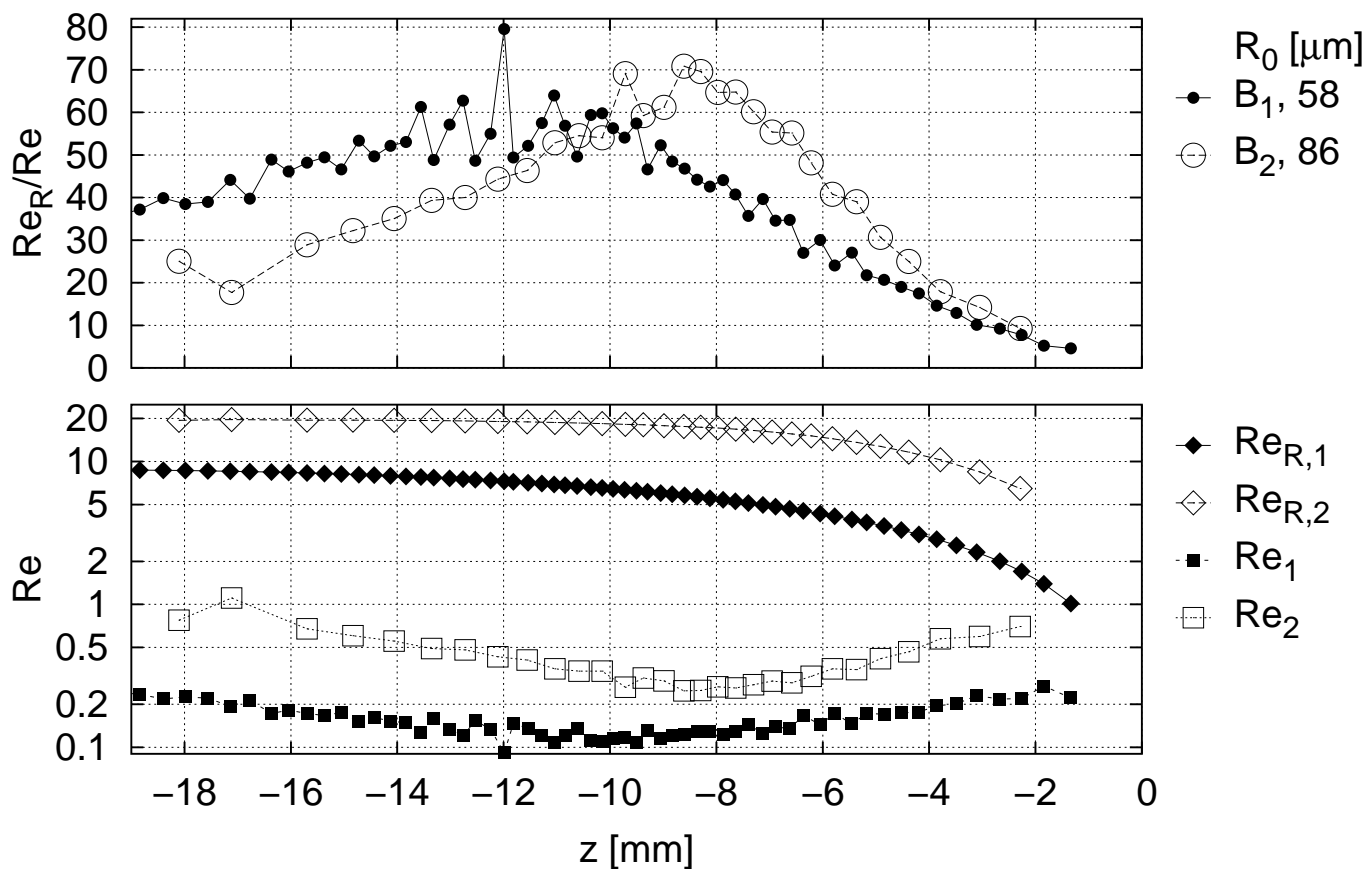

Abbildung 5.11: Vergleich der charakteristischen Geschwindigkeiten der Messungen aus Abb. 5.10. Reynoldszahl der Translation Re und Pulsation $R e_{R}$ (unten) und das Verhältnis von Pulsations- und Translationsgeschwindigkeit (oben).

Die Pulsationsgeschwindigkeit liegt um ein bis zwei Größenordnungen über der Translationsgeschwindigkeit. Bemerkenswert ist, dass in beiden Messungen die Reibungskraft für hohe Reynoldszahlen am besten fittet, wenn das Verhältnis der Geschwindigkeiten groß ist. Dies ist konsistent mit numerischen Lösungen der instationären Navier-Stokesgleichung für aufsteigende schwingende Blasen in unterkühlten Flüssigkeiten, die ebenfalls im mittleren Reynoldszahlenbereich eine stärkere Reibungskraft für stärker oszillierende Blasen ergaben [72]. Da die 
experimentellen Ergebnisse im Einklang mit den theoretischen Vorhersagen stehen, scheint für Blasen in starken Schallfeldern die Näherung der Reibungskraft bei hohen Reynoldszahlen eine gute Beschreibung zu sein, da insbesondere die Blasenwandgeschwindigkeit stark zunimmt.

Nichtlineare Effekte Trotz gleicher äußerer Parameter weicht die Aufstiegsgeschwindigkeit der beiden Blasen, deren Trajektorie simuliert wurde (Abbildung 5.10), deutlich voneinander ab (siehe Abb. 5.9, schwarze Punkte und weiße Quadrate), während Blasen mit leicht unterschiedlichen Parametern ähnliche Aufstiegsgeschwindigkeiten haben (weiße und schwarze Quadrate). In Abbildung 5.12 sind die zweidimensionalen Projektionen der Trajektorien der entsprechenden Blasen zu sehen. Hier sind vergleichsweise kleine aber deutliche Abweichungen

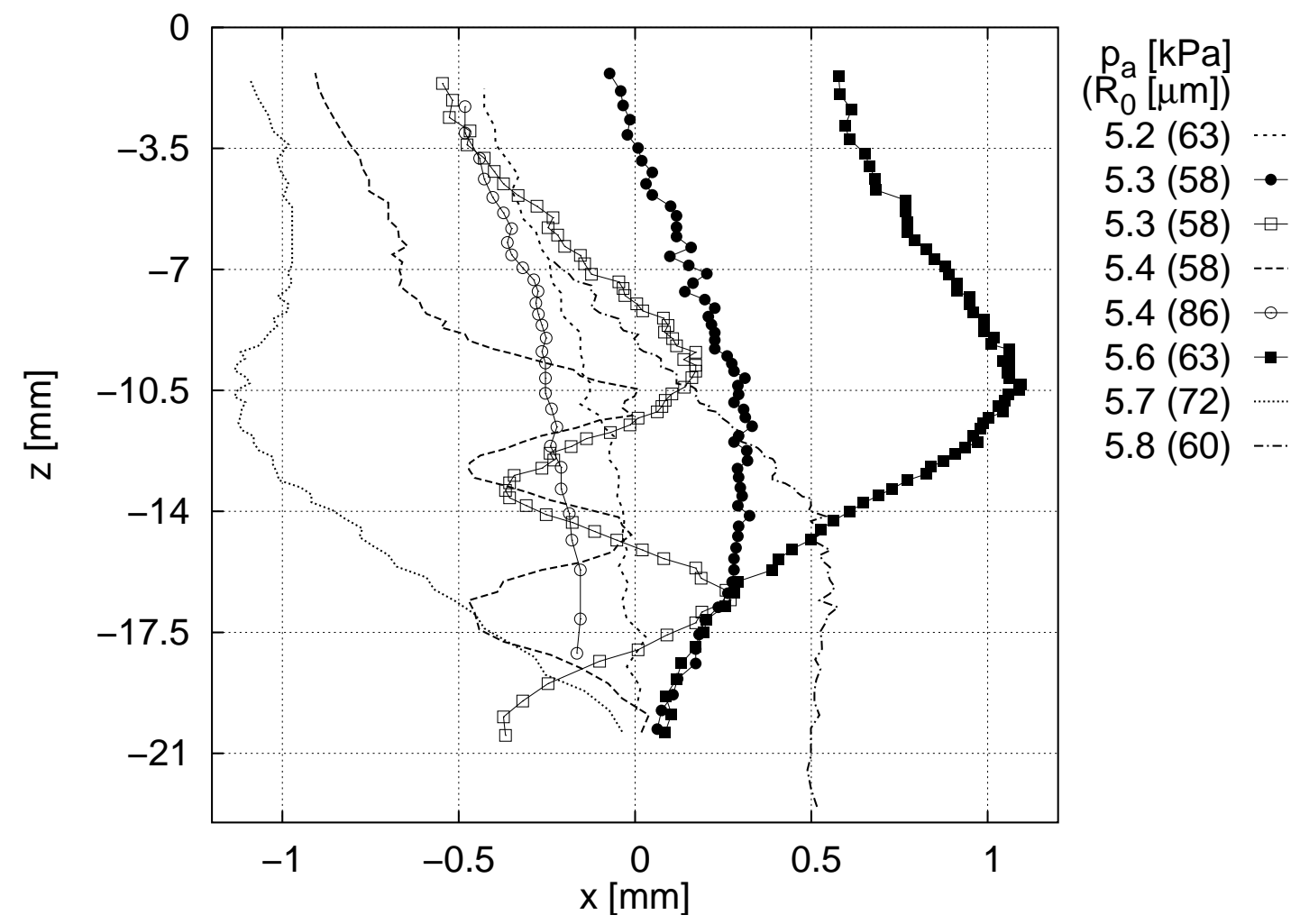

Abbildung 5.12: Zweidimensionale Trajektorien der aufsteigenden Blasen

von der erwarteten konstanten horizontalen Position zu beobachten. Dabei handelt es sich offensichtlich nicht um erratische Bewegungen, sondern einige Blasen scheinen eine oszillatorische Bewegung auszuführen, deren Umkehrpunkte mit dem Schallfeld korreliert sind. Vergleicht man die Trajektorien mit den Aufstiegs- 
zeiten (Abb. 5.9), so beobachtet man, dass die stärker horizontal ausgelenkten Blasen langsamer aufsteigen. Qualitativ lässt sich dieses Verhalten mit der Wechselwirkung zwischen Blasenschwingung und höheren Harmonischen im Schallfeld erklären (siehe auch Anhang A.1). Für lineare Blasenschwingungen verschwindet die primäre Bjerkneskraft für höhere Harmonische der Anregungsfrequenz (siehe Abschnitt 2.2.3. Dies bedeutet, dass bereits bei diesen sehr kleinen Anregungsamplituden der nichtlineare Anteil der Blasenschwingung einen messbaren Einfluss hat. Das Anschwingen der höheren Resonatormoden scheint dabei auch von zufälligen Störungen abhängig zu sein, da es keinen einfachen Zusammenhang zwischen horizontaler Auslenkung und der Schalldruckamplitude oder dem Blasenradius zu geben scheint, wenn auch tendenziell die Auslenkung bei höherer Schalldruckamplitude größer ist. Eine quantitative Simulation dieser Effekte würde die Berücksichtigung der höheren Harmonischen sowohl in der Anregung als auch in der Pulsation der Blase erfordern. Qualitativ kann die horizontale Auslenkung durch einfache Addition einer zusätzlichen Bjerkneskraft mit attraktiven oder repulsiven Fixpunkten an den Stellen der jeweiligen Schalldruckbäuche der höheren Harmonischen reproduziert werden. Abbildung 5.13 zeigt numerische Trajektorien einer Blase im schwachen Schallfeld $\left(R_{0}=60 \mu \mathrm{m}, p_{a}=5 \mathrm{kPa}\right)$ im Resonator mit zusätzlichen höheren Harmonischen. Die Schalldruckbäuche der gradzahligen höheren Harmonischen sind dabei als attraktiv, die der ungradzahligen als repulsiv angenommen worden, um Abweichungen von der horizontalen Achse zu erzeugen.

Die höherfrequenten Oszillationen der Blase können auch durch Störungen oder Stöße ausgelöste Oszillationen der gesamten Flüssigkeit sein, in der die Blase mitbewegt wird. In diesem Fall sollte die horizontale Auslenkung jedoch eine harmonische Funktion der Zeit sein. Abbildung 5.14 zeigt die normierte Horizontaloszillation einmal als Funktion der Vertikalposition (weiße Quadrate) und als Funktion der Zeit (schwarze Quadrate). Ein Vergleich mit einer an die erste Steigung angefitteten Sinusfunktion zeigt, dass die Horizontalauslenkung als Funktion der Vertikalposition der Blase harmonischer verläuft als die Zeitfunktion. Dies ist ein Indiz dafür, dass es sich um eine höhere Resonatormode handelt, in diesem Fall die 13. Harmonische. 


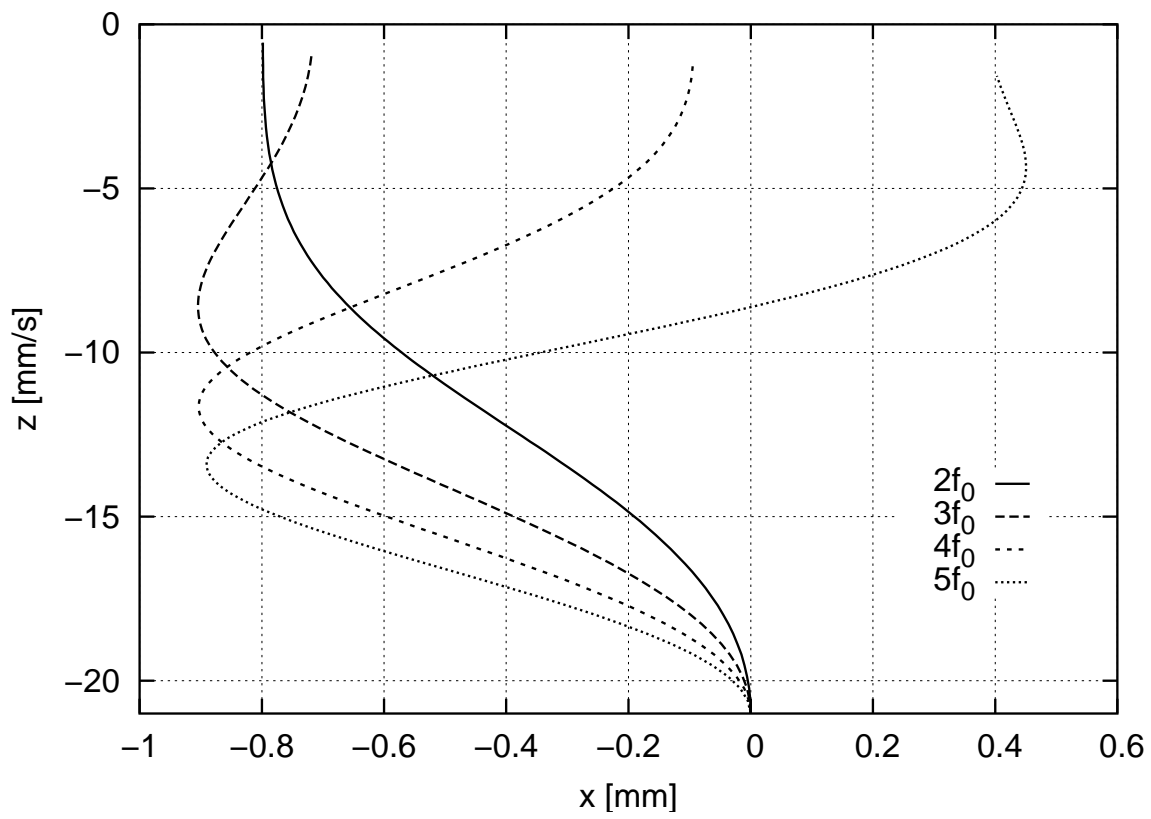

Abbildung 5.13: Einfache Simulation von Trajektorien einer aufsteigenden Blase im Resonator in Anwesenheit von höheren Harmonischen.

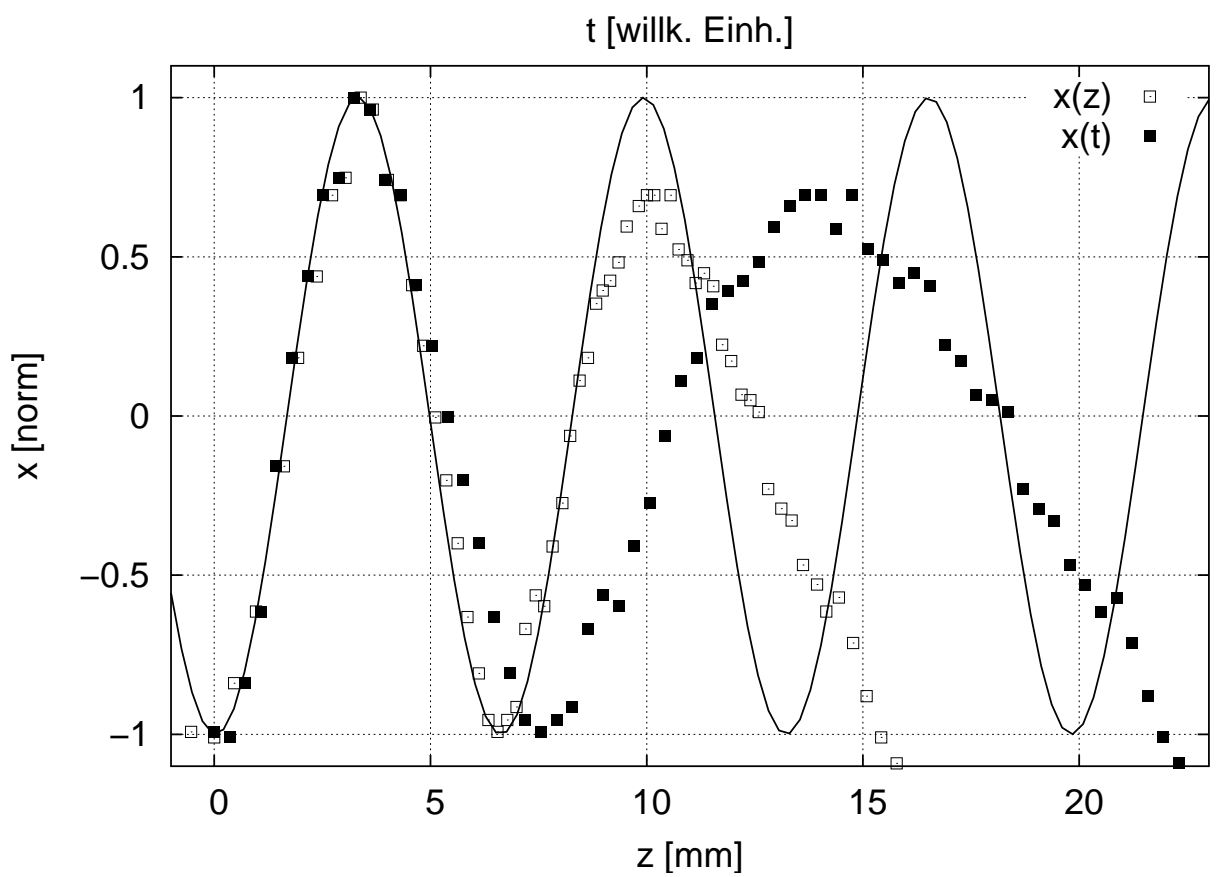

Abbildung 5.14: Die normierte Horizontalauslenkung der Blase als Funktion der Vertikalposition (weiße Quadrate, untere Achse) bzw. der Zeit (schwarze Quadrate, obere Achse) und eine an die erste Steigung angefittete Sinusfunktion (Linie). 


\subsection{Oberflächenschwingungen}

Durch die Blasenpulsation können Oberflächenmoden angeregt werden [39, 33], die zur Fragmentation der Blase oder zur Abspaltung von Mikroblasen führen können (siehe Abschnitt 2.4). Dabei führen die Blase bzw. die Blasenfragmente meist sehr plötzliche, irreguläre Translationsbewegungen aus [24]. Über den genauen Mechanismus ist wegen der kurzen Zeitskalen (die Prozesse spielen sich in wenigen Schwingungsperioden ab) bisher jedoch wenig bekannt. In diesem Abschnitt wird die Abspaltung und Rekombination von Mikroblasen mit Hilfe von Hochgeschwindigkeitskinematographie untersucht. Dazu wird die Dynamik einer im zentralen Schalldruckbauch der $(2,1,1)$-Mode eines Plexiglasquaders gehaltenen Luftblase durch das Fernmikroskop phasenstarr mit einer Bildwiederholrate von $2.25 \mathrm{kHz}$ digital aufgezeichnet. Bei einer Anregungsfrequenz von $32 \mathrm{kHz}$ entspricht dies einer zeitlichen Auflösung von $15 \pm 1$ Schwingungsperioden. Der optische Aufbau besitzt eine räumliche Auflösung von $1.82 \mu \mathrm{m} /$ pixel. Um hohe Anwachsraten der Blase zu verhindern (siehe Abschnitt 2.3), wurde das Wasser leicht entgast $\left(c / c_{0} \approx 50 \%\right)$. In Abbildung 5.15 ist jeweils die Trajektorie über 43 Millisekunden einer oberflächenstabilen Blase (schwarze Punkte, $\left.R_{\text {max }}=40 \mu \mathrm{m}, p_{a}=70 \mathrm{kPa}, R_{0} \approx 26 \mu \mathrm{m}\right)$ und einer oberflächeninstabilen Blase (weiße Punkte, $R_{\max }=36 \mu \mathrm{m}, p_{a}=53 \mathrm{kPa}, R_{0} \approx 27 \mu \mathrm{m}$ ) abgebildet. Die höhere Schalldruckamplitude bei der oberflächenstabilen Blase hat ihre Ursache in einer etwas stärkeren Entgasung. Der Ursprung wurde in den jeweiligen geometrischen Schwerpunkt der Trajektorie gelegt. Der Ruheradius wurde aus Schalldruckamplitude und Maximalradius abgeschätzt.

Der Aktionsradius beider Blasen liegt innerhalb ihres Ruheradius. Die Bewegung der oberflächeninstabilen Blase ist jedoch sehr viel unsteter, was sich anhand der sehr unterschiedlichen Abstände der zeitlich äquidistanten Blasenpositionen zeigt. In Abbildung 5.15 ist die Verteilung der Geschwindigkeitsbeträge dargestellt. Während die oberflächenstabile Blase eine langsame Drift mit einem Häufigkeitsmaximum von etwa $1.5 \mathrm{~mm} / \mathrm{s}$ und einer maximalen Geschwindigkeit von knapp unter $10 \mathrm{~mm} / \mathrm{s}$ ausführt, liegt das Häufigkeitsmaximum der oberflächeninstabilen Blase nahe bei Null. Vereinzelt bewegt sie sich mit hohen Geschwindigkeiten bis zu $33 \mathrm{~mm} / \mathrm{s}$. Die Durchschnittsgeschwindigkeit ergibt sich für die stabile Blase zu $<v>_{t}=(3.1 \pm 1.8) \mathrm{mm} / \mathrm{s}$, für die instabile Blase $\mathrm{zu}\left\langle v>_{t}=(4.3 \pm 6.8) \mathrm{mm} / \mathrm{s}\right.$. 

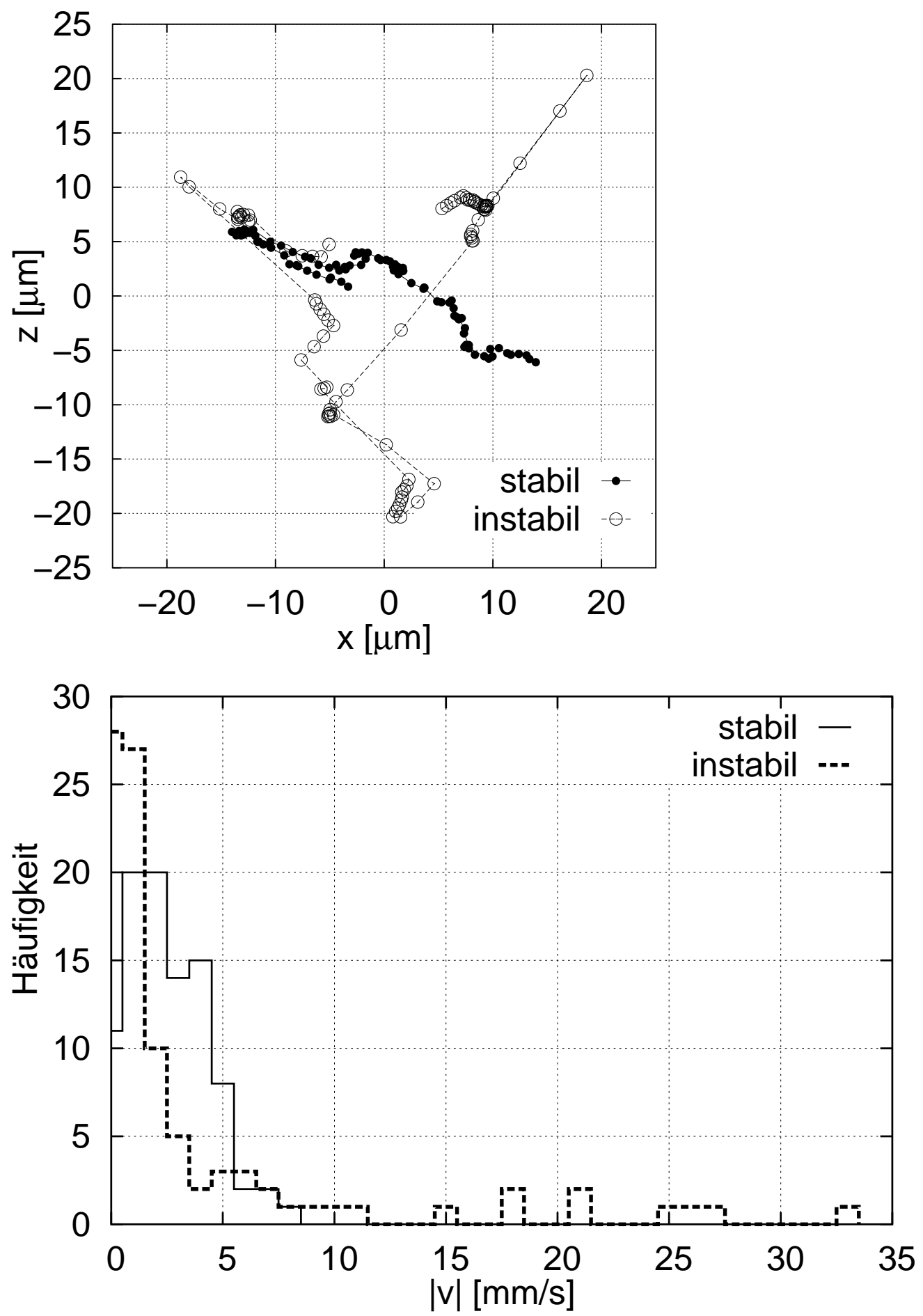

Abbildung 5.15: Trajektorie einer stabilen Blase (schwarze Punkte, $R_{\text {max }}=40 \mu \mathrm{m}, p_{a}=70 \mathrm{kPa}, R_{0} \approx 26 \mu \mathrm{m}$ ) und instabilen Blase (weiße Punkte, $\left.R_{\max }=36 \mu \mathrm{m}, p_{a}=53 \mathrm{kPa}, R_{0} \approx 27 \mu \mathrm{m}\right)$. Der Ursprung kennzeichnet den geometrischen Schwerpunkt der Trajektorien. 
Eine Auswertung der Filmsequenz der instabilen Blase zeigt unterschiedliches Abspaltungsverhalten. Zur Heraushebung der Blasenform werden die Bilder gefiltert und binarisiert. In Abbildung 5.16 - 5.18 sind die erkennbaren Abspaltungsereignisse aus einer Sequenz von 99 ms (247 Einzelbilder) dargestellt.

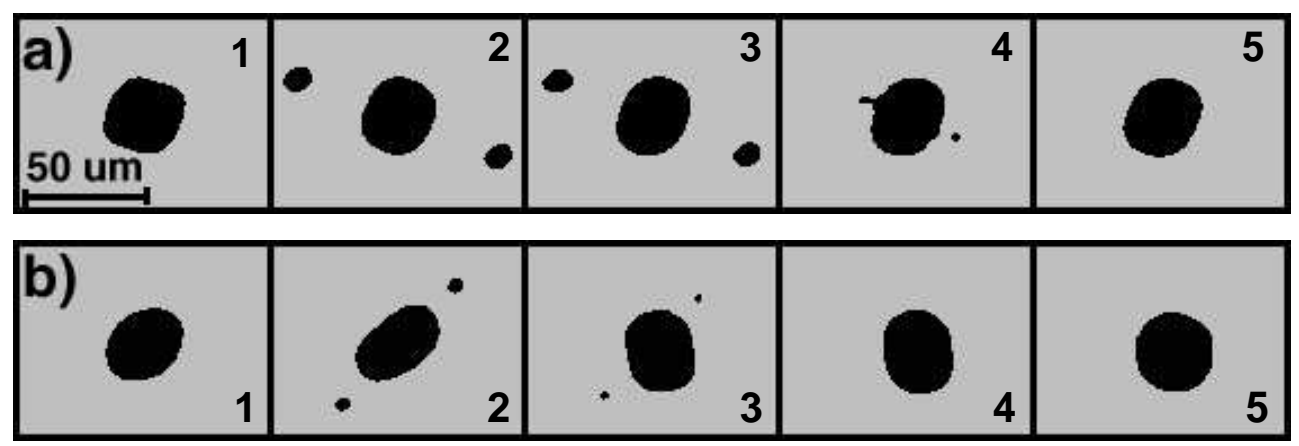

Abbildung 5.16: Symmetrische Abspaltung von zwei Mikroblasen durch Oberflächeninstabilitäten. Bildabstand $0.44 \mathrm{~ms}$.

Abbildung 5.16 zeigt die simultane Abspaltung von zwei Mikroblasen. Die Abspaltung erfolgt in diesem Fall symmetrisch. In Abbildung (a) ist die nahezu unveränderte Position der Mikroblasen in Bild 2 und 3 bemerkenswert, in Bild 4 hingegen sind die Blasen bereits kurz vor der Rekombination. In Abbildung (b) ist die $\mathrm{n}=2$ Oberfächenmode der Blase in Richtung der Abspaltung gut zu sehen. Abbildung 5.17 zeigt zwei Abspaltungsereignisse jeweils einer Mikroblase. Die Form der Blase beim ersten Auftauchen der Mikroblase (Bild 2 und insbesondere Bild 15) deuten auf eine Beteiligung der $n=3$ Mode hin. Die erste Mikroblase erreicht etwa $0.5 \mathrm{~ms}$ nach ihrem Auftauchen maximale Entfernung von der Hauptblase, um sich dann nach etwa $4 \mathrm{~ms}$ (Bild 9) wieder sichtbar zu nähern. Kurz nach der Rekombination wird eine Mikroblase in nahezu entgegengesetzter Richtung abgespalten. Die Vorzugsrichtung der Störung scheint hier durch den Impuls der zurückkehrenden Mikroblase gegeben zu sein. Auch hier nimmt die Mikroblase nach etwa $1 \mathrm{~ms}$ ihres Erscheinens eine Position ein, in der sie etwa $1.5 \mathrm{~ms}$ verharrt. Im Gegensatz zur ersten Mikroblase entfernt sich dann jedoch noch ein weiteres Mal und vereinigt sich nicht mit der Hauptblase. Die Mikroblase verbleibt während der gesamten weiteren Aufnahme (etwa 25 ms) auf dieser Position. Die abnehmende Sichtbarkeit in späteren Bildern deutet auf ein langsames Wegdiffundieren der Mikroblase hin. Erstaunlich ist, dass diese Blase nicht durch die sekundäre Bjerkneskraft von der Hauptblase angezogen wird. 


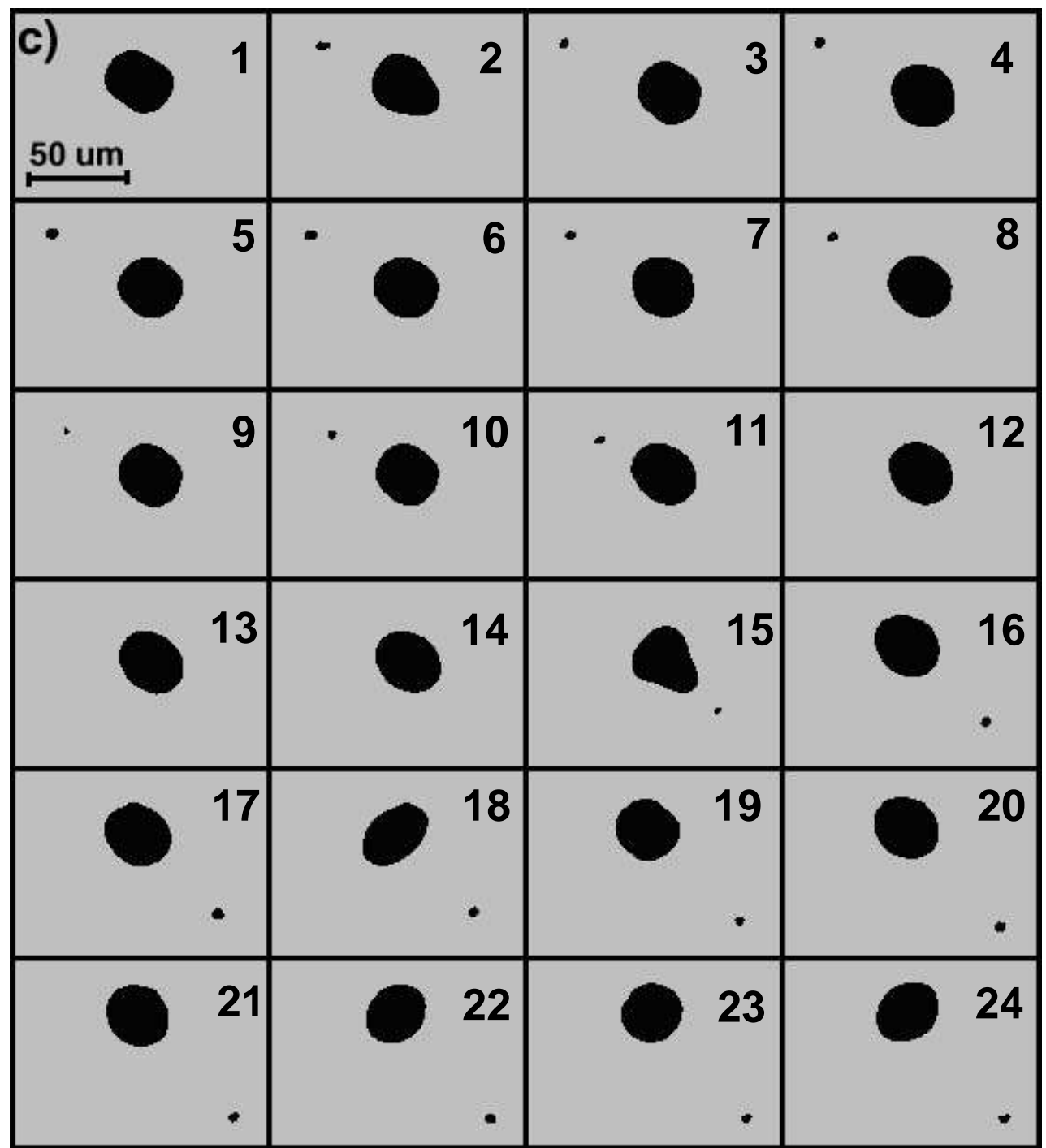

Abbildung 5.17: Abspaltung einer Mikroblase durch Oberflächeninstabilitäten. Bildabstand $0.44 \mathrm{~ms}$.

Möglicherweise spielen hier durch die Oberflächenschwingungen induzierte Mikroströmungen eine Rolle [39]. Allerdings schwingt die Hauptblase im Verlauf der Messung immer wieder nahezu sphärisch. Deshalb liegt die Vermutung nahe, dass die Mikroblase zu schwach pulsiert, um noch eine hinreichende sekundäre Bjerkneskraft aufzubauen. 


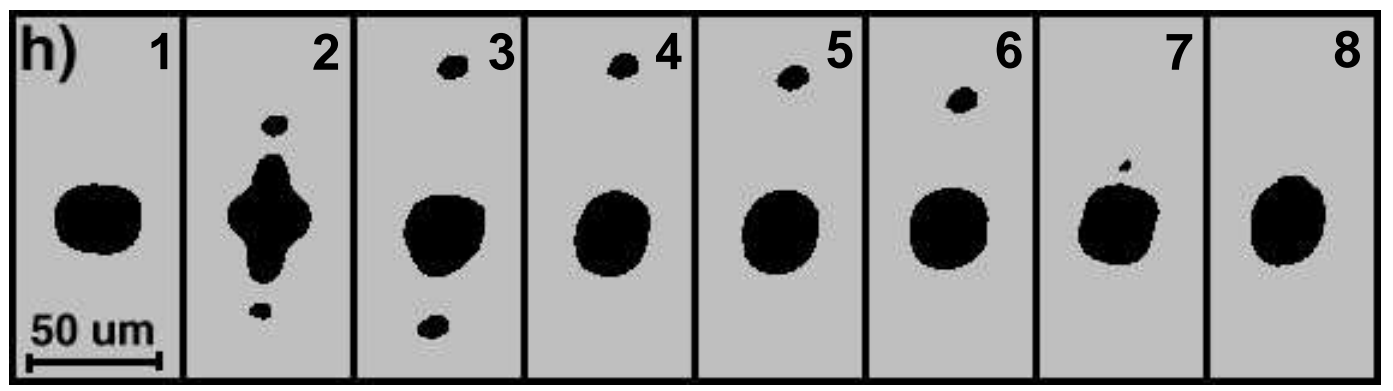

Abbildung 5.18: Unsymmetrische Abspaltung von Mikroblasen durch Oberflächeninstabilitäten. Bildabstand $0.44 \mathrm{~ms}$.

Abbildung 5.18 zeigt die simultane, unsymmetrische Abspaltung von zwei Mikroblasen. Gut sichtbar ist die $\mathrm{n}=4$ Mode der Hauptblase bei der Abspaltung (Bild 2), die zunächst symmetrisch zu erfolgen scheint. Im darauffolgenden Bild jedoch ist die Hauptblase deutlich nach unten ausgelenkt und in Richtung der unteren, näheren Blase deformiert. Im weiteren Verlauf rekombiniert zuerst die untere Mikroblase (0.8 ms), nach $2 \mathrm{~ms}$ die obere Mikroblase, wobei die Hauptblase sich wieder nach oben bewegt und etwa in der Ausgangsposition endet.

Ein Simulation mit einem Partikelmodell ergibt eine gute qualitative Übereinstimmung mit den experimentellen Ergebnissen, zeigt aber eine zu starke Beschleunigung der Hauptblase [76, 53]. Der Vergleich zwischen Experiment und Simulation ist in Abbildung 5.19 dargestellt. Die Abweichungen können in einer Fehleinschätzung der sekundären Bjerkneskraft begründet sein. Möglich ist aber auch hier ein nicht zu vernachlässigender Einfluss der Oberflächenschwingung auf die Bewegung der Blase.

Insgesamt zeigt sich ein deutlicher Zusammenhang zwischen dem Einsetzen von Oberflächenmoden und der Abspaltung von Mikroblasen. Das häufige Auftreten von zwei gegenüberliegenden Mikroblasen deutet auf eine dominierende Rolle der $\mathrm{n}=2$ Mode hin, die mit zusätzlichen höheren Moden gekoppelt sein kann. Aufgrund ihrer geringeren virtuellen Masse werden die Mikroblasen durch die - bei kleinen Abständen symmetrische - sekundäre Bjerkneskraft stärker beschleunigt als die Hauptblase (siehe Abschnitt 2.2). Dies zeigt sich im Vergleich der Geschwindigkeitsverteilung von Hauptblase und den Mikroblasen. Diese ist, normiert auf die Häufigkeit bei $|v|=(0-5) \mathrm{mm} / \mathrm{s}$, in Abbildung 5.20 dargestellt. Die Geschwindigkeit der rekombinierenden Blasen wurde aus der Position die Blasenwand im Ruheradius bestimmt, dies gibt eine untere Abschätzung der Geschwindigkeit. Die Hauptblase erreicht eine Maximalgeschwindigkeit von $75 \mathrm{~mm} / \mathrm{s}$. Bei Mikroblasen können vereinzelt Geschwindigkeiten bis $200 \mathrm{~mm} / \mathrm{s}$ beobachtet werden. 


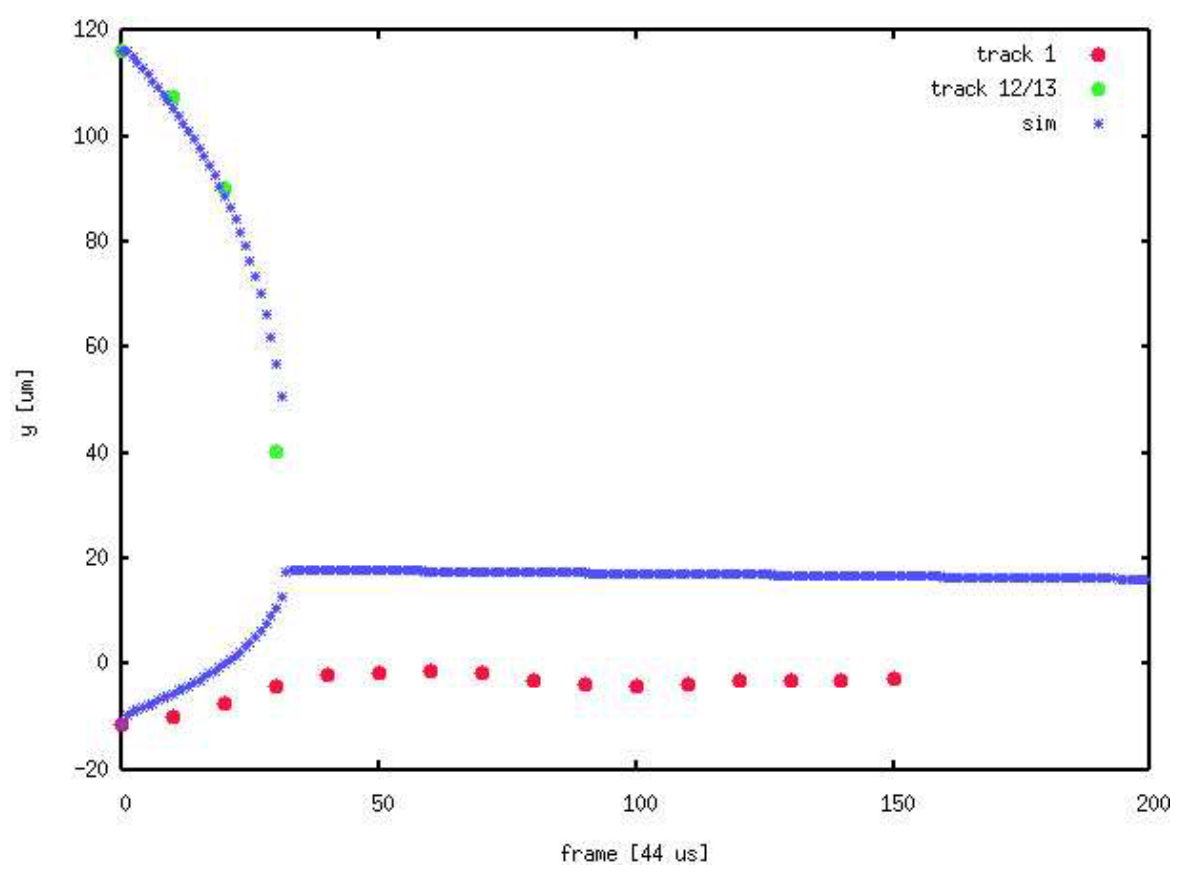

Abbildung 5.19: Simulation der Rekombination der Haupt- und Mikroblase aus Sequenz 5.18. Die roten Punkte bezeichnen die vertikale Position der Hauptblase, die grünen Punkte die der oberen Mikroblase. Die blauen Kreuze zeigen die aus den Anfangswerten berechneten Trajektorien. Simulation und Abbildung von P. Koch.

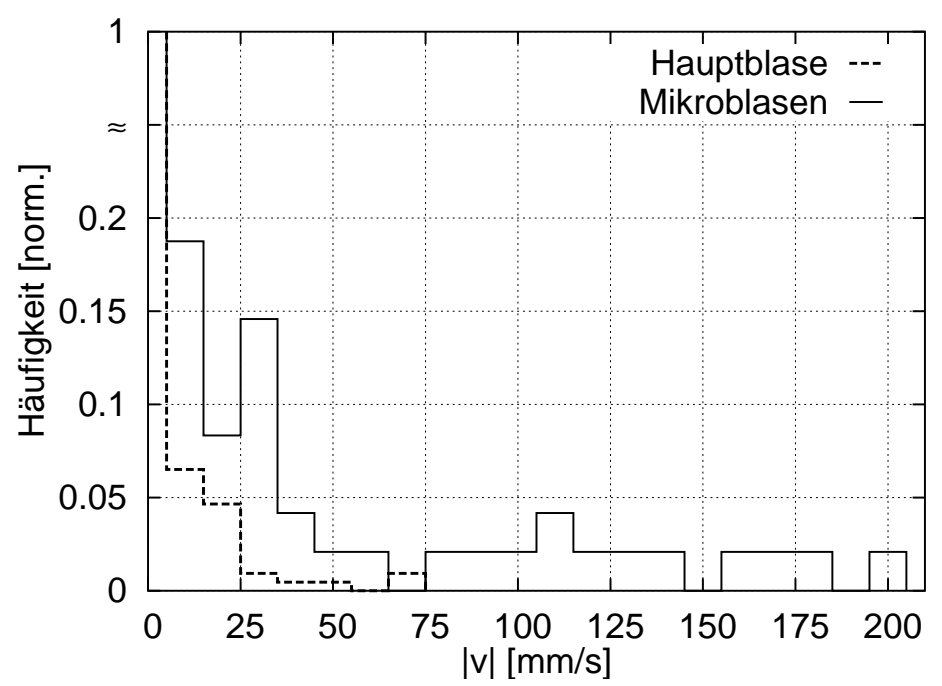

Abbildung 5.20: Relative Häufigkeiten der Geschwindigkeit $|v|$ der Hauptblase (gestrichelt) und der abgespaltenen Mikroblasen (durchgezogen). 


\subsection{SBSL in luftgesättigtem Wasser}

Entgegen der allgemein verbreiteten Meinung, Einzelblasensonolumineszenz (SBSL) sei nur in mindestens zu $50 \%$ entgastem Wasser realisierbar [44, 33, 14, 43], können auch in luftgesättigtem Wasser bei Zimmertemperatur und Atmosphärendruck sowohl stabile als auch periodisch instabile lichtemittierende Blasen erzeugt werden [56].

\subsubsection{Durchführung}

Als akustischer Resonator wird der Glasquader in der Grundmode verwendet (siehe A.1). Dieser Resonator zeichnet sich vor allem durch die Abwesenheit von Störstellen aus, die als Blasenquellen dienen können. Wesentlich für die Erzeugung von stabilen Einzelblasen war die Injektion im Schalldruckbereich der SBSL oder weniger $\mathrm{kPa}$ darunter $\left(p_{a}=100-120 \mathrm{kPa}\right)$. Injektion einer Blase bei niedrigeren Schalldruckamplituden führt zu einer sehr großen, oberflächeninstabilen Blase, bei zu hohem Schalldruck verschwindet die injizierte Blase vor Erreichen des Schalldruckbauches. Die Blasendynamik wurde mit der digitale Videokamera (PULNiX TM-6701 AN) durch das Fernmikroskop (Questar QM 100) aufgezeichnet. Dazu wurde die Phasendifferenz zwischen Anregung und Mikrosekunden-Blitz bei jeder Aufnahme vergrößert. Zum Vergleich wurde eine SBSL-Blase in leicht entgastem Wasser $\left(c_{\infty} / c_{0}=0.42, c_{\infty}\right)$ mit dem gleichen Messverfahren untersucht. Zur Ermittlung der Radius-Zeit-Kurven werden die Einzelbilder zu einer Streakaufnahme zusammengestellt, wobei Variationen in der Blasenposition korrigiert werden. Zum Vergleich wird die Lösung der Gilmore-Gleichung mit variablem Polytropenexponenten an den Gleichgewichtsradius und den Maximalradius angefittet.

Zur Bestimmung des Lumineszenzverhaltens wird die Lichtemission von jeweils 50 Lichtblitzen mit einem fokussierten Photomultiplier (Hamamatsu R5600U) bei unterschiedlichem Gasgehalt und unterschiedlicher Anregungsamplitude mit einem Oszilloskop aufgezeichnet.

Um die Stabilität der SBSL in luftgesättigtem Wasser genauer zu bestimmen, werden simultan die Lichtemission und die Dynamik der Blase bestimmt. Dazu wird die Blasengröße und -position alle hundert Perioden stroboskopisch aufgezeichnet (HiSIS 2002 + Questar QM 100). Das PMT-Signal konnte jede 1500ste Periode ausgelesen werden, der limitierende Faktor ist hier die Übertragungsrate des verwendeten Oszilloskops (Tektronix TDS 748). 


\subsubsection{Ergebnisse}

Abbildung 5.21 gibt die Radius-Zeit-Kurven von Einzelblasen im SBSL - Bereich in gesättigtem $(\mathrm{a}, \mathrm{b})$ sowie in leicht entgastem Wasser $(\mathrm{c}, \mathrm{d})$ an jeweils der unteren und oberen Stabilitätsgrenze bezüglich der Schalldruckamplitude wieder. An beiden Grenzen setzen Oberflächeninstabilitäten ein.
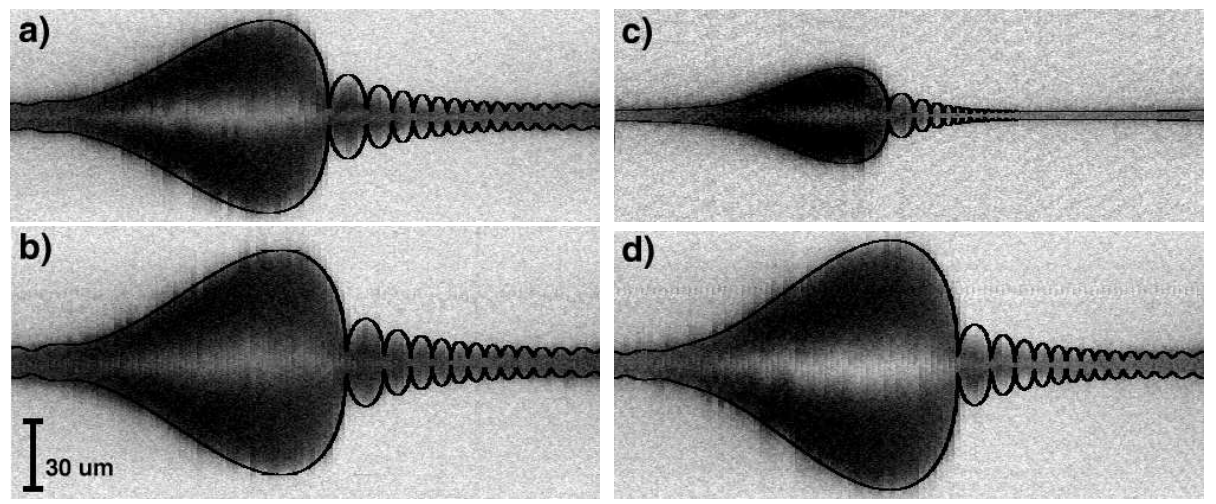

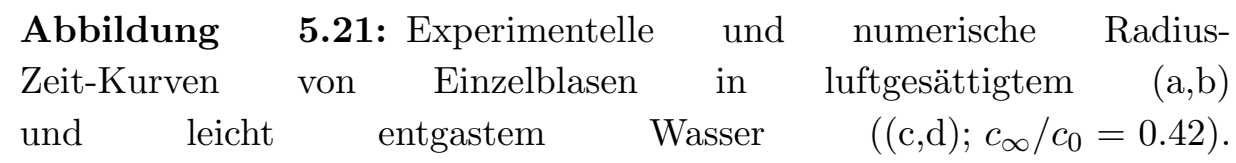

a) Untere Stabilitätsgrenze, $R_{0}: 5.5 \mu \mathrm{m}, R_{\max }: 42.0 \mu \mathrm{m}, p_{a}: 125 \mathrm{kPa}$.

b) Obere Stabilitätsgrenze, $R_{0}: 6.0 \mu \mathrm{m}, R_{\max }: 49.7 \mu \mathrm{m}, p_{a}: 130 \mathrm{kPa}$.

c) Untere Stabilitätsgrenze, $R_{0}: 2.4 \mu \mathrm{m}, R_{\max }: 21.4 \mu \mathrm{m}, p_{a}: 121 \mathrm{kPa}$.

d) Obere Stabilitätsgrenze, $R_{0}: 5.5 \mu \mathrm{m}, R_{\max }: 55.5 \mu \mathrm{m}, p_{a}: 135 \mathrm{kPa}$.

Der Gleichgewichtsradius liegt bei luftgesättigtem Wasser im stabilen Bereich bei $(5.5-6.0 \pm 0.5) \mu \mathrm{m}$, das Expansionsverhältnis liegt etwa bei $R_{\max } / R_{0}=8$. Der Gleichgewichtsradius an der oberen Stabilitätgrenze in leicht entgastem Wassser (d) von $R_{0}=6 \mu \mathrm{m}$ korrespondiert mit denen in luftgesättigtem Wasser gemessenen Radien. An der unteren Stabilitätsgrenze (c) ist der Radius deutlich kleiner. Als weitere Beobachtung ist anzumerken, dass die Blase in gesättigtem Wasser Translationen in einem Aktionsradius von etwa $10 \mu \mathrm{m}$ durchführt, während die Blasenposition in leicht entgastem Wasser mit einer Unsicherheit von etwa $1 \mu \mathrm{m}$ konstant ist.

Die Lumineszenz ist mit adaptiertem Auge auch in schwach beleuchteten Räumen sichtbar. In Abbildung 5.22 ist die Amplitude sowie der Zeitpunkt des Lumineszenzpulses - und damit indirekt des Blasenkollaps - wiedergegeben. Das Ansteigen und spätere Abfallen der Lichtintensität bei Erhöhung der Anregungsamplitude ist analog zum Verhalten von SBSL bei niedrigem Gasgehalt [6, 33, 51, 55]. 


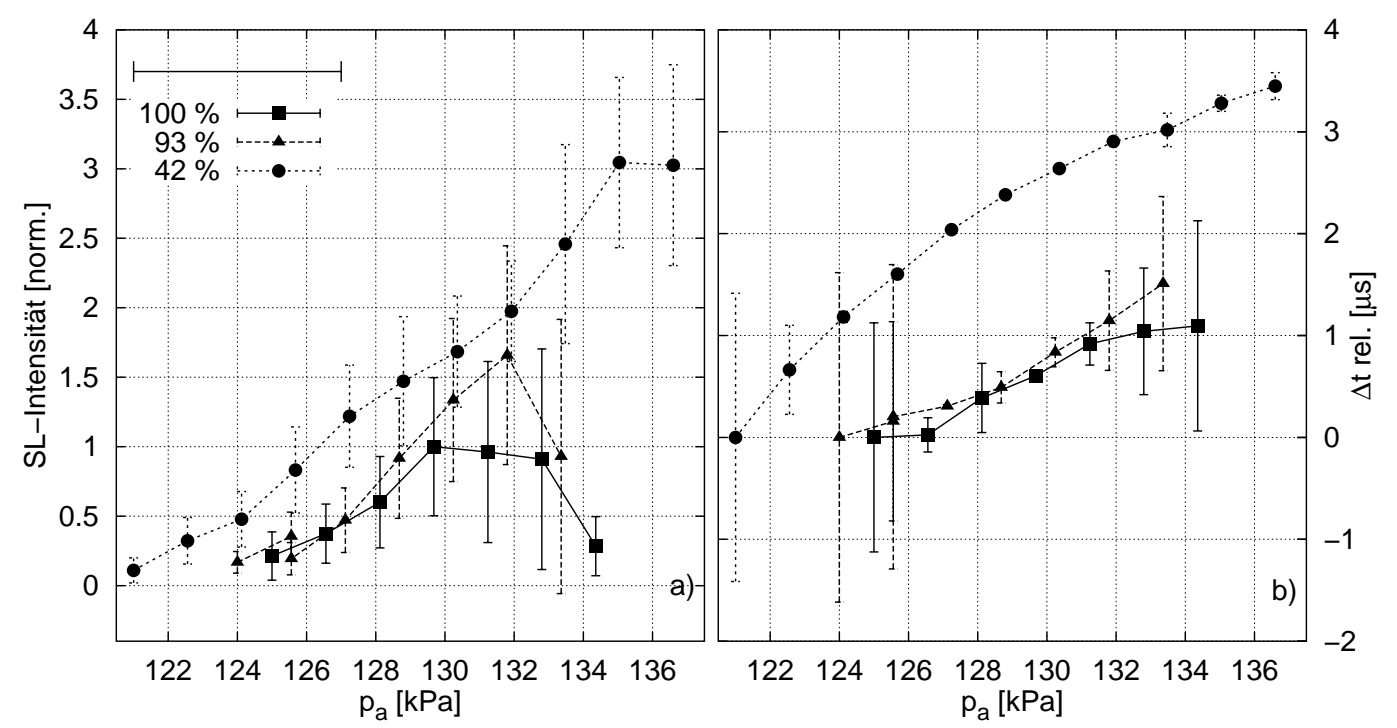

Abbildung 5.22: Lichtintensität (a) und Zeitpunkt $\Delta t_{\text {rel }}$ (b) der Lichtemission. Die Lichtintensität ist auf die maximale Intensität in gesättigtem Wasser normalisiert, der Zeitpunkt relativ zum Lichtblitz bei jeweils niedrigster Schalldruckamplitude bei konstantem Gasgehalt.

In entgastem Wasser lassen sich deutlich höhere Lichtemissionen erzeugen, dies aber vor allem durch die Verschiebung des Stabilitätsbereiches zu höheren Schalldruckamplituden. Da die Schwankungen in der Lichtintensität generell groß sind und eher von der mittleren Lichtintensität als der Stabilität der Sonolumineszenz abhängen, ist die Varianz im Zeitpunkt der Lichtemission ein besserer Indikator für die Stabilität [6]. So zeigen sich große temporale Schwankungen beim Einsetzen der SBSL sowie bei hohen Anregungsamplituden. Während die Varianz der Lichtintensität bei gesättigtem Wasser noch einmal kurz vor der Zerstörungsgrenze abnimmt, zeigt die zunehmende Varianz in der relativen Phase der Lichtemission, dass die Stabilität mit steigendem Gasgehalt abnimmt. Zudem verschiebt sich der Kollaps bei Erhöhung der Schalldruckamplitude mit zunehmendem Gasgehalt weniger stark.

Simulationen von Blasenschwingungen unter Versuchsbedingungen, die die Keller - Miksis - Gleichung unter Berücksichtigung von Diffusion und Oberflächenschwingungen lösen, zeigen für einen Gasgehalt von $1 \%$ der Sättigungskonzentration von Luft in einem schmalen Schalldruckbereich von $120-135 \mathrm{kPa}$ stabile Blasen [1, 58, 85, 95](Simulationen durchgeführt von R. Mettin). Dieser Anteil entspricht der Edelgasmenge in Luft, und ist unter der Annahme der Dissoziationshypothese, dass bei heftig kollabierenden Blasen reaktive Gase zu wasserlöslichen Produkten reagieren und in der Blase lediglich Edelgas verbleibt 


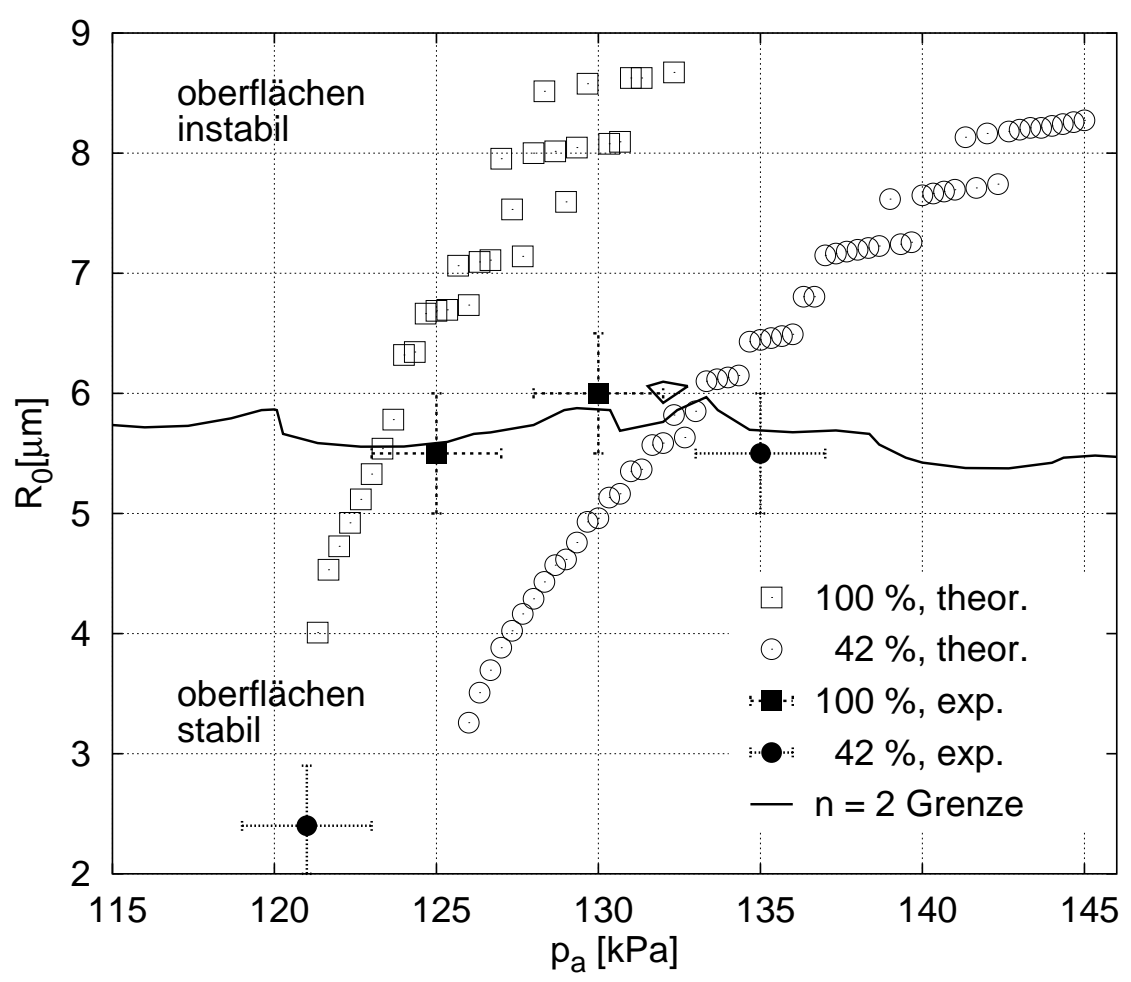

Abbildung 5.23: Experimentelle (schwarze Symbole) and numerisch bestimmte (weiße Symbole) Gleichgewichtsradien in Abhängigkeit von der Schalldruckamplitude in luftgesättigtem (Quadrate) and leicht entgastem Wasser (Kreise). Die durchgezogene Linie gibt die Grenze der parametrischen Stabilität der n=2 Oberflächenmode wieder.

[68], der am Diffusionsprozess beteiligte Gasanteil. Abbildung 5.23 zeigt die experimentellen Ergebnisse aus $5.21 \mathrm{im}$ Vergleich mit den Simulationsergebnissen.

Der Bereich der stabilen Blasenschwingungen in gesättigtem Wasser, der bei $p_{a}=121-124 \mathrm{kPa}$ liegt, kann experimentell nicht erreicht werden. Die in diesem Bereich beobachtete Fragmentation der Blase kann durch unvollständige Dissoziation der reaktiven Gase erklärt werden [97]. Ist der Kollaps zu schwach, verbleibt ein höherer Gasanteil in der Blase, der Gleichgewichtsradius ist größer und die Blase ist oberflächeninstabil. Die Simulation des Bereichs unvollständiger Dissoziation, in dem der effektive Gasgehalt zwischen Luftgehalt und Edelgasmenge im Wasser liegt, erfordert die Berücksichtigung der chemischen Reaktionen und ist in diesem Modell nicht berücksichtigt. Die experimentell gefundenen Blasen liegen im Schalldruckbereich, in dem das Modell instabile Pulsationen vorhersagt. Die gemessenen Radien sind deutlich kleiner als die numerisch bestimmten und 


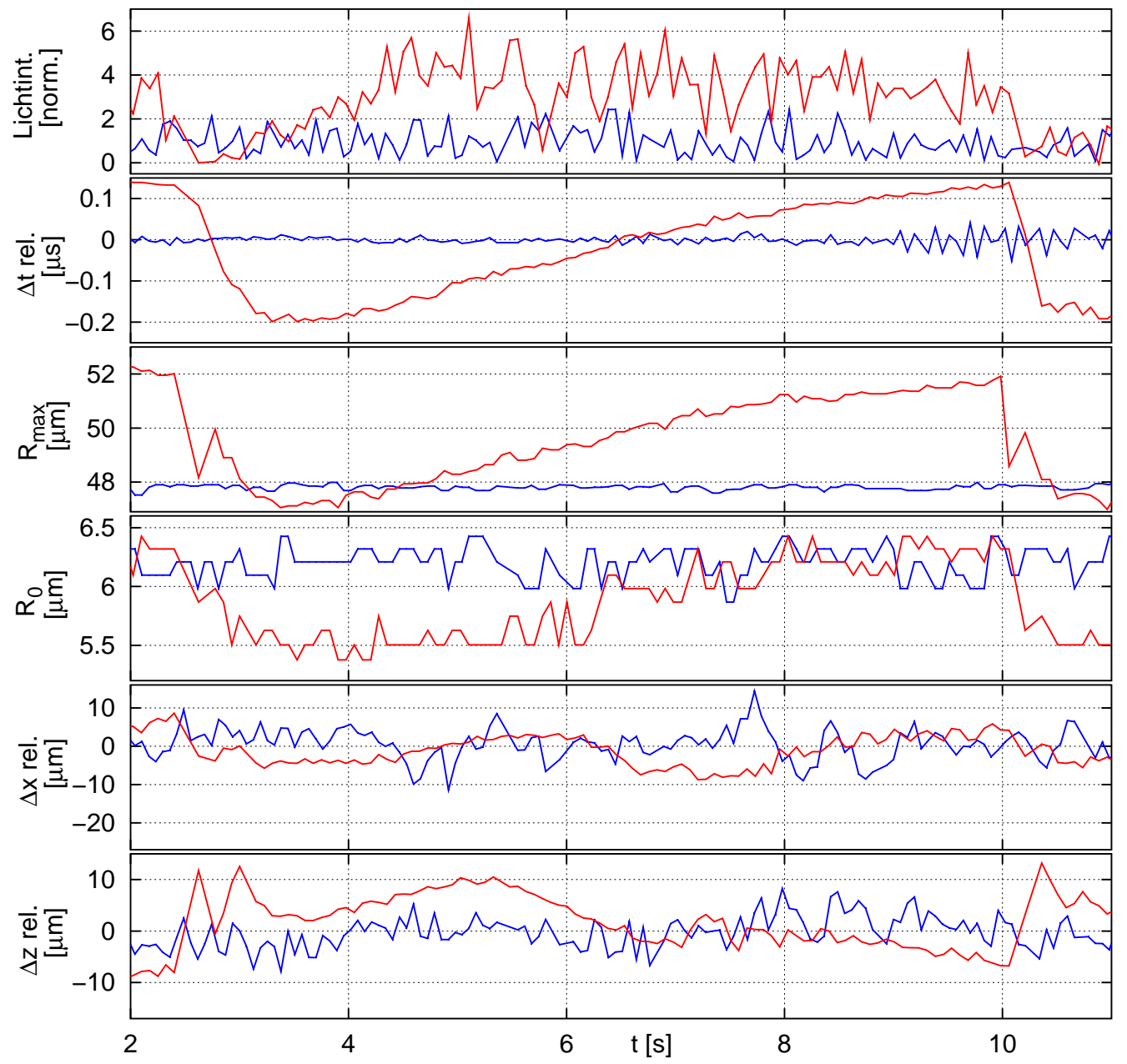

Abbildung 5.24: Zeitverlauf von Lichtemission, $R_{\max }, R_{0}$ sowie die Translationsdynamik einer stabilen (blau) und einer zyklischen SBSLBlase (rot) in gesättigtem Wasser.

liegen an der Stabilitätsgrenze der $\mathrm{n}=2$ Mode. Diese ist in der Grafik durch eine schwarze Linie gekennzeichnet. Dies gilt auch für die SBSL Blase in leicht entgastem Wasser an der oberen Stabilitätsgrenze. In Bereichen, in denen der Gleichgewichtsradius oberhalb des Bereiches formstabiler Pulsationen liegt, erwartet man das Phänomen der zyklischen SBSL (recycling SBSL) [6, 13, 44]. Bei konstanter Anregung wächst die Blase, um dann plötzlich, nach Einsetzen von Oberflächeninstabilitäten, ihren Radius zu verkleinern. Dies geht einher mit einer steten Zunahme der Lichtintensität, die dann plötzlich einbricht, um den Zyklus erneut zu durchlaufen.

Abbildung 5.24 zeigt das Verhalten zweier SBSL-Blasen in gesättigtem Wasser. 
Deutlich ist der simultane Verlauf von Minimal-, Maximalradius und Zeitpunkt des Kollaps zu sehen. Während bei der blau markierten, stabilen SBSL diese Werte konstant bleiben, ist bei der rot markierten Blase ein zyklisches Verhalten zu beobachten. Einer schnellen Verringerung der Werte folgt ein langsameres Anwachsen über mehrere Sekunden, um dann wieder auf vergleichsweise kurzen Zeitskalen abzunehmen. Die Einbrüche sind auch in der stark verrauschten Lichtintensität zu beobachten. Die Lichtintensität steigt jedoch bereits, während der Kollapszeitpunkt und die Radien noch fallen, und erreicht bereits nach etwa 2 Sekunden ein Intensitätsmaximum. Interessant ist auch die Translationsdynamik. Die stabile Blase weist eine stärkere Fluktuation der Blasenposition auf. Insgesamt sind die Auslenkungen von der mittleren Position vergleichbar, bei der instabilen Blase ist die Bewegung jedoch stetiger. In vertikaler Richtung wird die zyklische Blase erwartungsgemäß mit steigendem Radius nach unten in den Schalldruckbauch gezogen. Auch in dieser Richtung ist die Bewegung der stabilen Blase weniger stetig.

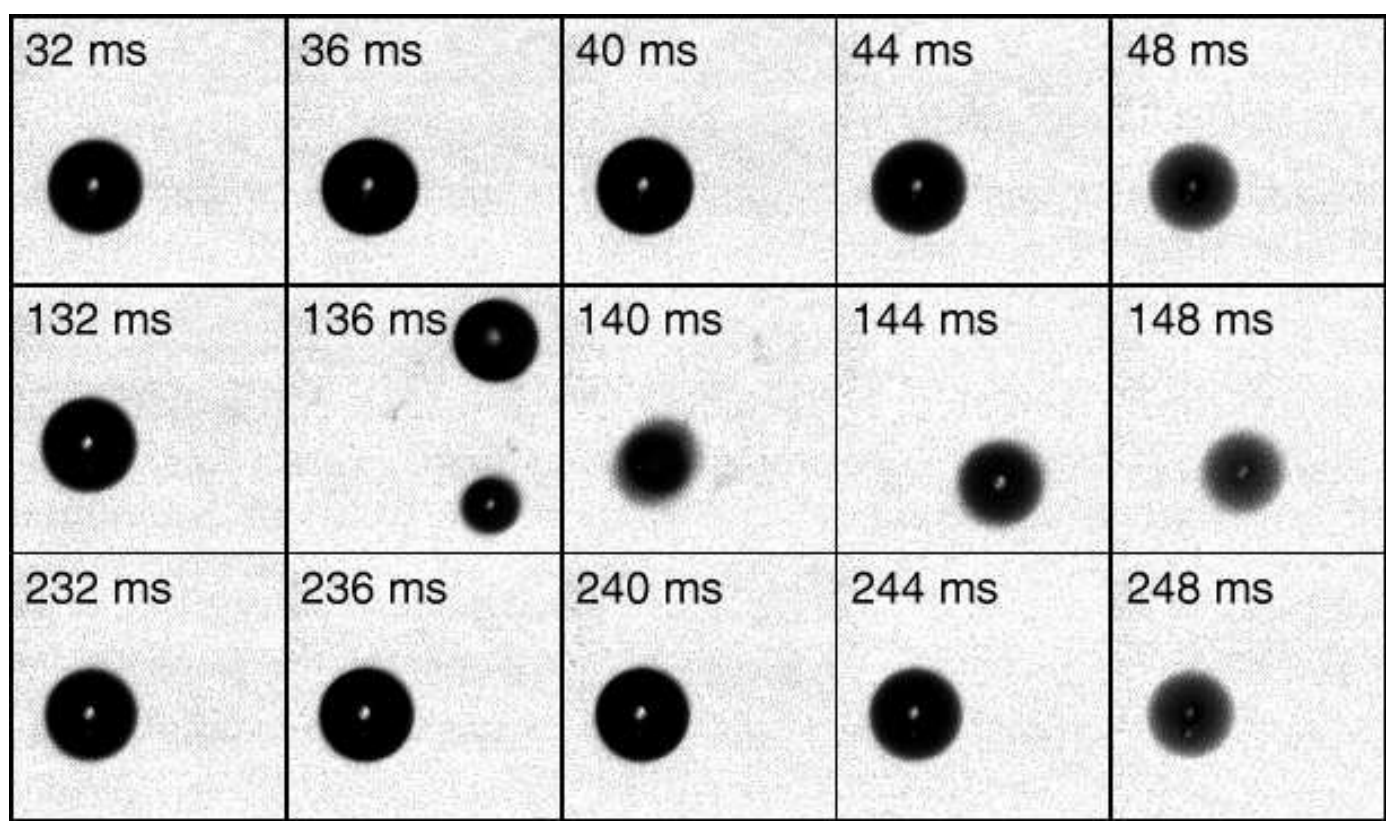

Abbildung 5.25: Fragmentation und Rekombination der zyklischen SBSL-Blase aus Abbildung $5.24\left(t_{0}=10 \mathrm{~s}\right)$. Die Phasendifferenz zwischen den horizontal angeordneten Bildern beträgt $\Delta \phi=1 / 25$ T. Die Bilder einer Spalte sind bei gleicher Phasenlage aufgenommen.

Die Vermutung, der plötzliche Einbruch des Radius werde durch Oberflächeninstabilitäten verursacht, wird durch die Filmaufnahmen bestätigt. Abbildung 5.25 zeigt die Blasendynamik im Bereich des zweiten Einbruchs in Abbildung $5.24\left(t_{0}\right.$ $=10 \mathrm{~s}$ ). Eine horizontale Bildsequenz entspricht einem Ausschnitt aus einem Pha- 
senzyklus, der jeweils 25 Bilder umfasst. Bilder innerhalb einer Spalte sind also phasenstarr. Beobachtet wird die Fragmentation der SBSL-Blase in (mindestens) zwei Teilblasen. Dies deutet auf die Dominanz der $\mathrm{n}=2$ Mode hin (siehe Abschnitt 5.3). Mikroblasenabspaltung konnte nicht beobachtet werden, die starke horizontale Auslenkung der Fragmente im Vergleich zur ursprünglichen Blasenposition deutet jedoch auf mögliche Fragmente unterhalb der Auflösungsgrenze hin. Bereits im Bild nach dem Fragmentationsereignis (100 Perioden später) rekombiniert die Blase und springt dabei in ihre ursprüngliche Position. Die Unschärfe des Blasenradius lässt $n=2$ Oberflächenschwingungen vermuten. Anschließend ändert die Blase nochmals die Position, um dann langsam in die Ausgangsposition zurückzulaufen. Im nächsten Phasenzyklus ist bereits kaum ein Unterschied zum Verhalten vor der Fragmentation zu sehen. Es zeigt sich, dass der minimale Gleichgewichtsradius nicht instantan, sondern einige 100 Perioden nach der Fragmentation erreicht wird. Dies kann durch Dissoziationsprozesse von in die Blase gelangten, reaktiven Gasen verursacht sein [46]. Die Existenz von nach üblichen Kriterien stabiler SBSL in luftgesättigtem Wasser in einem Schalldruckbereich, der zyklische SBSL vermuten lässt, ist möglicherweise auf eine Art Selbstjustage der Blase auf den maximal stabilen Gleichgewichtsradius zurückzuführen. Liegen die Diffusionszeiten unterhalb der charakteristischen Zeitskala für das Anschwingen der Oberflächenmoden, so kann die Blase auf Forminstabiläten reagieren, bevor ein signifikanter Diffusionsprozess stattgefunden hat. Die vergleichsweise unsteten Positionsänderungen der ansonsten stabilen SBSL-Blase deuten auf die Abspaltung von Mikroblasen hin. Diese konnten im Experiment nicht beoachtet werden, müssen also entweder unter der Auflösungsgrenze von $0.5 \mu \mathrm{m}$ liegen oder eine sehr kurze Lebensdauer haben. 


\section{Kapitel 6}

\section{Die Clusterstruktur}

Die im Folgenden "Cluster"genannte Blasenstruktur taucht bei vergleichsweise hohen Schalldruckamplituden auf. Sie ist stabil, wenn die Schalldruckamplitude in Abwesenheit des Clusters über $190 \mathrm{kPa}$ beträgt. Der Cluster kann in einen blasenfreien Resonator injiziert werden, wobei sich die Schalldruckamplitude um bis zu 10 Prozent verringert und ein starker harmonischer Schallanteil in der Küvette generiert wird. Zusätzlich ist ein hörbares Rauschen zu vernehmen. Die Cluster sind nach der lumineszierenden Einzelblase bei steigender Schalldruckamplitude die nächste stationäre Struktur. Der Schalldruckbereich, in dem der Cluster existiert, schließt jedoch nicht direkt an den SBSL-Bereich an. Im Bereich von $p_{a}=140-190 \mathrm{kPa}$ kann keine Struktur induziert werden. Bei einer Verringerung der Schalldruckamplitude unter $p_{a}=190 \mathrm{kPa}$ verschwindet der Cluster plötzlich ohne nennenswerte Hysterese. Der Cluster ist innerhalb eines Translationsradius von etwa $1 \mathrm{~mm}$ ortsstabil.

\subsubsection{Durchführung}

Zur Bestimmung der Dynamik auf mittleren Zeitskalen werden Hochgeschwindigkeitsaufnahmen mit einem Bildabstand von etwa 11 Perioden der Anregungsfrequenz und einer Belichtungszeit von $5 \mu$ s angefertigt. Anschließend wird die Dynamik mithilfe von Ultrahochgeschwindigkeitskinematographie zeitlich höher aufgelöst. Das akustische Spektrum wird mit einem Hydrofon (Bruel \& Kjaer 8103) aufgenommen, das in einer oberen Ecke des Resonators nur etwa $1 \mathrm{~cm}$ (bis zum akustischen Zentrum) eingetaucht wurde, um seinen Einfluss so gering wie möglich zu halten. Es werden 1000 Einzelspektren aus jeweils 8192 Messwerten gebildet. Die Daten werden mit einem Hann-Fenster multipliziert und anschließend Fourier-transformiert. Um den Rauschanteil zu reduzieren, werden die Einzelspektren anschließend gemittelt. 


\subsubsection{Ergebnisse}

Abbildung 6.1 zeigt eine eine Filmsequenz mit einem Bildabstand von etwa 11 Perioden der Anregungsfrequenz und einer Belichtungszeit von $5 \mu$ s. Die Phase ist so gewählt, dass der Maximalradius sichtbar ist. Die Ruheradien können bei einer Auflösung von $4.5 \mu \mathrm{m} /$ pxl nicht aufgelöst werden, eine höhere räumliche Auflösung ist wegen der Translationsbewegung des Clusterschwerpunktes problematisch. Teilweise sind einzelne Fragmente zu sehen, oft scheint es sich um im Aufschwingen zusammengewachsene Fragmente zu handeln. Im Gegensatz zur oberflächeninstabilen Einzelblase (siehe Abschnitt 5.3) sind im Konglomerat einzelne sphärische Strukturen erkennbar. Eine sphärische Form des Clusters ist nicht erkennbar, sie ist unregelmäßig innerhalb eines Radius von ungefähr $R_{C}=0.2 \mathrm{~mm}$ verteilt. Die Dynamik innerhalb des Clusters ist zu schnell, als dass einzelne Fragmente verfolgt werden könnten. Die Anzahl der sichtbaren Fragmente in der gesamten Sequenz von 244 Einzelbildern liegt zwischen 1 und 15, die Maximalradien betragen 15 - $65 \mu \mathrm{m}$. Damit sind die Ruheradien in diesem Schalldruckbereich etwa $1 \mu \mathrm{m}$ groß, also nahe der dynamischen Blakeschwelle.

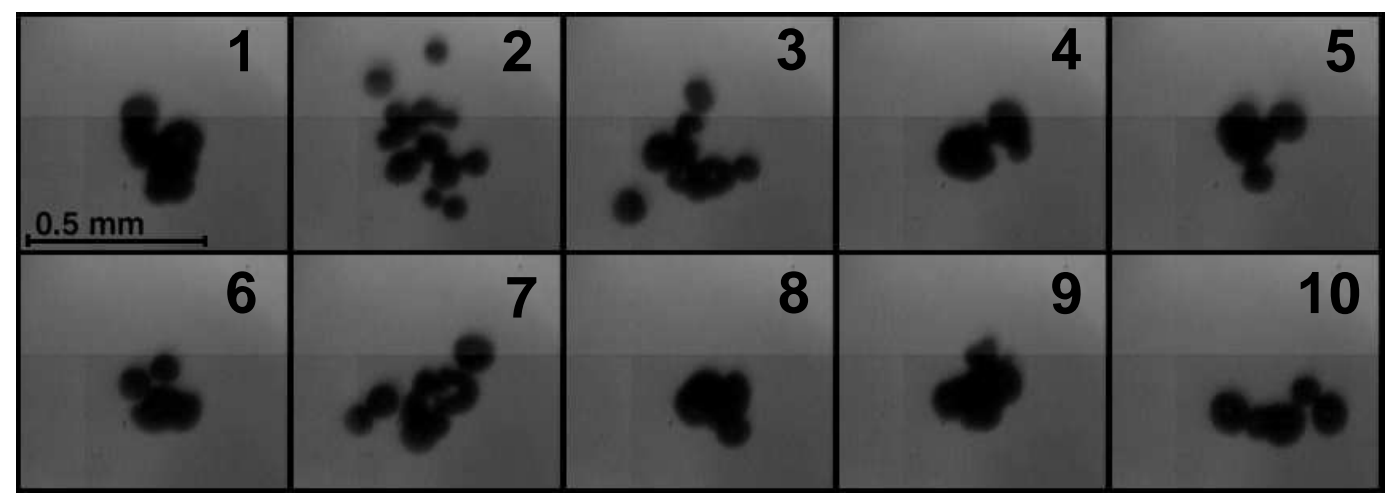

Abbildung 6.1: Clusterstruktur in der Phase maximalen Aufschwingens im zeitlichen Abstand von etwa 11 Perioden der Anregungsfrequenz. Belichtungszeit $5 \mu \mathrm{s}$.

Ultrahochgeschwindigkeitsaufnahmen zeigen, dass die Blasen bereits nach einer Periode nicht mehr zuzuordnen sind. Abbildung 6.2 zeigt den Cluster im Abstand von einer Periode. Abbildung 6.3 zeigt das Aufschwingen und Kollabieren des Clusters. Beim Aufschwingen berühren sich benachbarte Blasen, die teilweise beim Kollaps wieder vereinzeln. In der sichtbaren Phase der Blasenschwingung behält der Cluster seine Form bei. Der Bildabstand beträgt $t=T / 8$, auf den letzten drei Bildern ist keine Blase zu sehen, die Auflösung liegt bei etwa $7 \mu \mathrm{m} / \mathrm{pxl}$. Abbildung 6.4 zeigt die Blasendynamik zweier aufeinander folgender Perioden. 
Im Kollaps scheinen sich die Blasen wieder zu vereinigen, während sie in der Aufschwingphase bereits fragmentiert ist. Von einem Blasencluster zu reden, ist von daher problematisch, da es sich offensichtlich eher um eine beständig fragmentierte Einzelblase denn um einen Cluster längerlebiger Einzelblasen handelt. Da jedoch die Struktur im sichtbaren Bereich aus mehreren Blasen besteht, soll der Begriff hier beibehalten werden.

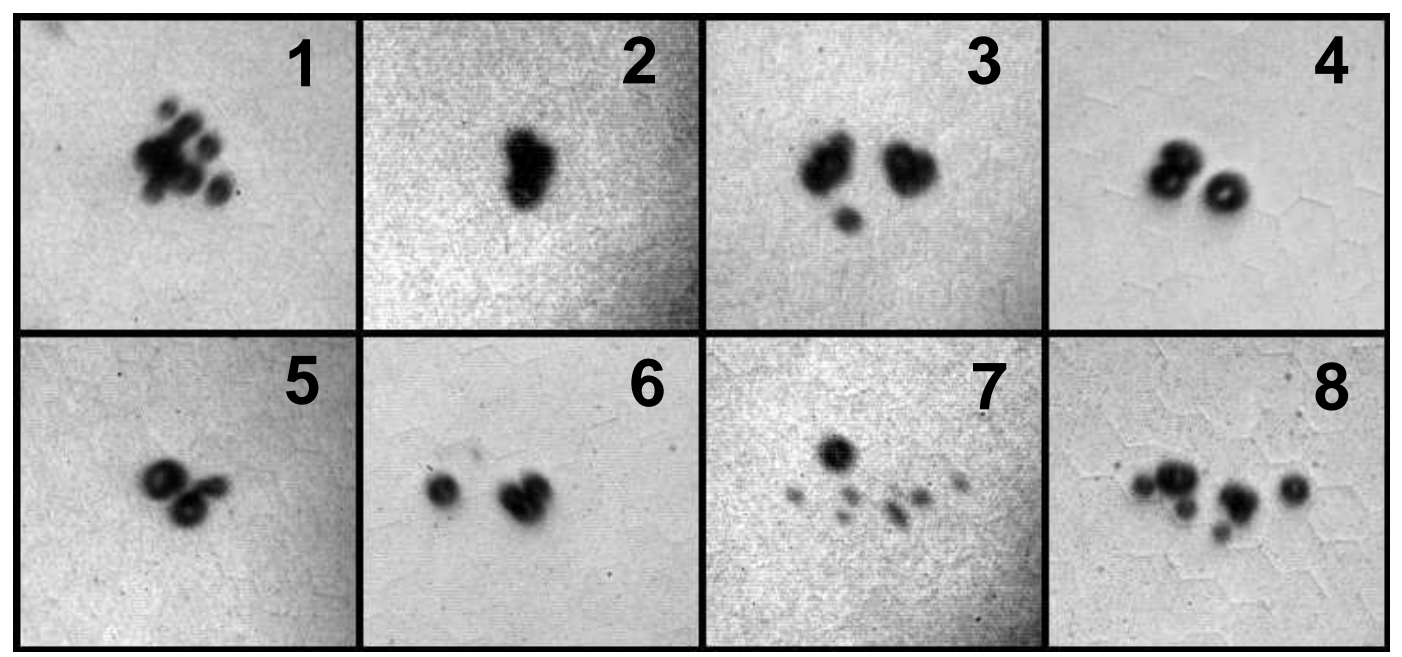

Abbildung 6.2: Clusterstruktur in der Phase maximalen Aufschwingens im zeitlichen Abstand von 1 Periode der Anregungsfrequenz.

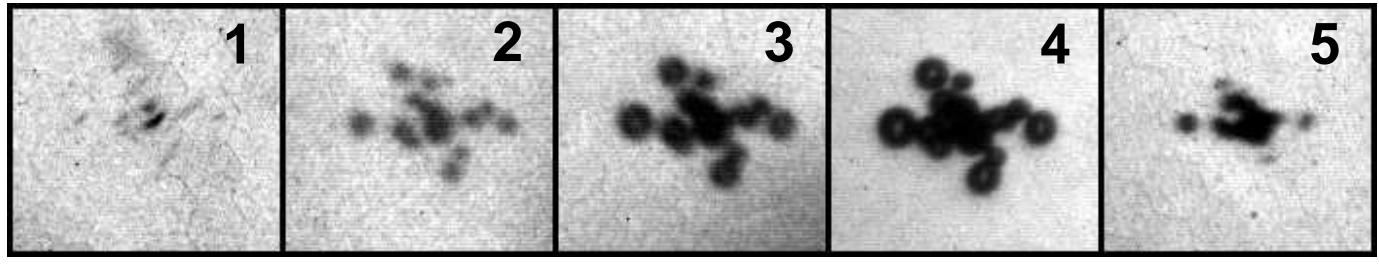

Abbildung 6.3: Aufschwingen und Kollaps einer Clusterstruktur im Abstand von $\mathrm{dt}=\mathrm{T} / 8$. In einem Zeitraum von $3 \mathrm{~T} / 8$ nach dem Kollaps (Bild 6-8) ist die Struktur nicht sichtbar.

Die Ausdehnung und Maximalradien liegen in der Größenordnung der von Luther im Rahmen der akustischen Lichtenbergfigur beschriebenen Cluster [71], die eine etwas größere Ausdehnung $R_{c} \leq 0.5 \mathrm{~mm}$ und höhere Fragmentanzahl von bis zu 30 Einzelblasen haben. Der Cluster wird bei einer Anregungsamplitude $\left(U_{F G}=3.2 \mathrm{Vpp}\right)$, die einer Schalldruckamplitude des blasenfreien Resonators von $p_{a}=220 \mathrm{kPa}$ entspricht, zum Küvettenboden gezogen und zerstört. Es kann bei der gleichen Anregungsamplitude ein neuer Cluster injiziert werden, der erst beim Anschwingen von Schwerewellen zerstört wird $\left(U_{F G}=4.9 \mathrm{Vpp}\right)$. Diese setzen in Abwesenheit des Clusters bereits bei $p_{a}=250 \mathrm{kPa}$ ein $\left(U_{F G}=4.0 \mathrm{Vpp}\right)$, 


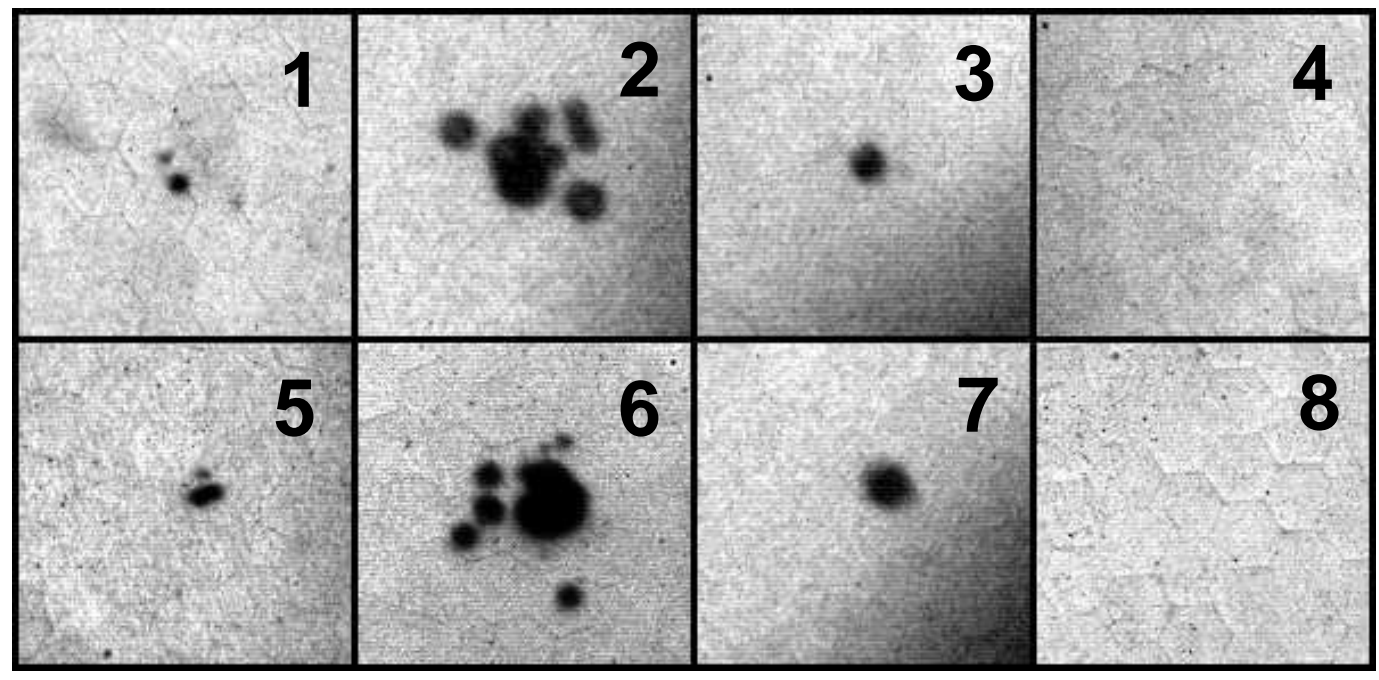

Abbildung 6.4: Pulsationsdynamik der Cluster-Struktur über zwei Perioden der Anregungsfrequenz.

weshalb für diesen Bereich keine Schalldruckamplitude angegeben werden kann. Nimmt man ein lineares Verhältnis zwischen Anregungs- und Schalldruckamplitude an, so wird der Cluster erst bei einer mittleren Schalldruckamplitude von $p_{a}=310 \mathrm{kPa}$ zerstört. Allerdings wird die Dynamik im Resonator bei Einsetzen der Schwerewellen maßgeblich durch diese bestimmt, da der Schalldruck um bis zu 50 \% variieren kann. Dieser bei höherer Schalldruckamplitude injizierte Cluster nimmt seine Position nicht mehr im Schalldruckzentrum ein sondern ist asymmetrisch auf der Diagonalen verschoben. Injiziert man im oberen Stabilitätsbereich des letztbeschriebenen Clusters $\left(U_{F G}=5.0 \mathrm{Vpp}\right)$ mehrere Wassertropfen auf die Oberfläche, so entsteht ein Clusterpaar. In einer Raumrichtung befinden sich die Cluster dort, wo eine $n=2$ Mode in dieser Ausbreitungsrichtung ihre Schalldruckbäuche hätte. In der anderen Raumrichtung befinden sie sich asymmetrisch angeordnet auf einer Seite der Küvette, was möglicherweise auf ein Zusammenspiel mehrerer Resonatormoden zurückzuführen ist. In Abbildung 6.5 ist ihre ungefähre Orientierung im Schallfeld wiedergegeben. 


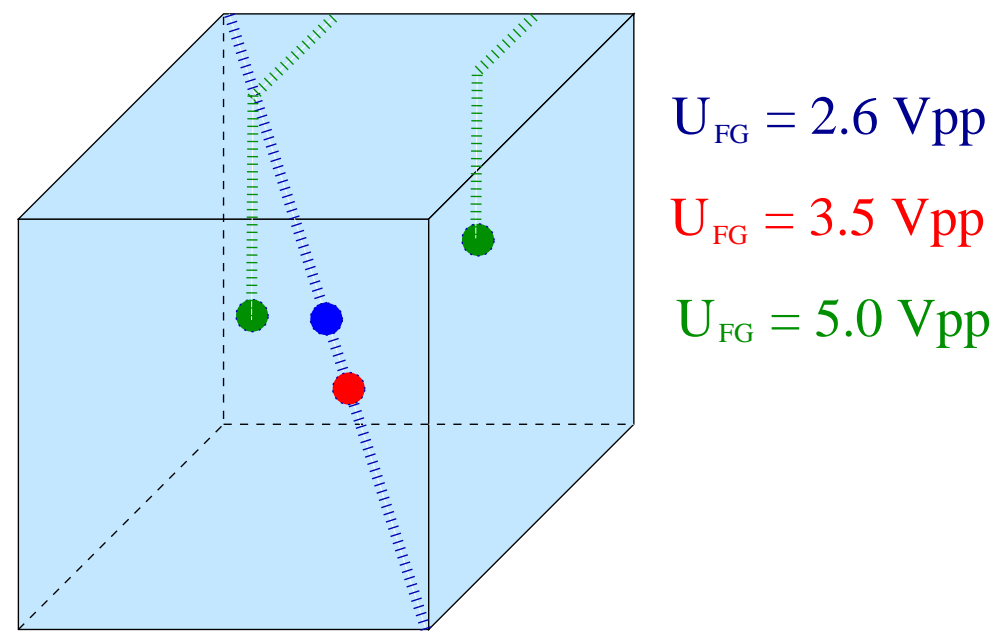

Abbildung 6.5: Schematische Darstellung der räumlichen Orientierung der Cluster bei verschiedenen Anregungsamplituden.

\subsubsection{Akustisches Spektrum}

Abbildung 6.6 fasst die gemittelten normierten Amplitudenspektren des Schallsignals zusammen. Zusätzlich zu den Messungen an reinen Clusterstrukturen (a-c) ist das Spektrum bei Anwesenheit eines Streamers, der sich von einer spontan gebildeten Blasenquelle in der Mitte einer Seitenwand der Küvette bis zum Cluster erstreckt, wiedergegeben (d).

Neben den höheren Harmonischen treten weitere Linien im Spektrum auf. Insbesondere im tieffrequenten, vergleichsweise rauscharmen Teil des Spektrums erscheinen eine Reihe von Linien. Diese liegen zwar oft in der Nähe von Frequenzen, die in einfachem rationalen Verhältnis zur Anregungsfrequenz liegen, eine höhere Aufösung zeigt jedoch, dass diese gemessenen Linien keine Subharmonischen sind. In Abbildung 6.7 ist das Spektrum unterhalb der Anregungsfrequenz vergrößert dargestellt. Ein Vergleich mit der Resonanzkurve des blasenfreien Resonators zeigt, dass alle Linien dort wiederzufinden sind. Dabei handelt es sich vermutlich um durch Wandschwingungen verursachte Resonanzen (siehe Abschnitt A.1). Erstaunlich ist, dass der Rauschpegel in diesem Frequenzbereich vergleichsweise gering ist. Ein signifikanter Rauschpegel hätte die Anfachung der Resonanzen erklären können. Konsistent mit der Vermutung, dass es sich um Wandschwingungen handelt, ist die Tatsache, dass die Amplituden der niederfrequenten Moden in Anwesenheit einer Blasenquelle an der Wand (d) bei gleicher Anregung um bis zu 2 Größenordnungen anwachsen. 
Auch das höherfrequente Spektrum verändert sich mit steigender Anregungsamplitude. So ist beim sich zentriert positionierenden Cluster $\left(U_{F G}=2.6 \mathrm{Vpp}\right)$ die dritte Harmonische stärker angeregt als die zweite, was sich bei höherer Anregungsamplitude $\left(U_{F G}=3.5 \mathrm{Vpp}\right)$ umkehrt. Der Anteil der zweiten Harmonischen dominiert beim Clusterpaar sogar über die Anregungsamplitude $\left(U_{F G}=5.5 \mathrm{Vpp}\right)$, die am Ort des Hydrofons bei niedrigerer Anregung eine Größenordnung über der zweiten Harmonischen liegt. In Anwesenheit des Streamers dominiert nicht mehr die zweite Harmonische, sondern eine etwas höhere Frequenz. Der Abstand zur zweiten Harmonischen beträgt $3.2 \mathrm{kHz}$, was gerade die Frequenz der niedrigsten im Spektrum auftauchenden Linie (nahe $f_{\text {res }} / 8$ ) ist, die in Anwesenheit des Streamers stark anschwingt. Die gleich hohe Linie auf der anderen Seite der dominierenden Frequenz im gleichen Frequenzabstand lässt auf eine Amplitudenmodulation schließen. Weder der stationäre Cluster noch die stationäre Streamer-Cluster-Konfiguration zeigen also subharmonische Anteile. Auch halbzahlige höhere Harmonische $(3 / 2,5 / 2,7 / 2,9 / 2)$ können nicht eindeutig identifiziert werden.

Das Amplitudenverhältnis der höheren Harmonischen kann in Zusammenhang mit der Clusterposition gebracht werden: Je stärker die zweite Harmonische anschwingt, desto stärker wird der Cluster aus dem Küvettenzentrum gezogen. Hier scheint die Wechselwirkung zwischen nichtlinearer Blasenschwingung und der Anregung höherer Resonatormoden, eventuell gepaart mit einem repulsiven Schalldruckbauch der Grundmode, für die Positionsveränderung verantwortlich zu sein. 


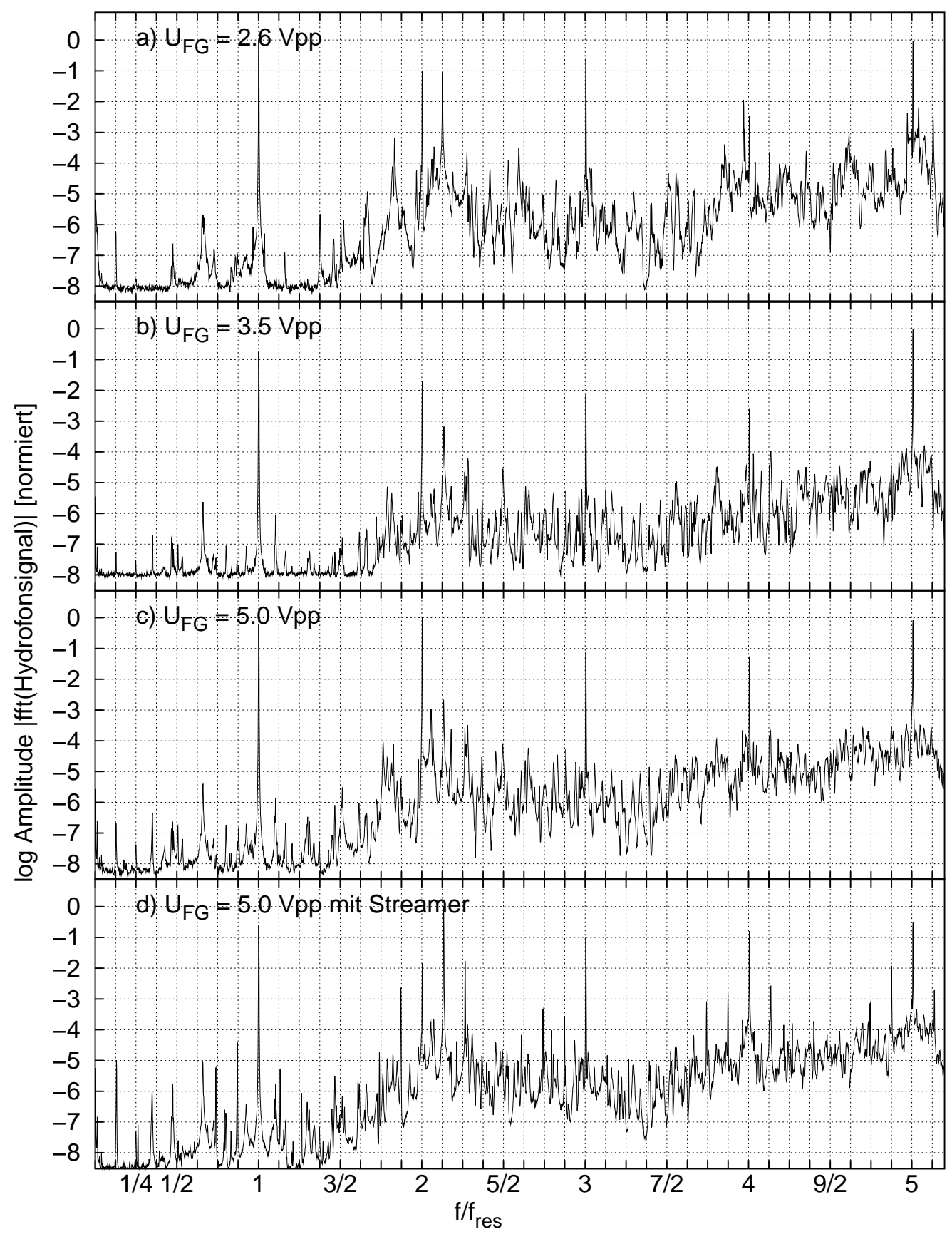

Abbildung 6.6: Amplitudenspektrum des akustischen Signals stationärer Cluster bei verschiedenen Anregungsamplituden. 


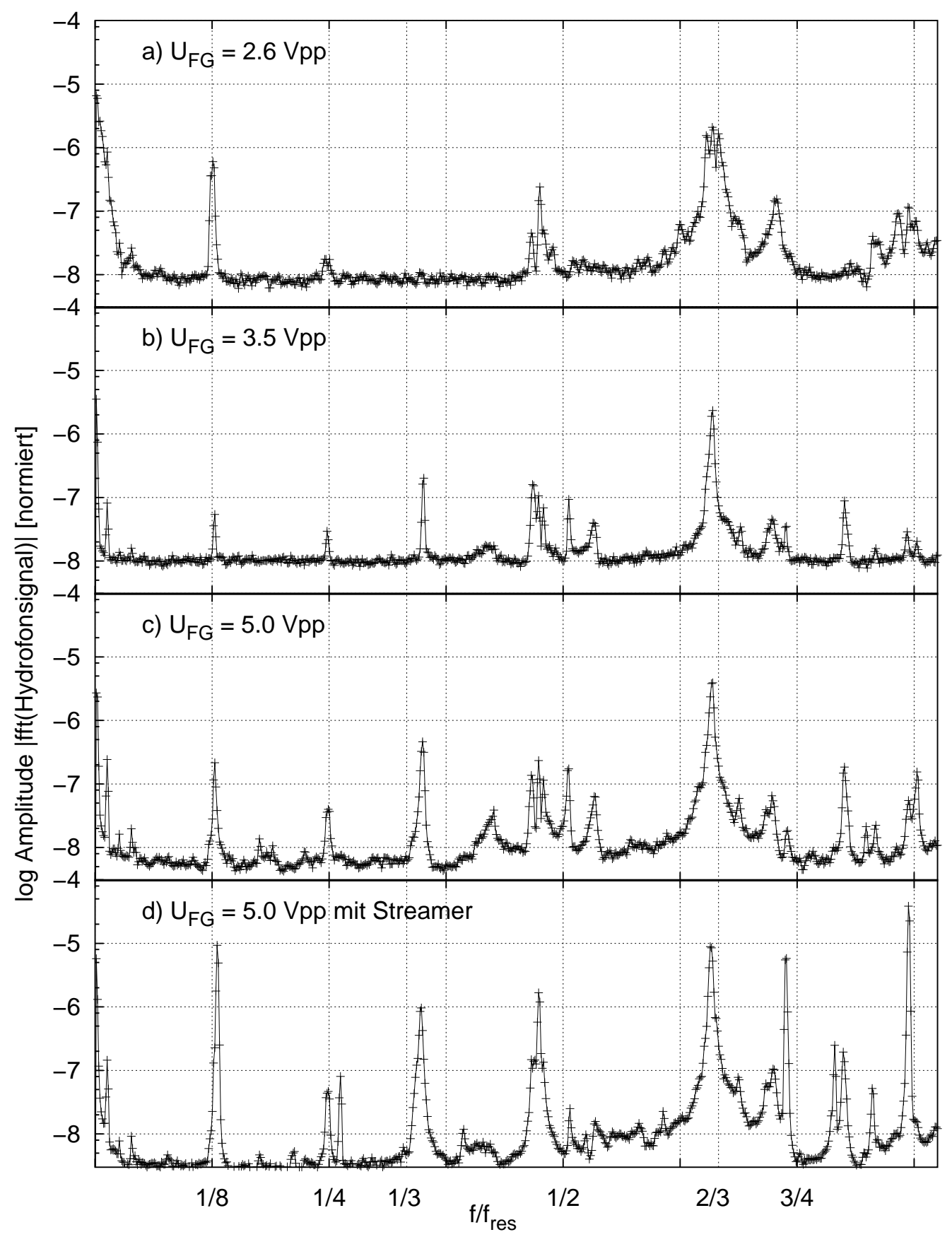

Abbildung 6.7: Tieffrequenter Ausschnitt aus Abbildung 6.6. 


\section{Kapitel 7}

\section{Streamerstrukturen}

Streamer erscheinen bei der Betrachtung als filamentartige Strukturen. In ihnen bewegen sich Blasen in Richtung des Schalldruckbauches, wobei die bevorzugten Pfade über längeren Betrachtungszeiträume (von Sekunden bis Minuten) ortsfest sind. Sie wurden bereits 1949 von Blake beschrieben, der auch den Begriff „streamer "für die filamentartigen Blasentrajektorien eingeführt hat [10]. Die bekannteste Streamerstruktur in Kavitationsfeldern ist die akustische Lichtenbergfigur, in der alle Filamente in einem gemeinsamen Zentrum enden, das sich im Schalldruckbauch des Stehwellenwellenfeldes befindet [83]. Umfassende Untersuchungen zur akustischen Lichtenbergfigur sind von Luther [71] durchgeführt worden. In Bezug auf die Blasenquellen zeigten kürzlich erstellte stereokinematografische Hochgeschwindigkeitsaufnahmen von akustischen Lichtenbergfiguren, dass ein Großteil der Blasen aus der Richtung einer Klebekante des verwendeten Plexiglasquaders kamen [3] (Zur Beschreibung des Resonators siehe A.2). In diesem Resonator ist auch meist eine starke Unsymmetrie der Struktur in Richtung des Küvettenbodens zu beobachten, an dem der Schallwandler befestigt ist. Aufnahmen aus diesem Resonator finden sich in dieser Arbeit in Abschnitt 8.2, Abbildung 8.13 und Kapitel 9, Abbildung 9.4 und 9.6. Die Tatsache, dass im Glaswürfel (siehe A.1) bei gleicher Schalldruckamplitude keine vergleichbar große und stabile Lichtenbergfigur erzeugt werden kann, deutet auf die Wichtigkeit der Blasenquellen für die Ausbildung der Streamerstrukturen hin. Die Glasküvette zeichnet sich durch Abwesenheit von Störstellen an den Wänden aus, da diese industriell geschweisst sind. In diesem Resonator können Blasenquellen allerdings künstlich erzeugt werden. Insbesondere kann damit eine räumliche Dimensionsreduktion vorgenommen werden, was die gezielte Untersuchung einzelner Streamer ermöglicht. 


\subsection{Eindimensionaler Streamer}

Die einfachst mögliche Streamerstruktur besteht aus einem Filament, also einer einzigen Blasenspur und kann durch die Erzeugung einer Blasenquelle erzeugt werden. Hierzu wird eine Nadel im Glasquader senkrecht über dem Zentrum positioniert, es bildet sich ein senkrechtes Filament von der Nadel zum Zentrum. Die horizontalen Abweichungen betragen weniger als $1 \mathrm{~mm}$. Abbildung 7.1 zeigt eine Langzeitaufnahme eines eindimensionalen Streamers. Die Blasen laufen von der Nadelspitze (Pfeil) ins Zentrum der Küvette. Da die Blasen in der oberen Hälfte des Filaments nicht stark aufschwingen und eine vergleichsweise hohe Geschwindigkeit besitzen, sind dort kaum Blasen zu sehen. Die im Umfeld des Streamers sichtbaren weißen Punkte sind im Wasser befindliche Partikel.

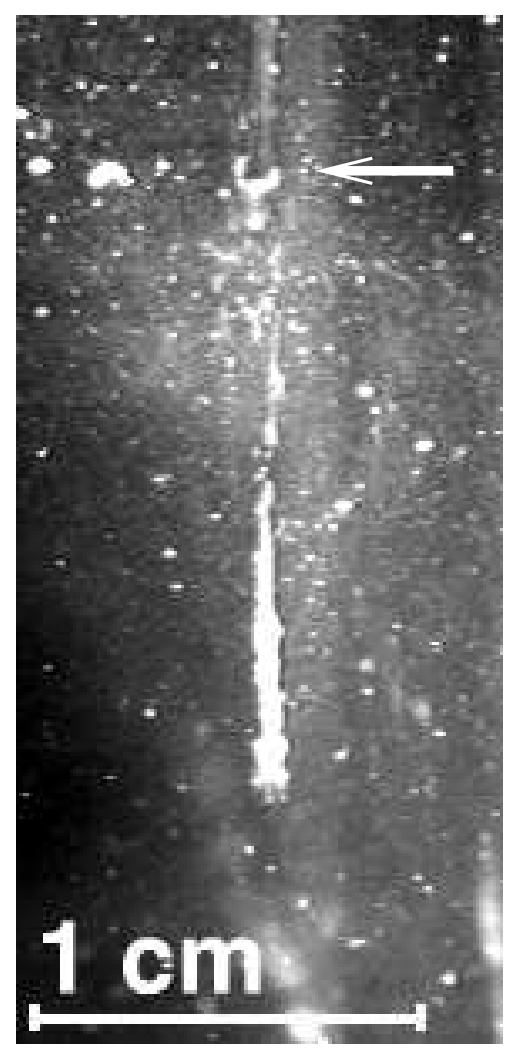

Abbildung 7.1: Langzeitbelichtung eines eindimensionalen Streamers. Es bildet sich ein Blasenschleier an der Nadelspitze (Pfeil). 


\subsubsection{Durchführung}

Zur Untersuchung der Translationsdynamik wird der Streamer mit einer Bildwiederholrate von $500 \mathrm{~Hz}$ aufgenommen. Simultan wird das Vibrometersignal von dem 16-bit A/D-Wandler aufgezeichnet. Die maximale Aufnahmelänge von $2 \mathrm{~s}$ ist durch den Speicher des A/D-Wandlers begrenzt. Die Bilder werden zunächst bandpassgefiltert, anschließend wird die Blasenposition ermittelt (bezüglich des Verfahrens siehe [71]). Danach werden die Trajektorien der Blasen mit Particle Image Velocimetry bestimmt. Aus dem Schallsignal werden Kurzzeitspektren erzeugt, die in ihrer Fensterlänge mit dem Bildabstand übereinstimmen. Die Daten werden vor der Fouriertransformation mit einem flattop-Fenster multipliziert.

\subsubsection{Blasenpositionen}

Abbildung 7.2 zeigt die vertikale Aufenthaltswahrscheinlichkeit der Blasen für verschiedene Anregungsamplituden $U_{F G}$. Der Anschaulichkeit halber ist hier der Koordinatenursprung an den Ort der Nadelspitze gesetzt, die positive z-Achse zeigt nach unten in Richtung des Schalldruckbauches, der sich bei z $=15 \mathrm{~mm}$ befindet.

Bei vergleichsweise niedrigem Schalldruck $\left(p_{a}=116 \mathrm{kPa}\right)$ befindet sich ein Großteil der Blasen im Schalldruckbauch. Die ersten Blasen können etwa 8 mm oberhalb des Zentrums detektiert werden. Dort wird offensichtlich die dynamische Blakeschwelle für große Pulsationsamplituden überschritten, die theoretisch mit der Grenze für hohe Diffusionsraten zusammenfällt [70]. Bei Erhöhung der Anregungsamplitude wird die primäre Bjerkneskraft für eine zunehmende Menge von auftretenden Blasenradien am Druckbauch repulsiv [2]. Der Schalldruckbauch wird entvölkert, gleichzeitig sind die Blasen früher detektierbar. Bei einer Anregungsamplitude von $U_{F G}=7 \mathrm{Vpp}\left(p_{a}=180 \mathrm{kPa}\right.$, Bild (a)) ist das Zentrum völlig blasenfrei. Bei weiterer Amplitudenerhöhung ( $U_{F G}=8 \mathrm{Vpp}, p_{a}=192 \mathrm{kPa}$, Bild (b)) gelangen jedoch zunehmend wieder Blasen in den Schalldruckbauch und es bildet sich ein zweites Maximum der Aufenthaltswahrscheinlichkeit. Die Blasen erscheinen in der Streulichtaufnahme als sehr helle und ausgedehnte Objekte und Aufnahmen mit dem Fernmikroskop zeigen, dass es sich nicht um vereinzelte Objekte, sondern um Blasencluster handelt (siehe Kapitel 6). Diese spüren als komplexe Struktur anscheinend eine andere primäre Bjerkneskraft. Bei höheren Anregungsamplituden wird auch für die Cluster der Schalldruckbauch repulsiv und sie nehmen - analog zu den Einzelblasen - eine Position oberhalb des Zentrums ein. 


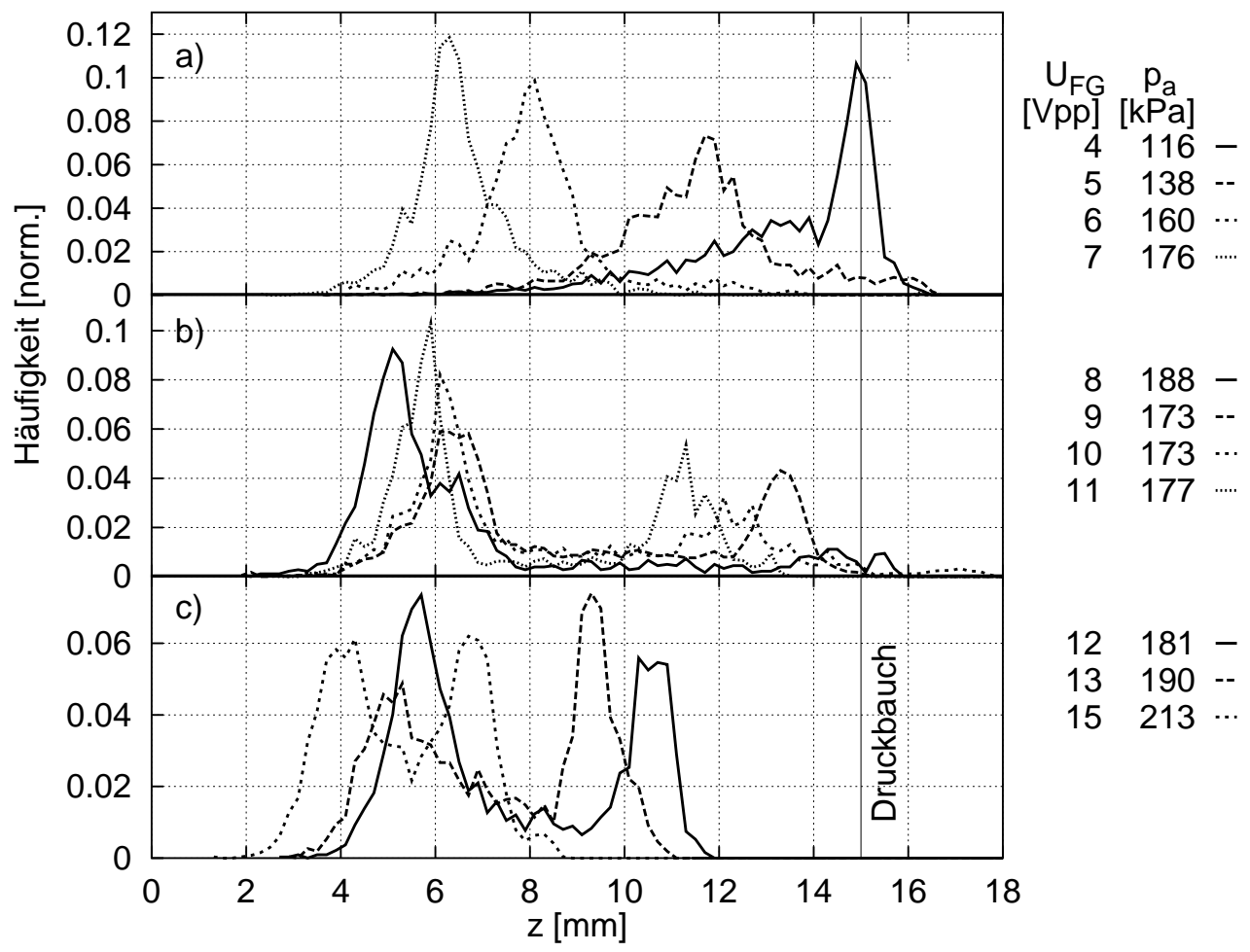

Abbildung 7.2: Aufenthaltswahrscheinlichkeit einer Blase im eindimensionalen Streamers bei ansteigenden Anregungsamplituden $U_{F G}$. Zusätzlich sind die ermittelten Schalldruckamplituden $p_{a}$ angegeben.

\subsubsection{Blasengeschwindigkeiten}

Die im Filament auftretenden Geschwindigkeiten sind in Abbildung 7.3 zusammengefasst. Dargestellt ist die normierte Häufigkeitsverteilung der Vertikalgeschwindigkeiten. Zusätzlich sind die mittlere Geschwindigkeit $\overline{v_{z}}$ (vertikale durchgezogene Linie) und die mittlere Absolutgeschwindigkeit $\overline{\left|v_{z}\right|}$ (gestrichelte Linie) eingezeichnet. Die Mittelung erstreckt sich dabei über alle im Graphen dargestellten Messungen. Ein Vorteil des eindimensionalen Streamers ist die einfache Unterscheidung der Bewegungsrichtung. Im dreidimensionalen Streamer kann dies theoretisch auch erreicht werden, erfordert allerdings eine Behandlung in sphärischen Koordinaten und eine exakte Kenntnis der Zentrumsposition. Insgesamt sieht man bei allen Anregungsamplituden eine bevorzugte Bewegung zur Küvettenmitte. Der beträchtliche negative Geschwindigkeitsanteil insbesondere bei Abwesenheit der Cluster wird durch rücklaufende Blasen verursacht. Solange der Schalldruckbauch attraktiv ist, wird dies in erster Linie durch die sekundäre Bjerkneskraft verursacht werden. Bei höheren Anregungsamplituden können auch 


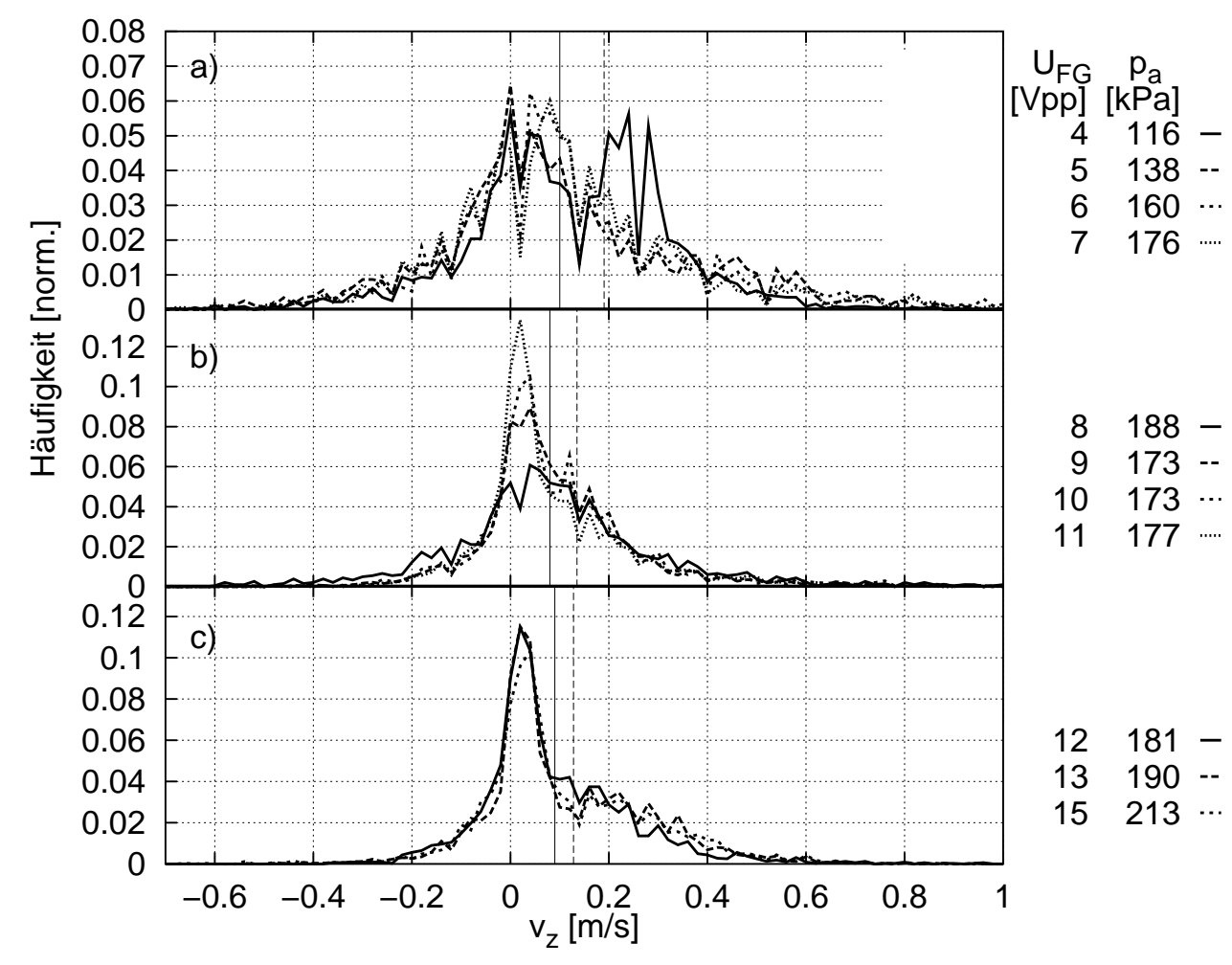

Abbildung 7.3: Histogramm der Vertikalgeschwindigkeiten eines eindimensionalen Streamers für ansteigende Anregungsamplituden (siehe Abbildung 7.2). Die Häufigkeiten wurden auf die Gesamtzahl der ermittelten Geschwindigkeiten der jeweiligen Messung normiert. Die durchzogene vertikale Linie bezeichnet die mittlere Geschwindigkeit $\overline{v_{z}}$ der dargestellten Messungen, die gestrichelte Linie die mittlere Absolutgeschwindigkeit $\overline{\left|v_{z}\right|}$.

Blasen, die durch Kollision oder Diffusion stark anwachsen, durch die repulsive primäre Bjerkneskraft in Gegenrichtung beschleunigt werden. Unter der Annahme, dass der durch die sekundäre Bjerkneskraft verursachte Geschwindigkeitsanteil in beiden Richtungen gleich ist, gibt der Mittelwert der Geschwindigkeit $\overline{v_{z}}$ die Nettogeschwindigkeit in Richtung des Schalldruckbauches an. Dieser zeigt keine Tendenz bezüglich der Anregungsamplitude und liegt gemittelt über alle Messreihen bei $\overline{v_{z}}=(0.09 \pm 0.01) \mathrm{m} / \mathrm{s}$. Der Mittelwert der Absolutwerte der Geschwindigkeiten $\overline{\left|v_{z}\right|}$ hingegen zeigt insgesamt eine abnehmende Tendenz. 


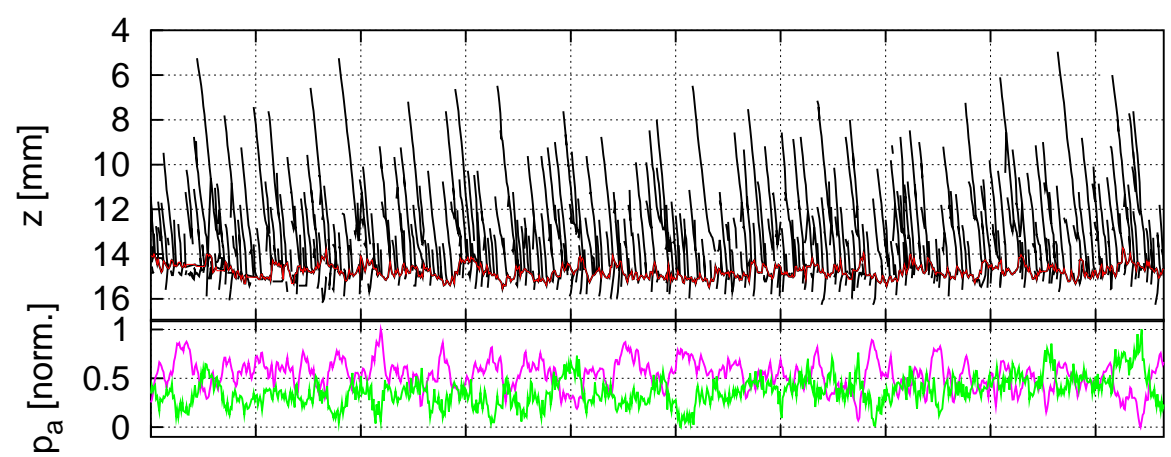

a) $116 \mathrm{kPa}$

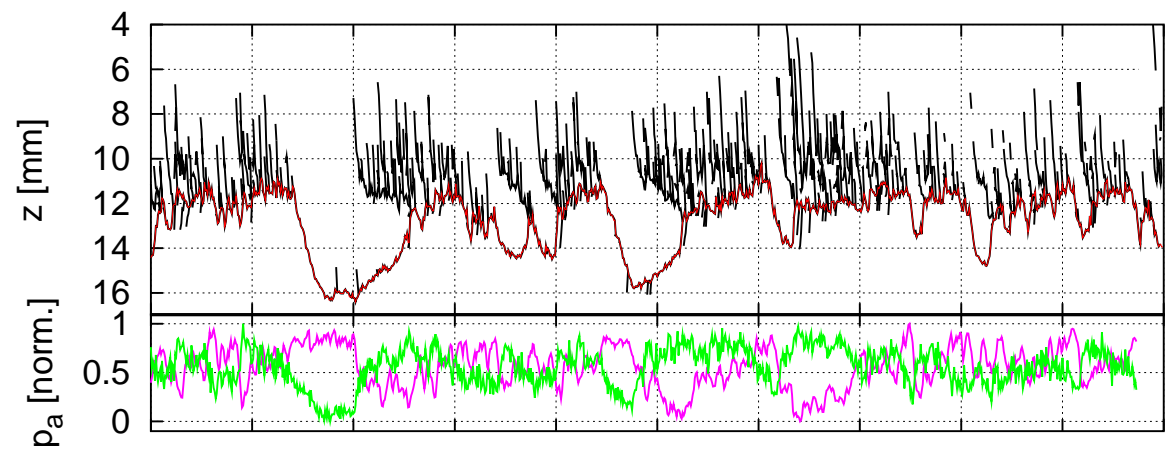

b) $138 \mathrm{kPa}$

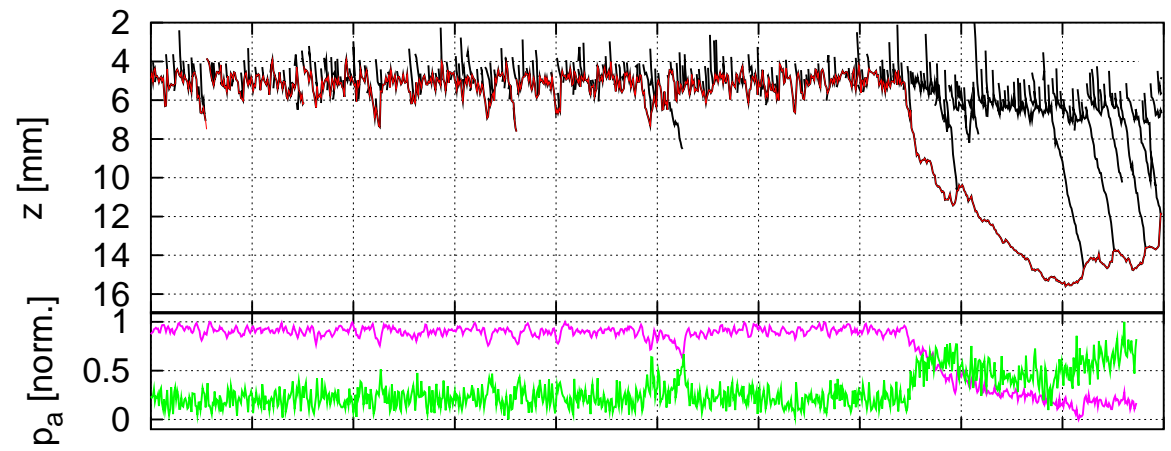

c) $188 \mathrm{kPa}$

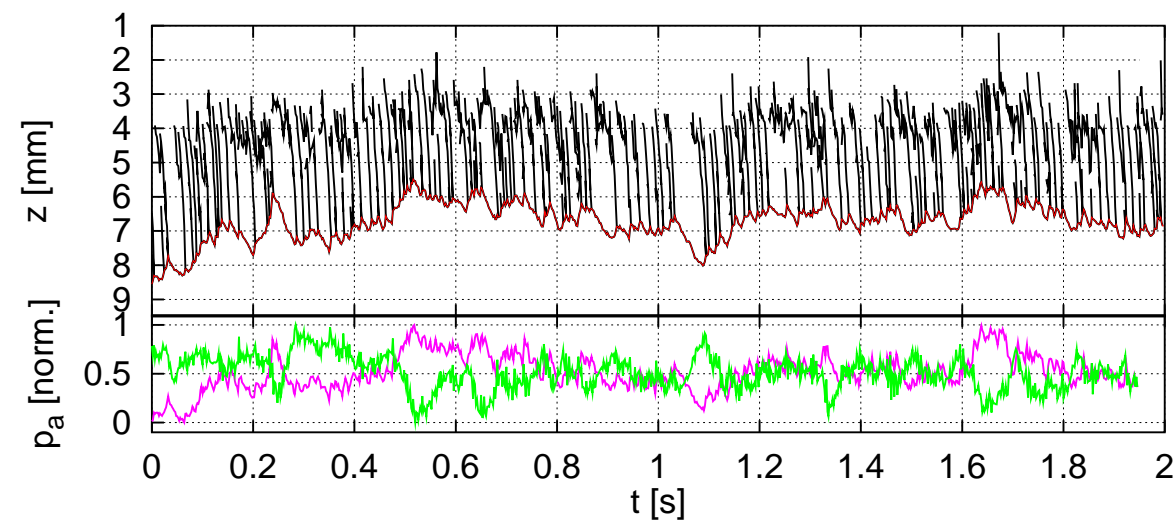

d) $213 \mathrm{kPa}$

Abbildung 7.4: Zeitlicher Verlauf von Blasenpositionen (schwarze Linien) und der Amplitude der Anregungsfrequenz (magenta) sowie der zweiten Harmonischen (grün). Die Trajektorie der zentralen Blase bzw. des zentralen Blasenclusters ist rot eingezeichnet. 


\subsubsection{Akustisches Spektrum}

Im Vibrometersignal wird bei keiner Messung das Anschwingen der Subharmonischen beobachtet. Es zeigen sich bei konstanter Anregungsamplitude temporale Amplitudenschwankungen sowohl in der Grundfrequenz als auch in den höheren Harmonischen.

In Abbildung 7.4 sind für ausgewählte Messungen sowohl der zeitliche Verlauf sämtlicher detektierter Blasenpositionen (schwarze Linien) als auch die normierte Schalldruckamplitude der Grundfrequenz $f_{0}$ (rot) und der zweiten Harmonischen (grün) dargestellt. Generell zeigt sich ein gegenläufiges Amplitudenverhältnis zwischen Grundfrequenz und zweiter Harmonischer. Der Verlauf der nächst höheren Harmonischen (dritte bis fünfte Harmonische) ist ähnlich wie der der zweiten Harmonischen. Die Amplitudenabnahme der Anregungsfrequenz wird also nicht nur durch die Veränderung der Grundresonanz des Gesamtsystems (siehe Kapitel 5.1) verursacht, sondern mit einem Teil der Schallenergie werden höhere Harmonische erzeugt. Bei hohen Anregungsamplituden (Bild (c) und (d)) besteht ein direkter Zusammenhang zwischen der Position des zentralen Blasenclusters (rot markiert) und der Schalldruckamplitude. Je näher der Cluster dem Zentrum kommt, desto stärker wird die zweite Harmonische angeregt, die Amplitude der Grundfrequenz fällt. In Bild (b) ist ebenfalls gelegentlich die Bewegung einer Blase ins Zentrum zu beobachten. Zeitgleich stoppt der Blasenfluss von der Nadel (siehe zum Beispiel t $=0.3-0.5 \mathrm{~s}$ ). Hier ist die Bewegung zum Zentrum vielmehr mit einer Abnahme der zweiten Harmonischen verbunden. Erst wenn neue Blasen sichtbar werden, steigt die Amplitude der zweiten Harmonischen erneut an. Bei vergleichsweise niedrigen Schalldruckamplituden (Bild (a)) ist ein Zusammenhang zwischen Schalldruckverlauf und Blasenpositionen nicht erkennbar. Dort ist die Variabilität der Position der zentralen Blase deutlich geringer. Zudem entstehen in dieser Messung - vermutlich durch Aufschwingen abgespaltener Fragmente - auch knapp unterhalb des Schalldruckbauches Blasen, wodurch eine zentrale Blase nicht eindeutig bestimmbar ist.

In Abbildung 7.5 ist für die gesamte Messreihe die Schalldruckamplitude der Anregungsfrequenz gegen die Position der zentralen Blase aufgetragen. Für Messungen, bei denen einzelne Blasen dominieren (schwarze Punkte) zeigt sich eine sichtbare, aber schwache Abhängigkeit der Schalldruckamplitude von der Blasenposition innerhalb einer Messung. Der zentrale Cluster hingegen (farbige Punkte) zeigt einen eindeutigen Zusammenhang zwischen Clusterposition und Schalldruckamplitude, die innerhalb einer Messung um bis zu 10 Prozent schwanken kann. 


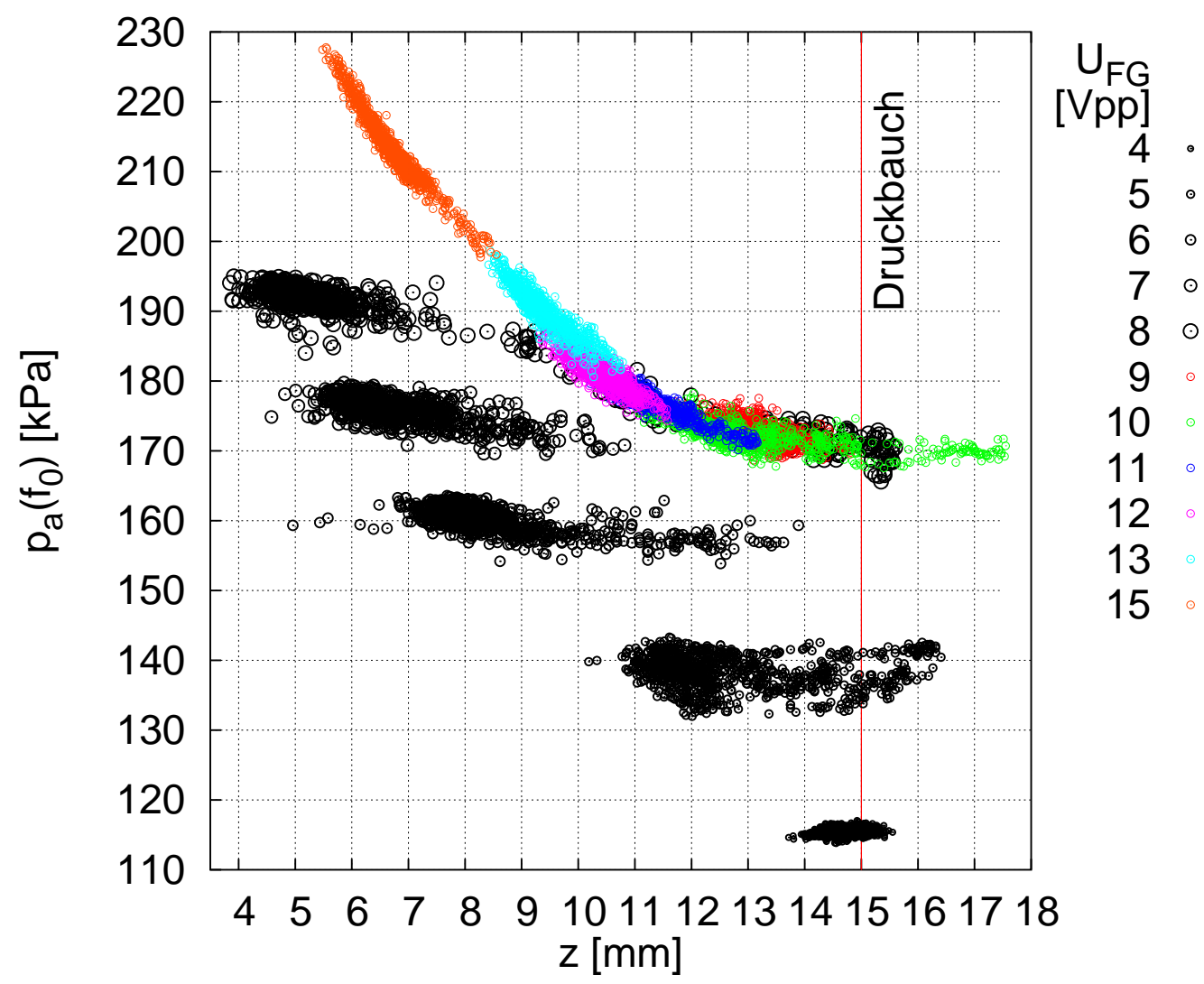

Abbildung 7.5: Schalldruckamplitude der Anregungsfrequenz in Abhängigkeit der vertikalen Position der zentralen Blase bzw. des zentralen Blasenclusters.

Das Vorzeichen der primären Bjerkneskraft ist bei gegebenem Radius und bekannter Richtung des Schalldruckgradienten nur vom Schalldruck abhängig (siehe Abschnitt 2.2). Insbesondere kann für jeden Blasenradius $R_{0}$ die Grenzamplitude $p_{a, b j 1}$, bei der die primäre Bjerkneskraft verschwindet, numerisch bestimmt werden. In Abbildung 7.6 sind die Linien gleicher Schalldruckamplitude für ein ideales Stehwellenfeld zusammen mit den gemessenen Positionen und Schalldruckamplituden des zentralen Clusters eingezeichnet. Der Cluster befindet sich im Wesentlichen an Orten, an denen die Schalldruckamplitude in etwa $p_{a}(z)=170 \mathrm{kPa}$ beträgt. Dies entspricht der Grenze von verschwindender Bjerkneskraft für Blasen mit einem Ruheradius von etwa $10 \mu \mathrm{m}$ (Simulation von R. Mettin). Dies deutet darauf hin, dass der Cluster eine Art effektiven Ruheradius hat, der über den gemessenen Schalldruckbereich weitgehend konstant ist. Mit diesem effektiven $\mathrm{Ru}-$ heradius kann zumindest die Blasenposition im Rahmen der Einzelblasentheorie 
konsistent beschrieben werden.

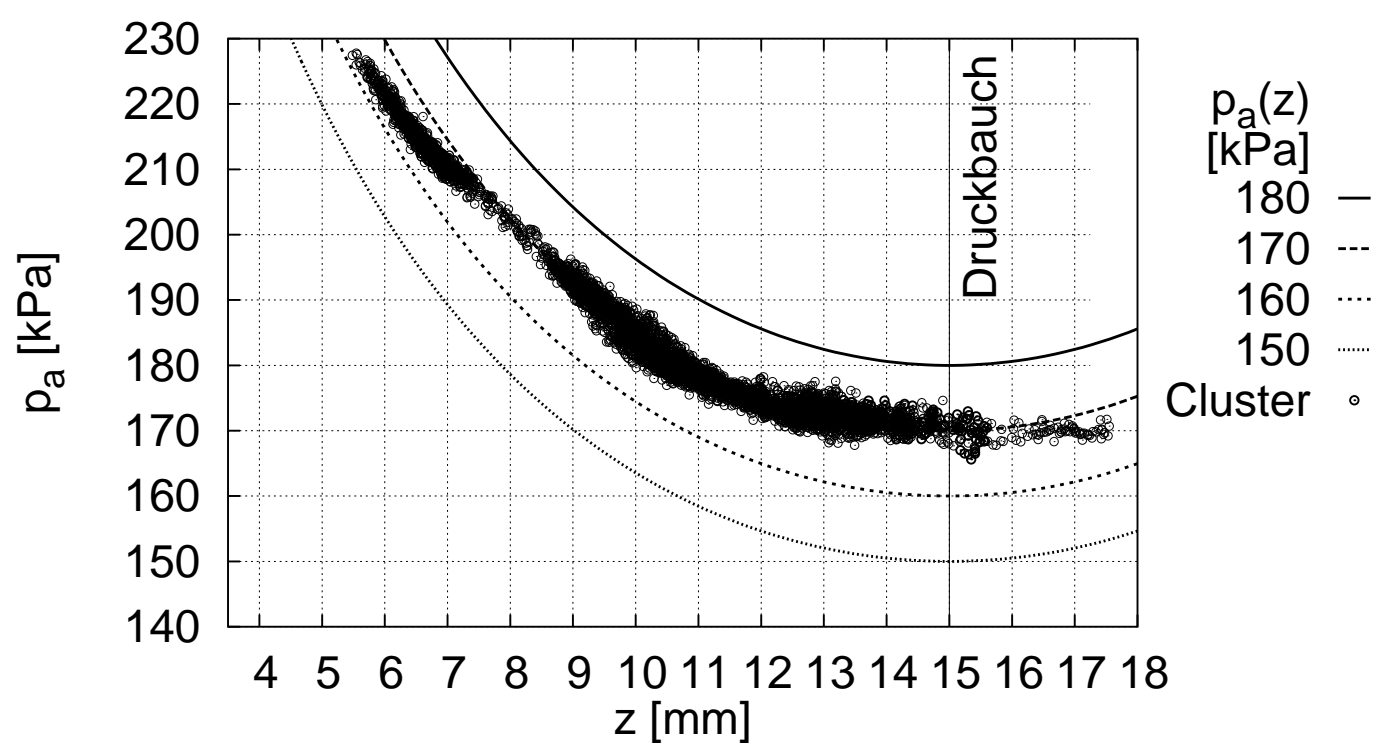

Abbildung 7.6: Ausgewählte Schalldruckamplituden $p_{a}(z)$ in Abhängigkeit von der vertikalen Position $z$ im Resonator und der Schalldruckamplitude $p_{a}$ im Zentrum der Küvette. Die Punkte bezeichnen die gemessenen Orte und Schalldruckamplituden für den zentralen Cluster. 


\subsection{Zweidimensionale Streamerstrukturen}

Durch gezieltes Induzieren von Blasenquellen können verschiedene Streamerstrukturen erzeugt werden. Im Folgenden werden einige Strukturen vorgestellt, die für weitere Untersuchungen der Dynamik von Kavitationsstrukturen interessant sind.

\subsubsection{Dreiarmiger Streamer}

An den Resonatorwänden können Blasen induziert werden. Generell liefern die Blasenquellen an der Wand nur wenige Blasen. So entstehen eher fragile Strukturen, bei denen die Streamer oft bis zu Sekunden unterbrochen sind. In Abbildung 7.7 sind einmal alle Streamer aktiv (mittleres Bild) und jeweils ein Seitenstreamer unterbrochen (links und rechts). Zwischen den Bildern liegen 0.5 - 1 s.

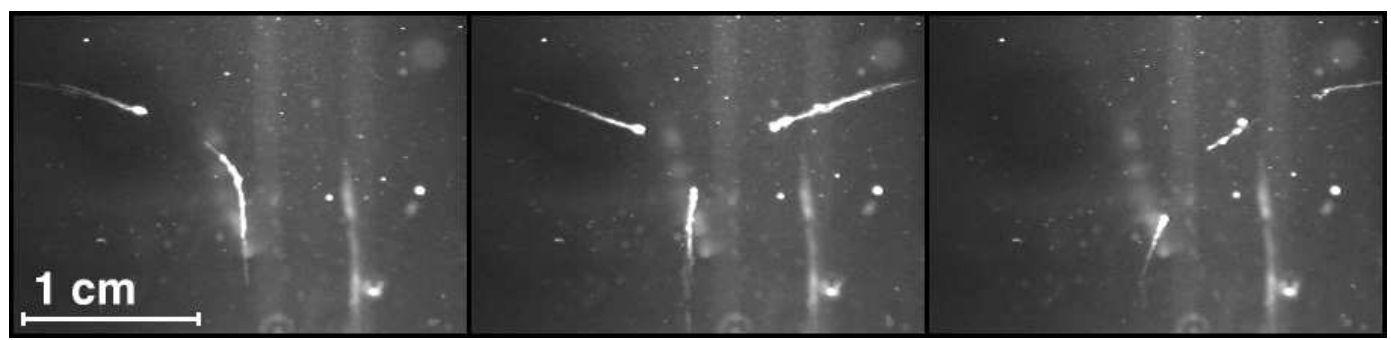

Abbildung 7.7: Zweidimensionale Streamerkonfiguration, die Blasenquellen befinden sich an den Seitenwänden und am Resonatorboden, Bildabstand 0.5 - $1 \mathrm{~s}$, Belichtungszeit $16 \mathrm{~ms}$.

\subsubsection{Vierarmiger Streamer}

Aufgrund der fehlenden Wand kann keine resonatoreigene Blasenquelle an der Wasseroberfläche induziert werden. Durch Einbringen einer Nadel kann die Blasenquelle analog zum eindimensionalen Streamer künstlich ergänzt werden. Dies hat zusätzlich den Effekt, dass durch die erhöhte Anzahl an Blasen auch die anderen Filamente in begrenztem Rahmen stabilisiert werden können. Die Streamer sind weiter unterbrochen, aber deutlich blasenreicher, wenn sie aktiv sind. Vermutlich wird dies mittelbar durch die Erhöhung der Mikroblasenanzahl im Wasser verursacht. In dieser Struktur können zum Beispiel Effekte wie die Verschiebung des Strukturschwerpunktes durch die sekundäre Bjerkneskraft isoliert beobachtet werden, wie in Abbildung 7.8 dargestellt ist. 


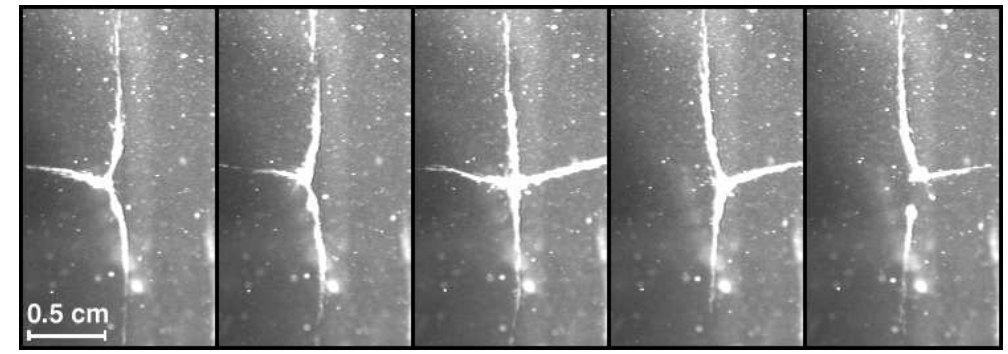

Abbildung 7.8: Vierarmige Streamerkonfiguration, zusätzlich ist eine Nadel etwa $0.7 \mathrm{~cm}$ in den Resonator eingetaucht. Bildabstand 0.1s, Belichtungszeit $16 \mathrm{~ms}$.

\subsubsection{Zweidimensionale akustische Lichtenbergfigur}

Um die Anzahl und möglichen Orte der Blasenquellen zu erhöhen, wird ein Ring aus zwei verdrillten Drähten senkrecht die Mitte des Glaswürfels gehängt. Die Schalldruckamplitude beträgt $p_{a}=120-180 \mathrm{kPa}$. So lässt sich eine zweidimensionale akustische Lichtenbergfigur erzeugen, deren Blasenquellen in etwa äquidistantem Abstand zum Zentrum liegen. Die Streamerfigur zeigt die gleichen Eigenschaften wie die dreidimensionale Streamerstruktur. Die Filamente sind über mehrere Sekunden stabil, können sich aber auch verschieben, versiegen oder es können neue hinzukommen. In Abbildung 7.9 ist die Verschiebung und Verstärkung eines Filamentes durch eine vom Drahtring ausgestoßene Blasenwolke, ab dem zweiten Bild erkennbar als sich vergrößernder Grauschleier, dargestellt. Der Bildabstand beträgt hier $0.1 \mathrm{~s}$, die Belichtungszeit $16.7 \mathrm{~ms}$. Der scheinbare innere Ring ist die Spiegelung auf der Rückwand, die sich leider nicht ohne größeren Aufwand unterdrücken lässt.

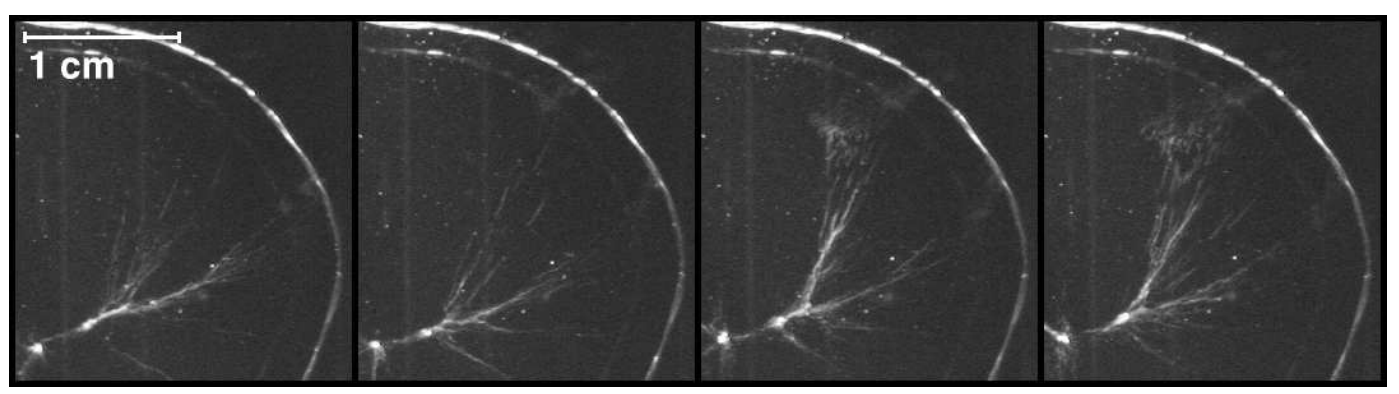

Abbildung 7.9: Verschiebung und Verstärkung eines Filamentes durch eine am Drahtring erzeugte Blasenwolke. Bildabstand $0.1 \mathrm{~s}$, Belichtungszeit $16 \mathrm{~ms}$.

Auch die intermittierende Entvölkerung des Schalldruckbauches kann bei der zweidimensionalen ak 


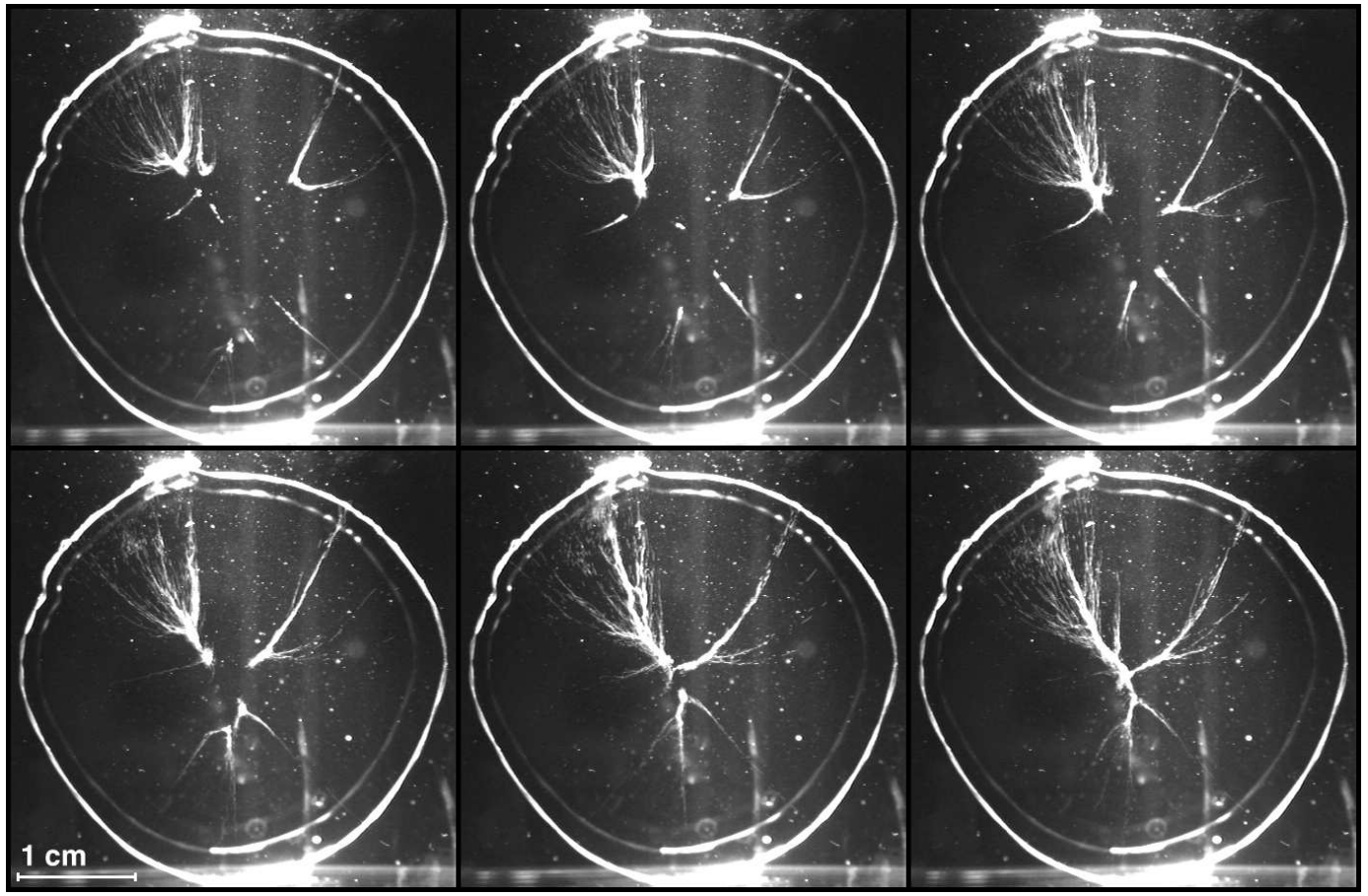

Abbildung 7.10: Intermittierende Entvölkerung des Schalldruckbauches. Bildabstand $0.1 \mathrm{~s}$, Belichtungszeit $16 \mathrm{~ms}$.

Wichtig für die Entstehung einer stabilen zweidimensionalen akustischen Lichtenbergfigur ist die ausreichende, aber nicht übermäßige Anzahl an nichtversiegenden Blasenquellen. Ein einfacher Drahtring liefert nur direkt nach dem Eintauchen einige Blasenquellen, die jedoch nach wenigen Sekunden versiegen. In Abbildung 7.11 ist die Reduzierung der Blasenquellen zu sehen, zwischen den Bildern liegen mehrere Sekunden.

Trotz der Störstellen, die zu Beginn auch als Blasenquellen fungieren, reduziert sich die Streamerstruktur auf ein einzelnes Filament vom Drahtende. Eine Erhöhung der Anregungsamplitude führt zu keinem erhöhten Blasenfluss.

Auch ein Überangebot an Blasen kann die Struktur destabilisieren. Es bildet sich im Zentrum eine sehr große Blase, die das Schallfeld so stark beeinträchtigt, dass die Schalldruckamplitude stark sinkt. Erst wenn diese Blase zerstört ist, werden Blasen wieder beschleunigt. Da die große Blase sehr viele Fragmente zurücklässt, ist dieser Vorgang oft zyklisch. In Abbildung 7.12 ist ein solcher Prozess über mehrere Sekunden verfolgt. Zu Beginn ist eine filamentreiche Streamerstruktur zu sehen. Nach und nach konzentriert sich die Struktur auf das Zentrum, die Blasen in den Filamenten werden langsamer, sie erscheinen nicht mehr als Streaks, sondern einzelne Punkte. Nach 4 Sekunden (Bild 9) ist der Streamer völlig zum 


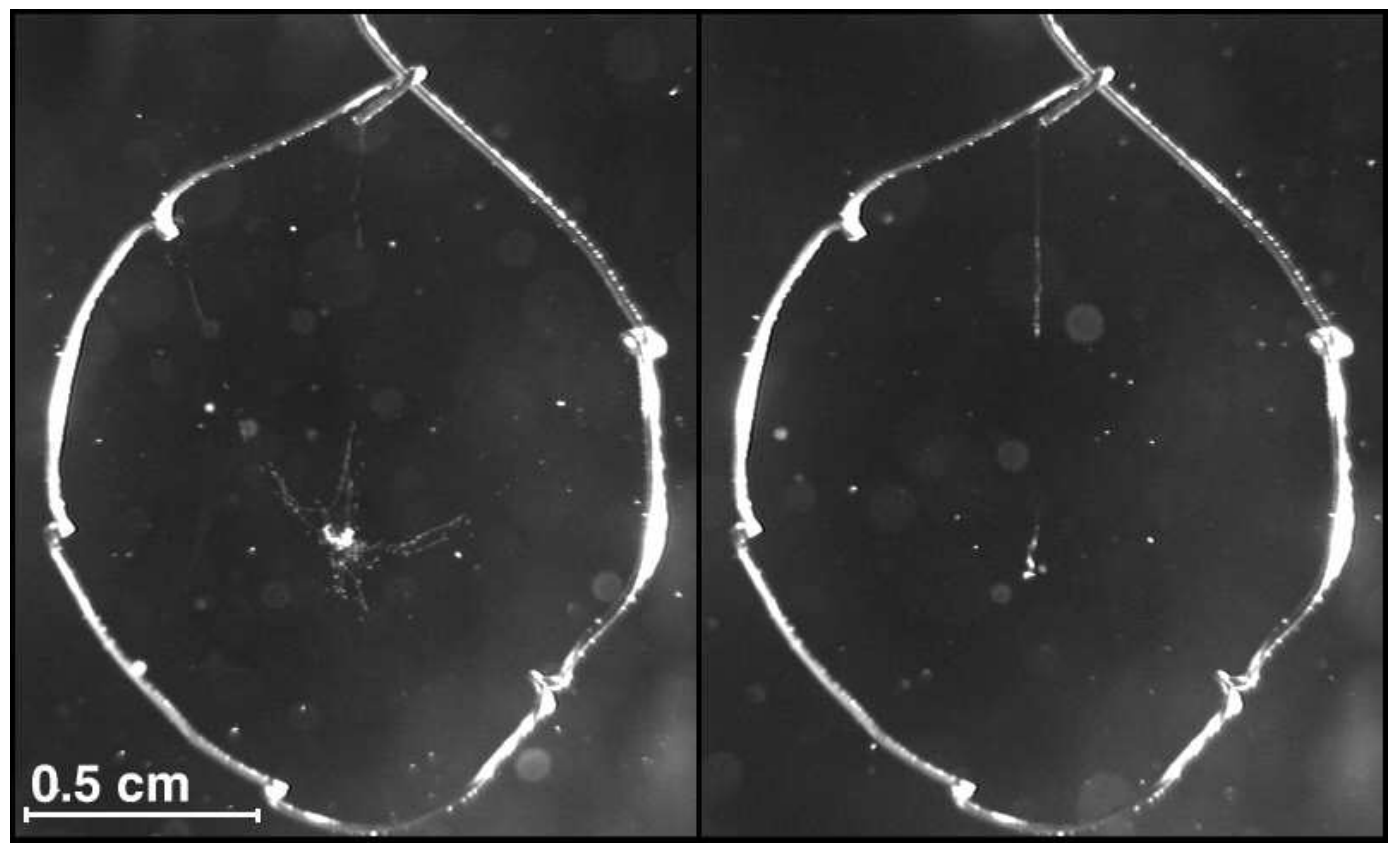

Abbildung 7.11: Versiegen von Blasenquellen. Bildabstand $>5 \mathrm{~s}, \mathrm{Be}-$ lichtungszeit $16 \mathrm{~ms}$.

Erliegen gekommen, eine große Blase oder ein Blasenkonglomerat bildet sich in der Mitte (Es handelt sich hierbei nicht um einen stabilen Cluster wie in anderen Konfigurationen). Diese wird nach kurzer Zeit aus dem Zentrum gezogen, allerdings nicht nach oben sondern reproduzierbar nach unten in Richtung des Schallwandlers oder dorthin, wo der Drahtring am nächsten ist.

In Abbildung 7.13 ist der Moment der Zerstörung der großen Blase dargestellt. In Bild 1 und 2 bewegt sich die Blase, einen Schweif von Blasen hinter sich lassend, nach rechts unten, aus der Länge des Streaks lässt sich eine Translationsgeschwindigkeit von etwa $0.2 \mathrm{~m} / \mathrm{s}$ abschätzen. In Bild 3 trifft die Blase auf den Draht, sofort beginnen vorher ruhende Blasen in die Mitte zu laufen. 


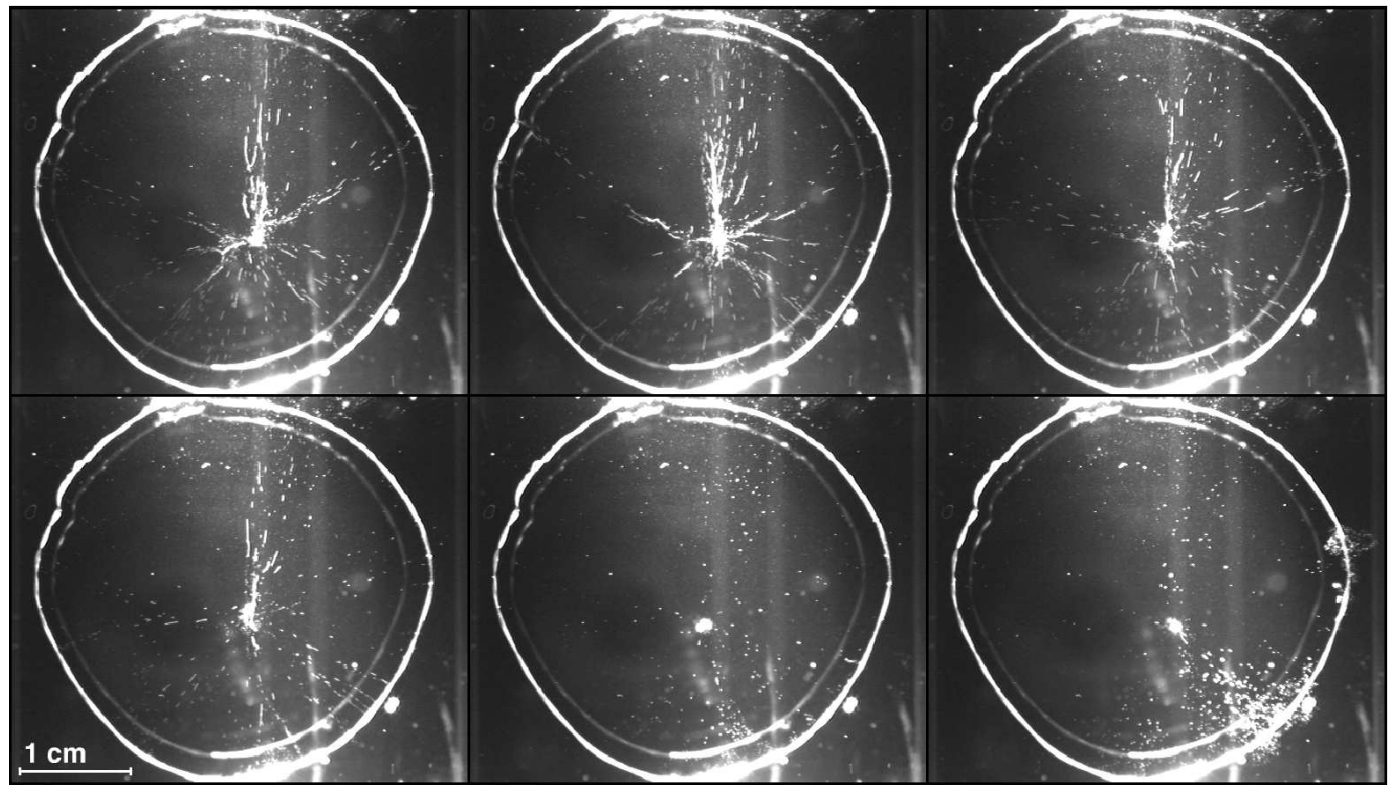

Abbildung 7.12: Instabile akustische Lichtenbergfigur. Bildabstand $2 \mathrm{~s}$, Belichtungszeit $16 \mathrm{~ms}$.

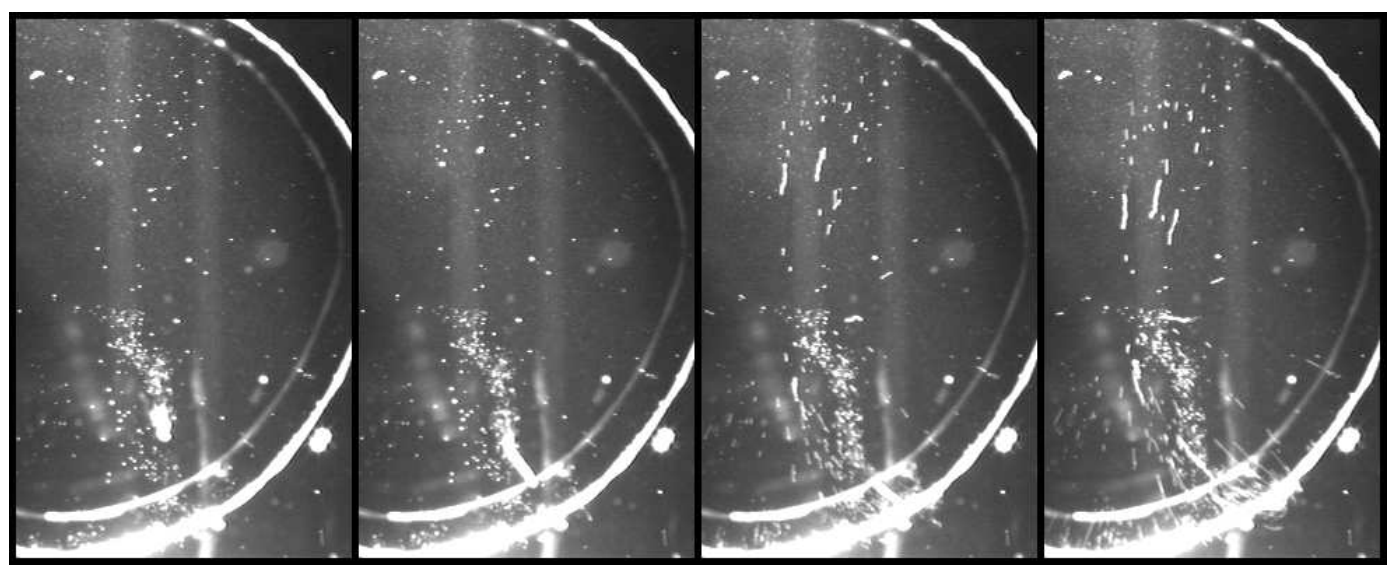

Abbildung 7.13: Zerstörung der zentralen Blase. Belichtungszeit und Bildabstand $16.7 \mathrm{~ms}$. 


\section{Kapitel 8}

\section{Jellyfish-Strukturen}

\subsection{Die Jellyfish-Struktur}

Im Ultraschallreaktor (siehe Anhang A.4) bilden sich Doppellagenstrukturen aus, die aufgrund ihrer optischen Ähnlichkeit mit Quallen im folgenden „Jellyfish-Strukturen“ genannt werden. Während in der Nähe des Randes vergleichsweise wenig Blasen zu sehen sind, bilden sie sich im Zentrum des Reaktors. Sie haben eine Ausdehnung von etwa $1 \mathrm{~cm}$. Bereits bei unterster Leistungsstufe des Reaktors können diese Strukturen beobachtet werden, die bis zur maximalen Leistungsstufe existieren.

\subsubsection{Durchführung}

Die Kavitationsstrukturen wurden mit Hochgeschwindigkeitskinematographie für verschiedene Leistungsstufen des Resonators aufgezeichnet.

\subsubsection{Ergebnisse}

\section{Orientierung im Schallfeld}

In Abbildung 8.1 sind typische Streulichtaufnahmen für die Blasenverteilung wiedergegeben. Auffällig ist die ausgeprägte Doppellagenstruktur sowie die Anordnung in verschiedenen Ebenen. Von oben betrachtet hat jede einzelne der beiden Lagen eine filamentartige Struktur. Diese ist in etwa rotationssymmetrisch. Oft befindet sich eine große Blase im Zentrum des Jellyfish. 


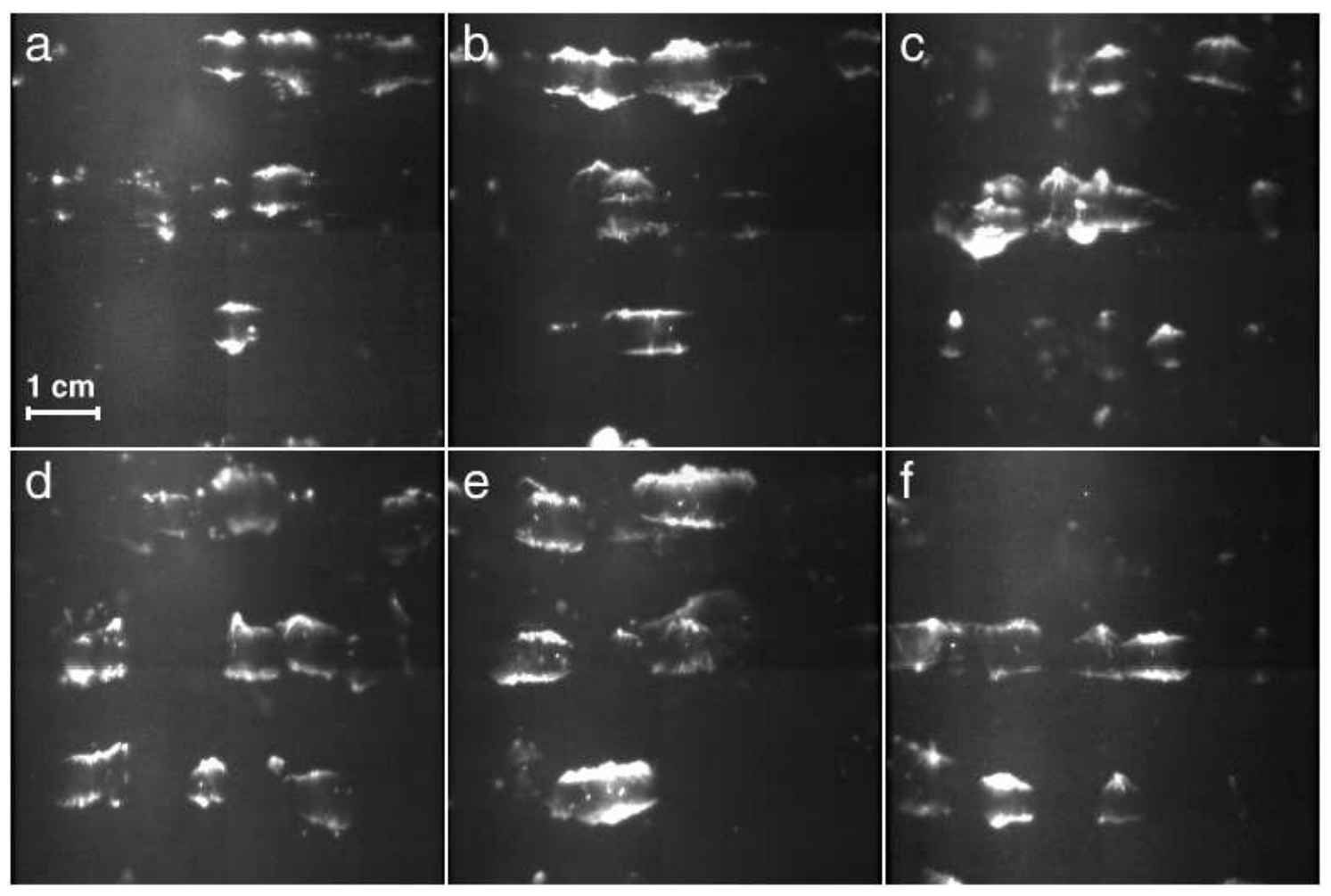

Abbildung 8.1: Die Jellyfish-Struktur im Ultraschallreaktor, Seitenansicht. Streulichtaufnahmen bei verschiedenen Leistungsstufen. (a: $15 \%$, b: $20 \%$, c: $50 \%$, d: $60 \%$, e: $80 \%$, f: $100 \%$ ).

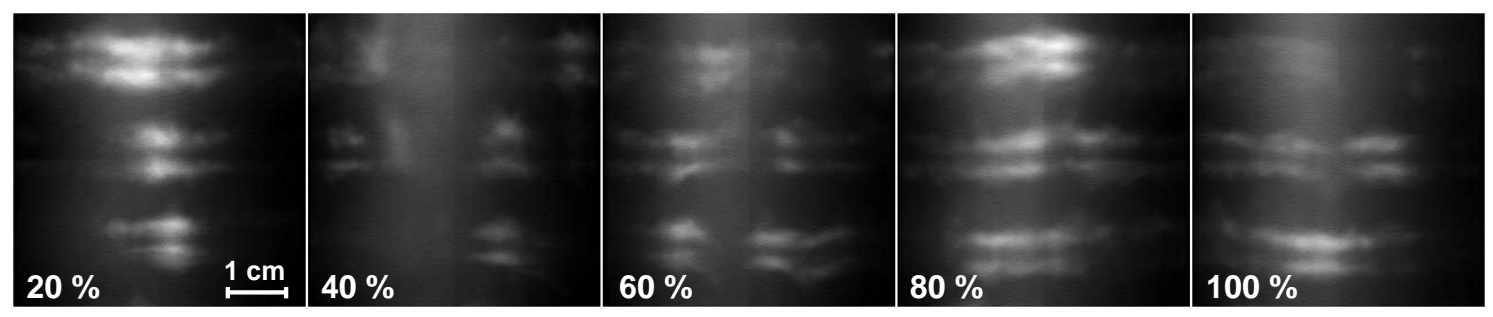

Abbildung 8.2: Über etwa 2 Sekunden gemittelte Blasenpositionen im Ultraschallreaktor bei verschiedenen Leistungsstufen. Während die vertikale Lage der Blasenstrukturen bei allen Aufnahmen gleich ist, ist die horizontale Position weniger scharf. 


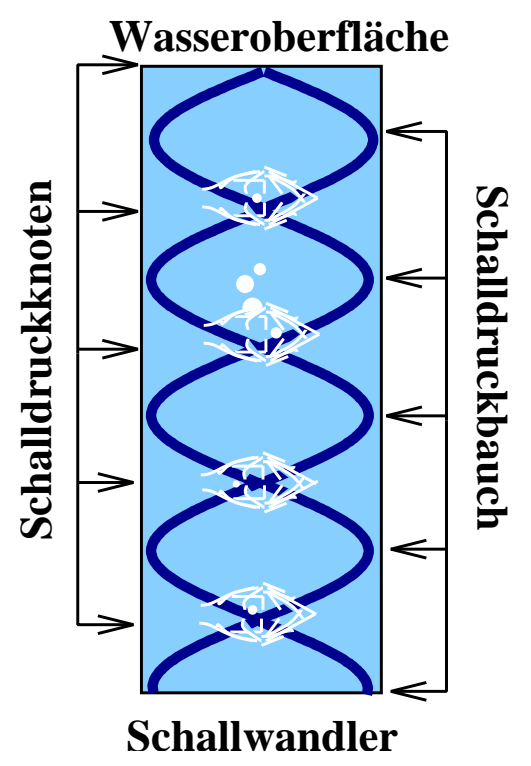

Abbildung 8.3: Lage der Jellyfish-Strukturen im Schallfeld, schematisch dargestellt.

Die einzelnen Strukturen können sich in horizontaler Richtung bewegen, bis zum Verschmelzen benachbarter Figuren. Auch zwischen den Doppellagen selber besteht eine enge Verbindung. Einzelne Lagen treten in der Regel nur direkt unter der Oberfläche auf (siehe Abschnitt 8.2). In vertikaler Richtung jedoch sind die Strukturen in streng getrennten Ebenen organisiert. Zwischen diesen Ebenen scheint die Flüssigkeit weitgehend von Blasen entvölkert zu sein. Abbildung 8.2 gibt die über 2000 Bilder (etwa 2 s) gemittelte Blasenverteilung im Ultraschallreaktor bei verschiedenen Leistungsstufen wieder.

Helle Bereiche kennzeichnen Orte hoher Aufenthaltswahrscheinlichkeit von Blasen. Die Blasen halten sich vornehmlich in drei horizontalen Ebenen auf, die jeweils in zwei Lagen aufspalten. Die vertikale Position der Ebenen ist unabhängig von der Schallintensität. Die Ebenen weisen auch eine vertikale Struktur auf, diese ist allerdings von Film zu Film verschieden und es zeichnet sich keine eindeutige Tendenz ab. Die Mitte des Bildausschnitts (etwa das Zentrum des Reaktors) scheint aber ein Ort sehr hoher (20\%) oder alternativ sehr niedriger $(60 \%)$ Blasenaufenthaltswahrscheinlichkeit zu sein. Die vertikalen Abstände zwischen den Strukturen bleiben nahezu konstant und entsprechen etwa der halben Wellenlänge der Schallwelle, auch wenn das gesamte Blasenfeld sich auf und ab bewegen kann. Während der Schwerpunkt der Doppellagenstruktur in den vertikalen Schalldruckminima liegt, sind die Schalldruckmaxima in den Ebenen zwischen den Strukturen lokalisiert. Die vertikale Anordnung der Jellyfish-Strukturen im Schallfeld ist in Abbildung 8.3 schematisch dargestellt. 
Ähnlich wie Streamerstrukturen bei hohen Schalldruckamplituden sind hier die Orte hohen Schalldrucks entvölkert, die Struktur ist eher um den Schalldruckknoten als um den Schalldruckbauch orientiert.

Da eine Knotenlinie zwischen den Lagen der Struktur liegt, schwingen sie gegenphasig. Dies bestätigt eine phasenaufgelöste Ultrahochgeschwindigkeitsaufnahme der Jellyfish-Struktur. In Abbildung 8.4 ist deutlich das um eine halbe Schwingungperiode verschobene Oszillationsverhalten der beiden Doppellagen zu erkennen.

Auch in diesem Reaktor wird meist eine niederfrequente Schwerewelle angeregt, die ein Auf- und Abschwappen der Flüssigkeit bewirkt. Die Ebenen der JellyfishStrukturen folgen dabei der Flüssigkeit. Diese Oberflächenwelle scheint auch hier einen verstärkenden Effekt auf die Kavitationsbildung zu haben. Die Strukturen wirken in der Aufwärtsbewegung ausgedehnter und mit mehr Blasen bevölkert. Die Entwicklung ist exemplarisch in Abbildung 8.5 dargestellt.

Zusätzlich ist in der Sequenz im unteren Drittel eine auf der Wand sitzende relativ große Blase zu sehen. Diese führt eine den Kavitationsstrukturen entgegengesetzte Bewegung aus, da sich zwischen ihnen eine Knotenlinie der Schwerewelle befindet.

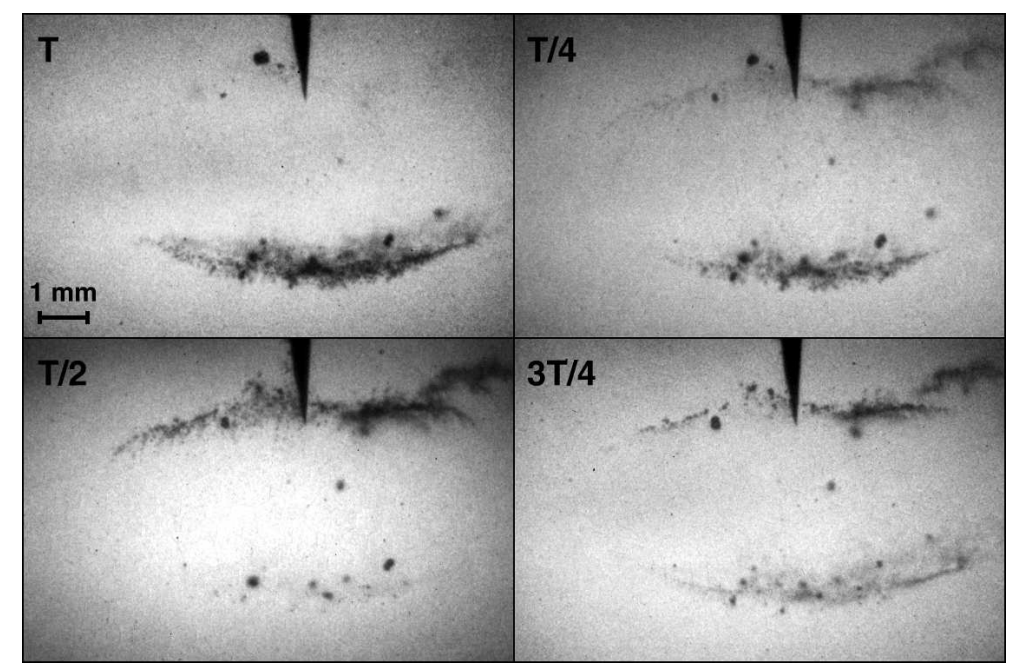

Abbildung 8.4: Phasenaufgelöste Aufnahme einer Jellyfish-Struktur. Der Bildabstand beträgt $6.25 \mu \mathrm{s}$. Aufnahme von R. Mettin. 


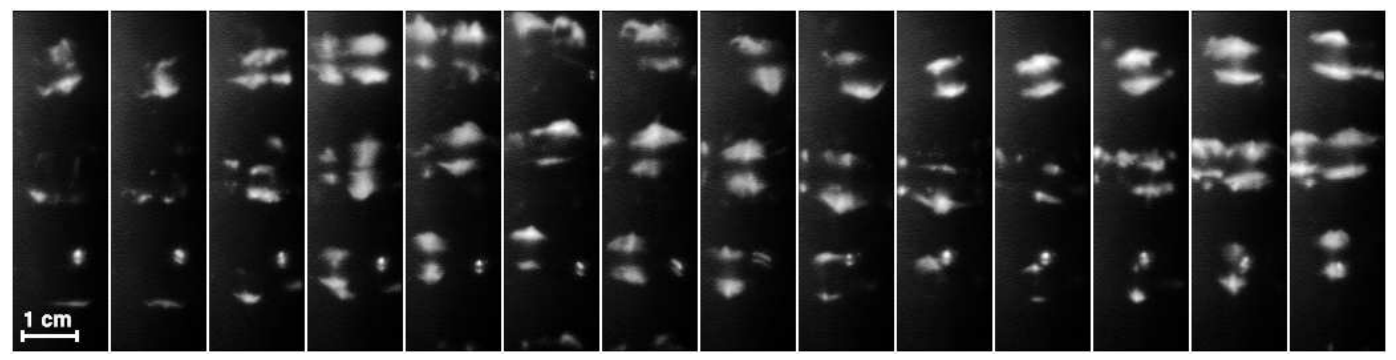

Abbildung 8.5: Die Jellyfish-Strukturen bewegen sich mit der niederfrequenten Oberflächenwelle auf und ab. Um die hochfrequente Amplitudenmodulation herauszufiltern, wurde das Bild über 45 Einzelbilder gemittelt. Der Bildabstand beträgt etwa $40 \mathrm{~ms}$. Der Reaktor wurde bei $15 \%$ betrieben.

Entstehung, Koaleszenz und Abspaltung Die Jellyfishstrukturen entstehen aus Blasenclustern, die sich spontan in der blasenarmen Zone bilden und von dort zu den Ebenen hoher Blasenaufenthaltswahrscheinlichkeit laufen. In Abbildung 8.6 ist exemplarisch eine solche Entstehung dargestellt. Der Bildabstand beträgt etwa $2.7 \mathrm{~ms}$.

Der entstandene Blasencluster läuft als recht kompakte Struktur nach unten. Dabei beginnt er, sich in die typische Doppellagenstruktur aufzuspalten, die sich im Folgenden weiter stabilisiert. Die Aufnahme wurde bei $50 \%$ Leistung gemacht, entsprechende Strukturbildungsprozesse finden sich bei allen Leistungsstufen.

Typische Lebensdauern dieser Strukturen zu bestimmen, ist nahezu unmöglich. Oft sind Jellyfish-Strukturen nicht mehr sichtbar, schwingen dann aber in der Aufwärtsbewegung der Schwerewelle wieder auf. Zudem finden sowohl Vereinigungsprozesse von sich bildenden Blasenclustern mit bereits bestehenden Strukturen als auch Vereinigungs- und Abspaltungsprozesse zwischen horizontal benachbarten Jellyfish-Strukturen statt. In Abbildung 8.7 ist eine Vereinigung von einzelnen Strukturen abgebildet.

$\mathrm{Zu}$ Beginn scheinen die beiden rechten Strukturen zusammenzulaufen, dann jedoch vereinigen sich zuerst die linke mit der mittleren Kavitationsfigur. Die vereinigte Jellyfishstruktur schrumpft in horizontaler Richtung, bis sie etwa die gleiche Größe wie die rechte Figur hat, um sich dann mit dieser zu vereinigen. 


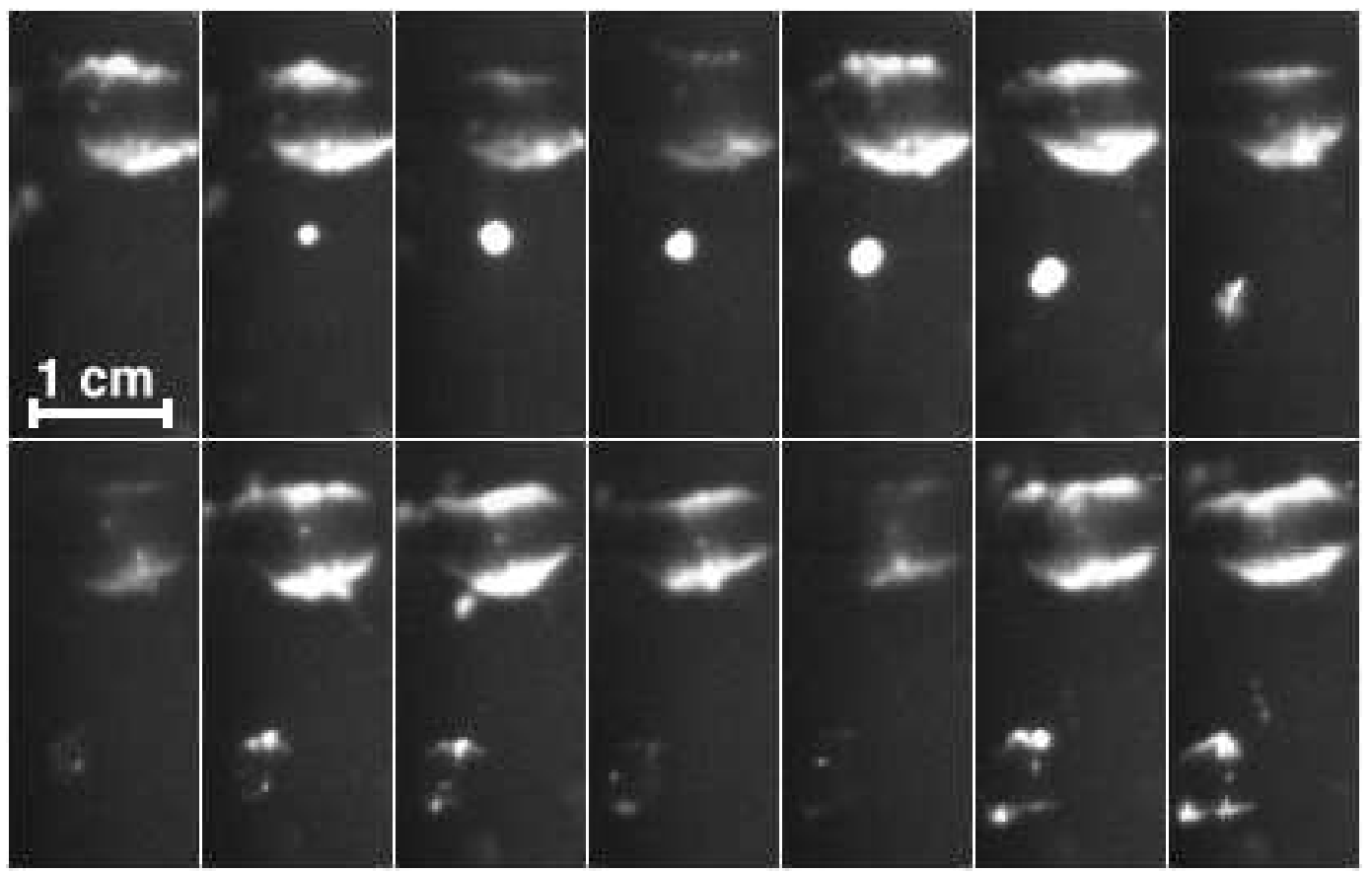

Abbildung 8.6: Entstehung einer Jellyfish-Struktur aus einem spontan entstehende Blasencluster. Der Bildabstand beträgt etwa $2.7 \mathrm{~ms}$. Die HellDunkel-Variation der Blasenstrukturen ist durch die $100 \mathrm{~Hz}$ Amplitudenmodulation bedingt. Der Reaktor wurde bei $50 \%$ betrieben.

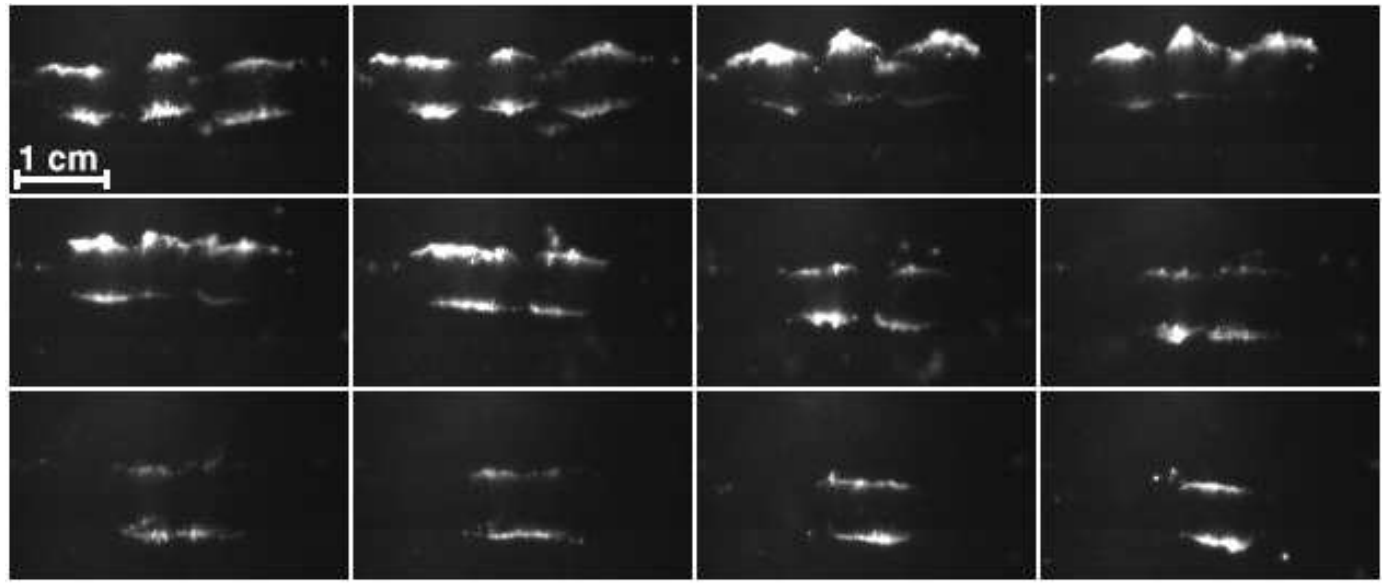

Abbildung 8.7: Vereinigung von drei Jellyfish-Strukturen. Der Bildabstand beträgt $21.4 \mathrm{~ms}$. Der Reaktor wurde bei $15 \%$ betrieben. 


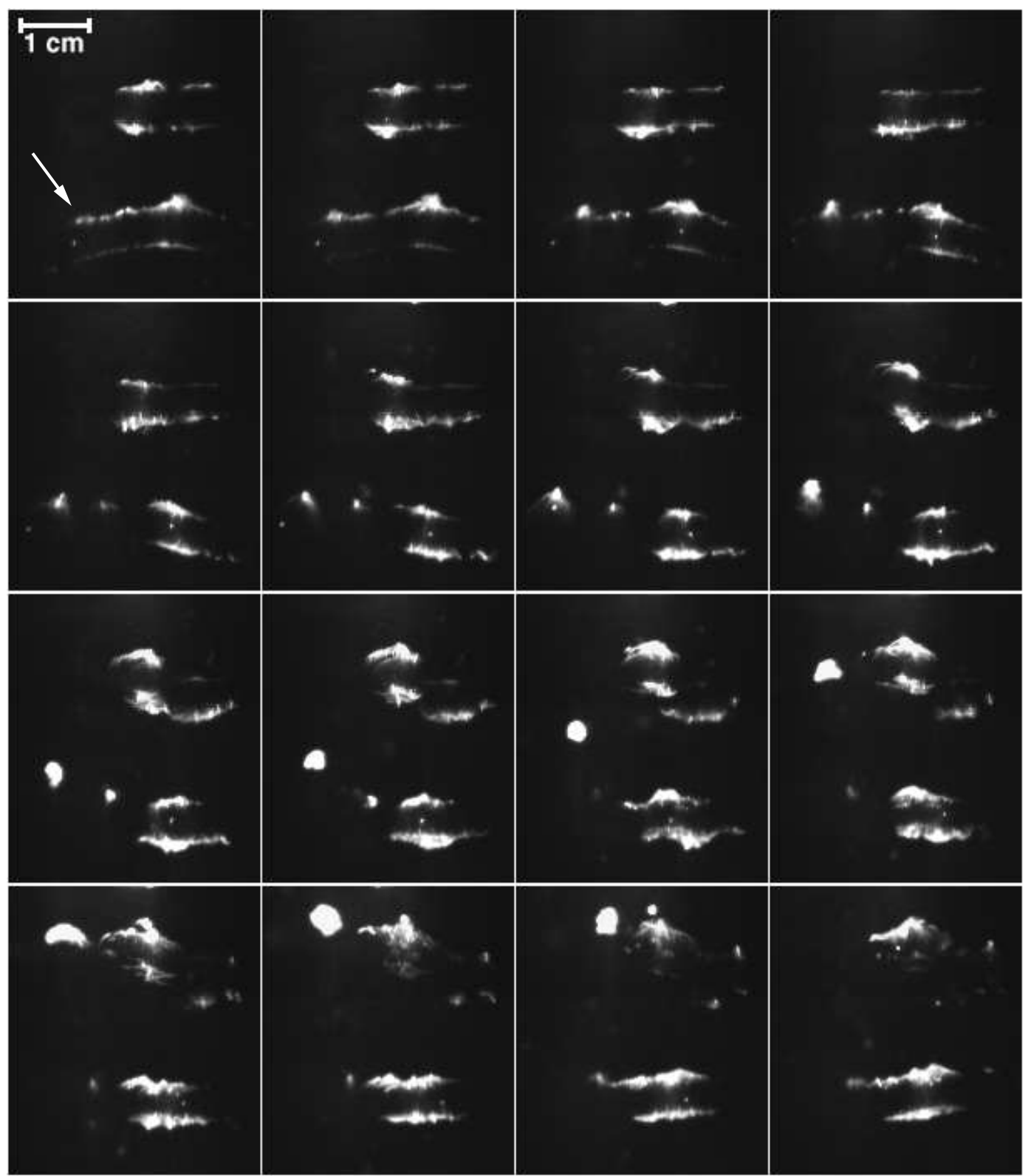

Abbildung 8.8: Fragmentations- und Vereinigungsprozesse von Jellyfish-Strukturen. Der Bildabstand beträgt 10.7 ms. Der Reaktor wurde bei $20 \%$ betrieben. 
Es können auch Fragmentationsprozesse beobachtet werden. In Abbildung 8.8 spalten sich zwei Fragmente (Pfeil) von der unteren Blasenstruktur ab.

Der kleinere, der Jellyfish-Struktur nähere Teil vereinigt sich wieder mit der Struktur. Der linke Teil bildet in einem zum Entstehungsprozess umgekehrten Verlauf einen Cluster. Dieser steigt auf und läuft in die sich eine Ebene darüber befindliche Kavitationsfigur. Im vorletzten Bild ist ein weiterer kleiner Cluster oberhalb der Jellyfish-Struktur zu sehen. Die Dynamik dieses Fragments ist in Abbildung 8.9 in höherer Zeitauflösung dargestellt.

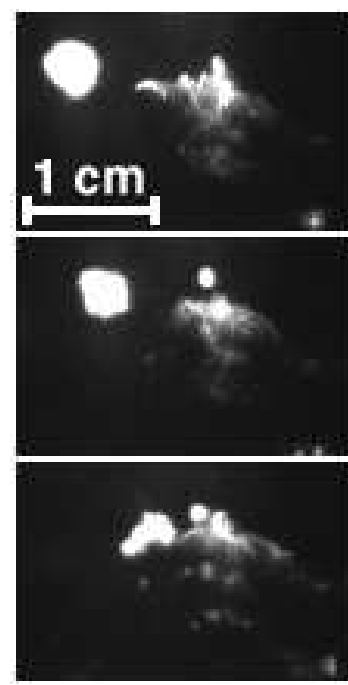

Abbildung 8.9: Fragmentations-
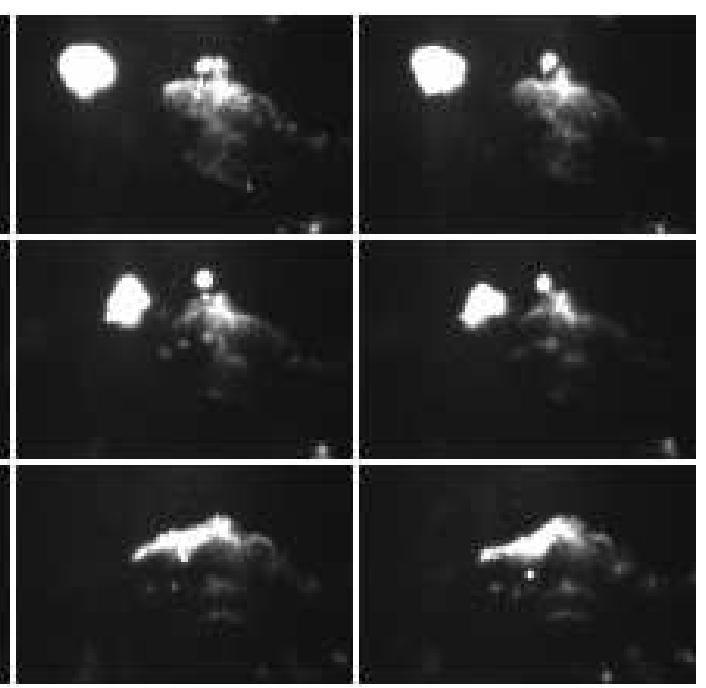

und Vereinigungsprozesse von Jellyfish-Strukturen. Ausschnitt aus Abb. 8.8. Der Bildabstand beträgt $1.7 \mathrm{~ms}$. Der Reaktor wurde bei $20 \%$ betrieben.

Hier sehen wir die Bildung von zuerst drei kleineren Blasen oder Blasenclustern. Aus diesen bildet sich dann ein Cluster, der sich abspaltet. Zeitgleich mit dem großen Cluster vereinigt er sich wieder mit der Jellyfish-Struktur. In den letzten zwei Bildern beobachten wir die Abspaltung einer großen Blase in Richtung des Strukturzentrums. Es bildet sich eine „Perle“, wie sie häufig in JellyfishStrukturen zu beobachten ist.

Neben den sich von bereits existierenden Kavitationsstrukturen ablösenden Blasenclustern können spontan entstehende Cluster beobachtet werden, die aus dem Bereich des Schalldruckbauchs kommen. Diese transienten Cluster entsprechen denen von Crum 1972 beschriebenen transient cavities [20]. Diese entstehen ebenfalls spontan im Schalldruckbauch und zeigen eine vergleichbare Struktur. 


\subsubsection{Transiente Jellyfishstrukturen}

Um die am Ultraschallreaktor gefundenen Ergebnisse verallgemeinern zu können, wird ein Kontrollexperiment im Plexiglasquader durchgeführt (siehe Anhang A.2). Um horizontale Knotenflächen zu erhalten, wird der Resonator bei einer Frequenz von $43.3 \mathrm{kHz}$ und bei maximaler Spannung $\left(U_{F G}=20 \mathrm{Vpp}\right.$, $U_{p}=202 \mathrm{Vpp}$ ) betrieben. Aber erst durch die (spontane) Anregung der tieffrequenten Schwerewellen wird die Kavitationsschwelle gelegentlich überschritten. Ebenso wie im Ultraschallreaktor entstehen spontan Cluster im Schalldruckbauch und am Boden, allerdings nur vereinzelt. Diese verlassen dann mit hoher Geschwindigkeit den Schalldruckbauch und bilden die typischen Doppellagenstrukturen. Wenn kein weiterer Cluster erzeugt wird, wird die Jellyfishstruktur nach wenigen Millisekunden dünner und verschwindet. Auch dies ist analog zum Verhalten der Jellyfish-Strukturen im Ultraschallreaktor, mit dem Unterschied, dass die Strukturen dort durch die Schwerewelle lediglich moduliert werden und nicht völlig verschwinden. In Abbildung 8.10 ist ein solcher Zyklus von Entstehung aus einem im Volumen entstandenen Cluster (a,b), Vereinigung mit einem neu entstehenden Cluster (d) und dem langsamen Verschwinden (e) wiedergegeben. Die letzte Sequenz (f) zeigt die Entstehung einer neuen Jellyfish-Struktur, die sich aus einem am Resonatorboden entstehenden Cluster bildet. Aufgrund der unterschiedlichen Zeitskalen, auf der sich die Entwicklung vollzieht, wurde der Bildabstand nur innerhalb einer Teilsequenz konstant gehalten. Der Balken unter den Filmsequenzen gibt den Zeitraum an, den die jeweilige Teilsequenz umfasst. Das Kontrollexperiment zeigt, dass die Jellyfish-Struktur eine Grundform der Kavitationsstrukturen ist. Sie entsteht aus der Clusterstruktur und richtet sich an Knotenflächen aus.

Da Schallwandler in Ultraschallwannen meist am Boden angebracht sind und eine ausgeprägte Richtcharakteristik zeigen, liegen die Jellyfishstrukturen im Allgemeinen in horizontaler Anordnung. Ein Experiment in einer sehr großen Reinigungswanne mit einem Volumen von etwa $1 \mathrm{~m}^{3}$ (Testwanne der Firma Elma), bei der auch Schallwandler an den Seitenwänden angebracht sind, bestätigt, dass sich bei Reflexion an einer schallweichen Wand (hier simuliert durch eine kleinere ungefüllte Glaswanne) Jellyfish-Strukturen auch entsprechend den entstehenden Knotenlinien vertikal anordnen können. 


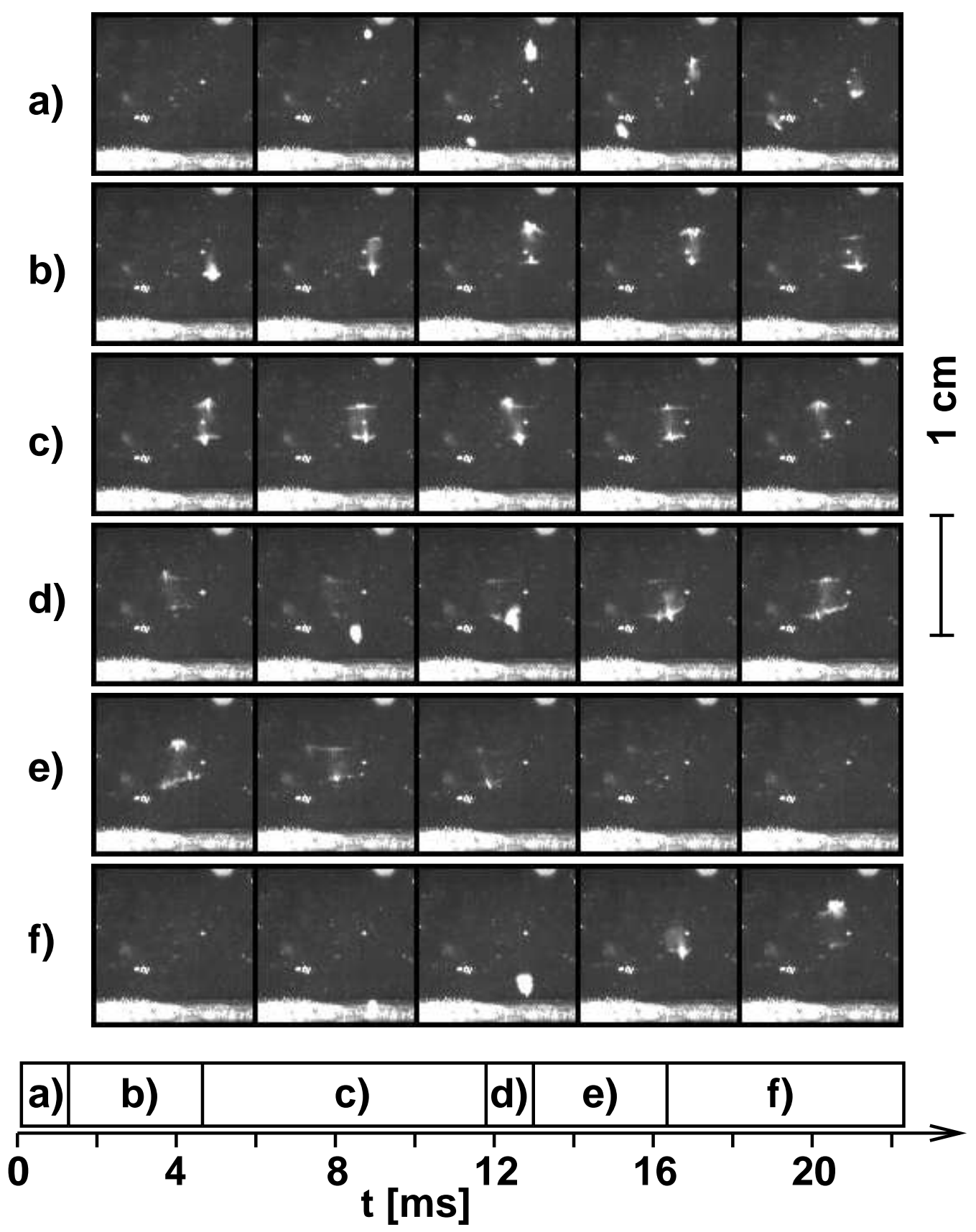

Abbildung 8.10: Lebenszyklus einer transienten Jellyfish-Struktur im Plexiglasquader. Der Bildabstand jeder Sequenz ist der intrinsischen Zeit der dargestellten Ereignisse angepasst. Das Balkendiagramm unter den Filmsequenzen gibt den Zeitraum an, den die jeweilige Sequenz umfasst. 


\subsection{Die Starfish-Struktur - Blasenstruktur nahe der Wasseroberfläche}

In größeren Kavitationsbecken entstehen oft nach einer Weile Kavitationsstrukturen an der Wasseroberfläche. Sie richten sich parallel zur Oberfläche aus und bilden eine verzweigte, sternförmige Struktur. Die Kavitationsstruktur zeigt viele Eigenschaften einer Jellyfish-Struktur, deren obere Lage fehlt, also quasi an der Wasseroberfläche gespiegelt wird. Diese im weiteren Starfishstruktur benannte Kavitationsstruktur konnte bisher nur in Anwesenheit der tieffrequenten Schwerewelle beobachtet werden und befindet sich in deren Auslenkungsmaximum. Sie ist aber in ihrer Lebensdauer nicht an deren Schwingungsfrequenz gebunden. Entsteht eine Starfish-Struktur, so ist die Wasseroberfläche dort deutlich angehoben. In Abbildung 8.11 ist die Aufsicht (a) und die Seitenansicht (b) einer solchen Struktur abgebildet. Insbesondere in der Plexiglaswanne entsteht oft eine langlebige ausgedehnte Starfish-Struktur. Charakteristisch ist, dass der Rand der Struktur nach oben gebogen scheint. Das diffuse Zentrum erinnert an das Halo im Zentrum der akustischen Lichtenbergfigur. Im kleineren Plexiglasquader konnte diese Struktur bisher nicht reproduziert werden. Während JellyfishStrukturen sich meist aus spontanen Clustern bilden und dabei Fragmentationsund Koaleszenzprozessen ausgesetzt sind, ist die Starfish-Struktur innerhalb ihrer Lebensdauer vergleichsweise stabil. In der Plexiglaswanne ist sie, einmal entstanden, bis zur Veränderung von Frequenz oder Anregungsamplitude präsent.
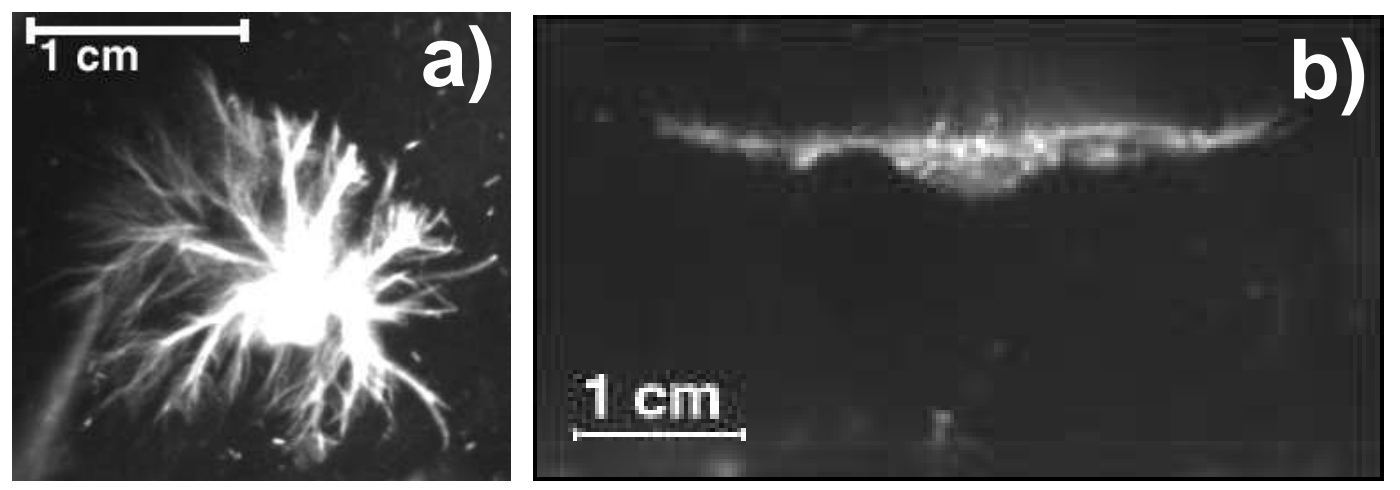

Abbildung 8.11: Oben: Langzeitbelichtung einer Starfish-Struktur im Ultraschallreaktor, Aufsicht (a). Seitenansicht einer Starfish-Struktur in der Ultraschallwanne (b). 


\subsubsection{Bestimmung der räumlichen Dimension einer Starfish- Struktur}

Die selbstähnliche Verzweigungsstruktur in der Langzeitbelichtung dieser Kavitationsstruktur motiviert den Versuch, die fraktale Dimension des Systems zu bestimmen.

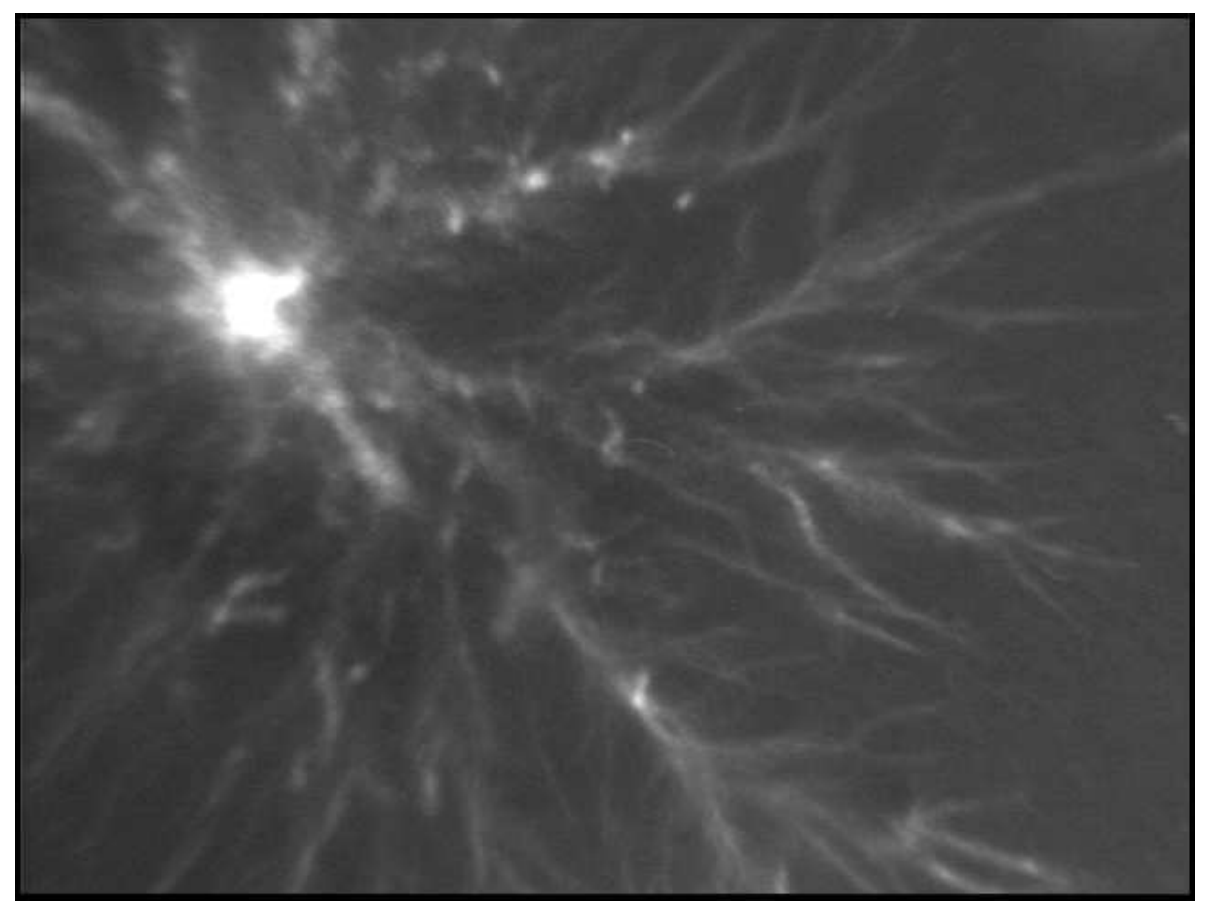

Abbildung 8.12: Oben: Langzeitbelichtung einer Starfish-Struktur im Ultraschallreaktor, Aufsicht, Detailaufnahme.

Basis ist die Korrelationsdimension nach Grassberger und Procaccia [40]

$$
D_{\text {corr }}=\lim _{r \rightarrow 0} \frac{\log C(r)}{\log r}
$$

mit

$C(r)=\lim _{n_{R} \rightarrow \infty} \lim _{n_{D} \rightarrow \infty} \frac{\sum_{k=1}^{n R} N_{x_{k}}(r)}{n_{R} n_{D}}:$ Korrelationssumme, $n_{R}:$ Anzahl der Referenzpunkte,

$n_{D}$ : Anzahl der Datenpunkte,

$N_{x_{k}}$ : Anzahl der Punkte, die innerhalb eines Radius $\mathrm{r}$ um den Referenzpunkt $x_{k}$ liegen.

In einem realen System mit endlicher Ausdehnung und Auflösung ist die Selbstähnlichkeit nur in einem begrenzten Raumbereich gegeben, dieser Bereich 
bestimmt auch das Radiusintervall, in dem die Dimensionsbestimmung sinnvoll ist. Neben der Starfish-Struktur wird zum Vergleich die Dimension einer akustischen Lichtenbergfigur, wie sie typischerweise im Plexiglasquader entsteht, bestimmt.
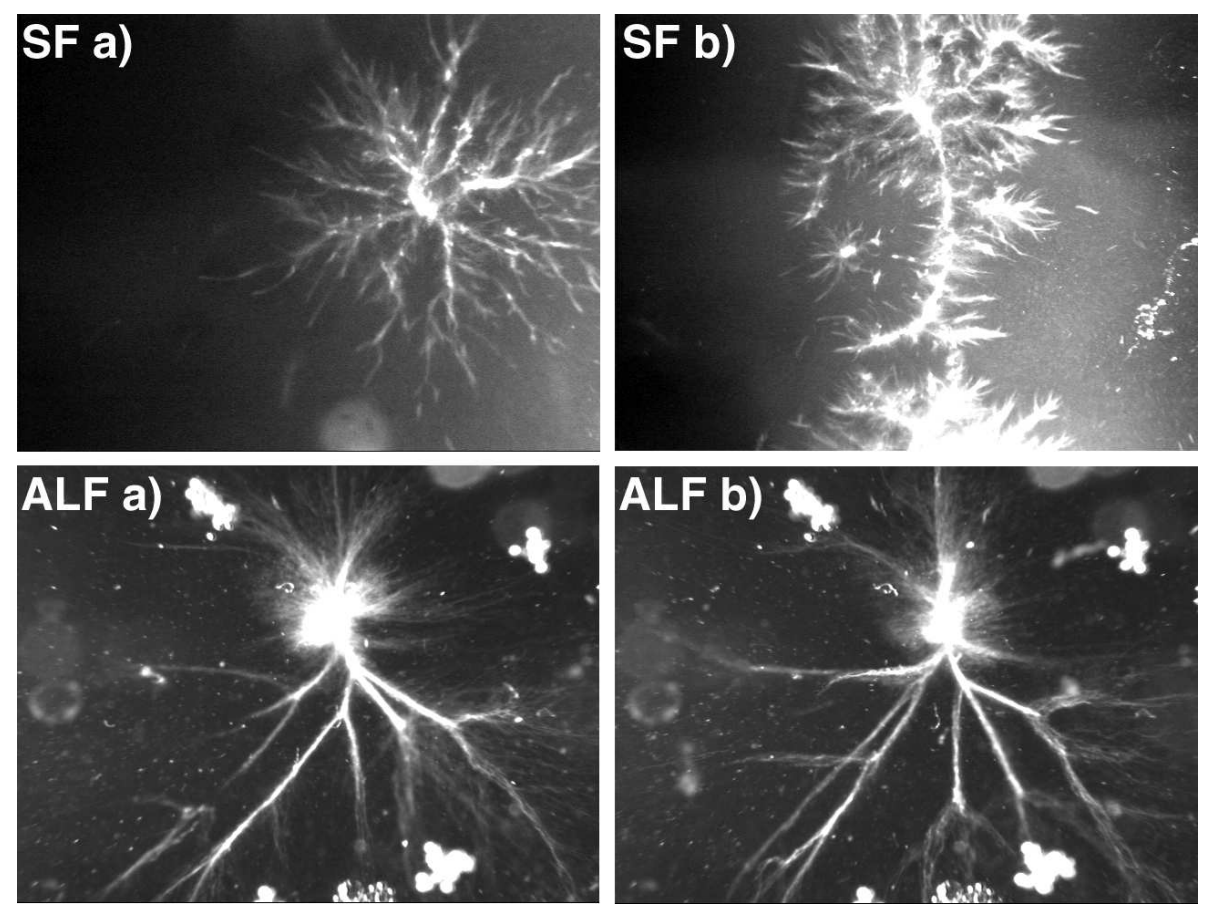

Abbildung 8.13: Langzeitbelichtete Aufnahmen von StarfishStrukturen (SF) und akustischen Lichtenbergfiguren (ALF). Bildgröße: $480 \times 640$ pxl.

Dazu werden zweidimensionale Langzeitaufnahmen der Kavitationsstrukturen angefertigt. In Abbildung 8.13 sind die verwendeten Aufnahmen wiedergegeben. Die Starfish-Strukturen (SF) zeigen eine sehr feine Verzweigungsstruktur, während die Filamente der akustischen Lichtenbergfigur (ALF) in der Nähe des Zentrums verzweigungsarm werden. Zur Datenanalyse werden die Bilder bandpassgefiltert, binarisiert und in normalisierte Koordinaten transformiert. In Abbildung 8.14 sind die auf diese Weise ermittelten Punkte dargestellt. Die dicker erscheinenden Punkte in den akustischen Lichtenbergfiguren resultieren aus Anhäufungen von Punkten. Die Korrelationssumme von 1000 Referenzpunkten wurde mit Hilfe des tstool, ein Softwarepaket zur nichtlinearen Zeitreihenanalyse [67] bestimmt. Das Skalierungsverhalten ist in Abbildung 8.15 (a) dargestellt.

Für die Starfish-Struktur ergibt sich eine etwas steilere Steigung als für die akustischen Lichtenbergfiguren, es zeigen sich jedoch über den Radiusbereich signifikante Änderungen im Skalierungsverhalten. Zur besseren Bewertung ist in Ab- 

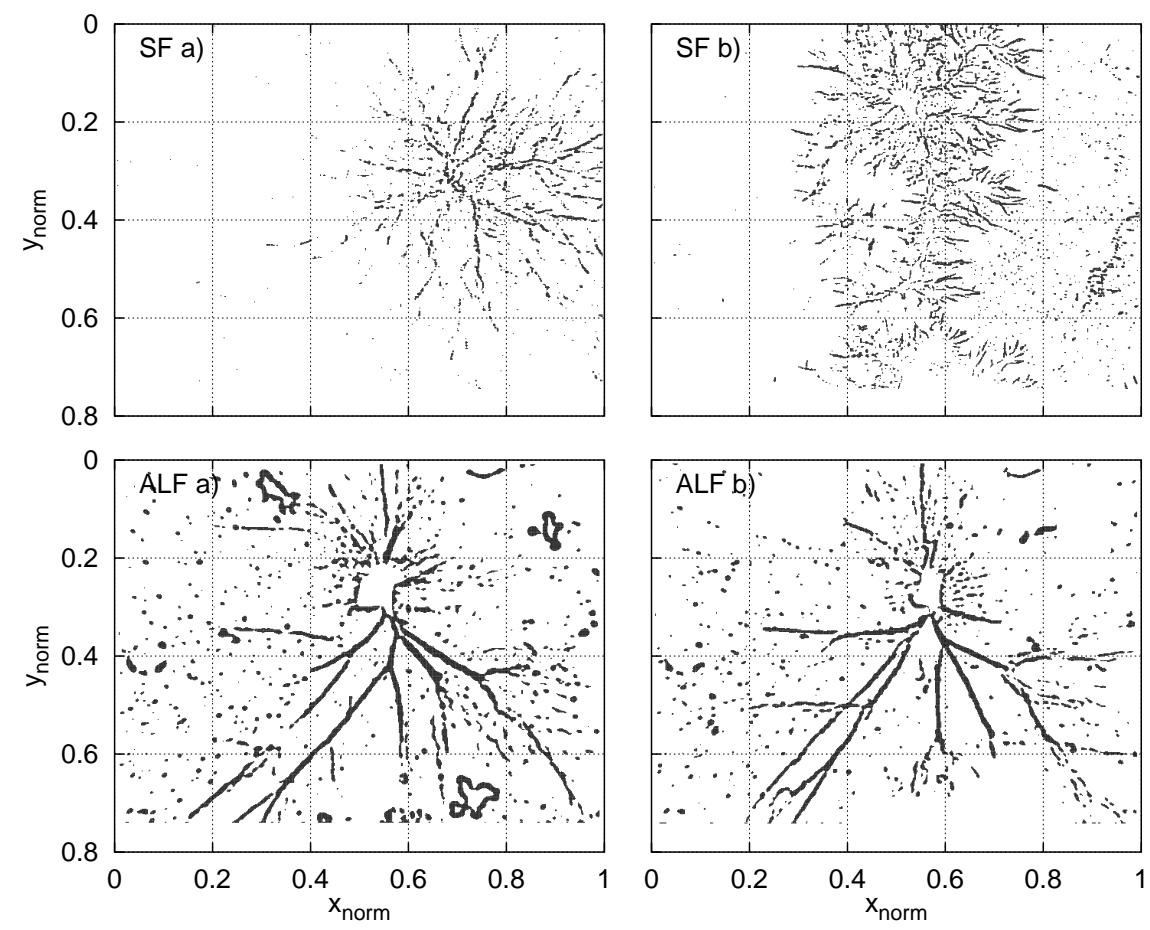

Abbildung 8.14: Extrahierte Datenpunkte der Aufnahmen 8.13 in normalisierten Koordinaten.

bildung 8.15 die Steigung $D_{\text {corr }}=\log C(r) / \log r$ linear gegen den Radius in Bildpunkten aufgetragen. Abbildung (b) zeigt den gesamten Parameterbereich, in Graph (c) ist noch einmal der Ausschnitt des Pixelbereiches dargestellt, der zur Bestimmung der Dimension verwendet wird. Die senkrechten Striche geben die ermittelte fraktale Dimension für die verschiedenen Abbildungen wieder. Unterhalb des Radius von etwa 3 Pixel schwankt die Korrelationsdimension sehr stark, dieser Bereich liegt in der Größenordnung der Filamentbreite, dort sind Verzweigungsstrukturen noch nicht aufgelöst. Für die Starfish-Strukturen ergibt sich insgesamt eine höhere fraktale Dimension von 1.80 \pm 0.09 , für die akustische Lichtenbergfigur eine Korrelationsdimension von $1.70 \pm 0.07$. Auch bei leichter Änderung der Filterparameter der Bildbearbeitung liegt die ermittelte fraktale Dimension im Fehlerbereich der angegebenen Werte. Insgesamt stellt sich das Problem, dass bei einer Bildgröße von 460 x 640 Bildpunkten der Radiusbereich nur über einen Bereich von etwas mehr als 2 Größenordnungen aufgelöst werden kann, der nach oben durch die Größe der Kavitationsstruktur, nach unten durch die Blasengröße begrenzt ist. Bei einem Blasenradius von etwa $10 \mu \mathrm{m}$ und einer räumlichen Ausdehnung der Struktur von etwa $1 \mathrm{~cm}$ kann das Skalierungsverhalten prinzipiell über maximal 3 Größenordnungen verfolgt werden. Dies erfordert 

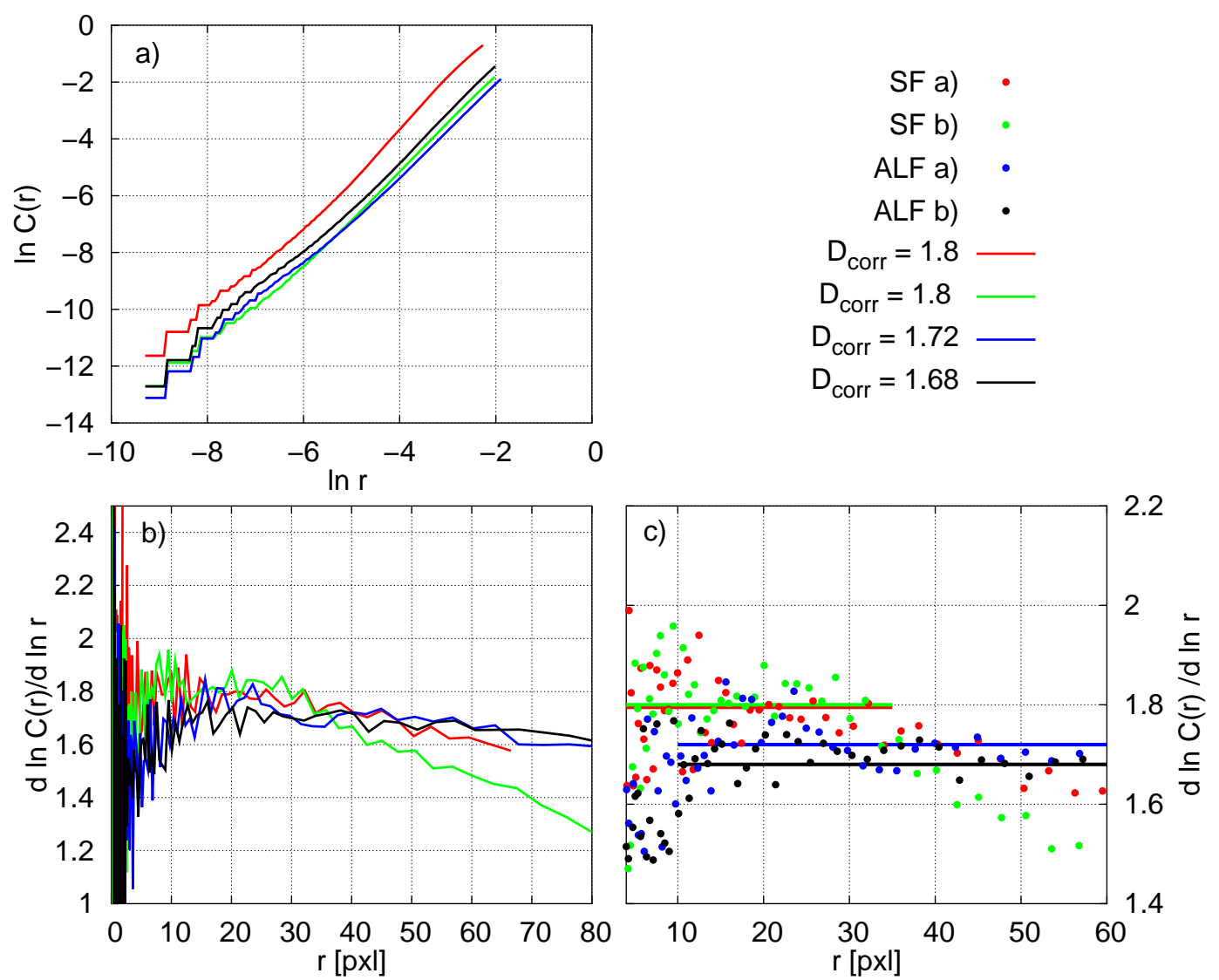

Abbildung 8.15: Doppelt logarithmische Auftragung der mittleren Korrelationssumme gegen den Radius (a) sowie die Steigung in Abhängigkeit vom Radius in Bildpunkten (b,c).

allerdings einen recht hohen experimentellen Aufwand, da die Belichtungszeit gerade so abgepasst werden muss, dass bereits zusammenhängende Streamerarme erkennbar sind, die einzelnen Streamersegmente aber nur von einzelnen Blasen durchlaufen werden.

\section{Vergleich mit einem Aggregationsmodell}

Scheffczyk [94] hat aufgrund der augenscheinlichen Ähnlichkeit mit Streamerstrukturen deterministische Aggregationsmodelle mit verschiedenen Kraftgesetzen zwischen den einzelnen Partikeln

$$
F_{i}\left(r_{N+1}\right)=\frac{r_{N+1}-r_{i}}{\left|r_{N+1}-r_{i}\right|^{k+1}}
$$




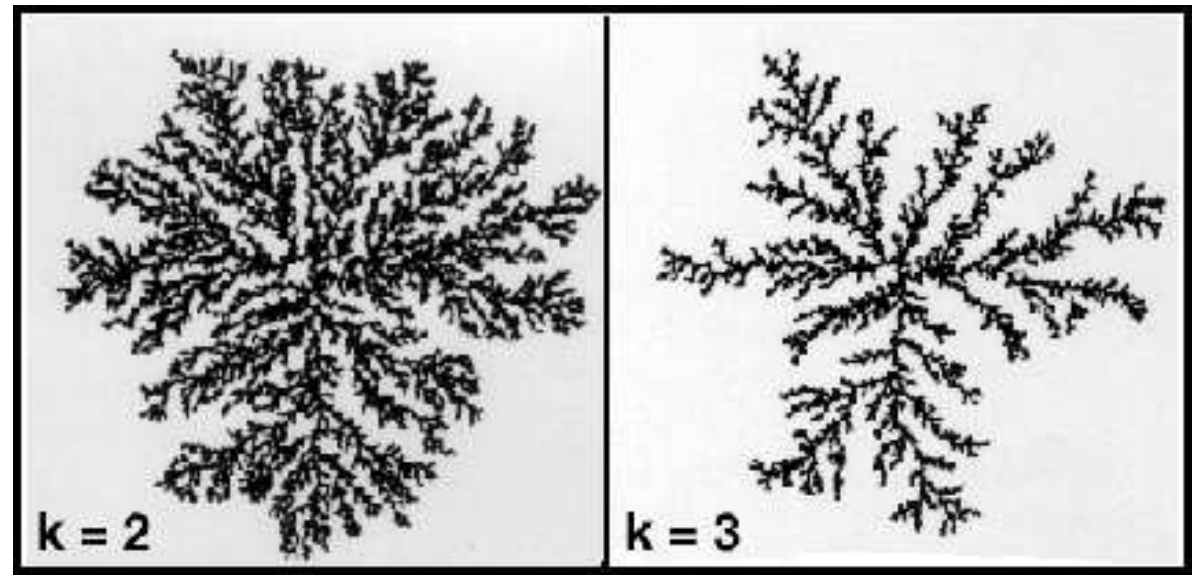

Abbildung 8.16: Deterministische Aggregationsmodelle mit verschiedenen Kraftgesetzen. $\mathrm{k}=2$ entspricht der Ordnung der sekundären Bjerkneskraft. Abbildung aus [94].

gerechnet und die jeweilige fraktale Dimension bestimmt.

Abbildung 8.16 gibt die Ergebnisse der Simulation wieder, deren fraktale Dimension mit einer der ermittelten Dimensionen übereinstimmt. Für den Exponenten $k=2$, der die Entfernungsabhängigkeit der sekundären Bjerkneskraft beschreibt, ergibt sich eine fraktale Dimension von 1.8. Dies entspricht gerade der fraktalen Dimension der Starfish-Strukturen. Die fraktale Dimension der akustischen Lichtenbergfiguren korrespondiert mit dem Exponenten $k=3$. Ein direkter Vergleich zwischen Blasenstrukturen und Aggregationsmodell ist schwierig, da die Aufnahmen Blasentrajektorien wiedergeben, während das Modell aggregierte Partikel beschreibt. Eine gewisse Anwendbarkeit des Modells ist jedoch dadurch gegeben, das sich ein Filament aus vielen auf den gleichen Trajektorien laufenden Blasen zusammensetzt, zu einem Zeitpunkt t also ein Filament mit zahlreichen „Partikeln"besetzt ist. Die Übereinstimmung der Dimension zwischen den StarfishStrukturen und dem $\mathrm{k}=2$ - Modell ist ein Indiz dafür, das in dieser Struktur die zweite Bjerkneskraft dominierend ist. Dort scheint auch eine Gleichverteilung der Blasenquellen in einem festen Abstand zum Zentrum der Struktur gut gegeben zu sein. Die akustische Lichtenbergfigur hingegen ist sehr stark durch die vorhandenen Blasenquellen geprägt, so dass sich per se eine Ungleichverteilung der Startpositionen ergibt, also von dem Modell schlecht beschrieben wird. 


\section{Kapitel 9}

\section{Akustisches Kavitationsspektrum und räumliche Strukturbildung}

1952 misst Esche das akustische Spektrum des Kavitationsgeräusches [28, 79]. Neben der Anregungsfrequenz und einem breiten Rauschuntergrund treten höhere Harmonische und Subharmonische der Anregungsfrequenz auf, die von Lauterborn 1970 durch numerische Simulationen als nichtlineare Resonanzen kleiner Blasen erklärt werden können [60, 62, 82].

\subsection{Modulation der Amplitude mit einem Dreieck- signal}

Bei Kavitationsexperimenten an einem in ein Wasserbecken getauchten, piezoelektrischen Hohlzylinder konnte bei kontinuierlichem Anstieg der Piezospannung Periodenverdopplung im akustischen Spektrum bis zu Periode 8 gemessen werden $[42,15,64]$. Der zeitliche Verlauf der Anregungsamplitude, insbesondere die Steigung des Anstieges, führte dabei zu unterschiedlicher Entwicklung der subharmonischen Anteile. Aber auch bei gleichem Versuchsaufbau konnten die individuellen Messungen sehr unterschiedlich ausfallen und waren schlecht reproduzierbar $[42,45]$. Insbesondere der Zustand des Wassers (frisch, einige Zeit abgestanden, bereits beschallt) hatte einen großen Einfluss auf das Kavitationsgeräusch. Verantwortlich wurden dabei vor allem Unterschiede in der Anzahl und Verteilung der Kavitationskeime gemacht. Diese Beobachtungen deuten darauf hin, dass Strukturbildungsprozesse eine Rolle bei der Entstehung der Subharmonischen spielen können.

Im Plexiglasquader (siehe Anhang A.2) können bei konstanter Anregungsamplitude selbst bei maximaler Anregung keine subharmonischen Schwingungen ge- 
messen werden. Bei linearem Amplitudenanstieg schwingt jedoch oft kurzzeitig im Frequenzspektrum die Linie bei halber Anregungsfrequenz $f=f_{0} / 2$ an, die im folgenden kurz Subharmonische genannt wird. Zeitgleich wird meist das Einsetzen der Linie bei $f=3 f_{0} / 2$ beobachtet, im folgenden als Ultraharmonische bezeichnet. Die simultane Aufzeichnung der Blasenstruktur und des Hydrofonsignals ermöglicht eine Untersuchung des Zusammenhangs zwischen Strukturbildung und dem Anschwingen subharmonischer Frequenzen.

\subsubsection{Durchführung}

Die gesamte Küvette wird mit einer Bildwiederholfrequenz von $100-200 \mathrm{~Hz}$ im Streulicht aufgenommen, gleichzeitig wird das Signal des in einer oberen Ecke der Küvette positionierten Hydrofons von dem 16-bit A/D-Wandler mit einer Abtastrate von $102.4 \mathrm{kHz}$ aufgezeichnet. Die Anregungsamplitude wird mit einem Dreiecksignal moduliert. Die Anstiegszeit von Null bis zur Maximalspannung $\left(U_{F G}=20 \mathrm{Vpp}, U_{p} \approx 1800 \mathrm{Vpp}\right)$ beträgt $t_{a}=0.5-1.5 \mathrm{~s}$. Dieser Modulationszyklus wird bis zum Ende der Aufnahmezeit, die zwischen t $=5-10 \mathrm{~s}$ liegt, wiederholt. Der Bildabstand beträgt je nach Messdauer 5 - 10 ms. Zur Ermittlung des Spektrums wird die Fensterlänge auf den Bildabstand abgestimmt (512 - 1024 Punkte), die Daten werden vor der Fouriertransformation mit einem Hann-Fenster multipliziert.

\subsubsection{Ergebnisse}

In Abbildung 9.1 ist das auf das Maximum der Anregungsfrequenz normierte Amplitudenspektrum des Schallsignals für eine Anstiegszeit von 0.5 s aufgetragen.

Der Doppelpuls markiert den Start der Amplitudenmodulation. Gut reproduzierbar ist das Einsetzen und Abklingen des Breitbandrauschens, das etwa symmetrisch um das Maximum der Anregungsamplitude liegt. Nahe dem Maximum setzt eine Amplitudenmodulation der Anregungsfrequenz und der zweiten Harmonischen mit einer Frequenz um etwa $\mathrm{f}=f_{\text {res }} / 8$ ein, möglicherweise das Anschwingen einer Biegeschwingung der Resonatorwände. Die Amplitude fällt oberhalb der zweiten Harmonischen wegen der Verwendung eines Anti-Aliasingfilters stark ab. Besonders auffällig ist aber das immer stärkere Anschwingen der Subharmonischen und der Ultraharmonischen beim Spannungsanstieg. Bei der ersten Modulation kann die Subharmonische als recht breite schwache Linie erahnt werden, die sich kaum vom Rauschpegel abhebt, beim vierten Amplitudenanstieg ist 


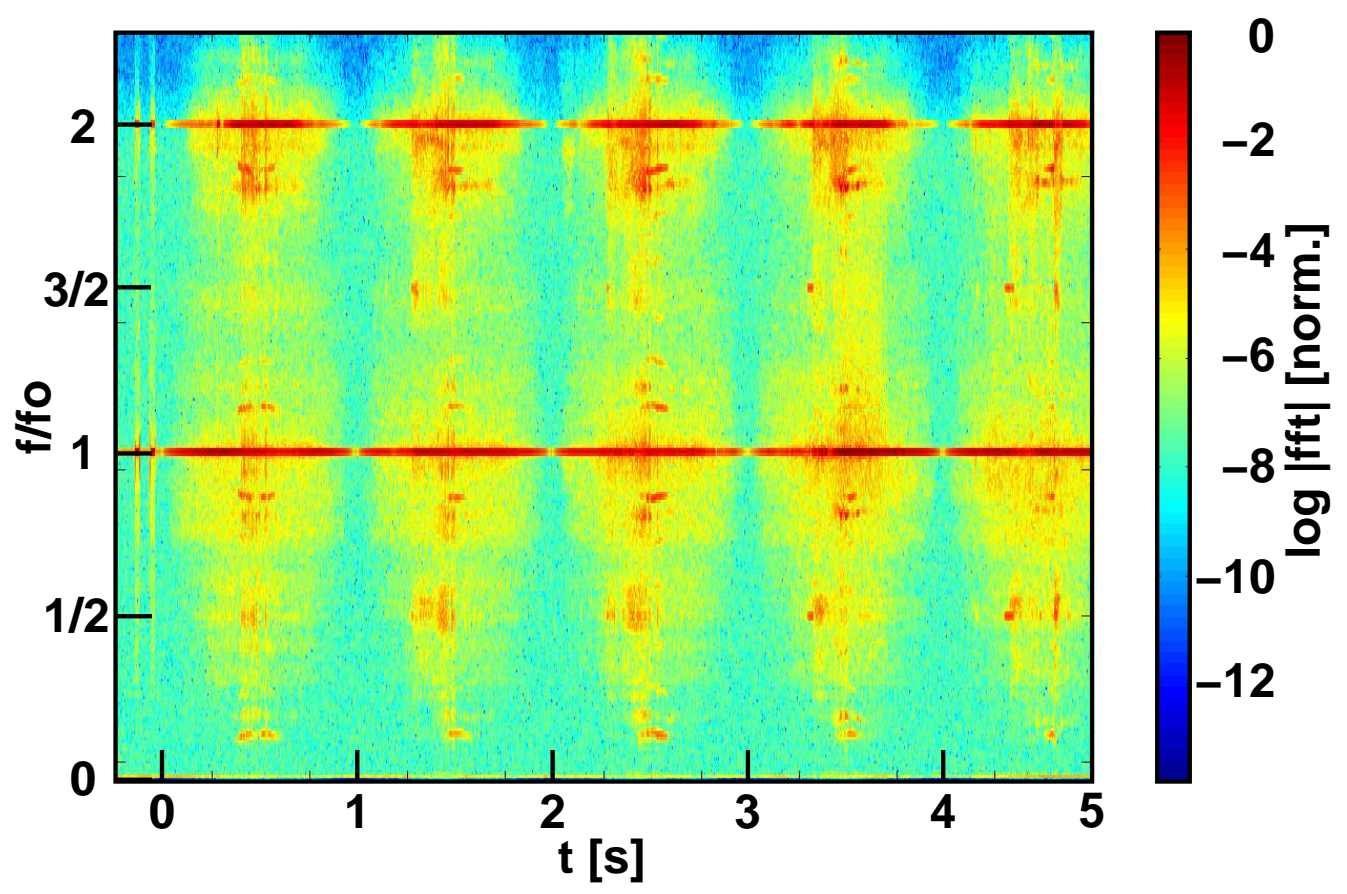

\begin{abstract}
Abbildung 9.1: Spektrogramm des Hydrofonsignals im Plexiglasquader bei wiederholter Modulation der Anregung mit einem Dreiecksignal, Anstiegszeit 0.5 s. Die Amplituden der Spektralkomponenten sind logarithmisch skaliert, normiert ist auf die Maximalamplitude der Anregungsfrequenz von $22.4 \mathrm{kHz}$.
\end{abstract}

die Subharmonische klar zu erkennen und schwingt mehrere Millisekunden an, ebenso wie die Ultraharmonische. In Abbildung 9.2 ist der zeitliche Verlauf der Anregungsfrequenz (a), der Subharmonischen (b), der Ultraharmonischen (c) und der zweiten Harmonischen (d) aufgetragen. 


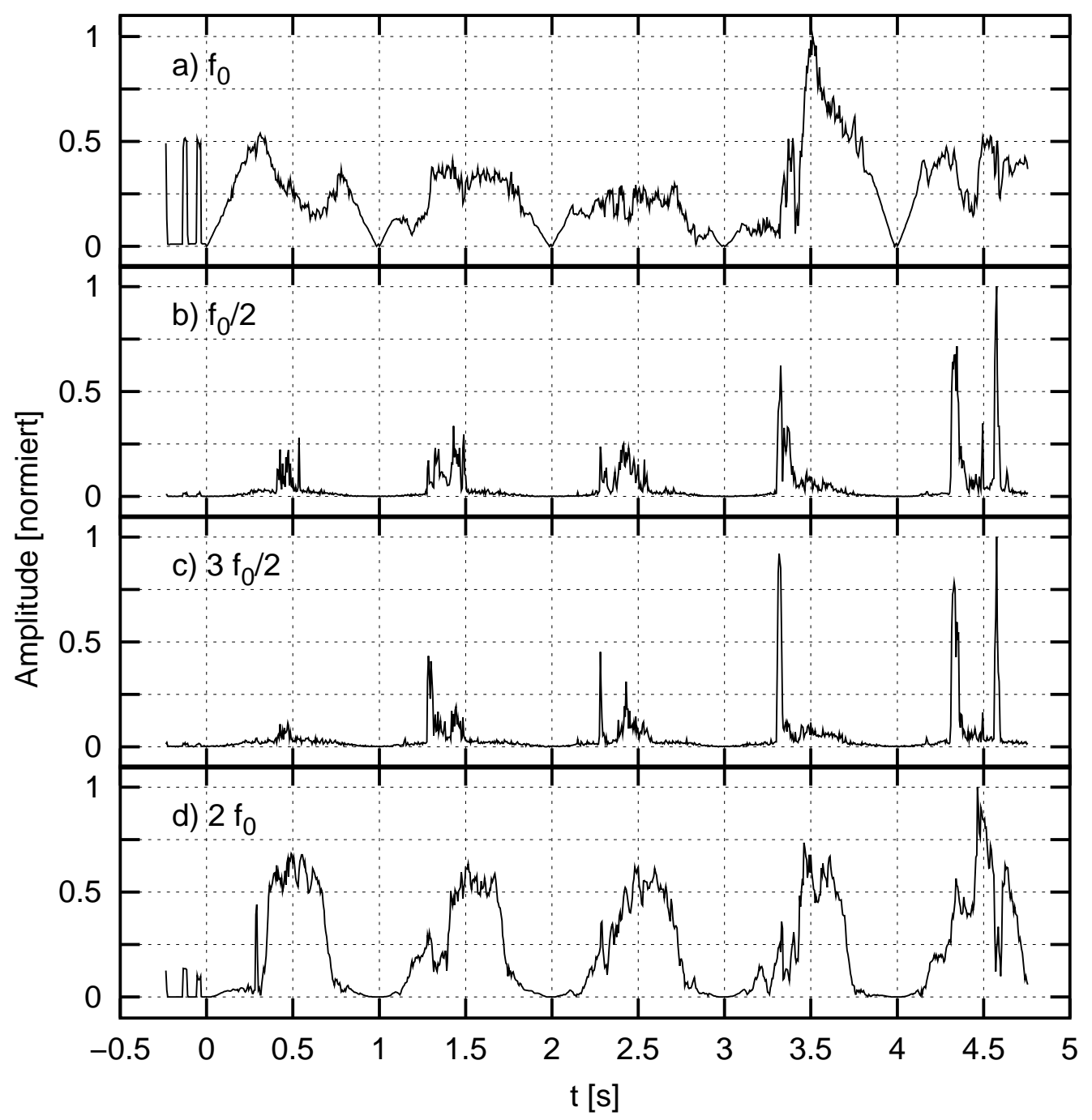

Abbildung 9.2: Zeitlicher Amplitudenverlauf der Anregungsfrequenz $f_{0}$ (a), der Subharmonischen (b), der Ultraharmonischen (c) und der zweiten Harmonischen (d) aus Abbildung 9.1. 
Der Amplitudenverlauf der Anregungsfrequenz in einem Modulationszyklus variiert stark und ist weit entfernt von einem Dreiecksignal. Im dritten Zyklus erreicht die Amplitude lediglich ein Viertel des im darauffolgenden Zyklus erreichten Maximalwertes. Im ersten Zyklus gibt es zwar einen merklichen Anstieg der Subharmonischen, sie hebt sich aber nicht scharf im Spektrum ab (vergleiche Abbildung 9.1). Das Auftreten der Subharmonischen ist nicht symmetrisch um das Maximum der Anregungsamplitude und sie klingt mit Ausnahme des letzten Zyklus bereits kurz nach Erreichen des Maximums ab. Ab dem zweiten Zyklus schwingt die Subharmonische deutlich früher an als im ersten, und bildet ab dem dritten Zyklus einen deutlichen Peak beim Einsetzen, der nach wenigen Millisekunden abfällt, dieser entspricht der scharfen Linie im Spektrogramm. Dieses starke kurze Anschwingen der Subharmonischen und das anschließenden Abfall auf eine niedrigere Amplitude findet sich auch in den oben zitierten Arbeiten. Im letzten Zyklus schwingt die Subharmonische noch einmal im Maximum der Anregung und beim Abklingen an, jedoch ist dort auch das Breitbandrauchen sehr stark, so dass die Linie nicht scharf hervortritt. In Abbildung 9.3 sind zum Vergleich die Kurzzeitspektren bei maximalem Anschwingen der Subharmonischen im letzten Zyklus vor und nach dem Anregungsmaximum dargestellt.

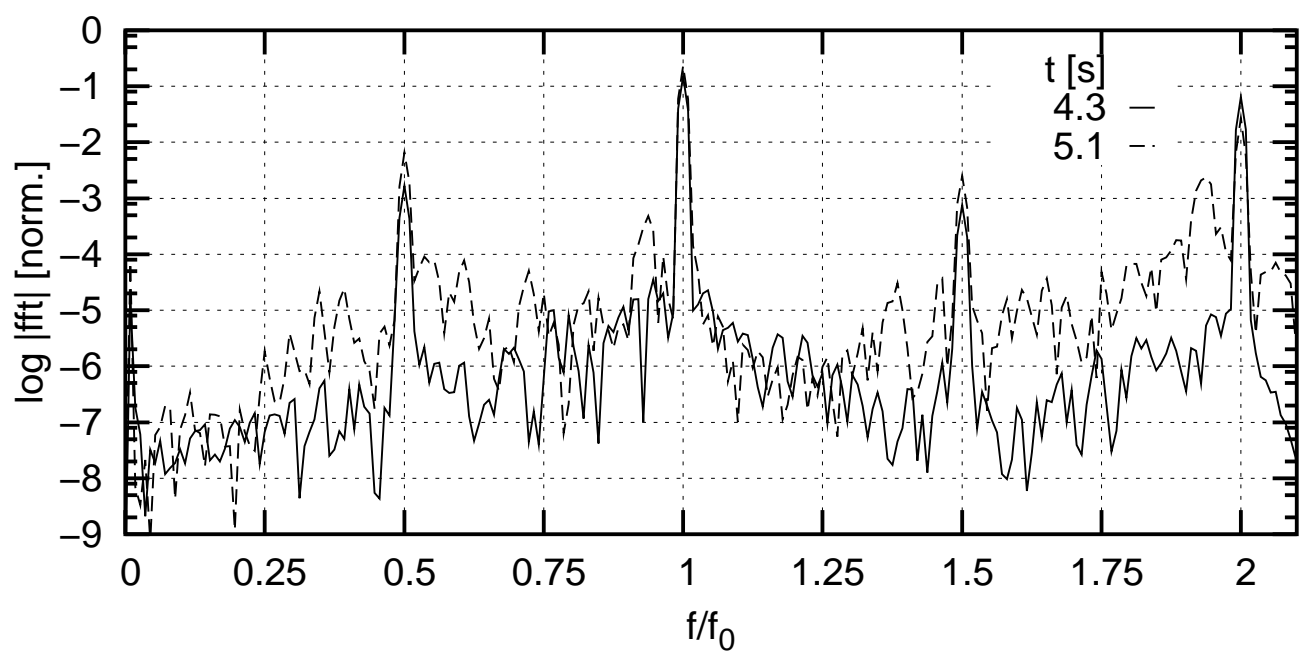

Abbildung 9.3: Akustisches Spektrum bei Anwesenheit der Subharmonischen bei Spannungsanstieg $(\mathrm{t}=4.3 \mathrm{~s})$ und fallender Spannung $(\mathrm{t}=5.1 \mathrm{~s})$. Ausschnitt aus Abbildung 9.1.

Ein Vergleich zwischen dem Bildmaterial und dem akustischen Spektrum zeigt einen Koinzidenz zwischen der Strukturbildung und dem Anschwingen und Ab- 
klingen der Subharmonischen. In Abbildung 9.4 ist für alle fünf Modulationszyklen die Kavitationsstruktur im Zeitbereich des Anschwingens der Subharmonischen abgebildet. $t_{r e l}=0$ entspricht dabei einer Messzeit von $\mathrm{t}=275 \mathrm{~ms}$. Darunter ist der zeitliche Verlauf der Subharmonischen dargestellt. Der besseren Darstellbarkeit der Spektren halber sind dabei die Zyklen von unten nach oben sortiert. Die unterste Bildsequenz (a) entspricht dem ersten Zyklus, die oberste (d) dem fünften.

Im ersten Zyklus (Sequenz (a)) bildet sich keine Streamerfigur aus. Es bilden sich hier Cluster, (zum Beispiel in der linken oberen Ecke), für die der Schalldruckbauch bereits repulsiv ist. Im zweiten und dritten Zyklus ((b) und (c)) sind Streamerstrukturen anfangs sichtbar, die Subharmonische schwingt kurz an, klingt aber bei der Zerstörung der Struktur wieder ab. Beim zweiten Modulationsdurchlauf (b) schwingt sie noch einmal ohne sichtbaren Zusammenhang mit der Kavitationsstruktur für etwa $40 \mathrm{~ms}$ an $\left(t_{r e l}=40 \mathrm{~ms}\right)$. Ein Vergleich mit dem Spektrum zeigt auch hier den Unterschied zwischen der scharfen Linie beim ersten Anschwingen und einem relativ breit gestreuten Spektrum im Bereich der zweiten Amplitudenzunahme bei $t_{r e l}=40 \mathrm{~ms}$. Im weiteren Verlauf der Messung ist eine deutliche Zunahme der an der Struktur beteiligten Blasen und die Verschiebung des Zerstörungspunktes der Streamerfigur nach hinten zu beobachten. Ebenso verschiebt sich der Zeitpunkt des Einsatzes der Subharmonischen. Eine starke Veränderung der Struktur ist dabei nicht auffällig. Das plötzliche Abklingen der Subharmonischen $\left(t_{r e l}=60 \mathrm{~ms}\right.$ bei $(\mathrm{d}), t_{r e l}=85 \mathrm{~ms}$ bei $\left.(\mathrm{e})\right)$ jedoch findet zeitgleich mit der Zerstörung der Struktur und deren Aufösung in einzelne Cluster statt. Nach der Zerstörung ist wie in Zyklus (b) die Subharmonische noch messbar, aber verrauscht. Der gleiche Effekt der „Konditionierung des Kavitationsfeldes"ist auch in anderen Messungen zu beobachten. In Abbildung 9.5 sind die analogen Messungen für eine Anstiegszeit von $t_{a}=1 \mathrm{~s} \mathrm{(a)} \mathrm{und} t_{a}=1.5 \mathrm{~s}$ (b) dargestellt. Normierung und Skalierung sind wie in Abbildung 9.1.

Bei langsamer Anstiegszeit (Abbildung 9.5 (b)) nimmt die Stärke der Subharmonischen über die drei Modulationszyklen zu, während bei $t_{a}=1 \mathrm{~s}$ (Bild (a)) die Amplitude zwar auch vom ersten zur zweiten Modulationszyklus zunimmt, dann aber abwechselnd schwächer und stärker wird. In Abbildung 9.6 ist für diese Messreihe der Zeitverlauf der Subharmonischen und die entsprechenden Bildsequenzen analog zu Abbildung 9.4 abgebildet.

Auch hier gilt, je später die Subharmonische einsetzt, eine desto höhere Amplitude erreicht sie. Und auch hier bricht die Subharmonische zeitgleich mit der Struktur zusammen. Die sehr gut reproduzierbare Lage der Streamer spricht im Übrigen dafür, dass die Orte der Blasenquellen nahezu konstant sind. 


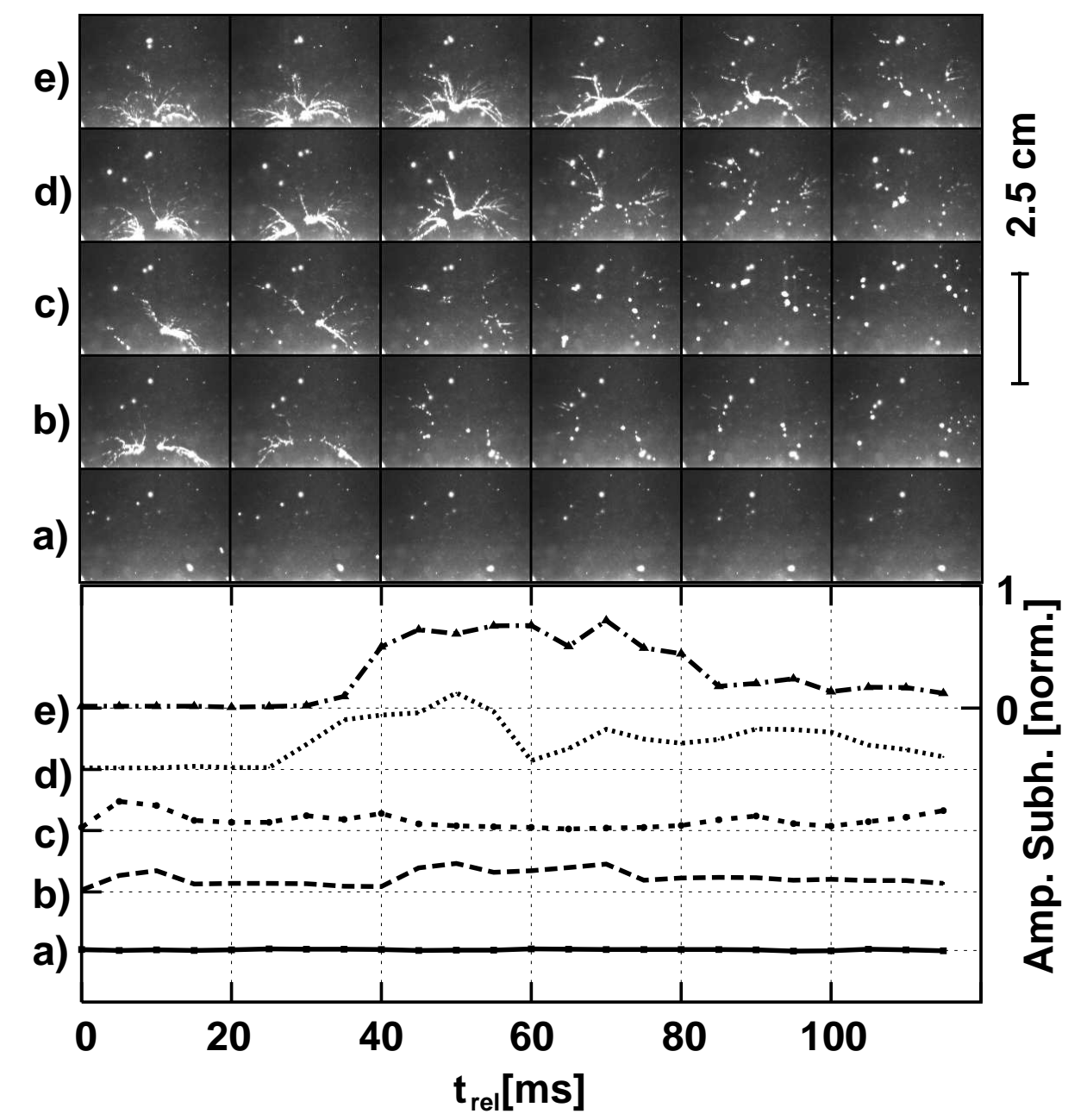

Abbildung 9.4: Vergleich zwischen Kavitationsstruktur und dem Verlauf der Subharmonischen bei linearer Spannungsänderung (siehe Abbildung 9.1). Die Bildzeilen geben Sequenzen aus den Einzelmessungen wieder. Dabei entspricht Sequenz (a) dem ersten Modulationszyklus, Sequenz (e) dem fünften. Die Sequenzen beginnen 275 ms nach Beginn des Spannungsanstieges, also bei $55 \%$ der Maximalspannung $\left(U_{F G}=11 \mathrm{Vpp}\right)$, der Bildabstand beträgt $20 \mathrm{~ms}$. Unter den Bildsequenzen ist der Verlauf der auf den Maximalwert normierten Amplitude der Subharmonischen abgebildet. Dem Bild entspricht der jeweils erste Datenpunkt im durch die Bildkanten gegebenen Intervall. 

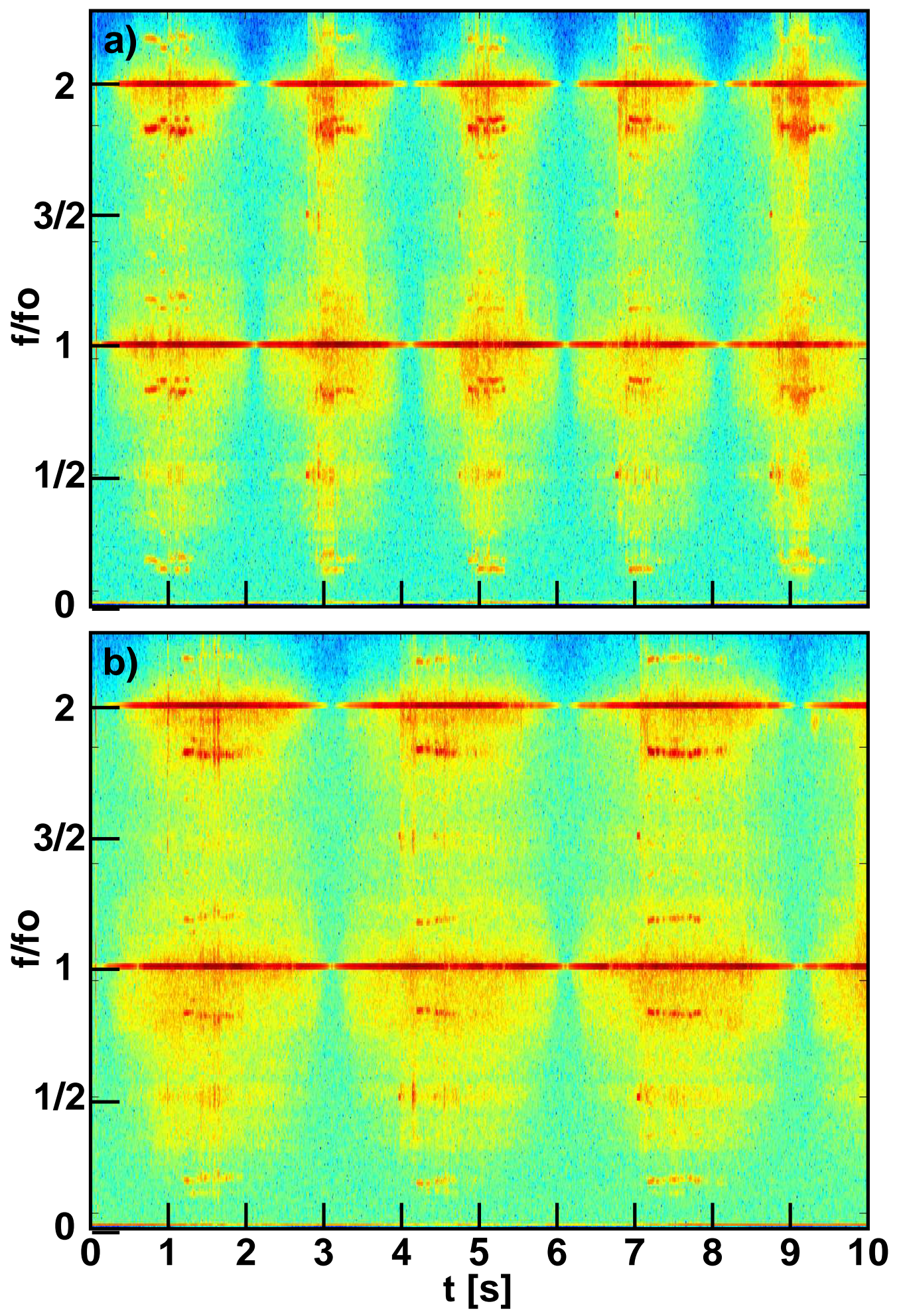

Abbildung 9.5: Spektrogramm des Hydrofonsignals bei wiederholter Modulation der Anregung mit einem Dreiecksignal, Anstiegszeit $t_{a}=1 \mathrm{~s} \mathrm{(a)}$ und $t_{a}=1.5 \mathrm{~s}$ (b). Skalierung und Normierung wie in Abbildung 9.5 . 


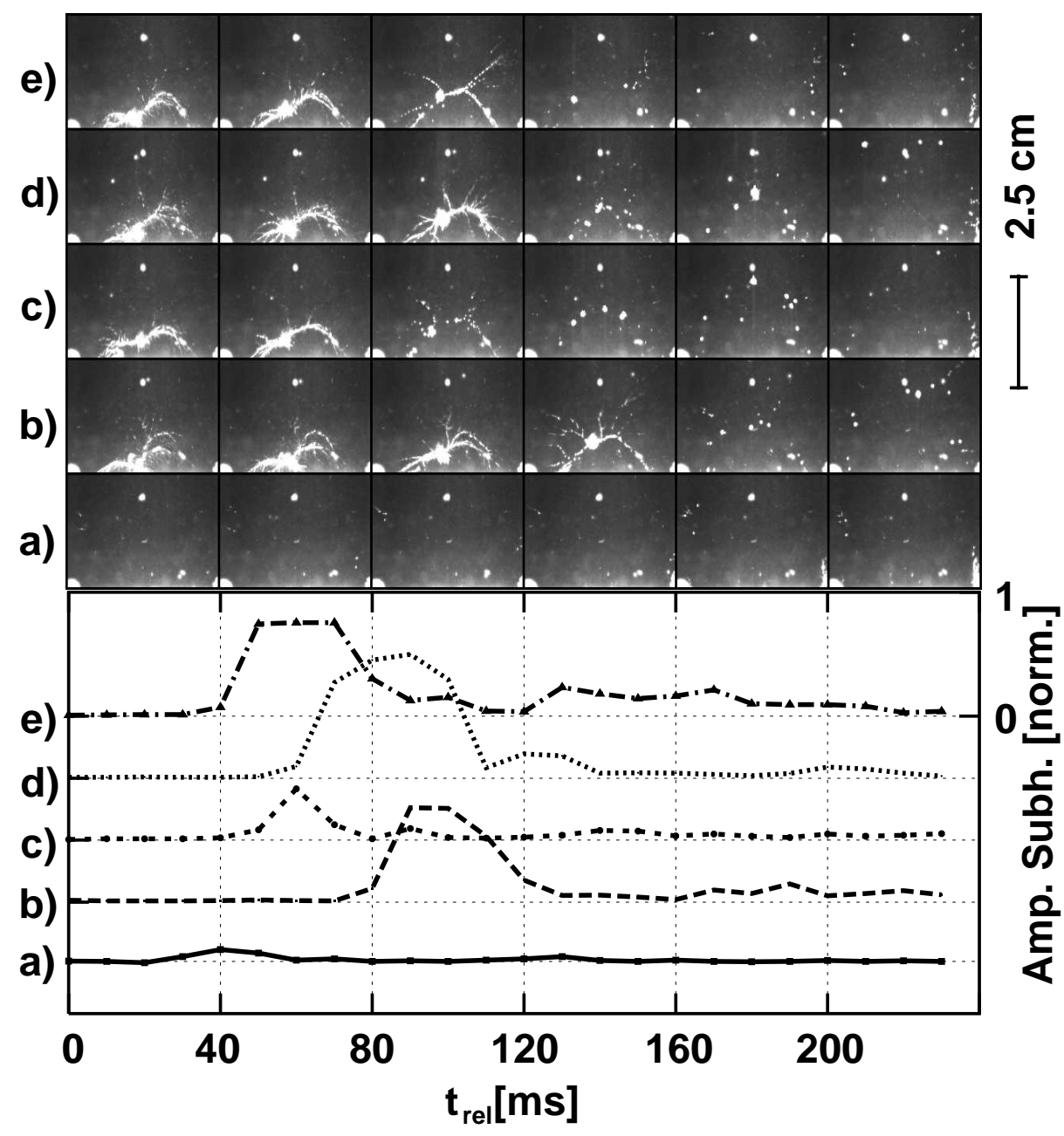

Abbildung 9.6: Vergleich zwischen Kavitationsstruktur und dem Verlauf der Subharmonischen bei linearer Spannungsänderung, $t_{a}=1 \mathrm{~s}$ (siehe Abbildung 9.5 (a)). Die Sequenzen beginnen 560 ms nach Beginn des Spannungsanstieges, also bei $56 \%$ der Maximalspannung $\left(U_{F G}=11.5 \mathrm{Vpp}\right)$, der Bildabstand beträgt $40 \mathrm{~ms}$. Zur weiteren Erläuterung der Grafik siehe Abbildung 9.4. 
Der exakte Moment des Einsetzens der Subharmonischen scheint, wenn die Blasenquellen erst einmal durch vorherige Beschallung installiert sind, von statistischen Größen abzuhängen.

Die gleiche Versuchsdurchführung wird in einer größeren Plexiglaswanne wiederholt (siehe Anhang A.3), die nicht in Resonanz des Wasservolumens, sondern in Resonanz der am Boden befestigten Schallwandler betrieben wird. Das Hydrofon ist wieder nur etwa $1 \mathrm{~cm}$ in das Wasser eingetaucht, positioniert an eine Knotenstelle der Schwerewelle. Der Spannungsanstieg beträgt diesmal $2.5 \mathrm{~s}$. In Abbildung 9.7 ist das Amplitudenspektrum analog zu Abbildung 9.1 dargestellt.

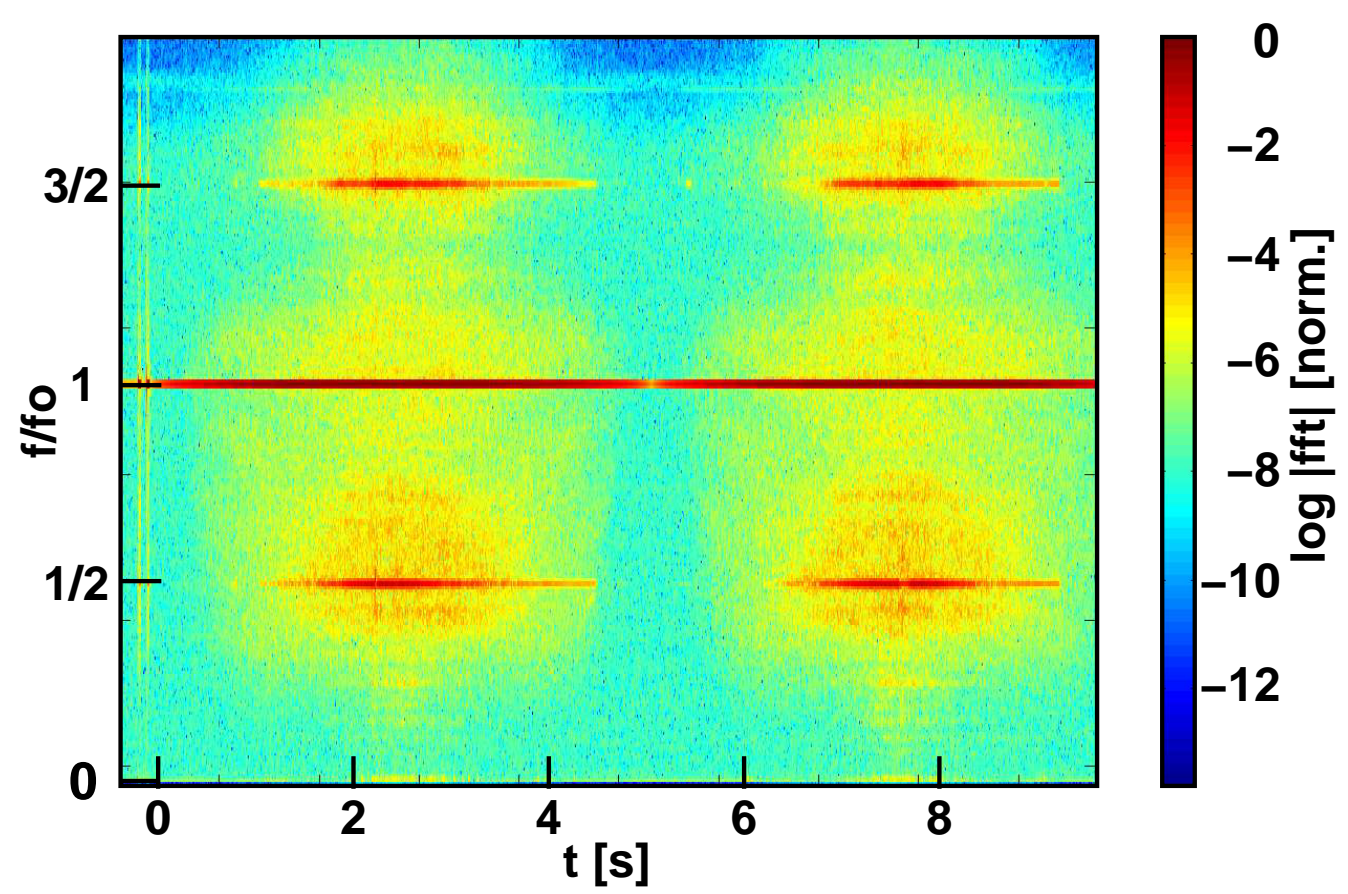

Abbildung 9.7: Spektrogramm des Hydrofonsignals bei zweimaliger Amplitudenmodulation mit einem Dreiecksignal in der Plexiglaswanne. Anstiegszeit $t_{a}=2.5 \mathrm{~s}$. Skalierung und Normierung wie in Abbildung 9.1. 


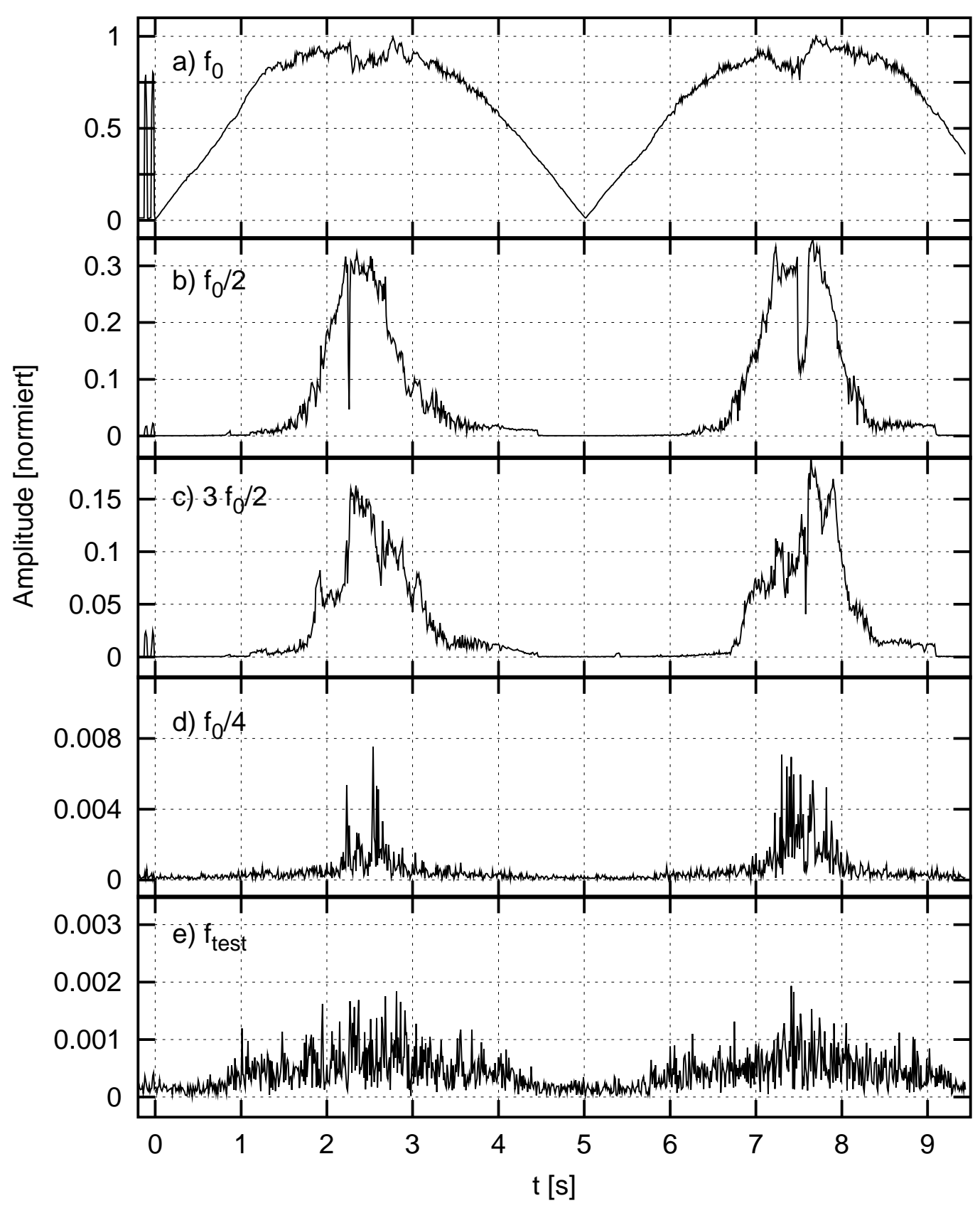

Abbildung 9.8: Zeitlicher Amplitudenverlauf der Anregungsfrequenz $f_{0}$ (a), der Subharmonischen $f_{0} / 2$ (b), der Ultraharmonischen $3 f_{0} / 2$ (c), der subharmonischen Periode 4 Schwingung $f_{0} / 4$ (d) und einer Frequenz $f_{\text {test }}=f_{0} / 4+1 \mathrm{kHz}$ (e) ohne einfaches rationales Verhältnis zur Anregungsfrequenz aus Abbildung 9.7. 
Bei dieser Messung ergibt sich ein anderes Bild. Die Subharmonische setzt erst im Rauschen ein und erhöht sich dann kontinuierlich. Zeitgleich mit der Subharmonischen setzt die Ultraharmonische ein. Auch ein schwacher Anteil der Periode 4 Schwingung ist in der Nähe des Anregungsmaximums auszumachen. Bei fallender Amplitude nimmt die Subharmonische zuerst nahezu symmetrisch um das Maximum ab, tritt dann jedoch bei nahezu konstanter Amplitude immer stärker aus dem abnehmenden Breitbandrauschen hervor, um dann abrupt abzubrechen. Dieses Verhalten ist in beiden Modulationszyklen zu beobachten. In Abbildung 9.8 ist der zeitliche Amplitudenverlauf der Grundmode $f_{0}$ (a), der Subharmonischen (b), der Ultraharmonischen (c) und der subharmonischen Periode 4 Schwingung $f=f_{0} / 4(\mathrm{~d})$ abgebildet. Zusätzlich ist der Verlauf einer Testfrequenz $f_{\text {test }}=f_{0} / 4+1 \mathrm{kHz}$ dargestellt (e), um einen Eindruck von der Entwicklung des Breitbandrauschens zu vermitteln.

Der Vergleich mit dem Amplitudenverlauf der Testfrequenz zeigt, dass sich die Periode 4 Schwingung kurzzeitig aus dem Rauschpegel ihrer spektralen Umgebung hervorhebt, also tatsächlich signifikant anschwingt. Sowohl die Subharmonische als auch die Ultraharmonische zeigen eine Hystereseeffekt auf dem abklingenden Ast des Dreiecksignals. Interessant ist, dass die Ultraharmonische in beiden Modulationszyklen nach einem der Subharmonischen äquivalenten Amplitudenverlauf in etwa konstant bleibt, und erst nach dem kurzzeitigen Einbruch der Subharmonischen wieder die gleiche Amplitude erreicht. Aufgrund eines Triggerfehlers können die Bilder nur mit einer Genauigkeit von 30 ms der Zeitreihe zugeordnet werden, so dass Aussagen auf kurzer Zeitskala nur bedingt getroffen werden können. Die Einbrüche in der Amplitude der Subharmonischen lassen sich mit spontan entstehenden Clustern in Verbindung bringen. In Abbildung 9.9 ist die Clusterentstehung im ersten Modulationszyklus wiedergegeben. Die Bildsequenz zeigt zu Beginn zwei Kavitationsstrukturen am Boden der Wanne, die auf den Befestigungsschrauben des vorderen Schallwandlers lokalisiert sind.

In der Mitte des Bildes befinden sich Cluster im Übergang zu Jellyfish-Strukturen. Im zweiten Bild ist der spontan entstandene Cluster zu sehen, der sich anschließend mit den bereits im Volumen befindlichen Clustern vereinigt. In der Grafik ist der Zeitverlauf der Subharmonischen (durchgezogene Linie und Punkte), der Anregungsfrequenz (gestrichelte Linie) und der Ultraharmonischen (gepunktete Linie) wiedergegeben. Um die Variabilität der Linien einordnen zu können, wurde der Verlauf für einen etwa dreimal so langen Zeitraum wiedergegeben. Die Amplituden sind hier auf das Maximum der jeweiligen Linie normiert. Die schwarzen Punkte geben den Fehlerbereich für das erste Bild in der Sequenz an. Es ist zwar evident, dass der Cluster die Ursache für den Einbruch der Subharmoni- 


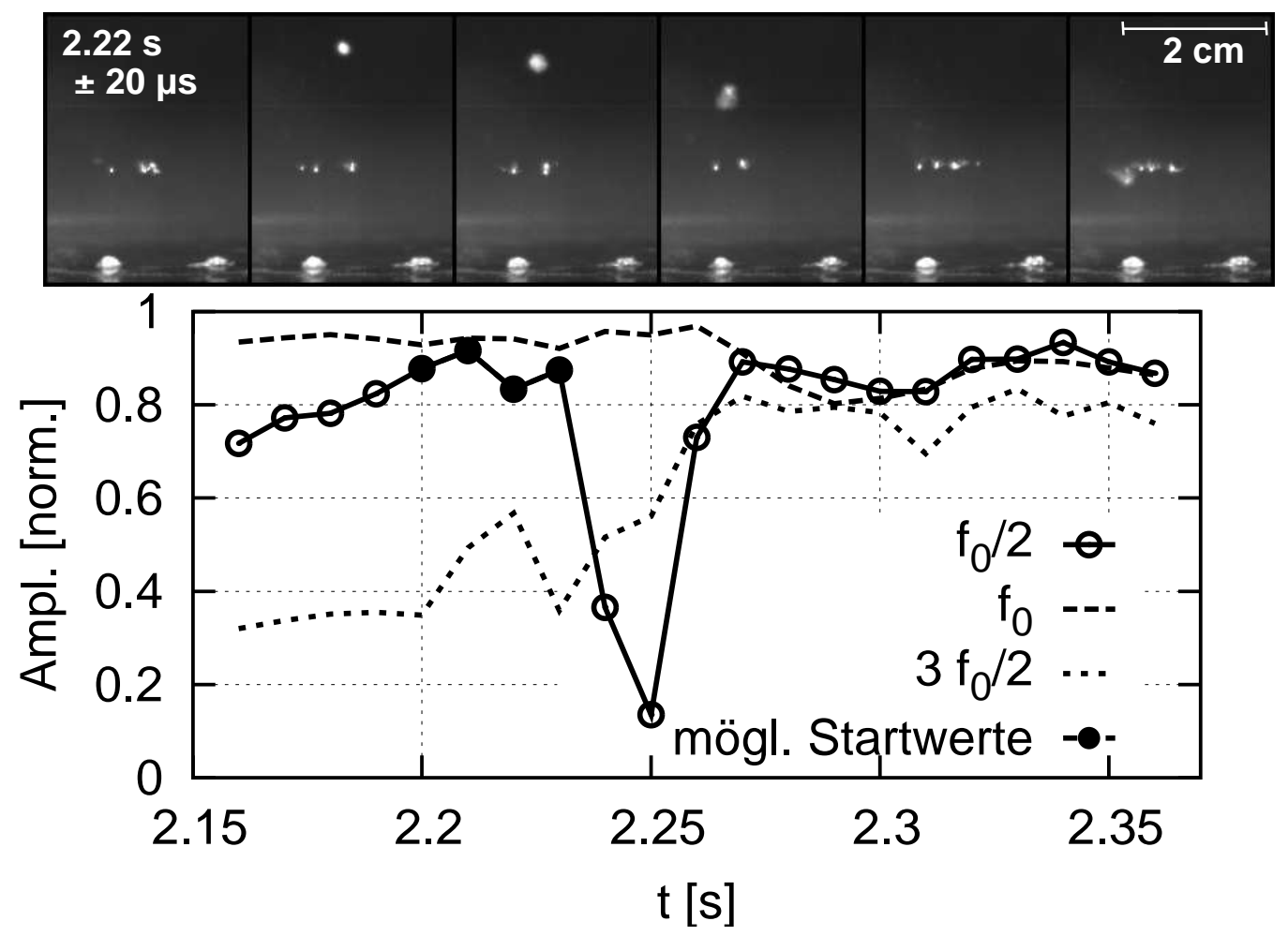

\begin{abstract}
Abbildung 9.9: Spontane Clusterentstehung und der Verlauf der Subharmonischen. Der Cluster verursacht einen starken Abfall der Amplitude der Subharmonischen (gestrichelte Linie), aus mangelnder zeitlichen Auflösung ist jedoch nicht ersichtlich, ob der Abfall durch die Entstehung des Clusters oder aufgrund der Kollision mit den bereits existierenden Strukturen zustande kommt.
\end{abstract}

schen ist, jedoch kann anhand der Datenlage nicht geklärt werden, ob bereits die Entstehung des Clusters die Subharmonische beeinträchtigt, oder erst die Kollision mit den bereits bestehenden Strukturen. Die Ursache für den anschließenden Anstieg der Ultraharmonischen ist unklar, da sich die sichtbaren Strukturen in ihrem Erscheinungsbild nicht signifikant ändern. Im zweiten Modulationszyklus, in dem der gleiche Effekt auftritt, vereinigt sich der transiente Cluster nicht mit den bestehenden Strukturen, sondern wird zur Seite gezogen, bildet dort eine Jellyfish-ähnliche Struktur, die nach wenigen Millisekunden in einzelne Cluster zerfällt, etwa zeitgleich mit dem erneuten Anschwingen der Subharmonischen.

Unabhängig von der zeitlichen Auflösung ist die Zuordnung einer bestimmten Struktur zu dem Entstehen oder Abfallen der Subharmonischen schwierig, da diese ja nicht per se subharmonisch oder nicht subharmonisch schwingt, sondern durch die Wechselwirkung innerhalb der Struktur die Möglichkeit einer kollekti- 
ven Periodenverdopplung bietet. Eher ist das Verschwinden der Subharmonischen bei Zerstörung einer Struktur ein Hinweis darauf, dass die Struktur sich vorher in der Periodenverdopplung befunden hat. So sind bei dem langsamen Einsetzen der Subharmonischen bei Amplitudenerhöhung sowohl Kavitationsaktivitäten an den Schrauben als auch Cluster im Wasservolumen zu beobachten. Der steile Anstieg in der Amplitude geschieht in etwa zeitgleich mit dem starken Aufschwingen der hemisphärischen Strukturen an den Schrauben (siehe Abbildung 9.9), aber auch die aus den Clustern entstehenden Jellyfish-Strukturen werden größer und wirken blasenreicher.

Die Abnahme des subharmonischen Anteils am Kavitationsspektrum geht einher mit der Abnahme der Jellyfish- bzw. Clusterstrukturen im Volumen und dem langsamen Zusammenfallen der Kavitationsstrukturen an den Schrauben. Bei dem plötzlichen Verschwinden der Subharmonischen sind im ersten Modulationszyklus bereits seit $100 \mathrm{~ms}$ keine Kavitationsstrukturen mehr im Volumen zu beobachten, von daher liegt die Vermutung nahe, dass die Strukturen um die Schrauben einen beträchtlichen Anteil an der Erzeugung subharmonischer Schallwellen haben. Kurz nach Verschwinden der Subharmonischen beginnen sich Streamer von den Schrauben ins Volumen zu bilden, die erst bei Erreichen des Schalldruckminimums abreißen. Dort befinden sich also auch nach Verschwinden der hemisphärischen Strukturen noch Blasenquellen.

Diese Messungen geben einen Einblick in den Zusammenhang zwischen Kavitationsstrukturen und akustischem Spektrum. Die Messergebnisse sind konsistent mit der Annahme, dass es sich bei der Anregung der Subharmonischen um den Einsatz einer Periodenverdopplungskaskade handelt. Aufgrund der Transformation der Streamerfigur in einzelne Blasencluster kann diese im Plexiglasquader nicht weiter verfolgt werden (siehe Abbildungen 9.4 und 9.6). Hier ist die Verteilung und die Anzahl der Blasenquellen von großer Bedeutung für die Stabilität der Struktur, die bei hoher Blasenanzahl auch bei höherer Anregungsamplitude noch erhalten bleibt. Die scharfe Linie der Subharmonischen im Zusammenhang mit der Streamerstruktur erklärt sich aus dem kollektiven Prozess innerhalb der Struktur, an dem nahezu alle Blasen innerhalb des Resonators beteiligt sind. Das stärker verrauschte Anschwingen der Subharmonischen zu anderen Zeitpunkten der Messung wird möglicherweise von kleineren untereinander nicht korrelierten Blasenstrukturen erzeugt, die aus den Bilddaten schwer zu orten sind. In diesen Zusammenhang ist auch das akustische Spektrum in der Ultraschallwanne einzuordnen (Abbildung 9.7). Die Ergebnisse decken sich im Prinzip mit den früheren Experimenten zum Kavitationsgeräusch, die zu Beginn dieses Abschnitts beschrieben wurden. Insbesondere die hohe Variabilität der Einzelmessungen und 
der Einfluss von vorheriger Beschallung können ebenfalls beobachtet und mit Strukturbildungsprozessen in Verbindung gebracht werden. Die Tatsache, dass im Plexiglasquader die Subharmonische nur transient auftritt, während im zitierten Versuchsaufbau die stationäre subharmonische Schwingung der Kavitationsstruktur holografisch aufgezeichnet werden konnte [65], kann verschiedene Gründe haben. Zum einen ist der dort verwendete piezokeramische Hohlzylinder durch seine Oberfläche vermutlich sehr blasenquellenreich. Zum anderen besitzt die den Zylinder umgebende Wasserwanne akustische Resonanzen im Frequenzbereich unterhalb der Anregungsfrequenz des Zylinders von $23 \mathrm{kHz}$, so dass anschwingende Subharmonische durch Wechselwirkung mit der Wanne stabilisiert werden können, dies allerdings bei der angegebenen Wannengröße bis maximal Periode 4. Ähnliches gilt auch für die Ultraschallwanne, in der ebenfalls die Subharmonische ab einem Schwellwert der Anregungsamplitude dauerhaft anschwingt.

Auch die prinzipiell unterschiedliche Erzeugung der hohen Schalldruckamplituden ist möglicherweise von Bedeutung. Der Hohlzylinder schwingt selber in Resonanz, ebenso werden auch in kommerziell erhältlichen Reinigungswannen die Schallwandler in Resonanz betrieben. Eine Abstimmung zwischen Eigenschwingung des angekoppelten Wasservolumens und der Anregungsfrequenz ist nicht erforderlich, ein Stehwellenfeld bildet sich auch bei schlechter Anpassung durch Einfachreflexion an der Wasseroberfläche oder bei entsprechender Richtcharakteristik der Wandler an den Wänden der Ultraschallwanne. In dem Plexiglasquader wird die Piezokeramik unterhalb ihrer Resonanzfrequenz betrieben, die bei etwa $170 \mathrm{kHz}$ liegt. Die benötigten Schalldruckamplituden werden durch Eigenschwingungen der wassergefüllten Küvette erzeugt. Die Schwingungseigenschaften der Küvette sind aber abhängig vom Gesamtvolumen und der Schallgeschwindigkeit der Flüssigkeit, die durch Kavitation stark verändert werden können (siehe Abschnitt 5.1). Das Schallfeld wird möglicherweise zu stark von den Blasen beeinflusst, so dass eine stationäre Schwingung höherer Periode nicht zustandekommt, während in der Wanne hauptsächlich Blasen im Nahfeld der Wandler Periodenverdopplung vollführen.

\subsection{Reorganisation des Schallfeldes nach dem Umrühren}

Die hörbaren Frequenzen des Kavitationsspektrums verschwinden zusammen mit den Strukturen, wenn die Flüssigkeit durchgerührt wird. Die sich bildenden Blasenschleier formieren sich nach Beendigung der Störung über Clusterbildung zu 
Jellyfish-Strukturen. Dabei schwingt auch die Subharmonische und die Ultraharmonische wieder an. In Abbildung 9.10 ist das Spektrogramm sowie ein Überblick über die Entwicklung der Blasenstrukturen im Abstand von $1 \mathrm{~s}$ gegeben. Auch hier ist eine genaue Zuordnung von Struktur und der Evolution der Subharmonischen aufgrund der Vielfalt der Strukturen und mangelnder Zeitauflösung nicht möglich.

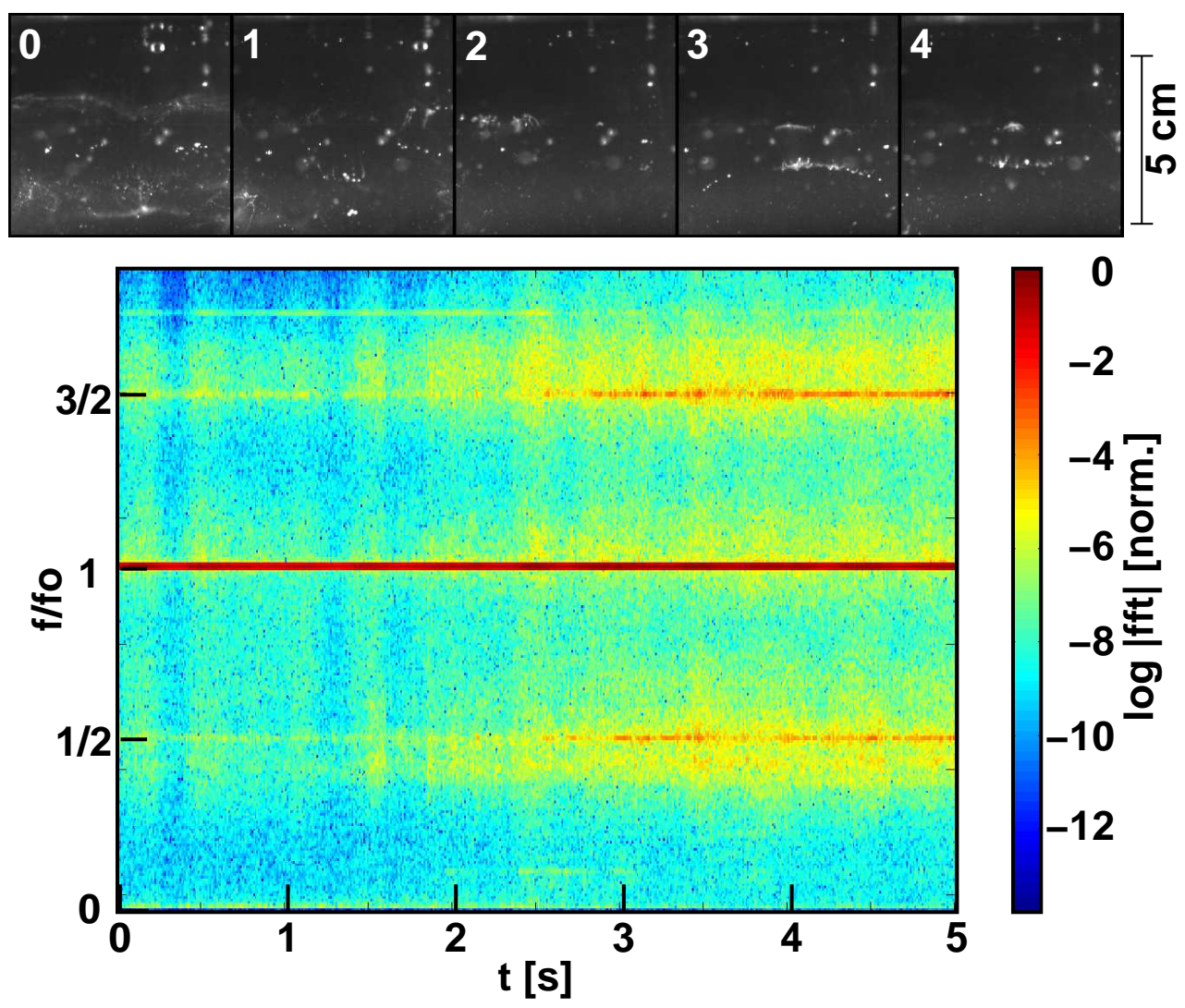

Abbildung 9.10: Reorganisation des Kavitationsfeldes nach dem Umrühren. Die Bildsequenz gibt die Entwicklung der Blasenstrukturen im Abstand von $1 \mathrm{~s}$ wieder, die Farbgrafik das dazugehörige Spektrogramm. 


\subsection{Blasenstruktur an einer Befestigungsschraube}

Bei den Experimenten mit der Ultraschallwanne fällt auf, dass gelegentlich bereits bei vergleichsweise niedriger Anregungsamplitude die Subharmonische deutlich zu hören ist, obwohl im Volumen noch keine Kavitation stattfindet. Als Verursacherin kann eine Blasenstruktur an einer der Befestigungsschrauben ausgemacht werden. Reproduzierbar kann die Subharmonische durch Zerstörung bzw. Induzierung der Struktur manipuliert werden. In Abbildung 9.11 ist der zeitliche Verlauf der Anregungsfrequenz, der Subharmonischen und der Ultraharmonischen wiedergegeben, sowie Bilder der Blasenstruktur im Abstand von 1 s. Die Struktur wird durch ein an einem Metallstab befestigtes Stück Moosgummi gestört, das auf die Schraube gedrückt wird. Das Moosgummi berührt die Schraube zweimal, der Zeitpunkt entspricht dem Amplitudenabfall der Anregungsfrequenz auf 0.5 $(\mathrm{t}=1.1 \mathrm{~s}$ und $\mathrm{t}=1.5 \mathrm{~s})$. Bei $\mathrm{t}=1.7 \mathrm{~s}$ hat der Stab die Wanne bereits wieder verlassen. Dennoch dauert es noch fast zwei Sekunden, bis der Schalldruck der Anregungsfrequenz wieder den Wert vor der Störung erreicht. Vermutlich liegt dies an den verursachten Strömungen in der Wanne (siehe vorigen Abschnitt). Auch die Subharmonische schwingt erst etwa 1.5 s nach Beendigung der Störung wieder an $(\mathrm{t}=3.3 \mathrm{~s})$. Interessanterweise bleibt die Ultraharmonische länger beeinträchtigt. Sie erreicht erst $4.5 \mathrm{~s}$ nach Beendigung der Störung wieder das ursprüngliche Niveau. Äußerlich nimmt die Struktur bereits wenige Millisekunden nach der Störung wieder ihre hemisphärische Form um den Schraubenkopf ein und zeigt auch im weiteren keine auffälligen Veränderungen mehr. Im restlichen Becken entstehen an zwei Stellen gelegentlich transiente Cluster oder Einzelblasen, diese Ereignisse beginnen ebenfalls bereits kurz nach Beendigung der Störung. 

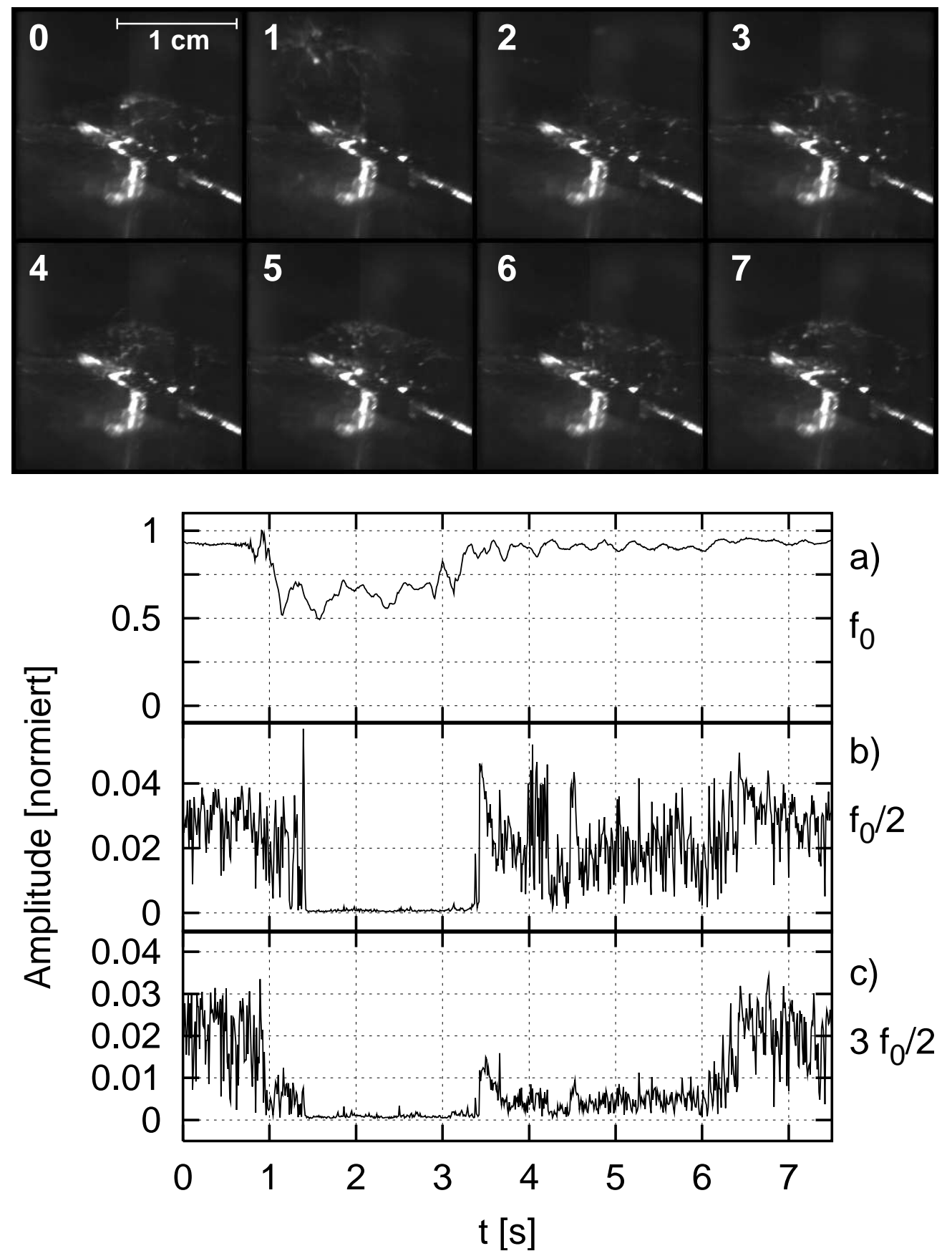

Abbildung 9.11: Störung der hemisphärischen Struktur um eine Befestigungsschraube in der Ultraschallwanne. Aufnahmen der Struktur im Abstand von 1 s (oben) und der Amplitudenverlauf der Anregungsfrequenz $f_{0}$ (a), der Subharmonischen (b) und der Ultraharmonischen (c), normiert auf das Maximum der Anregungsfrequenz. 


\section{Kapitel 10}

\section{Ultraschallreinigung und Kavitationserosion}

Es werden im Ultraschallreaktor (siehe A.4) Reinigungs- und Erosionsversuche durchgeführt, um den Zusammenhang zwischen Kavitationsstrukturen und abgereinigten bzw. erodierten Stellen zu untersuchen [81, 93].

\subsection{Reinigung von Glasplatten}

Als erstes stellt sich die Frage, inwieweit die Orte sichtbarer Blasen mit Orten hoher Reinigungswirkung zusammenfallen. Zum einen müssen nicht zwangsläufig große Blasen am Reinigungsprozess beteiligt sein, zum anderen beeinflussen die Blasenstrukturen das gesamte Schallfeld, so dass auch durch Kavitation induzierte Kräfte an anderer Stelle wirken können. Dazu werden handelsübliche Objektträger mit wasserfestem Faserstift (edding 3000) einseitig bemalt und in das Zentrum des Reaktors gehängt. Durch eine kombinierte Durchlicht- Streulichtillumination erscheinen abgereinigte Stellen grau und Blasenstrukturen weiß im Bild. Nach einer Weile bilden sich horizontale abgereinigte Streifen, die mit den Doppellagen der Jellyfish-Strukturen zusammenfallen. In Abbildung 10.1 ist ein typisches Reinigungsergebnis zu sehen. Die horizontalen Streifen sind gut erkennbar. Im oberen Drittel ist eine Jellyfish-Struktur auf dem Objektträger zu sehen. An den Orten erkennbarer Blasen ist die Farbe abgereinigt. Knapp unter der Mitte des Bildes befindet sich eine weitere Knotenebene, eine sehr schwache JellyfishStruktur ist auf der Platte zu sehen, weiter rechts im Wasservolumen befindet sich eine Struktur auf gleicher Ebene. Im Bereich hohen Schalldrucks (zwischen oberer und unterer Doppelreihe) befinden sich ebenfalls Reinigungsspuren, insbesondere auf der linken Seite. Der Bereich zwischen den Doppellagen, wo sich die Knotenflächen des Schallfeldes befinden, ist keine Abreinigung zu beobachten. 


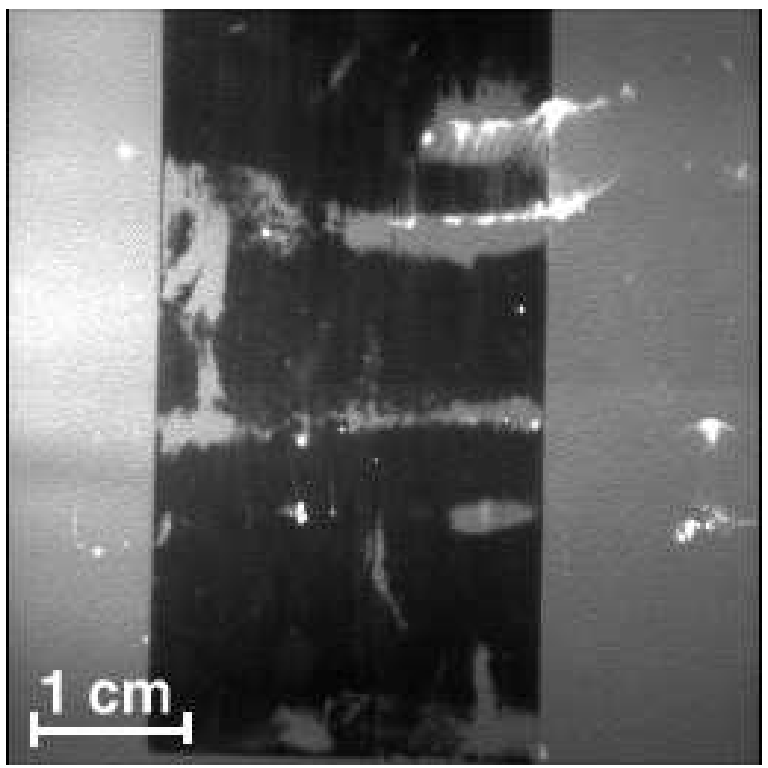

Abbildung 10.1: Typisches Reinigungsergebnis eines mit wasserfestem Stift bemalten Objektträgers im Ultraschallreaktor. Abgereinigte Stellen erscheinen grau, Blasenstrukturen weiß.

Abbildung 10.2 zeigt einen Objektträger von der Seite. Hier wurde eine höhere räumliche Auflösung gewählt, sowie eine Beleuchtung im Durchlicht mit einer Belichtungszeit von etwa $5 \mu \mathrm{s}$. Die Blasen erscheinen dunkel vor dem grauen Hintergrund. Es bildet sich eine Jellyfish-Struktur auf der Wand, die durch die Glaswand wenig gestört zu sein scheint (a). Aufgrund der technisch bedingten Frequenzmodulation des Anregungssignals kann die Beleuchtung nicht phasenstarr synchronisiert werden, deshalb erscheinen die Blasen in den gegenphasig schwingenden Lagen der Struktur von Bild zu Bild unterschiedlich groß. Die Farbe ist auf der linken Seite aufgetragen. Diese löst sich im Bereich der Blasenaktivität ab, zu erkennen an der dunkleren Trübung des Wassers in der entsprechenden Region. Die beiden Seiten werden jedoch von getrennten Blasenquellen gespeist. Insgesamt sind die Blasenstrukturen auf der bemalten Seite stärker besetzt. Während die Kavitationsstruktur auf der linken Seite für die Dauer der Aufnahme, die etwa 5 s umfasst, sichtbar ist, sind auf der rechten Seite bisweilen keine oder nur sehr wenige Blasen zu sehen. Abbildung 10.3 zeigt zwei typische Ereignisse im Kavitationsfeld. Zum einen die Vereinigung eines spontanen Clusters (durch den linken Pfeil markiert) mit der Jellyfish-Struktur. Zum anderen die Kollision eines spontanen Clusters mit der Grenzfläche (rechter Pfeil). Die Vereinigung des linken Clusters mit der Struktur erfolgt stetig. Bemerkenswert ist, dass sich verhaltnismäßig viel Farbe zwischen den Lagen der Jellyfish-Struktur befindet. Dies ist der Bereich, der kaum abgereinigt wird, die Farbe muss also durch Strömun- 
gen von den Orten der Ablösung hereingetragen worden sein. Der Aufprall des rechten Clusters mit der Wand führt zu einer starken Verformung der vorher nahezu sphärischen Struktur. In Bild (b) ist das Kollisionsereignis im Detail gezeigt. Aus den zwei Bildern vor dem Aufprall lässt sich eine Geschwindigkeit des Clusterschwerpunktes von etwa $0.5 \mathrm{~m} / \mathrm{s}$ ermitteln. Beim Aufprall wird der Cluster flachgedrückt, ähnlich wie bei einem Wassertropfen löst sich in der Mitte ein Fragment. Danach schwingt der Cluster wieder auf. Kollisionen mit der Wand sind auf der linken Seite nicht zu beobachten, dort werden die Cluster in die Kavitationsstruktur gezogen. Vermutlich sind hier die sekundären Bjerkneskräfte aufgrund der stärkeren Population der Struktur größer.
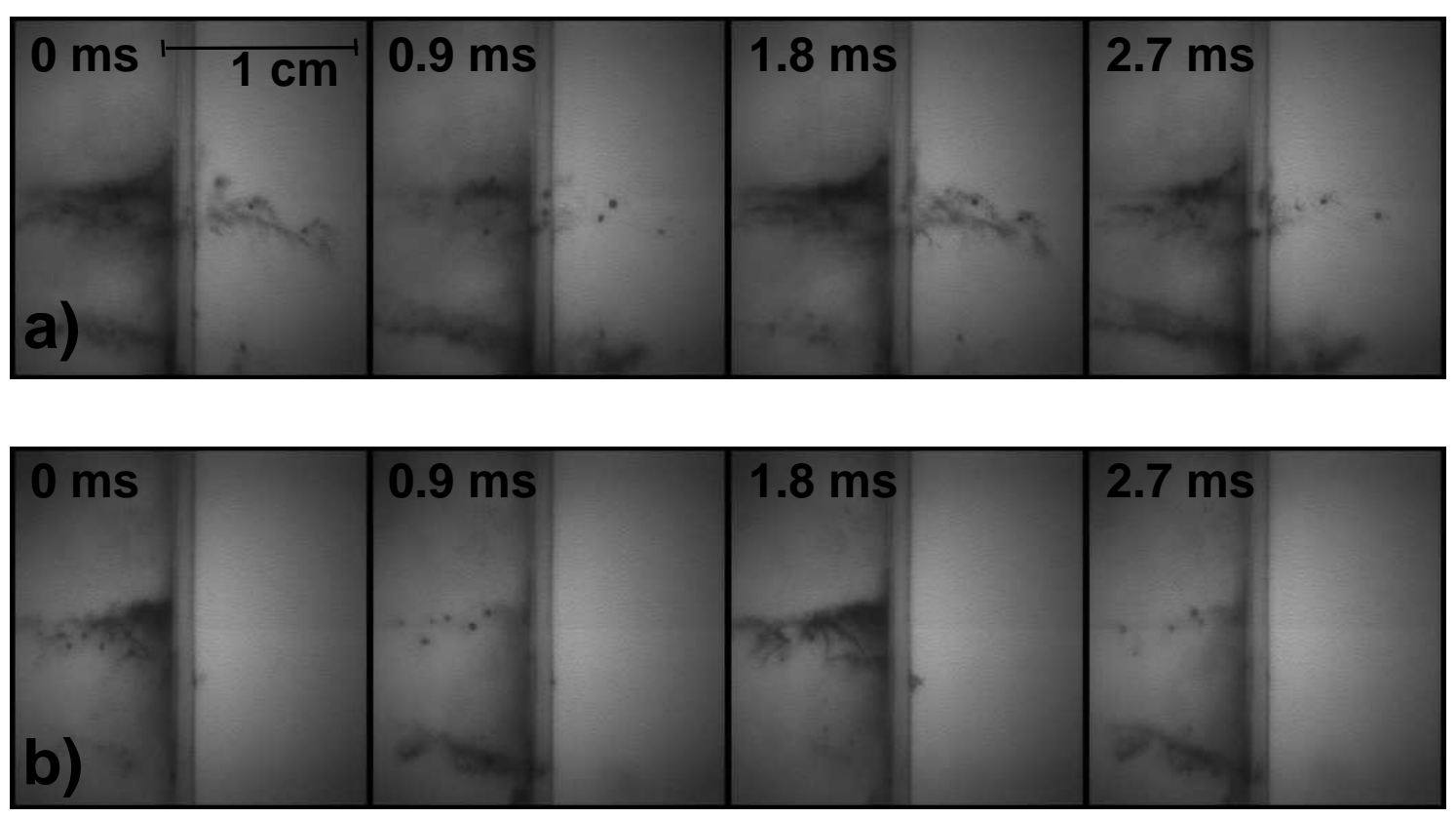

Abbildung 10.2: Jellyfish-Strukturen an einem bemalten Objektträger, Seitenansicht. Die bemalte Seite befindet sich links im Bild. Die Beleuchtung von etwa $5 \mu \mathrm{s}$ ist bei freilaufender Phase, deshalb erscheinen die gegenphasig schwingenden Phasen der Jellyfish-Struktur unterschiedlich stark. Die Zeit ist jeweils relativ zum ersten Bild der Sequenz. Komplette Jellyfish-Struktur (a), Bildung eines halben Struktur auf der bemalten Seite (b).

In Abbildung 10.4 ist ein Zwischenstadium zwischen Vereinigung mit einer Kavitationsstruktur und einer Wandkollision abgebildet. Rechts im Bild befindet sich die Glaswand, wieder von der Seite. Diesmal wurde nur ein $1 \mathrm{~cm}$ breiter Längsstreifen aus dem Objektträger herausgeschnitten, um den gesamten Bereich der Glaswand im Bereich der Schärfentiefe zu haben. Die Farbe ist wieder auf der linken, dem Cluster zugewandten Seite. Die Wand ist vollständig von 

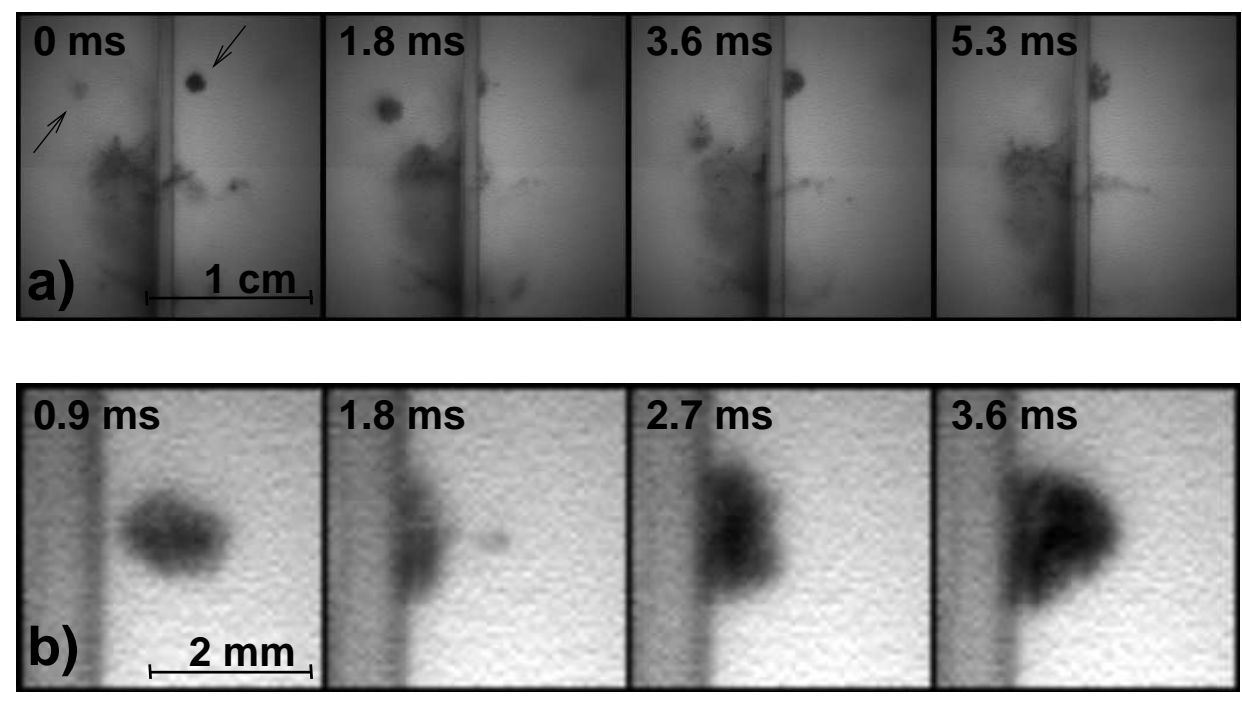

Abbildung 10.3: Entwicklung spontan entstehender Cluster in Anwesenheit einer bemalten Glasplatte (siehe Abbildung 10.2). Sequenz (a): Vereinigung eines Clusters (linker Pfeil) mit der Kavitationsstruktur und Kollision eines Clusters (rechter Pfeil) mit dem Objektträger. Eine Detailaufnahme der Wandkollision ist in Sequenz (b) wiedergegeben. Die Zeitangabe ist relativ zum ersten Bild von Sequenz (a).

Blasen bedeckt, die sich nach oben zu bewegen scheinen. Die farbfreie Seite des Objektträgers ist diesmal völlig blasenfrei. Der Cluster hat einen Maximalradius von etwa $1 \mathrm{~mm}$ und scheint aus einer Vielzahl von Blasen zu bestehen, die analog zum stationären Cluster im Kollaps auf eine oder wenige Blasen zusammenzufallen scheinen (hier nicht gezeigt). Der Cluster bewegt sich zuerst nicht von der Stelle und wird erst etwa 12 Millisekunden nach seinem Erscheinen in Richtung Wand gezogen. Die Kollision fällt dementsprechend eher als stetige Vereinigung mit den Wandblasen aus. Die entstehende Anhäufung an Blasen wandert weiter nach oben.

In Abbildung 10.5 ist die Entwicklung des Kavitationsfeldes nach Einschalten des Reaktors zu sehen. Sequenz (a) zeigt die erste dreiviertel Sekunde nach dem Einschalten bei $t=0$. Zu Beginn schwingen auf der Oberfläche befindliche Blasen stark auf und formieren sich zu Clustern. An verschiedenen Stellen entstehen Blasen. Oft bildet sich zumindest zeitweise ein kontinuierlicher Blasenstrom, der in einem Cluster endet. Diese Strukturen können sehr ausgedehnt werden, wie in der Aufnahme einer halben Sekunde nach dem Einschalten zu sehen ist. Der Ursprung der zwei Strukturen liegt jeweils etwa in der Ebene maximaler Schalldruckamplitude, dies ist vermutlich die Ursache für die starke Blasenent- 


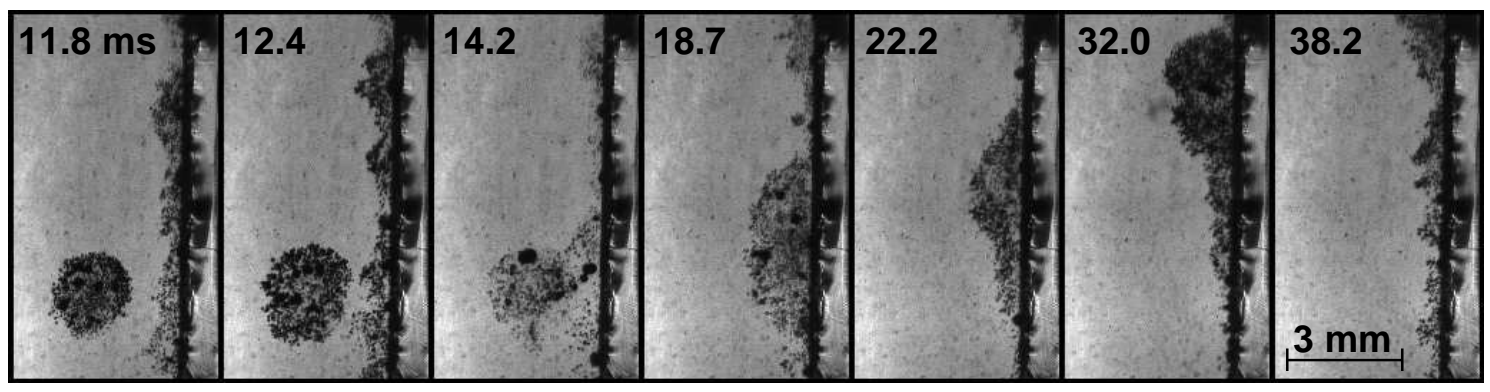

Abbildung 10.4: Vereinigung eines spontan entstehenden Clusters mit Blasen, die sich auf der bemalten Seite der Glasplatte befinden.

wicklung. Rechts neben der unteren Struktur beginnt sich eine Jellyfish-Struktur $\mathrm{zu}$ formieren. In Bezug auf die Reinigung ist $\mathrm{zu}$ bemerken, dass die Blasenquelle der unteren Struktur bei der Translation nach oben eine abgereinigte Spur hinterlässt, markiert mit einem weißen Pfeil in Bild $4(\mathrm{t}=0.75 \mathrm{~s})$. Typisch ist, dass die Blasenquellen zeitweilig abreißen. An den gleichen oder benachbarten Stellen entstehen nach kurzer Zeit oft neue Quellen. Diese verursachen wenig ausgedehnte punkt- oder strichförmige Reinigungsereignisse, während ein Cluster, wenn er auf der Oberfläche aktiv ist, größere Flächen abreinigen kann. Sequenz (b) gibt den gleichen Reinigungsvorgang zu einem späteren Zeitpunkt wieder. Die von der Blasenquelle zu Beginn verursachte Spur hat sich verlängert, rechts daneben hat sich eine weitere Spur gebildet. Die abgereinigten Flächen werden hier hauptsächlich durch die Clusterstrukturen verursacht, wie zum Beispiel an der mit einem weißen Kasten markierten Stelle. Ein reguläres Muster der Reinigung ist nicht oder noch nicht zu sehen, allerdings sind auch hier Bereiche um den Schalldruckknoten (siehe die vorhandenen Jellyfish-Strukturen im unteren Drittel und im oberen Bereich, etwas verdeckt von der Zeitangabe) von der Reinigung weitgehend ausgespart. 

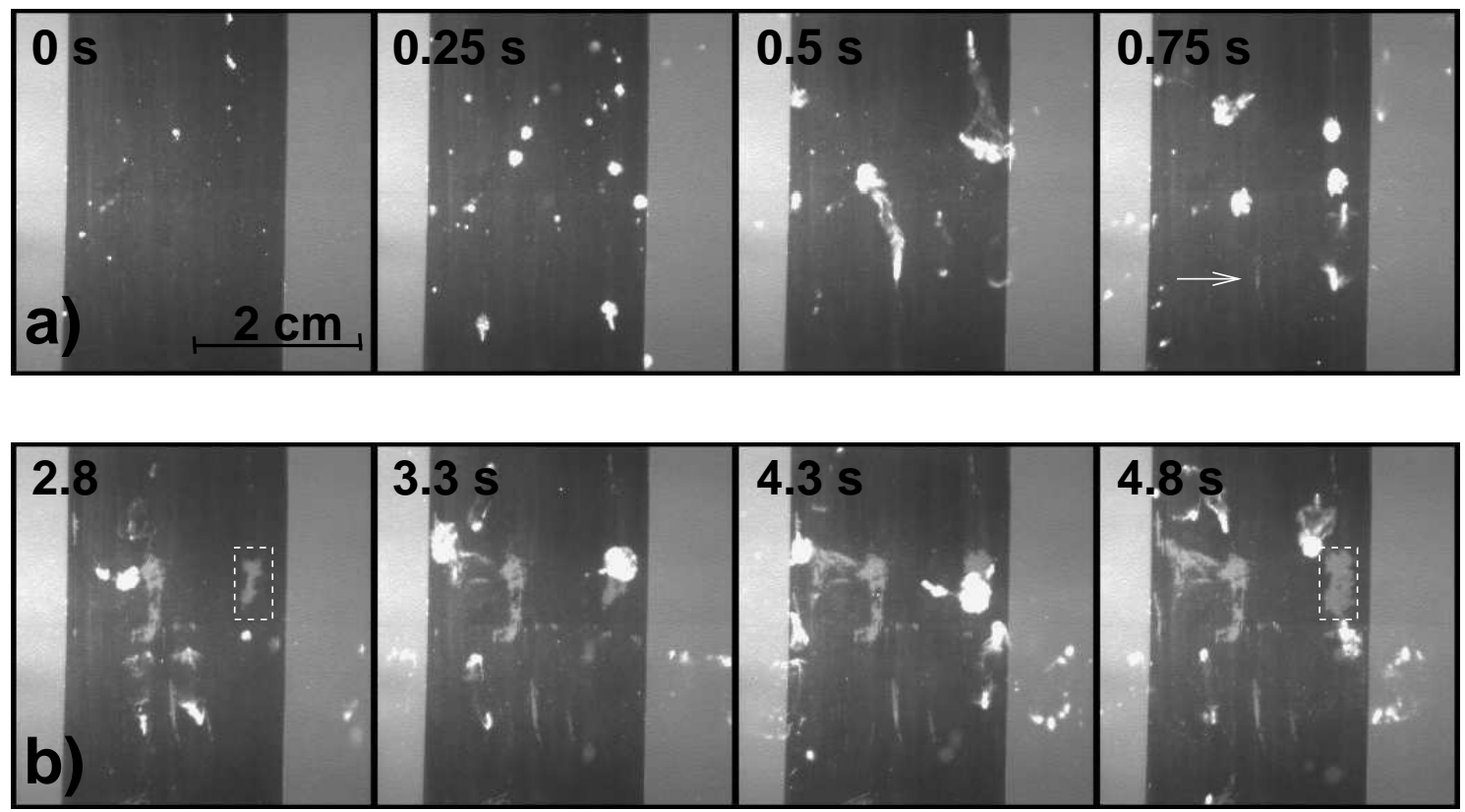

Abbildung 10.5: Entwicklung des Kavitationsfeldes direkt nach dem Einschalten des Ultraschalls (a) und etwa $250 \mathrm{~ms}$ später (b) in Anwesenheit einer bemalten Glasplatte im Ultraschallreaktor.

\subsection{Erosion von Aluminiumfolie}

Zur Untersuchung der Kavitationserosion wird Aluminiumfolie (ca. 7 x $10 \mathrm{~cm}$ ) mit Doppelklebeband auf einem Aluminiumrahmen befestigt und ins Zentrum des Ultraschallreaktors gebracht. Nach etwa 1 Minute findet kaum mehr Erosion statt, es bildet sich auch hier ein charakteristisches Streifenmuster, wiedergegeben in Abbildung 10.6. In der Mitte ist eine Jellyfish-Struktur (weiß) zu sehen. Die Breite der Aluminiumstege korrespondiert in etwa mit der vertikalen Ausdehnung der Jellyfish-Struktur. Die Schalldruckverteilung wird mit einem Hydrofon gemessen, genaue Werte sind wegen der Kavitation nicht zu bestimmen, jedoch können Minima und Maxima bestimmt werden. Diese sind in der Abbildung links durch horizontale weiße Striche wiedergegeben. Dicke Striche bezeichnen die Orte maximalen Schalldrucks, dünne Striche Orte minimalen Schalldrucks. Analog zu den Reinigungsergebnissen wird auch hier im Bereich der Knotenebenen die Grenzfläche kaum beeinträchtigt, während die Alufolie am Ort der Schalldruckmaxima völlig wegerodiert ist.

Abbildung 10.7 zeigt den Beginn der Kavitationserosion. Um den Einfluss der Blasenquellen zu untersuchen, wurde die Aluminiumfolie einmal in den bereits 


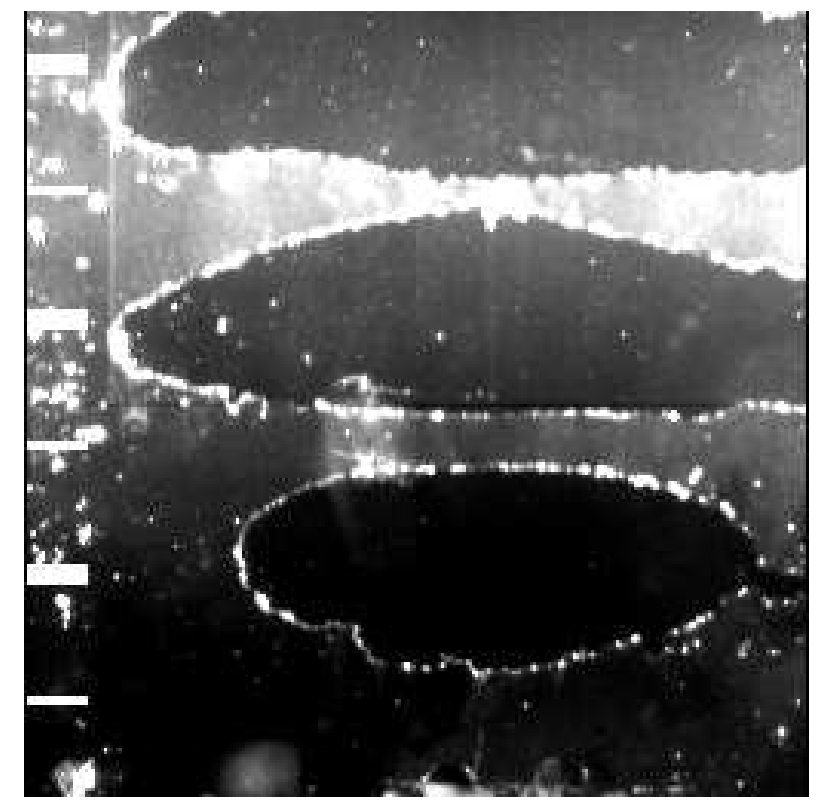

\begin{abstract}
Abbildung 10.6: Erodierte Aluminiumfolie nach etwa 1 Minute im Zentrum des Ultraschallreaktors. In den Knotenebenen des Schallfeldes verbleibt die Folie weitgehend unberührt, an Orten hoher Schalldruckamplitude ist die Folie völlig wegerodiert.
\end{abstract}

laufenden Reaktor eingebracht (a) und in einem zweiten Experiment der Reaktor nach dem Einbringen der Alufolie eingeschaltet (b). Das Erosionsmuster zeigt deutliche Unterschiede. Wird die Aluminiumfolie in das Kavitationsfeld eingebracht, nachdem sich bereits Jellyfish-Strukturen gebildet haben, findet die Erosion an deren oberen Rand statt. Im Schalldruckbauch findet keine Erosion statt. Befindet sich bei Aktivierung des Ultraschalls die Folie bereits im Reaktor, bilden sich Blasenstruktur, vornehmlich clusterartige Strukturen im Schalldruckbauch und erzeugen dort Erosion. In beiden Fällen sind die Orte von Blasenaktivität im Vergleich zur bemalten Glasplatte nahezu konstant, wenn auch an verschiedenen Orten. Dies zeigt, dass die Anfangsverteilung der Blasenquellen hier einen entscheidenden Einfluss auf die Strukturbildung hat.

\title{
10.3 Kavitationserosion an Weißblech
}

Bei Erosionsexperimenten bildet sich bei Einbringen eines Stück Weißblech in dem Reaktor eine an einer Störstelle angesiedelte Kavitationsstruktur. Feiner weißer Nebel wird ausgestoßen, der in einer festen Bahn die Störstelle zu verlassen scheint („smoker“-Struktur). Dazwischen bilden sich immer wieder größere Blasen 

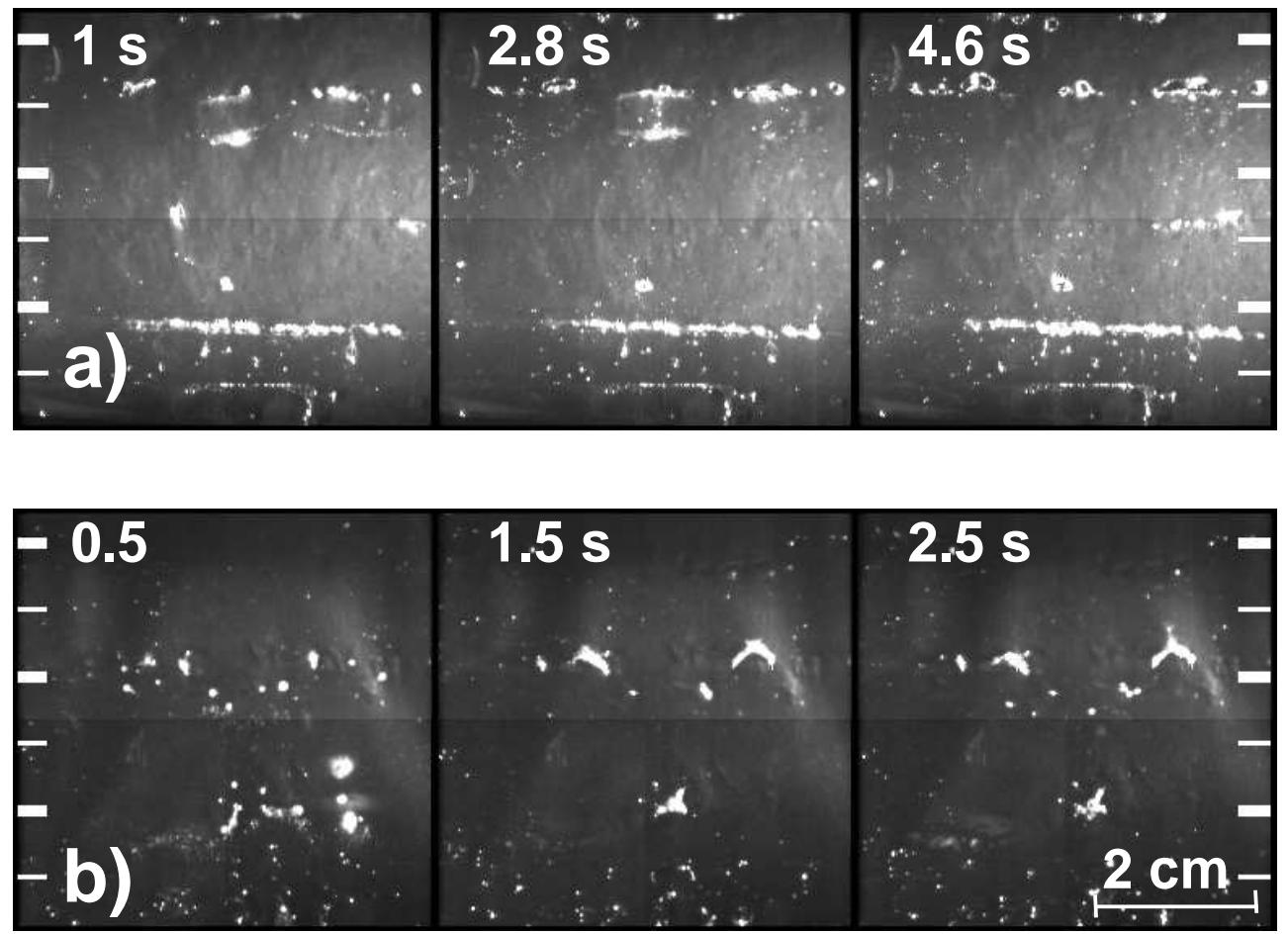

Abbildung 10.7: Kavitationserosion an Aluminiumfolie. Die Folie wird ins laufende Kavitationsfeld gebracht (a), der Ultraschall wird nach der Positionierung der Alufolie eingeschaltet (b).

oder Blasencluster. Der Nebel ist deutlich feiner als die bei den Reinigungsprozessen auftauchenden Strukturen (siehe Skalierung), die Struktur ist sehr ortsstabil. Bei der Erosion der Alufolie konnte eine solche Struktur nicht beobachtet werden. Ähnliche Strukturen bilden sich auch auf dem Stahlboden des Reaktors, der an den eng begrenzten Stellen deutliche Erosionsspuren aufweist.

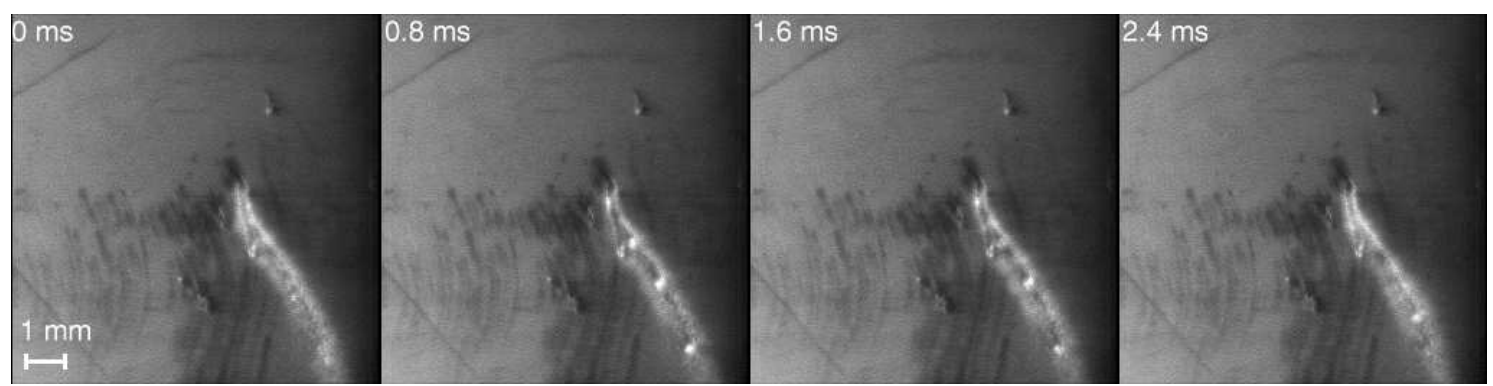

Abbildung 10.8: Kavitationsstruktur auf einem Stück Weißblech. 


\section{Kapitel 11}

\section{Zusammenfassung}

Im Rahmen dieser Arbeit wurden Experimente zu akustischer Kavitation durchgeführt, die von Einzelblasen im Schalldruckbereich von wenigen $\mathrm{kPa}$ bis zu Vielblasenstrukturen bei Schalldruckamplituden von $500 \mathrm{kPa}$ reichen. Bereits das vergleichsweise überschaubare Einzelblasensystem zeigt ein komplexes raumzeitliches Verhalten.

Es wurde die Translationsbewegung im Schallfeld theoretisch, numerisch und experimentell unter besonderer Berücksichtigung der viskosen Widerstandskraft untersucht. Ein analytischer Ansatz hat gezeigt, dass die genäherte Mittelung der Bewegungsgleichung auch für schwach schwingende Blasen problematisch sein kann. Der Vergleich zwischen experimentellen und numerischen Trajektorien ergab, dass keine der einfachen Näherungen für die Reibungskraft die Blasenbewegung im untersuchten Parameterbereich zufriedenstellend beschreibt. Die Messungen sind jedoch mit der Theorie konsistent und geben einen Hinweis darauf, dass Blasen im starken Schallfeld durch die Näherung bei hoher Reynoldszahl beschrieben werden können.

Untersuchungen zur Oberflächeninstabilität von Einzelblasen haben den Zusammenhang zwischen irregulärer Translationsbewegung einer Blase und der unsymmetrischen Abspaltung von Mikroblasen gezeigt. Die Mikroblasen können dabei auf Geschwindigkeiten von bis zu $20 \mathrm{~cm} / \mathrm{s}$ beschleunigt werden. Die Anregung von Oberflächeninstabilitäten konnte auch bei der Einzelblasensonolumineszenz beobachtet werden. Durch simultane Messung der Lichtemission, der Radial- sowie der Translationsdynamik konnte das Emissionsverhalten mit der Blasendynamik korreliert werden. Eine überraschende Erweiterung des Parameterbereichs, in dem SBSL existiert, ist die Erzeugung von Einzelblasensonolumineszenz (SBSL) in luftgesättigtem Wasser. Voraussetzung hierfür ist ein blasenquellenfreies Schallfeld. In Anwesenheit von Blasenquellen konnten bei gleicher Schalldruckamplitude Streamerstrukturen erzeugt werden. 
Durch gezieltes Positionieren der Blasenquellen konnten ein- und zweidimensionale Streamerfiguren erzeugt werden. Die Translationsdynamik des eindimensionalen Streamers wurde über den gesamten Stabilitätsbereich von $p_{a}=100-200 \mathrm{kPa}$ untersucht. Dabei konnte insbesondere die Bildung einer Clusterstruktur beobachtet werden, die eine zentrale Rolle in der akustischen Kavitation zu spielen scheint. Ihr Verhalten im Schallfeld legt nahe, dass die Clusterstruktur in ihrer Translationsdynamik im Rahmen der Einzelblasentheorie behandelt werden kann. Sie entsteht aus Streamerstrukturen oder kann einzeln injiziert werden. Sie bildet sich aber auch spontan im Schalldruckbauch bei hohen Anregungsamplituden und ist damit die Brücke zu sehr starken Kavitationsfeldern. Dort entstehen aus den Clustern sogenannte Jellyfish-Strukturen, die eine charakteristische Doppellagenstruktur um die Schalldruckknotenebenen ausbilden. Der Schalldruckbauch wird dabei von Blasen entvölkert. Diese Blasenfigur wurde im Rahmen der Arbeit untersucht und charakterisiert. Die Reproduktion der - üblicherweise in Hochleistungs-Ultraschallbädern auftretenden - Figur im kleinen Laborresonator bestätigt die Aussagen über die Entstehung und den Charakter der Jellyfish-Struktur. Diese kann mit Reinigungs- und Erosionsprozessen in Verbindung gebracht werden. Hochgeschwindigkeitsaufnahmen zeigen hier die Bedeutung von Blasenquellen für die Entwicklung des Kavitationsfeldes.

Die auf einer Zeitskala von Sekunden stattfindende Strukturbildung konnte auch im akustischen Kavitationsspektrum beobachtet werden. Durch simultane Aufnahme der Blasenstrukturen und des akustischen Spektrums konnte die zeitliche Korrelation von Strukturbildungsprozessen und Periode-2-Schwingungen gezeigt werden. Untersuchungen in einer Ultraschallwanne legen dabei nahe, dass in Hochleistungs-Ultraschallgeräten die Subharmonische insbesondere von Blasen im Nahfeld der Schallwandler erzeugt wird. Bei niedrigeren Schalldruckamplituden spielen vor allem die höheren Harmonischen eine wichtige Rolle. Hier konnte gezeigt werden, dass die Clusterstruktur mit der Entstehung der zweiten Harmonischen zusammenhängt. Diese kann so stark angeregt werden, dass die Cluster sich im Schallfeld in einer durch höhere Resonatormoden vorgegebenen Struktur anordnen. Auch bei schwach angeregten Blasen können Translationsbewegungen beobachtet werden, die auf die Wechselwirkung mit höheren Harmonischen im Schallfeld hindeuten. Doch nicht nur durch Kopplung in andere Frequenzen und Dämpfung beeinflusst die Kavitation das Schallfeld. Bereits eine schwach angeregte Einzelblase kann das Schwingungsverhalten des Gesamtsystems stark beeinflussen, wie Resonanzmessungen in der Nähe der Grundmode gezeigt haben. 


\section{Kapitel 12}

\section{Ausblick}

Die im Rahmen dieser Arbeit durchgeführten Untersuchungen illustrieren die Komplexität der akustischen Kavitation. Die gefundenen Ergebnisse werfen eine Reihe neuer interessanter Fragen auf, die für das physikalische Verständnis der in akustischen Kavitationsfeldern auftretenden Phänomene wichtig sind.

Die akustischen Messungen zeigen, dass Blasen entscheidend auf das Schallfeld zurückwirken, so dass dieser Effekt auch bei Einzelblasen nicht vernachlässigt werden kann. Dies stellt zum einen neue Anforderungen an die Modellbildung, da eine Reihe der beobachteten Phänomene wesentlich durch die bidirektionale Kopplung mit dem äußeren Schallfeld bestimmt sind. Diese Phänomene sind insbesondere die Resonanzverschiebung des Gesamtsystems, der horizontale Bewegungsanteil aufsteigender Einzelblasen, die Korrelation zwischen Schalldruck und Clusterposition im eindimensionalen Streamer, die Orientierung der Clusterstruktur im Schallfeld und die instabile zweidimensionale Lichtenbergfigur. Zum anderen ist die physikalische Ursache der auftretenden Phänomene zu untersuchen. Durch Resonanzmessungen in Anwesenheit von gecoateten Blasen könnte die Wechselwirkung zwischen Resonator und Blase gezielter untersucht werden, da das diffusive Blasenwachstum unterdrückt wird.

Die Schallemission höherer Harmonischer kann auch bei Einzelblasen gemessen werden, wobei sich eine Abhängigkeit der Linienstärke von der Blasengröße zeigt. Es stellt sich die Frage, ob auch nichtlineare Resonanzen der Blase im Spektrum gemessen werden können, oder ob die Blase oberflächeninstabil wird.

Der recht abrupte Positionswechsel der Clusterstruktur in andere Gleichgewichtslagen ist ebenfalls ein interessanter Untersuchungsgegenstand. Die Schallfeldverteilung und die Ursache für die sehr starke Anregung der zweiten Harmonischen sollte näher untersucht werden. 
Insgesamt ist die Clusterstruktur vermutlich von großer Wichtigkeit für die Strukturbildung in akustischen Kavitationsfeldern, da sie in einem sehr weiten Parameterbereich auftritt und spontan entstehen kann. Ihre detailierte raumzeitliche Struktur und die Bedingungen für ihre Stabilität sind von besonderem Interesse. Außerdem wäre zu untersuchen, inwieweit die spontan entstehenden Cluster die gleichen Charakteristika wie die stationären aufweisen.

Die Entwicklung dimensionsreduzierter Streamerfiguren ist für die Untersuchung grundlegender Mechanismen der Blasendynamik hilfreich, da sowohl die Bewegungsrichtung der Blasen als auch die räumliche Ausdehnung der Struktur vorgegeben ist. Im eindimensionalen Streamer können Wechselwirkungen zwischen den Blasen sowie die Entstehung der Clusterstruktur untersucht werden. Die gewonnenen Daten würden gute Vergleichsmöglichkeiten mit numerischen Simulationen bieten. Durch sorgfältige Präparation der Blasenquelle könnten eine lumineszierende Einzelblase gezielt gestört und kontinuierlich in eine Streamerstruktur überführt werden. Erste Experimente zeigen, dass dies möglich ist. An einem solchen System kann der Übergang von SBSL zu MBSL untersucht werden. In der Clusterstruktur konnte im Vorexperiment zwar keine Lichtemission gemessen werden, doch müssten systematische Messungen mit einem empfindlicheren Photomultiplier den Befund bestätigen oder widerlegen.

Um den Einfluss der sekundären Bjerkneskraft zu bestimmen, kann mithilfe von zweidimensionalen Streamern der Verlauf der Trajektorien bei Annäherung von zwei Blasenquellen untersucht werden. Sukzessive können die Dimensionalität der Streamerstruktur erhöht und die jeweiligen Eigenschaften bestimmt werden.

Insgesamt bietet der Bereich der Blasenentstehung ein breites Forschungsgebiet. Im Experiment versiegten Blasenquellen teilweise schnell, teilweise wurden bei gleichen äußeren Parametern so viele Blasen erzeugt, dass sich keine stabile Streamerstruktur aufbauen konnte. Diese Phänomene sind auch für technische Anwendungen wichtig. Hier stellt sich insbesondere die Frage der Steuerbarkeit der vorhandenen Blasenquellen.

Die Charakterisierung der auftretenden Kavitationsfiguren ist nicht abgeschlossen. Relativ wenig untersucht wurde im Rahmen dieser Arbeit die "Smoker“Struktur, die an Grenzflächen zu beobachten ist. Es stellt sich unter anderem die Frage, ob die Jellyfish-Struktur ähnlich wie ein Streamer auf Blasenquellen angewiesen ist, oder ob sie sich selbst erhält und separat erzeugt werden kann. In diesem Fall könnte ihr Verhalten gezielt untersucht werden, was im normalen Kavitationsfeld aufgrund der Vielzahl der auftretenden Strukturen und Phänomene nicht möglich ist. 
Ein in dieser Arbeit nur am Rande behandeltes Phänomen ist das Anschwingen einer tieffrequenten Schwerewelle, die in allen verwendeten Resonatoren beobachtet werden konnte. Die physikalische Ursache dieses nichtlinearen Effektes und die Wechselwirkung zwischen Schwerewelle und Kavitationsfeld sind noch ungeklärt.

Bezüglich der hydrodynamischen Eigenschaften der Translationsbewegung ist die experimentelle Bestimmung der Trajektorien im starken Schallfeld notwendig. Hierbei ist insbesondere die Erzeugung der Blase problematisch, da Elektroden einen großen Einfluss im Nahfeld der Blase haben. Es wurden in Zusammenarbeit mit Reinhard Geisler und Philipp Koch bereits erste Messungen an mit dem Femtosekundenlaser erzeugten Blasen im Schallfeld durchgeführt. Schwierigkeiten treten vor allem aufgrund der Fragmentation der Blase während der Translationsbewegung auf. Dies deutet darauf hin, dass Oberflächenverformungen nicht vernachlässigt werden können.

Bei den Translationsexperimenten für diese Arbeit wurden zum Teil mehrere Blasen erzeugt. Liefen diese hintereinander her, hatten sie eine deutlich höhere Aufstiegsgeschwindigkeit als die Einzelblasen. Dies konnte im Rahmen dieser Arbeit nicht näher untersucht werden, ist aber insofern für Kavitationsstrukturen interessant, als dort auch oft mehrere kleine Blasen im Nachlauf einer etwas größeren beobachtet werden. Hier zeigen sich vermutlich die Grenzen eines auf Einzelblasenbewegungen basierenden Modells. Eine Untersuchung der Translation dieser Blasenketten könnte zum besseren Verständnis des Verhaltens von Blasen in Streamerstrukturen beitragen. 



\section{Anhang A \\ Beschreibung der verwendeten Resonatoren}

\section{A.1. Glaswürfel}

\section{A.1.1. Beschreibung des Resonators}

Als Resonator wird eine kommerzielle quaderförmige Fluoreszenzküvette ( Hellma 704.003) aus optischem Glas verwendet. Als Schallwandler wurde eine ringförmige, vertikal polarisierte Piezokeramik (pi ceramics, PIC 151) mittig am Resonatorboden veklebt (Uhu endfest 300). Die Abmessungen der Bauteile betragen:

1. Glaswürfel

Innenmaße: $\mathrm{x}=\mathrm{y}=\mathrm{z}=50 \mathrm{~mm}$

Wandstärke: $2.5 \mathrm{~mm}$

2. Piezoring

Innendurchmesser: $10 \mathrm{~mm}$

Außendurchmesser: $20 \mathrm{~mm}$

Höhe: $0.5 \mathrm{~mm}$

Der Resonator weist eine hohe Güte auf. In der Grundmode, die bei etwa $24 \mathrm{kHz}$ liegt, ergeben Hydrofonmessungen eine Halbwertsbreite von weniger als $50 \mathrm{~Hz}$. Da das Hydrofon (oder ihm anhaftende Blasen) den Resonator signifikant dämpfen (siehe unten), gibt dies nur eine untere Grenze für die Güte des Resonators an. Da eine leichte Verstimmung zu einer sehr starken Änderung der Schalldruckamplitude führt, wird der Resonator zusätzlich gedämpft. Zum einen wird der Resonatorboden mit Ausnahme des Piezoringes auf Moosgummi gelagert. Zum 
anderen wird die Küvette mit einem hartgummibeschichteten Metalldeckel geschlossen. Als Zugang ist ein Loch von 1cm Durchmesser in der Mitte des Deckels angebracht. Die Güte des gedämpfen Systems beträgt jetzt etwa $Q \approx 400$, die Halbwertsbreite liegt bei etwa $60 \mathrm{~Hz}$ (gemessen mit dem Vibrometer, die Hydrofonmessung ergibt eine Halbwertsbreite von $80 \mathrm{~Hz}$ ).

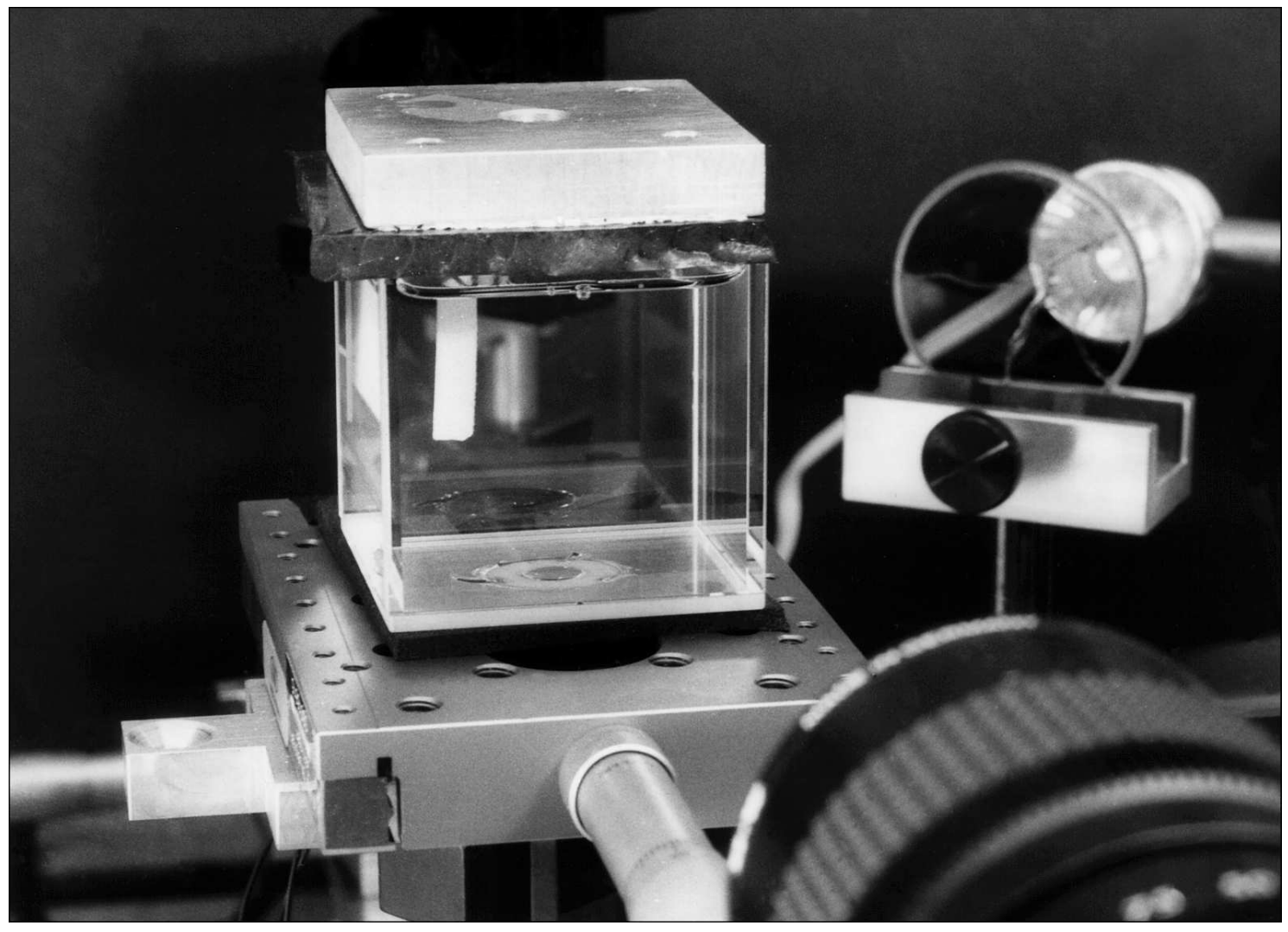

Abbildung A.1.: Aufnahme des Glasquaders in der verwendeten Form. Aufnahme G. Kirschmann-Schröder.

\section{A.1.2. Bestimmung der Resonatoreigenschaften}

\section{Hydrofonmessung}

Zur Bestimmung der Resonatoreigenschaften wird die Schalldruckamplitude im Küvettenzentrum um die Grundresonanzfrequenz (1,1,1-Mode) bestimmt. Das kleinste im benötigten Frequenzbereich kalibrierte Hydrofon, das zu Verfügung stand, hat einen Durchmesser von $5 \mathrm{~mm}$ (Reson TC 4038). Dieses wird in die Mitte der Küvette gebracht und auf maximales Schallsignal feinjustiert. Bei fester Amplitude des Funktionsgeneratorsignals wird die Frequenz in einem Bereich um 
die Resonanzfrequenz variiert, das Hydrofonsignal mit einem 16-bit A/D Wandler bei einer Samplingfrequenz von $256 \mathrm{kHz}$ abgetastet und 4096 Werte $(16 \mathrm{~ms})$ gespeichert. Das Eingangssignal wird mit einer Grenzfrequenz von $100 \mathrm{~Hz}$ hochpassgefiltert. In Abbildung A.2 a) ist die Schalldruckamplitude dargestellt. Da der Schallwandler ohne Impedanzanpassung angesteuert wird, ist die Spannung am Schallwandler über den gesamten Frequenzbereich direkt proportional zur Spannungsamplitude des Funktionsgenerators, der hier als Parameter verwendet wird. Deutlich zu sehen ist eine amplitudenabhängige Resonanzkurve des Systems. Zum

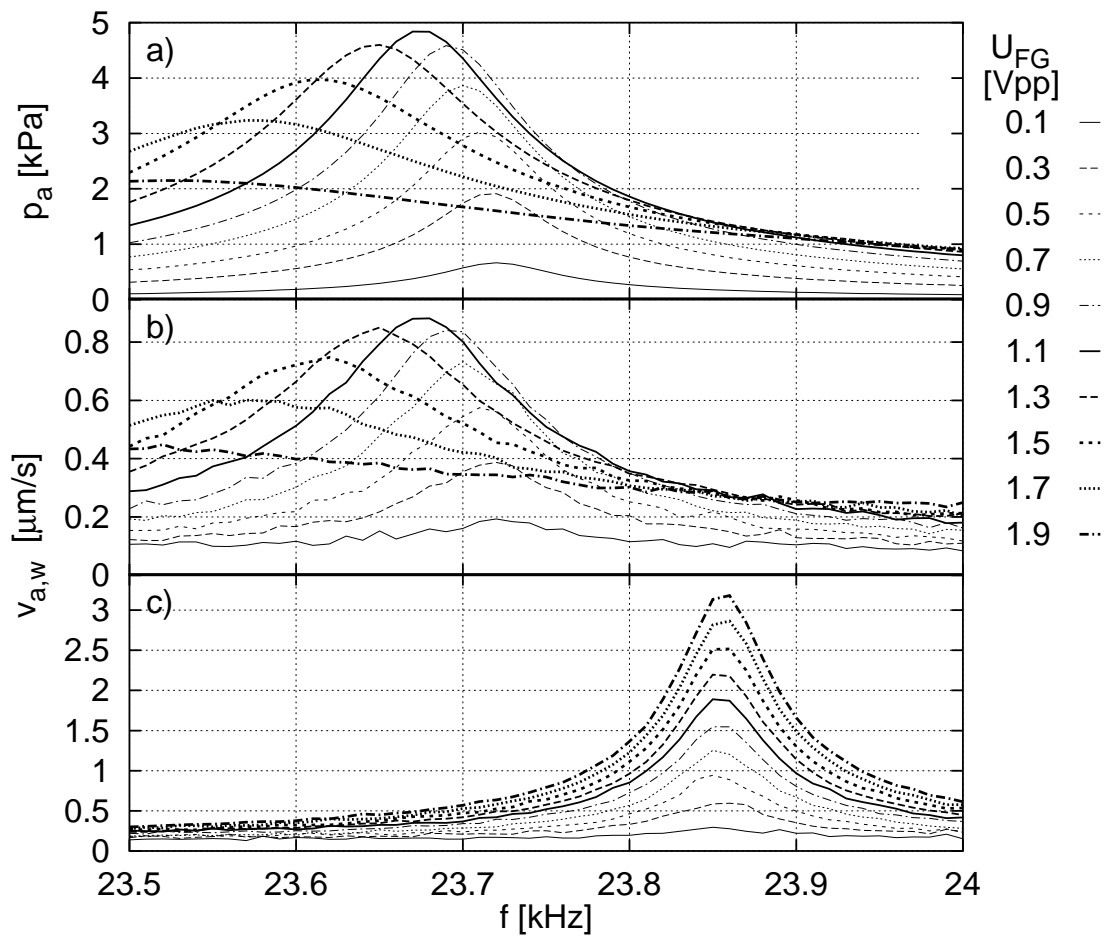

Abbildung A.2.: Resonanzkurve des Glasquaders bei verschiedenen Anregungsamplituden $U_{F G}$. a) Hydrofonmessung im Zentrum des Quaders, b) Simultan zu Messung a) durchgeführte Vibrometermessung an der Quaderwand, c) Vibrometermessung in Abwesenheit des Hydrofons.

einen wird der Resonator mit zunehmender Anregungsamplitude gedämpft. Die Güte von $Q \approx 300$ bei $U_{F G}=0.5 \mathrm{Vpp}$ fällt auf $Q \approx 140$ bei einer Anregungsamplitude von $U_{F G}=1.5 \mathrm{Vpp}$. Die Dämpfung sollte aber lediglich eine Verschiebung des Schalldruckmaximums im Bereich von etwa $1 \mathrm{~Hz}$ verursachen. Betrachtet man das System als einfaches mechanisches Masse-Feder-System, so wird eine Verringerung der Resonanzfrequenz durch eine Erhöhung der Masse oder durch eine Verkleinerung der Federkonstante verursacht. Die Masse sollte im wesentlichen konstant sein, deshalb liegt eine Änderung der Nachgiebigkeit des Systems nahe. 
Diese kann durch auf dem Hydrofon haftende Mikroblasen verursacht werden. Sichtbare Blasen wurden vor der Messung von den Resonatorwänden und vom Hydrofon entfernt. Die Amplitudenabhängigkeit zeigt, dass es sich dabei um ein nichtlineares Phänomen handelt, das im Rahmen eines linearen Oszillators nur qualitativ beschrieben werden kann.

\section{Laser-Doppler-Interferometrie}

Simultan mit der Hydrofonmessung wird die Auslenkungsgeschwindigkeit an einem Punkt der Wand gemessen. Der Punkt wurde so gewählt, dass er sich möglichst nah am Zentrum der Wand befindet, um eine maximal Auslenkung zu erhalten. An der Stelle wurde ein kleines Stück Reflektormaterial befestigt.

Da die Transmission des Lasers jedoch beträchtlich ist, muss der Strahl soweit vom Zentrum entfernt sein, dass er nicht mehr auf das Hydrofon trifft. Das Vibrometersignal wurde ebenfalls hochpassgefiltert und mit dem zweiten Kanal des A/D-Wandlers digitalisiert. In Abbildung A.2 b) ist der Verlauf der Geschwindigkeitsamplitude $v_{a, w}$ für die gleiche Messreihe wie in Abbildung A.2 a) dargestellt. Die Geschwindigkeitsamplitude folgt im Wesentlichen dem Hydrofonsignal, das Signal ist allerdings stärker verrauscht. Die amplitudenabhängige Resonanzverschiebung zeigt sich auch in der Geschwindigkeitsresonanz; die durch Dämpfung verursachte Verschiebung zwischen Schalldruck- und Schnellemaximum liegt hingegen unterhalb der Frequenzauflösung. Vor allem bei kleinen Amplituden ist der Rauschanteil hoch. Der Proportionalitätsfaktor zwischen Schalldruck und Wandgeschwindigkeit läuft für hohe Amplituden gegen einen konstanten Wert von etwa $5.5 \mathrm{kPa} /(\mathrm{mm} / \mathrm{s})$. Bei kleinen Amplituden fällt der Faktor auf 4, dies ist eine Folge der Vergrößerung der Geschwindigkeitsamplitude durch den Rauschanteil. Wenn das Signal-Rausch-Verhältnis groß ist, kann die Schalldruckamplitude durch das Vibrometersignal bestimmt werden. Eine Vergleichsmessung der Wandgeschwindigkeit, bei dem das Hydrofon aus dem Resonator entfernt wurde, zeigt den starken Einfluss des Hydrofons auf die Resonatoreigenschaften. In Abbildung A.2 (c) ist die Amplitude des Vibrometersignals wiedergegeben. Die höhere Resonanzfrequenz im Vergleich zu der Messung bei Anwesenheit des Hydrofons ist zum einen durch die etwas geringere Füllhöhe gegeben, da das Volumen des Hydrofons fehlt. Zum anderen ist aber die Resonanz auch bei niedriger Anregungsamplitude deutlich schärfer, und die Resonanzüberhöhung stärker, also ist auch in diesem Amplitudenbereich der Einfluss des Hydrofons relevant.

In Abbildung A.3 sind zur Übersicht die Maximalgeschwindigkeiten und Resonanzfrequenzen der Messungen mit und ohne Hydrofon zusammengefasst. 


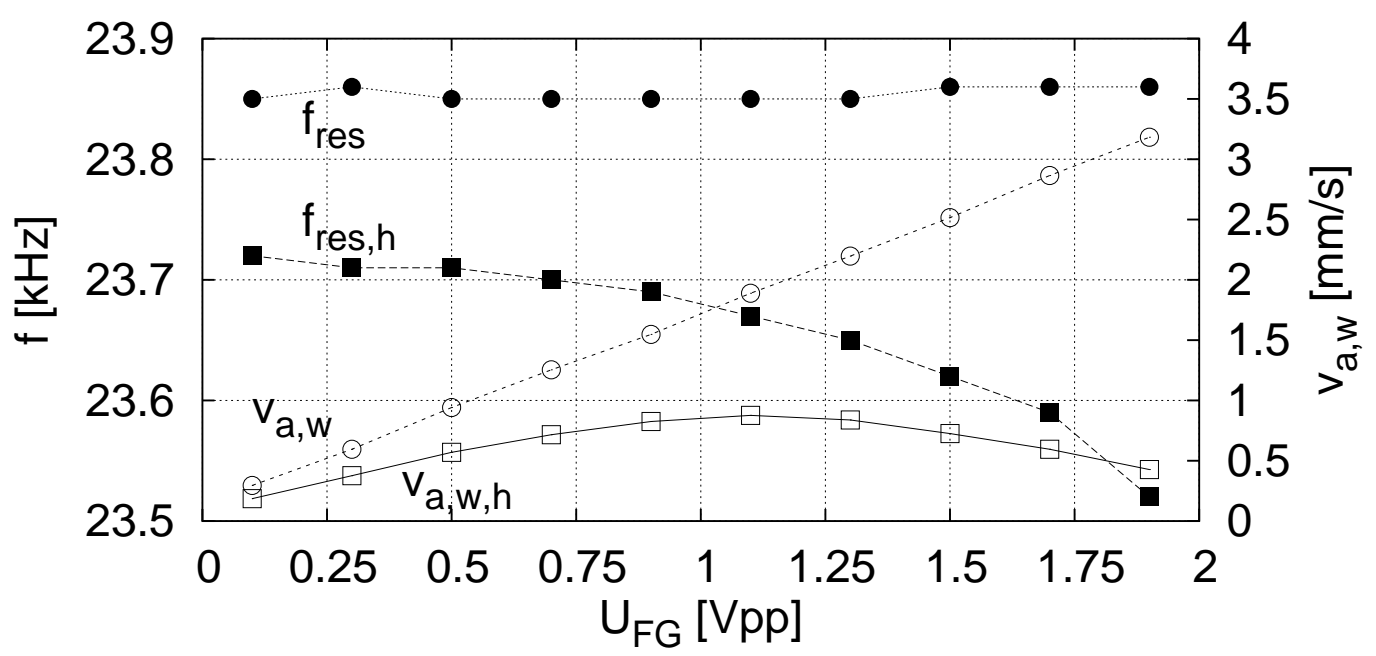

Abbildung A.3.: Amplitude der Wandgeschwindigkeit $v_{a, w}$ und Resonanzfrequenz $f_{\text {res }}$ bei verschiedenen Anregungsamplituden $U_{F G}$, mit $\left(v_{a, w, h}, f_{\text {res }, h}\right)$ und ohne Hydrofon.

Der hydrofonfreie Resonator verhält sich wie ein linearer Oszillator. Die Geschwindigkeitsamplitude steigt linear mit der Anregungsamplitude, die Resonanzfrequenz ist konstant, während bei Anwesenheit des Hydrofons die Schwingungseigenschaften stark amplitudenabhängig sind. Die Hydrofonmessung kann also zur Kalibrierung der Wandgeschwindigkeit verwendet werden, jedoch nicht zur Vermessung der Resonatoreigenschaften. Ein derart starker Einfluss des Hydrofons selber als passives System ist unwahrscheinlich. Möglicherweise befinden sich auf der Oberfläche des Hydrofons Kavitationskeime und Mikroblasen, die zu nichtlinearen Effekten führen können. Dazu passt die empirische Beobachtung, dass der Amplitudenabfall auch bei gleicher Anregungsamplitude unterschiedlich stark ausfallen kann.

\section{Frequenzspektrum}

Die Fourieranalyse der Zeitreihen zeigt das Auftreten höherer Harmonischen sowie weiterer Linien. Die Amplituden dieser Linien liegen unter 1\% der Anregungsamplitude. In Abbildung A.4 ist der hochfrequente Ausschnitt des Leistungsspektrums der Wandschwingung ohne (a) und mit Hydrofon (b) in der Resonanz bei einer vergleichbaren Geschwindigkeitsamplitude von etwa $0.8 \mathrm{~mm} / \mathrm{s}$ wiedergegeben. Zusätzlich ist das Spektrum des Hydrofonsignals dargestellt.

Das Spektrum der Wandgeschwindigkeit in Anwesenheit des Hydrofons zeigt das Auftreten der höheren Harmonischen der sowie einiger zusätzlicher Linien, die 


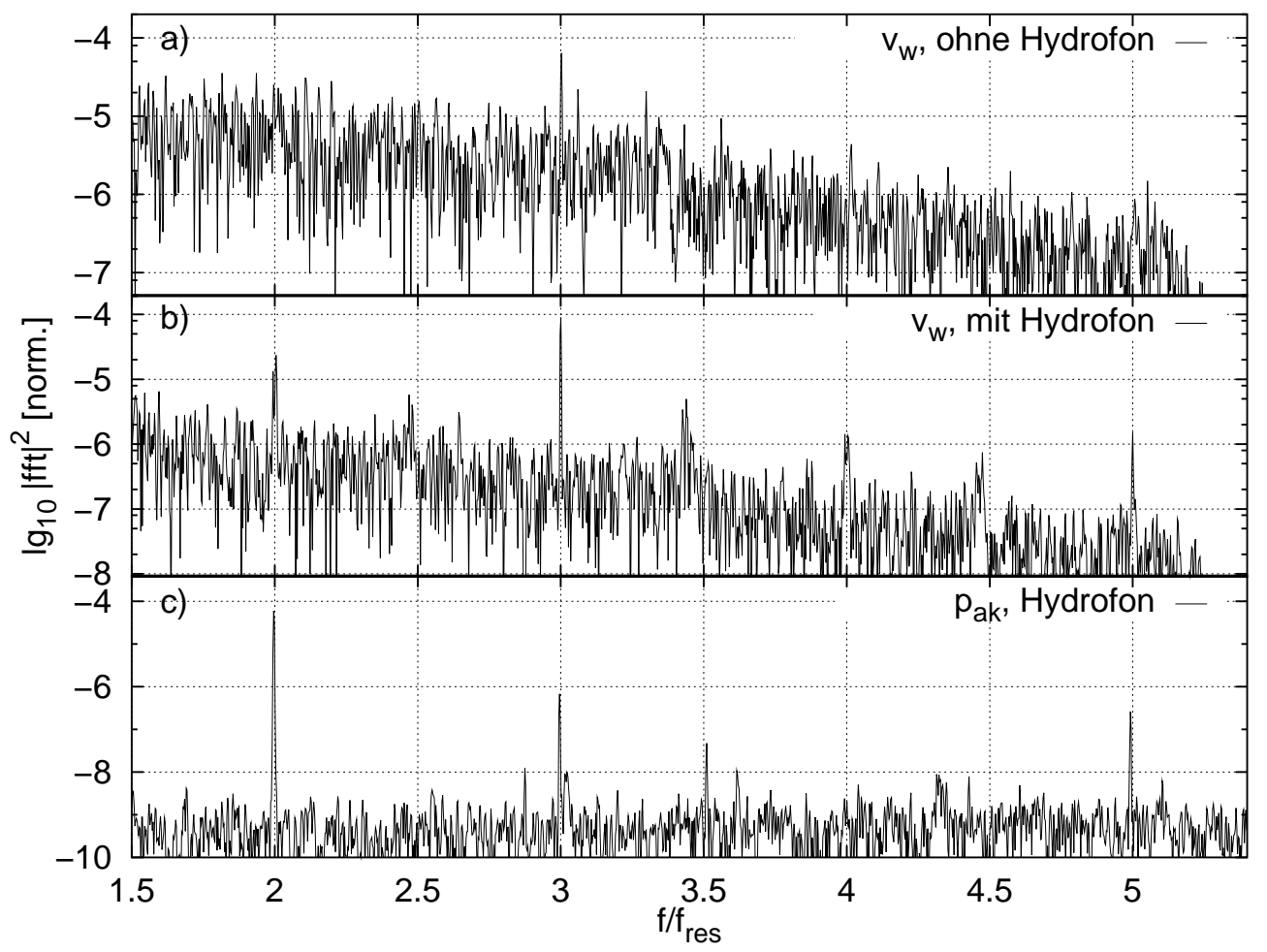

Abbildung A.4.: Ausschnitt der auf die Resonanzfrequenz $f_{\text {res }}$ normierten Leistungsspektren des Vibrometersignals ohne Hydrofon (a), mit Hydrofon (b) und des Hydrofonsignals (c) bei vergleichbarer Geschwindigkeitsamplitude $(0.8 \mathrm{~mm} / \mathrm{s})$.

möglicherweise Resonatormoden entsprechen. Die höheren Harmonischen sind vermutlich nicht durch Resonatormoden angeregt. Wegen der nichtidealen Randbedingungen des Resonators rechnet man mit einem frequenzabhängigen Einfluss der begrenzenden Masse (Glas und Piezoring) auf die Resonatoreigenschaften. Dabei wirkt die stärkere Dämpfung durch die Randmaterialien [77, 54] einer Erhöhung der Resonanzfrequenzen aufgrund der bei höheren Frequenzen abnehmenden virtuellen Grenzschicht [35] entgegen, die Lage der Moden ist also theoretisch schwer zu bestimmen. Das Spektrum des Hydrofons zeigt vor allem eine ausgeprägte zweite Harmonische. Erstaunlich ist das Auftreten der dritten Harmonischen auch in Abwesenheit des Hydrofons mit einer vergleichbaren Amplitude von $1 \%$ der Grundfrequenz. Für die Anregung der Harmonischen können Nichtlinearitäten in der Schwingung des Schallwandlers, der Verklebung, der Wand oder des Vibrometersignals selbst verantwortlich sein. Die genaue Ursache lässt sich anhand der Datenlage nicht klären. Im Wesentlichen beobachtet man bei steigender Anregungsamplitude eine Erhöhung des Signal-Rauschverhältnisses, so dass 
bei höheren Amplituden auch die fünfte Harmonische im Spektrum sichtbar wird. Die höheren Harmonischen nehmen aber im Vergleich zur Anregungsfrequenz monoton ab. Bei höheren Anregungsamplituden fallen diese Nichtlinearitäten also weniger stark ins Gewicht.

\section{Resonanzen der Küvette}

Um die Resonanzen der Küvette zu bestimmen, wird diese mit einem Frequenzwobbelsignal angeregt und die Wandschwingung mit einem 8-bit Oszilloskop aufgezeichnet.

Niederfrequente Resonanzen Aufgrund nichtidealer Randbedingungen werden auch Resonanzen unterhalb der (1,1,1)- Mode angeregt. Da dieser Bereich besonders bei der Auswertung des akustischen Spektrums von Bedeutung ist, wird simultan zum Vibrometersignal das Signal eines in einer oberen Ecke der Küvette angebrachten Hydrofons (Bruel \& Kjaer 8301) aufgezeichnet. In Abbildung A.5 sind die Resonanzkurven bei zwei verschiedenen Wasserständen wiedergegeben. Die Resonanzkurven in Bild a) sind bei einem Wasserstand von etwa $5 \mathrm{~mm}$ unterhalb der Küvettenoberkante aufgenommen, für die Messung in Bild b) ist die Küvette bis zum Rand gefüllt. Zum Vergleich ist in Bild c) die Wandgeschwindigkeit in Abwesenheit des Hydrofons bei ansonsten gleichen Bedingungen wie in b) wiedergegeben.

Die niederfrequente Resonanz bei etwa $4.5 \mathrm{kHz}$ ist unabhängig von der Füllhöhe. Als mögliche Ursache kommen Biegeschwingungen der Glaswände in Frage, deren Grundmode in der Größenordnung dieser Frequenz liegt (auch hier ist eine exakte Berechnung wegen der Randbedingungen schwierig). Die höherfrequenten Moden sind füllhöhenabhängig. Wird der Resonator in diesen Resonanzen betrieben, entstehen Kavitationsblasen in der Mode um 15-16 kHz zuerst in den unteren Ecken der Küvette, in der Mode um 17-18 kHz bilden sich am Boden vier Blasenzentren symmetrisch auf den Diagonalen. Bei höherer Anregung findet Kavitationsaktivität in den Kanten des Resonators und in der Mitte der Seitenflächen statt. Auch diese Moden sind also offenbar vornehmlich durch Wandschwingungen verursacht. Interessanterweise ist die Resonanz bei $15-16 \mathrm{kHz}$ in Anwesenheit des Hydrofons an der Stelle der Vibrometermessung stark gedämpft. Bei näherer Betrachtung der Daten sind im Vibrometersignal bei niedrigem (hohem) Wasserspiegel noch Linien bei 3.4(2.6), 6.4(5.3), 8.6(8.5), 10.2, 11.5(10.4) und $12.5 \mathrm{kHz}$ auszumachen. 


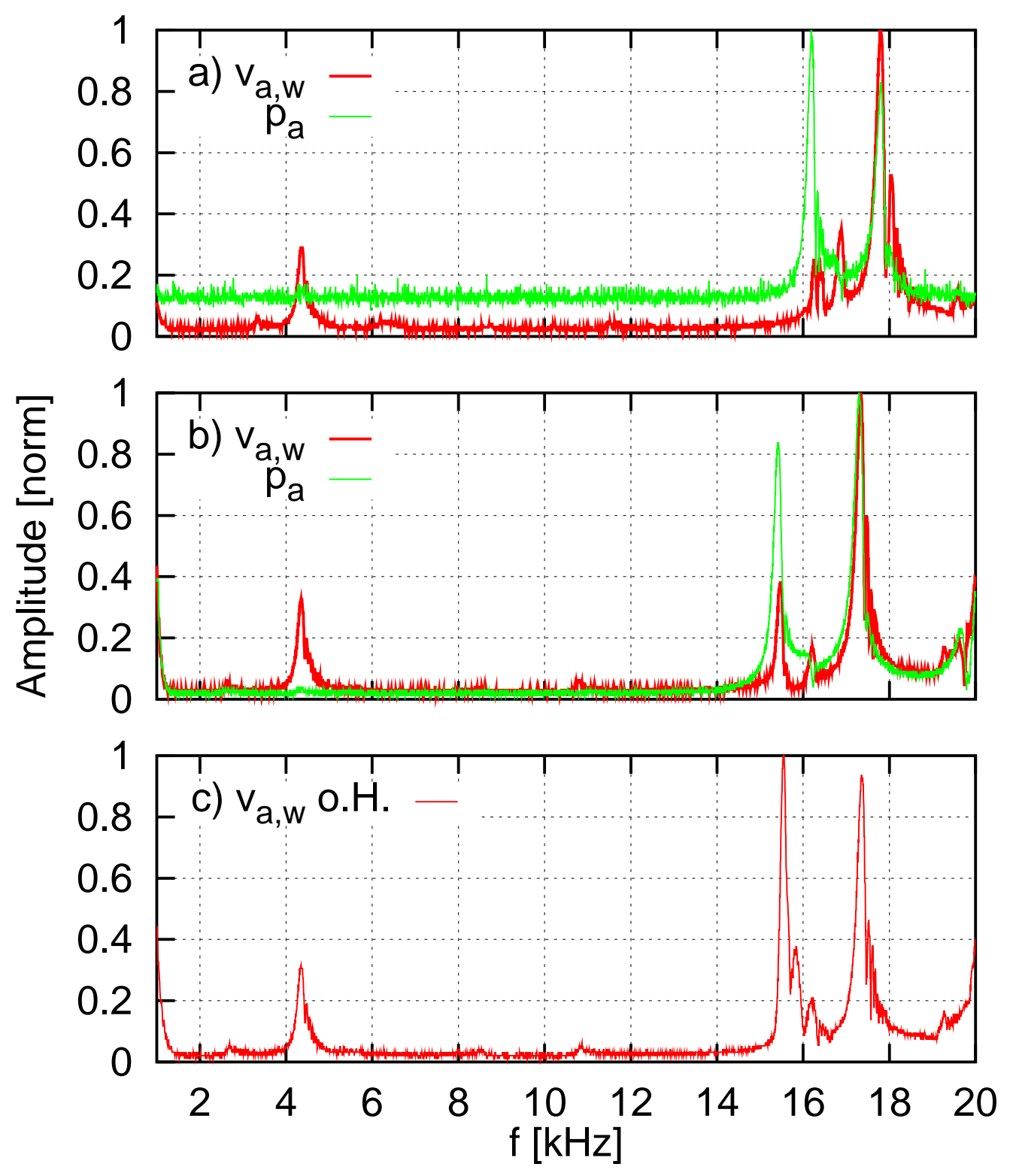

Abbildung A.5.: Resonanzkurven der Wandgeschwindigkeit (rot) und des Schalldrucks in einer oberen Ecke (grün) im niederfrequenten Bereich. Wasserspiegel: $5 \mathrm{~mm}$ unter Maximum (a), Maximum (b,c), wobei (c) in Abwesenheit des Hydrofons. 


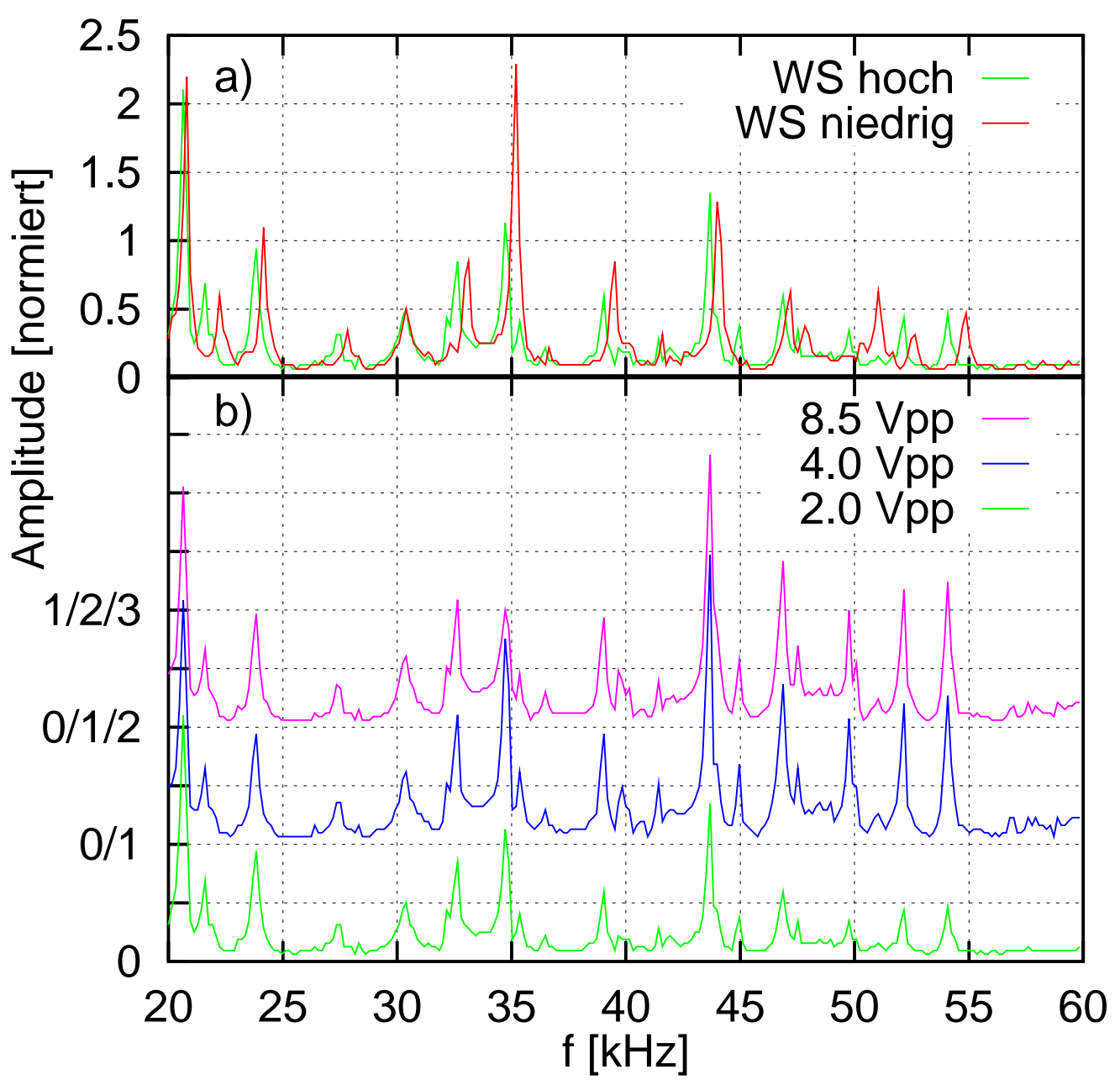

Abbildung A.6.: Resonanzkurven der Wandgeschwindigkeit in Abhängigkeit vom Wasserstand (WS, a), und der Anregungsamplitude (b). Die Amplituden sind auf die (1,1,1)-Mode bei hohem Wasserstand normiert.

Resonanzen im höherfrequenten Bereich Neben der Resonanz der (1,1,1)Mode schwingen noch eine Reihe anderer Moden an. Abbildung A.6 gibt die Resonanzkurven für zwei verschiedene Wasserstände (a) sowie für verschiedene Anregungsamplituden bei konstantem Wasserstand (b) wieder. Wie zu erwarten, verschieben sich die Resonanzen bei höherem Wasserstand zu niedrigeren Frequenzen. Normiert wurde hier auf die (1,1,1)-Mode bei hohem Wasserstand. Bei den starken Resonanzüberhöhungen handelt es sich vermutlich um Moden, bei denen die Wände stark ausgelenkt werden. Insbesondere die Resonanz na- 
he $20 \mathrm{kHz}$ zeigt eine sehr hohe Amplitude. Wie bei den niederfrequenten Moden, setzt auch hier Kavitation vergleichsweise früh an der Wand ein. Es gibt ebenfalls eine Resonanz (bei $\mathrm{f}=30 \mathrm{kHz}$ ), die nicht füllstandabhängig ist. Der Vergleich der Resonanzen bei unterschiedlicher Anregungsamplitude, die jedoch alle unter der Kavitationsschwelle liegen, zeigt das unterschiedlich starke Anschwingen der Moden im Vergleich zur (1,1,1)-Mode. Vor allem Resonanzen oberhalb $\mathrm{f}=40 \mathrm{kHz}$ werden bei hoher Anregungsamplitude stärker angeregt. Abbildung A.7 zeigt die Resonanzkurven im anschließenden Frequenzbereich von 60 bis $100 \mathrm{kHz}$, bei einer Anregungsamplitude von 2 Vpp. Die Normierung ist wie bei Abbildung A.6. Bemerkenswert ist hier das starke Anschwingen der dritten Harmonischen um $\mathrm{f}=72 \mathrm{kHz}$.

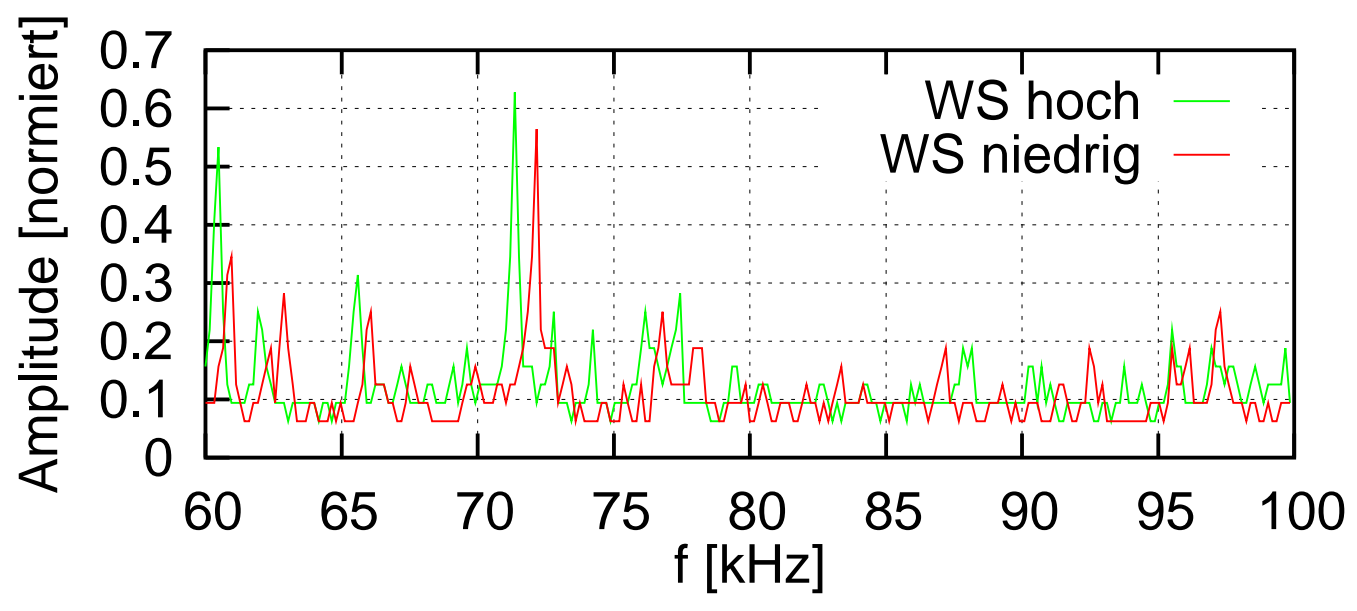

Abbildung A.7.: Resonanzkurven der Wandgeschwindigkeit in Abhängigkeit vom Wasserstand (WS).

Insgesamt zeigt sich ein reichhaltiges Modenspektrum. Neben aus den Volumenschwingungen resultierenden Moden sind Resonanzen, die durch Oberflächenschwingungen verursacht werden, messbar. 


\section{A.2. Plexiglasquader}

\section{A.2.1. Beschreibung des Resonators}

Als Resonator wird eine selbstgebaute quaderförmige Küvette verwendet (R. Geisler und J. Appel). Die Wände bestehen aus PMMA, der Boden ist wegen der besseren Wärmebeständigkeit aus Polycarbonat gefertigt. Die Wände und der Boden sind mit Acrifix (Firma Röhm) verklebt, als Schallwandler wurde eine scheibenförmige Piezokeramik (pi ceramics, PIC 155) am Resonatorboden befestigt (Uhu endfest 300). Die Abmessungen der Bauteile betragen:

1. Küvette

Innenmaße: $\mathrm{x}=\mathrm{y}=47 \mathrm{~mm}, \mathrm{z}=61 \mathrm{~mm}$

Wandstärke: $2 \mathrm{~mm}$

2. Piezokeramik

Durchmesser: $47 \mathrm{~mm}$

Höhe: $12 \mathrm{~mm}$

Aufgrund der dicken, den Boden fast komplett bedeckenden Piezokeramik lässt sich der Küvettenboden nicht mehr als schallweicher Abschluss idealisieren. In erster Näherung verhält er sich vielmehr wie ein schallharter Abschluss, so dass sich am Küvettenboden ein Schalldruckmaximum ausbildet. In Abbildung A.9 ist die normierte Schalldruckamplitude in Abhängigkeit von der Anregungsfrequenz $f_{0}$ und der Höhe $\mathrm{z}$ wiedergegeben. $\mathrm{z}=0$ bezeichnet hier den Küvettenboden, bei $\mathrm{z}=5 \mathrm{~cm}$ befindet sich der Wasserspiegel. 


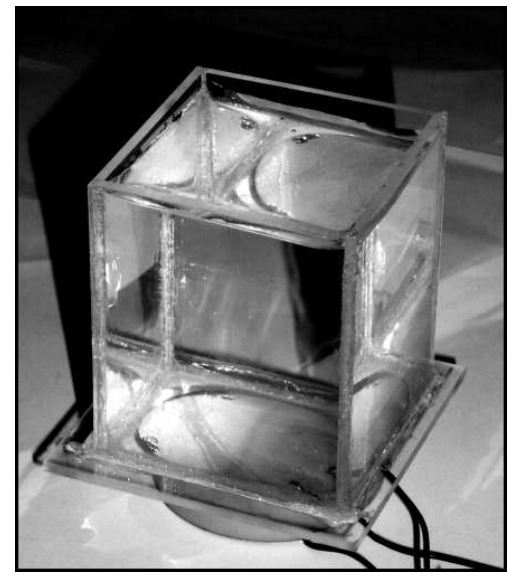

Abbildung A.8.: Aufnahme des Plexiglasquaders. Aufnahme J. Appel.

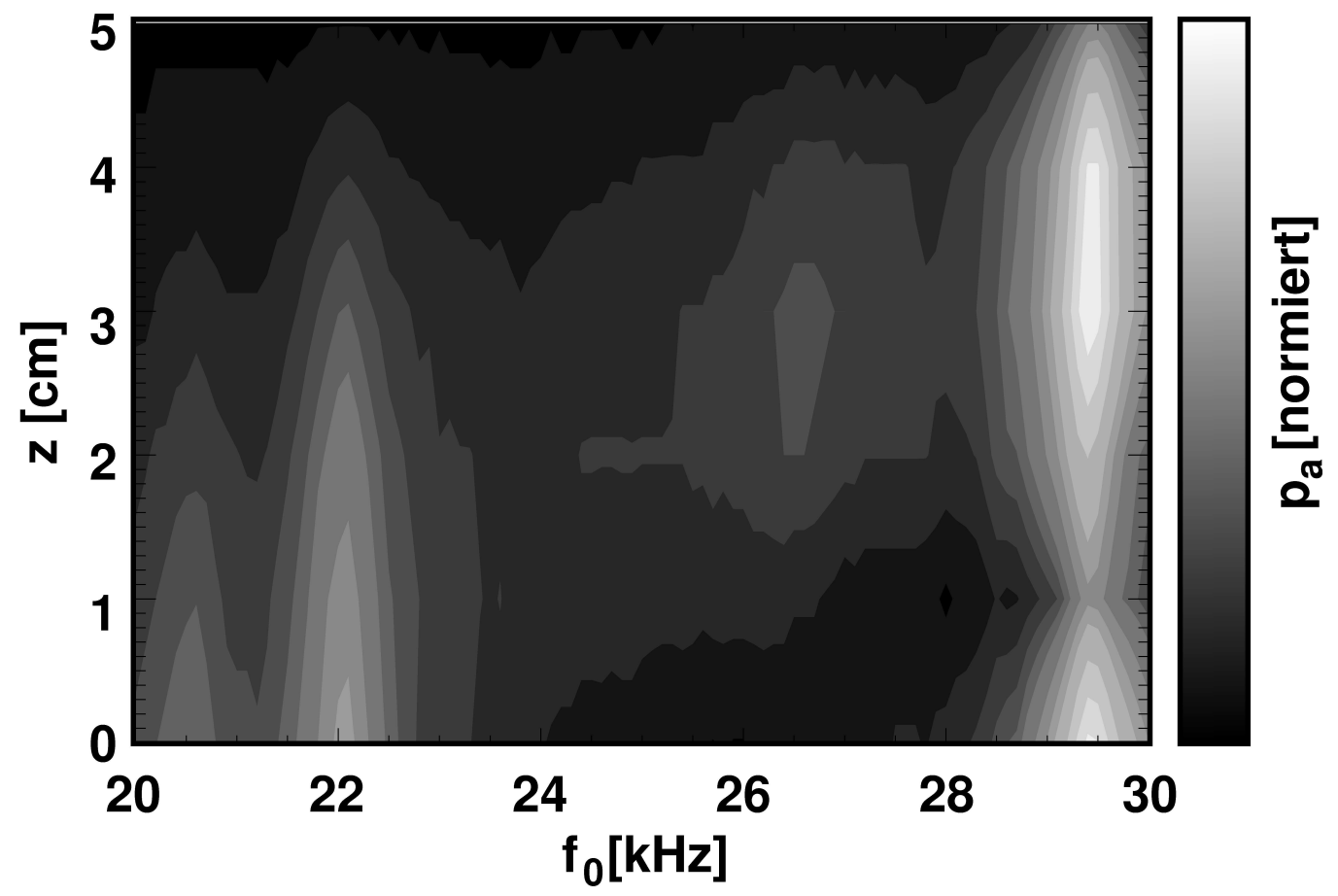

Abbildung A.9.: Vertikale Schalldruckverteilung im Plexiglasquader im niederfrequenten Ultraschall. $\mathrm{z}=0$ bezeichnet den Küvettenboden, die Wasseroberfläche befindet sich bei $\mathrm{z}=5 \mathrm{~cm}$. 


\section{A.3. Plexiglaswanne}

\section{A.3.1. Beschreibung des Resonators}

Für Versuchszwecke wurde uns eine Testwanne der Firma Elma zur Verfügung gestellt. Sie besteht aus einer Wanne aus PMMA, an deren Unterseite zwei Leistungsschallwandler verschraubt und verklebt sind. Die Abmessungen der Wanne betragen:

Innenmaße: $\mathrm{x}=150 \mathrm{~mm}, \mathrm{y}=80 \mathrm{~mm}, \mathrm{z}=125 \mathrm{~mm}$

Wandstärke: $5.0 \mathrm{~mm}$

Die Wandler haben zwei Resonanzfrequenzen $\left(f_{\text {res }, 1}=27 \mathrm{kHz}, f_{\text {res }, 2}=45 \mathrm{kHz}\right)$. Sie können einzeln mit beliebigem Signal angesteuert werden. Abbildung A.10 zeigt ein Foto der Wanne.

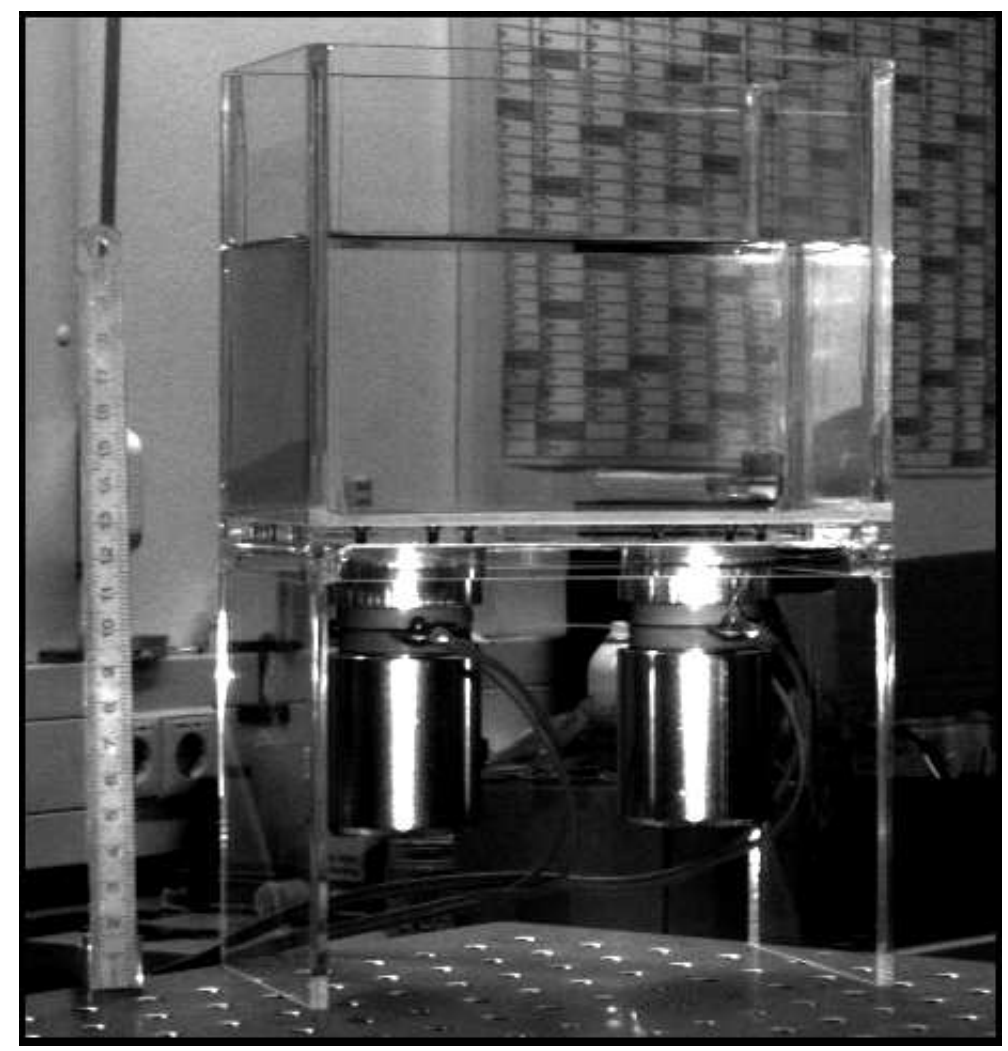

Abbildung A.10.: Die Plexiglaswanne. 


\section{A.4. Ultraschallreaktor}

Für Untersuchungen in starken Schallfeldern stand uns ein industrieller Ultraschallreaktor zu Verfügung (ELAC nautic LVG 61-02MOD). Die Schallwandler sind unzugänglich in die Bodenplatte aus Stahl eingebaut, deshalb können keine näheren Angaben dazu gemacht werden. Die Seitenwände wurden gegen PMMAScheiben ausgetauscht. Die Abmessungen der Wanne betragen

Innenmaße: $\mathrm{x}=190 \mathrm{~mm}, \mathrm{y}=155 \mathrm{~mm}, \mathrm{z}=200 \mathrm{~mm}$

Wandstärke: $5 \mathrm{~mm}$

Bei einer angegebenen maximalen Leistung von $1000 \mathrm{~W}$, einem elektrischen Konversionsfaktor von 0.95 und einem akustischen Konversionsfaktor von 0.75 liegt die maximale akustische Leistung bei etwa $700 \mathrm{~W}$.

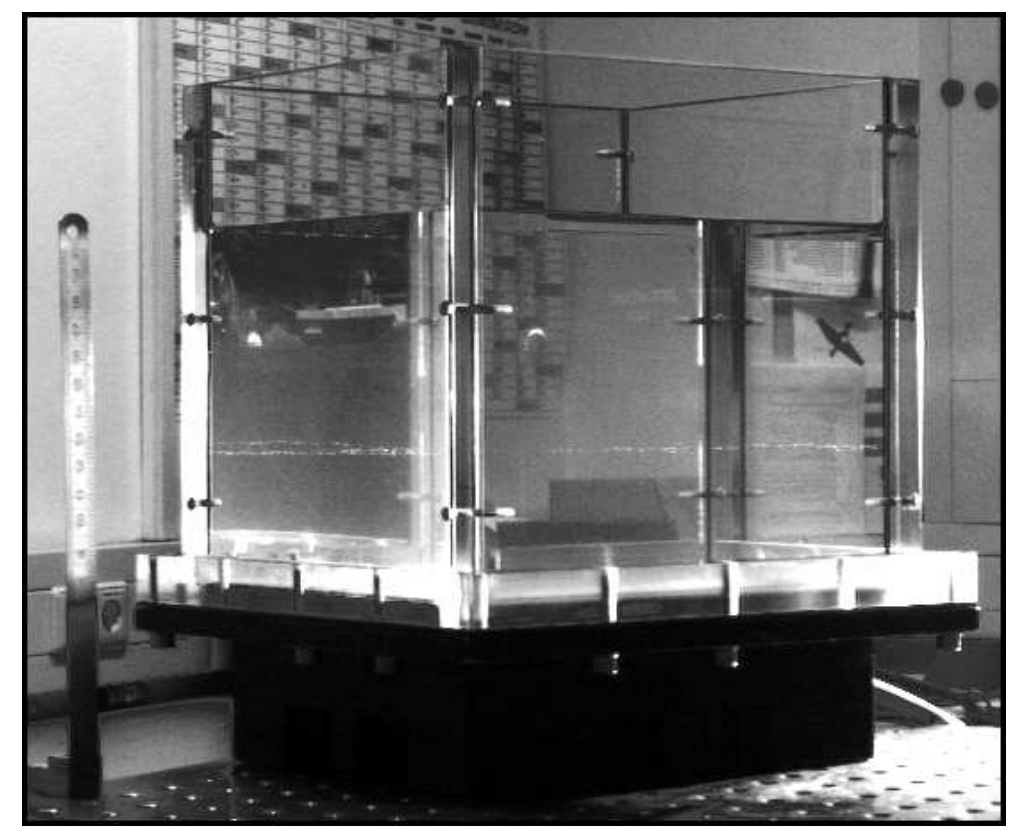

Abbildung A.11.: Bild des Ultraschallreaktors.

Der Reaktor wird über einen mitgelieferten Verstärker bei einer Frequenz von etwa $40 \mathrm{kHz}$ angesteuert, die im Rahmen von etwa $100 \mathrm{~Hz}$ automatisch leistungsangepasst wird. Zudem ist das Signal mit der Netzfrequenz amplitudenmoduliert. Die Leistung kann in 5\% Schritten verändert werden, minimale Leistungsstufe ist dabei $15 \%$. Bereits bei niedrigster Leistungsstufe ist die Kavitationsschwelle überschritten, so dass eine blasenfreie Messung des Schallfeldes nicht möglich ist. 
Die Schalldruckamplituden zu bestimmen ist wegen der Störung durch Kavitationsblasen problematisch. Abbildung A.12 zeigt die Maximal- und Effektivwerte zweier bei einer Leistungsstufe von 15\% aufgenommenen Schalldruckmessungen. Zusätzlich ist der an den Effektivwert angefittete Schalldruckverlauf eines reinen Stehwellenfeldes eingezeichnet.

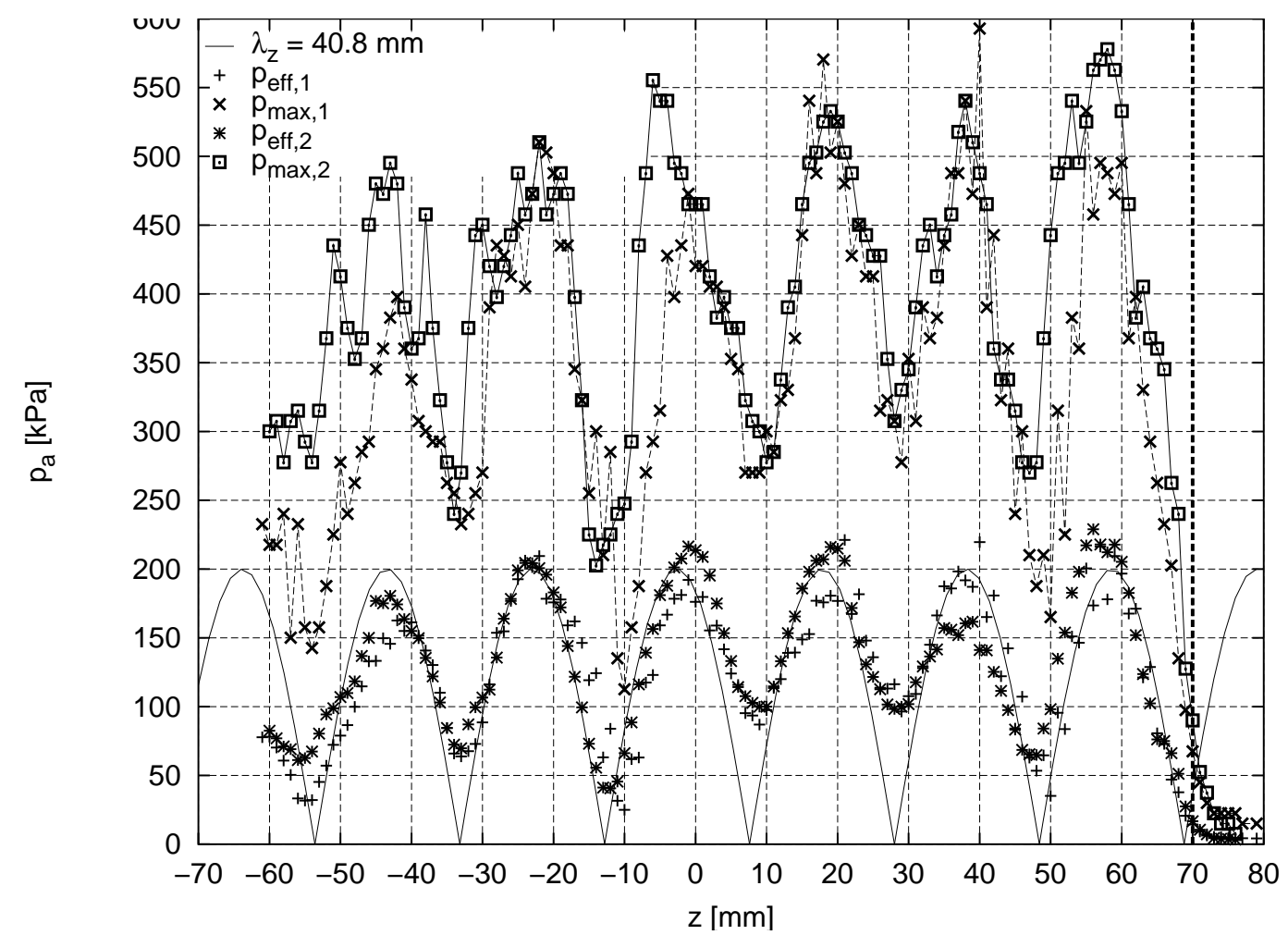

Abbildung A.12.: Schalldruckverteilung des Ultraschallreaktors auf der z-Achse. Leistungstufe: 15\%, Ursprung: Zentrum des Reaktors, Bodenplatte: $\mathrm{z}=-70 \mathrm{~mm}$, Wasseroberfläche: $\mathrm{z}=70 \mathrm{~mm}$.

Die maximalen Schalldruckamplituden schwanken erheblich. Eine Messreihe dauert etwa 30 Minuten. In dieser Zeit erhöht sich die Wassertemperatur um etwa $1^{\circ} \mathrm{C}$. Da der Temperaturanstieg auch noch nach Abschalten des Reaktors messbar ist und die Bodenplatte sehr warm wird, sind für die Temperaturerhöhung vermutlich in erster Linie Biegeschwingungen der Platte verantwortlich. Die räumlichen Maxima der Schalldruckamplitude sind trotzdem reproduzierbar. Aus dem Abstand zwischen zwei Schalldruckbäuchen bzw. -knoten folgt

$$
\lambda_{z}=(40.8 \pm 2) \mathrm{mm}
$$

Für einen ideal schallharten Boden und eine ideal schallweiche Wasseroberfläche ergäbe sich als Resonatorbedingung $\lambda_{z}=42.14 \mathrm{~mm}$. Für eine eindimensionale 
ebene Welle von $40 \mathrm{kHz}$ in Wasser ist die Wellenlänge $\lambda=37.05 \mathrm{~mm}$, sie ist also nur wenig kleiner als die gemessene Wellenlänge in z-Richtung. In $\mathrm{x}$ und $\mathrm{y}$ Richtung können also nur noch recht niedrige Moden mit vergleichsweise großer Wellenlänge die Resonatorbedingung erfüllen. Der Schalldruckverlauf auf der xund y-Achse ist in Abbildung A.13 wiedergegeben. Hier gibt es jeweils nur zwei
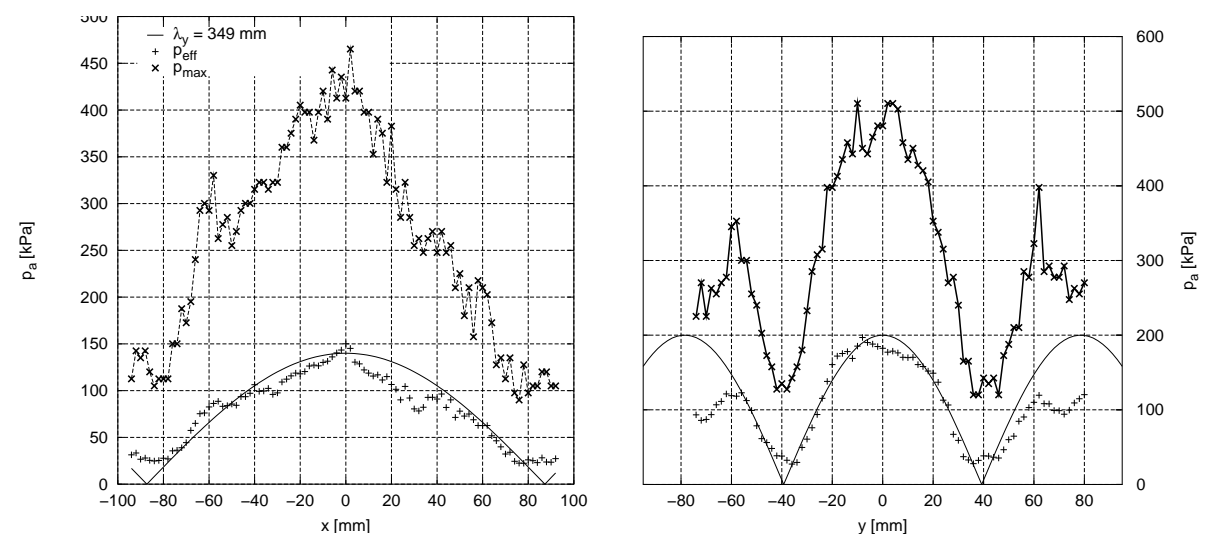

Abbildung A.13.: Schalldruckverteilung des Ultraschallreaktors auf der $\mathrm{x}$-Achse (links) und $\mathrm{y}$-Achse (rechts), Leistung $15 \%$.

Schalldruckminima, in x-Richtung ist nahezu die Grundmode angeregt. Das Stehwellenfeld ist im Wesentlichen geprägt durch die Anordnung und Abstrahlung der Schallwandler und deren Reflexion an der Wasseroberfläche und nicht durch die Resonatoreigenschaften des Wasservolumens. Bemerkenswert ist die nichtverschwindende Schalldruckamplitude in den Minima. Dies bedeutet, dass Wirkleistung in den Reaktor gebracht wird, also ein beträchtlicher Anteil an gerichteter Wellenausbreitung stattfindet. 


\section{Anhang B}

\section{Symbole}

Ich habe mich bemüht, alle Symbole für jeweils nur eine Variable oder Konstante zu verwenden. Da viele Variablen-Namen im üblichen Gebrauch doppelt belegt sind (z.B. T für Temperatur und Periodendauer), weichen einige Variablenbezeichnungen von den üblicherweise oder in den zitierten Veröffentlichungen benutzten ab. In dieser Liste sind zur Übersicht noch einmal alle verwendeten Symbole mit ihren Bedeutungen aufgeführt. Die Symbole sind alphabetisch und nach Groß- und Kleinschreibung sortiert. 
$\underline{A} \quad$ Koeffizientenvektor in der Lösung der Laplaceschen Gleichung

$C \quad$ Schallgeschwindigkeit

$C(r) \quad$ Korrelationssumme

$D \quad$ Federkonstante eines linearen Oszillators

$D_{\text {corr }} \quad$ Korrelationsdimension

E Kinetische Energie

F $\quad$ Kraft

$F_{0} \quad$ Amplitude einer periodischen Kraft

$F_{B} \quad$ Hydrodynamische Kraft auf die Blase

$F_{D} \quad$ Rückstellkraft

$F_{b j 1} \quad$ Primäre (erste) Bjerkneskraft

$F_{b j 2} \quad$ Sekundäre (zweite) Bjerkneskraft

$F_{g} \quad$ Gravitationskraft

$F_{h s} \quad$ Auftriebskraft

$F_{i} \quad$ Kraft zwischen Partikeln im Aggregationsmodell

$F_{k} \quad$ Reibungskraft

$N \quad$ Anzahl der Nachbarn im Aggregationsmodell

$N_{x_{k}} \quad$ Anzahl der Nachbarn des Referenzpunktes $x_{k}$ in $C(r)$

$P \quad$ Impuls

$R(t) \quad$ Blasenradius

$\dot{R} \quad$ Radialgeschwindigkeit der Blasenwand

$R_{0} \quad$ Ruheradius einer Blase im statischen Gleichgewicht

$R_{a} \quad$ Amplitude einer linearen Radiusschwingung

$R_{B} \quad$ Kritischer Blakeradius

$R_{a t} \quad$ Kleine Auslenkung aus der Ruhelage einer Blase

$R_{r} \quad$ Resonanzradius

$T \quad$ Periodendauer $\frac{2 \pi}{\omega}$

$T_{x} \quad$ Temperatur in der Blase

$U \quad$ Elektrische Spannung

$U_{a} \quad$ Spannungsamplitude

$U_{a, F G}$ Spannungsamplitude der Funktionsgenerators

$U_{a, p z} \quad$ Spannungsamplitude am Schallwandler

$V \quad$ Volumen

$V_{0} \quad$ Volumen einer Blase mit dem Ruheradius $R_{0}$

$X_{l} \quad$ Molenbruch der Flüssigkeit 
c $\quad$ Parameter der Korrekturfunktion $\mathrm{f}(\mathrm{c})$

$c_{x} \quad$ Konzentration des Gases $\mathrm{x}$ in der Flüssigkeit

$c_{x, 0} \quad$ Sättigungskonzentration des Gases $\mathrm{x}$ in der Flüssigkeit

$c_{x, \infty} \quad$ Gleichgewichtskonzentration des Gases $\mathrm{x}$ in der Flüssigkeit

$f \quad$ Frequenz

$f(c) \quad$ Korrekturfunktion bei der Mittelung der Translation

$f_{t_{0}} \quad$ Startfrequenz

$f_{\text {res }} \quad$ Resonanzfrequenz des blasenfreien Systems

$f_{r e s, b}$ Resonanzfrequenz des Systems bei Anwesenheit einer Blase

$f_{\text {res, } h}$ Resonanzfrequenz des Systems bei Anwesenheit des Hydrofons

$f_{0} \quad$ Anregungsfrequenz bei Spektralanalyse

$f_{b j} \quad$ Proportionalitätsfaktor der primären Bjerkneskraft

$g \quad$ Schwerebeschleunigung $\approx 9.81 \frac{\mathrm{m}}{\mathrm{s}^{2}}$

$h \quad$ Tiefe innerhalb einer Flüssigkeit $(\mathrm{h}=0$ : Wasseroberfläche)

$k_{b} \quad$ Boltzmann-Konstante

$h_{x} \quad$ Henrykonstante des Gases $\mathrm{x}$ in der Flüssigkeit (temperaturabhängig)

$k \quad$ Dämpfungskonstante eines linearen Oszillators

$k \quad$ Potenz des Kraftgesetzes im Aggregationsmodell

$k \quad$ Wellenzahl

$k_{x} \quad$ Wellenzahl in x-Richtung

$m \quad$ Masse

$m_{l} \quad$ Molekülmasse der Flüssigkeit

$m_{v} \quad$ Masse der Flüssigkeit

$n_{R} \quad$ Anzahl der Referenzpunkte bei der Bestimmung von $C(r)$

$n_{D} \quad$ Anzahl der Datenpunkte bei der Bestimmung von $C(r)$

$p_{0} \quad$ Standarddruck: $100 \mathrm{kPa}$

$p_{a} \quad$ Schalldruckamplitude

$p_{a k} \quad$ Schalldruck

$p_{R} \quad$ Blaseninnendruck

$p_{B} \quad$ statische Blakeschwelle

$p_{R, v} \quad$ Partialdruck der Flüssigkeit in der Blase

$p_{R, x} \quad$ Partialdruck eines Stoffes in der Blase

$p_{h s} \quad$ Hydrostatischer Druck

$p_{v} \quad$ Dampfdruck

$p_{\infty} \quad$ Druck in der Flüssigkeit in großer Entfernung von der Blase

$p_{\sigma} \quad$ Durch Oberflächenspannung erzeugter Druck auf die Blasenwand 
$r \quad$ Radialkoordinate des äußeren Bezugssystems in Kugelkoordinaten

$t \quad$ Zeit

$v \quad$ Geschwindigkeit $=\dot{x}$

$v_{w} \quad$ Wandgeschwindigkeit des Resonators (Vibrometersignal)

$v_{a, w} \quad$ Geschwindigkeitsamplitude der Resonatorwandschwingung

$v_{T} \quad$ Mittlere Translationsgeschwindigkeit einer Blase

$x_{B} \quad$ Ort des Blasenmittelpunktes im äußeren Bezugssystem

$x_{k} \quad$ Referenzpunkt bei der Bestimmung von $C(r)$

$\Phi \quad$ Geschwindigkeitspotential

$\gamma \quad$ Polytropenexponent eines Gases

$\eta \quad$ Dynamische Zähigkeit (Viskosität) einer Flüssigkeit

$\nu \quad$ Frequenz

$\rho \quad$ Dichte

$\sigma \quad$ Oberflächenspannung einer Flüssigkeit

$\sigma_{r r}$ Radialkomponente des zähen Spannungstensors

$\phi \quad$ Phasendifferenz zwischen Blasenschwingung und Anregung

$\omega \quad$ Kreisfrequenz der Anregung: $2 \pi \nu$

$\omega_{0} \quad$ Eigenfrequenz eines Resonators 


\section{Literaturverzeichnis}

[1] I. Akhatov, N. Gumerov, C.D. Ohl, U. Parlitz und W. Lauterborn: The role of surface tension in stable single-bubble sonoluminescence. Phys. Rev. Lett. 78(2), 227-230, 1997. 64

[2] I. Akhatov, R. Mettin, C.D. Ohl, U. Parlitz und W. Lauterborn: Bjerknes force threshold for stable single bubble sonoluminescence. Phys. Rev. E 55(3), 3747-3750, 1997. 79

[3] J. Appel: Stereoskopische Messung von Blasentrajektorien in akustischen Stehwellenfeldern. Diplomarbeit, Universität Göttingen, 2002. 77

[4] G.A. Askar'yan, A.M. Prokhorov, G.F. Chanturiya und G.P. Shipulo: The effects of a laser beam in a liquid. Soviet Physics JETP. 17(6), 1963. 6

[5] T. Barbat, S. Ashgriz und C. Liu: Dynamics of two interacting bubbles in an acoustic field. J. Fluid. Mech. 389, 137-168, 1999. 13

[6] B.P. Barber, K.R. Weniger, R. Löfstedt und S.J. Putterman: Observation of a new phase of sonoluminscence at low partial pressures. Phys. Rev. Lett. $\mathbf{7 4}, 5276-5279,1996.63,64,66$

[7] L. Bergmann: Der Ultraschall, Kap. Weitere Anwendungen des Ultraschalls, S. 709-790. Hirzel Verlag, Stuttgart, 6. Aufl., 1954. 5

[8] W.H. Besant: Hydrostatics and Hydrodynamics. Deighton Bell, Cambridge, 1859. 9

[9] V. Bjerknes: Vorlesungen über hydrodynamische Fernkräfte nach C. A. Bjerknes' Theorie, Bd. 2, Kap. 1. Verlag von Johann Ambrosius Barth, Leibzig, 1902. 4, 12, 24

[10] F.G. Blake: The onset of cavitation in liquids. Techn. Ber. 12, I. Acoustics Res. Lab., Harvard Univ., 1949. 77 
[11] www-zeus.physik.uni bonn.de: . 4

[12] C.E. Brennen: Cavitation and Bubble Dynamics. Nr. 44 in Oxford Engineering sciene series, Oxford University Press, 1995. 3

[13] M. Brenner, D. Lohse, D. Oxtoby und T.F. Dupont: Mechanisms for stable single bubble sonoluminescence. Phys. Rev. Lett. 76(7), 1158-1161, 1996. 66

[14] M.P. Brenner, S. Hilgenfeldt und D. Lohse: Single-bubble sonoluminescence. Rev. Mod. Phys 74(2), 2002. 62

[15] E. Cramer: Numerische und experimentelle Untersuchungen zum Kavitationsgeräusch. Dissertation, Universität Göttingen, 1978. 107

[16] L.A. Crum: Bjerknes forces on bubbles in a stationary sound field. J. Acoust. Soc. Am. 57(6), 1363-1370, 1975. 12, 14, 15, 24

[17] L.A. Crum: Measurements of the growth of air bubbles by rectified diffusion. J. Acoust. Soc. Am. 68(1), 203-211, 1980. 18

[18] L.A. Crum: The polytropic exponent of gas contained within air bubbles pulsation in a liquid. J. Acoust. Soc. Am. 73(1), 116-120, 1983. 49

[19] L.A. Crum und A.I. Eller: Motion of bubbles in a stationary sound field. J. Acoust. Soc. Am. 48(1), 181-189, 1970. 12, 14, 15, 37, 46

[20] L.A. Crum und D.A. Nordling: Velocity of transient cavities in an acoustic stationary wave. J. Acoust. Soc. Am. 42(1), 294-301, 1972. 98

[21] L.A. Crum und A. Prosperetti: Nonlinear oscillations of gas bubbles in liquids: An interpretation of some experimental results. J. Acoust. Soc. Am. 73(1), 121-127, 1983. 37

[22] A.A. Doinikov: Translational motion of a spherical bubble in an acoustic standing wave of high intensity. Phys. Fluids 14(4), 1420-1425, 2002. 12, 14,15

[23] A. Eller: Force on a bubble in a standing acoustic wave. J. Acoust. Soc. Am. 43(1), 170-171, 1968. 49

[24] A. Eller und L.A. Crum: Instability of the motion of a pulsating bubble in a sound field. J. Acoust. Soc. Am. 47(3), 762-767, 1970. 37, 56 
[25] A. Eller und H.G. Flynn: Rectified diffusion during nonlinear pulsations of cavitation bubbles. J. Acoust. Soc. Am. 37(3), 493-503, 1965. 20

[26] A. Eller und H.G. Flynn: Growth of bubbles by rectified diffusion. J. Acoust. Soc. Am. 46(5), 1246-1250, 1969. 40

[27] P.S. Epstein und M.S. Plesset: On the stability of gas bubbles in liquid-gas solutions. J. Chem. Phys. 18, 1505-1509, 1950. 19

[28] R. Esche: Untersuchung der Schwingungskavitation in Flüssigkeiten. Acustica, Beih. 2(4), 208-218, 1952. 107

[29] H. Fischer und H. Kaul: Mathematik für Physiker, Bd. 1. B.G.Teubner, Stuttgart, 2. Aufl., 1990. 27

[30] T. Fließbach: Elektrodynamik, Kap. Kugelfunktionen, S. 113-118. B.I. Wissenschaftsverlag, 1994. 21

[31] M.M. Fyrillas und A.J. Szeri: Dissolution or growth of soluble spherical oscillation bubbles. J. Fluid Mech. 277, 381-407, 1994. 20

[32] D.F. Gaitan, L.A. Crum, C.C. Church und R.A. Roy: Sonoluminescence and bubble dynamics for a single, stable, cavitation bubble. J. Acoust. Soc. Am. 91(6), 3166-3183, 1992. 6

[33] D.F. Gaitan und R.G. Holt: Experimental observation of bubble response and light intensity near the threshold for single bubble sonoluminescence in an air-water system. Phys. Rev. E 59(5), 5495-5502, 1999. 56, 62, 63

[34] Pennsylvania State University Garfield Thomas Water Tunnel: Knapp,Daily,Hammit:Cavitation, 1970. 4

[35] R. Geisler: Akustische Blasenfallen für die Sonolumineszenz. Diplomarbeit, Universität Göttingen, 1998. 144

[36] R. Geisler: Universität Göttingen, 2003. 4

[37] C. Gerthsen, H. Kneser und H. Vogel: Physik. Springer-Verlag, 16. Aufl., 1992. 11

[38] F.R. Gilmore: The growth or collapse of a spherical bubble in a viscous compressible liquid. Hydrodynamics Laboratory Report No.26-4, California Institute of Technology , 1952. 10 
[39] R.K. Gould: Rectified diffusion in the presence of, and absence of, acoustic streaming. J. Acoust. Soc. Am. 56(6), 1740-1747, 1974. 56, 59

[40] P. Grassberger und I. Procaccia: Characterization of strange attractors. Phys. Rev. Lett. 50(5), 346-349, 1983. 102

[41] A. Harkin, T.J. Kaper und A. Nadim: Coupled pulsation and translation of two gas bubbles in a liquid. J. Fluid Mech. 445, 377-411, 2001. 13

[42] G. Haussmann: Zeitabhängigkeit des Geräuschspektrums bei Schwingungskavitation. Diplomarbeit, Universität Göttingen, 1967. 107

[43] R.A Hiller und B.P. Barber: Producing light from a bubble of air. Scientific American S. 78-80, Feb. 1995. 62

[44] R.G. Holt und D.F. Gaitan: Observation of stability boundaries in the parameter space of single bubble sonoluminescence. Phys. Rev. Lett. 77, 3791-3794, 1996. 37, 62, 66

[45] J. Holzfuss: Zur Messung von fraktalen Dimensionen und LyapunovSpektren nichtlinearer dynamischer Systeme am Beispiel akustisch erzeugter Kavitationsblasenfelder. Dissertation, Universität Göttingen, 1987. 107

[46] J. Holzfuss, M. Rüggeberg und R.G Holt: Sonolumineszenz: Instabile Diffusion und chemische Reaktionskinetik. In: Fortschritte der Akustik DAGA 2001, Dega e.V., Oldenburg, 2001. 68

[47] J. Holzfuss, M. Rüggeberg und R. Mettin: Boosting sonoluminescence. Phys. Rev. Lett. 81(9), 1961-1964, 1998. 37

[48] D. Hsieh und M. Plesset: Theory of rectified diffusion of mass into gas bubbles. J. Acoust. Soc. Am. 33(2), 206-215, 1961. 20

[49] J.B. Keller und M. Miksis: Bubble oscillations of large amplitude. J. Acoust. Soc. Am. 68(2), 628-633, 1980. 10

[50] J.A. Ketterling und R.E. Apfel: Experimental validation of the dissociation hypothesis for single bubble sonoluminescence. Phys. Rev. Lett. 81(22), 4991-4994, 1998. 37

[51] J.A. Ketterling und R.E. Apfel: Extensive experimental mapping of sonoluminescence parameter space. Phys. Rev. E 61, 3832-3837, 2000. 63 
[52] R.T. Knapp, J.W. Daily und F.G. Hammit: Cavitation. McGraw-Hill Book Company, 1970. 3

[53] P. Koch: Simulation der Bewegung akustischer Kavitationsblasen durch ein Partikelmodell. Diplomarbeit, Universität Göttingen, 2001. 60

[54] D. Krefting: Einzelblasensonolumineszenz bei Zweifrequenzanregung. Diplomarbeit, Universität Göttingen, 1999. 144

[55] D. Krefting, R. Mettin und W. Lauterborn: Two-frequency driven singlebubble sonoluminescence. J. Acoust. Soc. Am. 112, 1918-1927, 2002. 63

[56] D. Krefting, R. Mettin und W. Lauterborn: Single-bubble sonoluminescence in air-saturated water. Phys. Rev. Lett. zur Veröffentlichung akzeptiert, 2003. 62

[57] H. Kuttruff: Physik und Technik des Ultraschalls. Hirzel Verlag, Stuttgart, 1988. 5

[58] Sir H. Lamb: Hydrodynamics, Kap. Oscillations of a viscous sphere, S. 641. Cambridge University Press - London, 6. Aufl., 1932. 9, 21, 64

[59] L.D. Landau und E.M. Lifschitz: Hydrodynamik. Nr. 6 in Lehrbuch der Theoretischen Physik, Akademie Verlag, Berlin, 5. Aufl., 1991. 13

[60] W. Lauterborn: Resonanzkurven von Gasblasen in Flüssigkeiten. Acustica 23, 73-81, 1970. 107

[61] W. Lauterborn: High-speed photography of laser-induced breakdown in liquids. J. Appl. Phys. 21(1), 27-29, 1972. 6

[62] W. Lauterborn: Numerical investigation of nonlinear oscillations of gas bubbles in liquids. J. Acoust. Soc. Am. 23, 283-293, 1976. 107

[63] W. Lauterborn, Hg.: Cavitation and Inhomogeneities in Underwater Acoustics, Bd. 4 von Springer Series in Electrophysics. Springer Verlag, Heidelberg, 1980. 3, 4

[64] W. Lauterborn und E. Cramer: Subharmonic route to chaos observed in acoustics. Phys. Rev. Lett. 47(20), 1445-1448, 1981. 107

[65] W. Lauterborn und A. Koch: Holographic observation of period-doubled and chaotic bubble oscillations in acoustic cavitation. Phys. Rev. A 35, 1974-1976, 1987. 121 
[66] T.G. Leighton: The Acoustic Bubble. Academic Press, 1994. 4, 11

[67] Gnu General Public Licence: TSTOOL V1.11 - MATLAB-Implementation. www.physik3.gwdg.de/tstool/indexde.html. 103

[68] D. Lohse, M.P. Brenner, T.F. Dupont, S. Hilgenfeldt und B. Johnston: Sonoluminescing air bubbles rectify argon. Phys. Rev. Lett. 78, 1359-1362, 1997. 64

[69] J.W.S. Lord Rayleigh: On the pressure developed in a liquid during the collapse of a spherical cavity. Phil. Mag. 34, 94-98, 1917. 4, 9

[70] O. Louisnard und F. Gomez: Growth by rectified diffusion of strongly acoustically forced gas bubbles in nearly saturated liquids. Phys. Rev. E 67, 036610, 2003. 79

[71] S. Luther: Theoretische Beschreibung und experimentelle Untersuchung raum-zeitlicher Strukturbildung in akustischen Kavitationsfeldern. Dissertation, Universität Göttingen, 2000. 36, 71, 77, 79

[72] J. Magnaudet und D. Legendre: The viscous drag force on a spherical bubble with a time-dependent radius. Phys. Fluids 10(3), 550-554, 1998. 12, 13, $14,31,52$

[73] T.J. Matula, S.M. Cordry und L.A. Roy, R.A. Crum: Bjerknes force and bubble levitation under single-bubble sonoluminescence conditions. J. Acoust. Soc. Am. 102(3), 1522-1527, 1997. 37

[74] R. Mettin: 3. Physikalisches Institut, Universität Göttingen. 23

[75] R. Mettin, S. Luther, S. Kamphausen und W. Lauterborn: Dynamics of delay-coupled spherical bubbles. In: Nonlinear Acoustics at the Turn of the Millennium, herausgegeben von W. Lauterborn und T. Kurz, Nr. 524 in AIP Conference Proceedings, S. 359-362, 2000. 24

[76] R. Mettin, S. Luther, C.D. Ohl und W. Lauterborn: Acoustic cavitation structures and simulations by a particle model. Ultrasonics Sonochemistry 6, 25-29, 1999. 60

[77] E. Meyer und G. Neumann: Physikalische und Technische Akustik, Kap. Dämpfung von Schall, S. 86-103. F. Vieweg und Sohn, 1967. 144

[78] M. Minnaert: On musical air-bubbles and the sounds of running water. Phil. Mag. 16, 235-248, 1933. 11 
[79] E.A. Neppiras: Subharmonic and other low-frequency emission from bubbles in sound-irradiated liquids. J. Acoust. Soc. Am. 46(3), 587-601, 1969. 107

[80] B.E. Noltingk und E.A Neppiras: Cavitation produced by utrasonics. Proc. Phys. Soc. B63, 674-685, 1950. 9

[81] J. Olaf: Oberflächenreinigung mit Ultraschall. Acustica 7(5), 253-263, 1957. 125

[82] U. Parlitz, V. Englisch, C. Scheffczyk und W. Lauterborn: Bifurcation structure of bubble oscillators. J. Acoust. Soc. Am. 88(2), 1061-1077, 1990. 107

[83] U. Parlitz, R. Mettin, S. Luther, I. Akhatov, M. Voss und W. Lauterborn: Spatio-temporal dynamics of acoustic cavitation bubble clouds. Phil. Trans. R. Soc. Lond. A 357, 313-334, 1999. 12, 14, 15, 77

[84] M.S. Plesset: The dynamics of cavitation bubbles. J. Appl. Mech. 16, 277-282, 1949. 9

[85] M.S. Plesset: On the stability of fluid flows with spherical symmetry. J. Appl. Phys. 25, 96-98, 1954. 21, 22, 64

[86] M.S. Plesset und T.P. Mitchell: On the stability of the spherical shape of a vapor cavity in a liquid. Q. Appl. Math., 1956. 22

[87] S. Popinet und S. Zaleski: Coupling of radial and translational motion in small viscous bubbles. In: Proceeding of FEDSM99, 3dr ASME/JSME Joint Fluids Engineering Conference, Am. Soc. Mech. Eng., 1999. 12, 14, 15

[88] H. Poritsky: The collapse or growth of a spherical bubble or cavity in a viscous fluid. In: Proceedings of the First U.S. National Congress on Applied Mechanics, herausgegeben von E. Sternberg, S. 813-821, New York, 1952. 9

[89] A. Prosperetti: Thermal effects and damping mechanisms in the forced radial oscillations of gas bubbles in liquids. J. Acoust. Soc. Am. 61, 17-27, 1977. 49

[90] A. Prosperetti: Viscous effects on pertubated spherical flows. Q. Appl. Math. 35, 339-352, 1977. 22 
[91] A.J. Reddy und A.J. Szeri: Coupled dynamics of translation and collapse of acoustically driven microbubbles. J. Acoust. Soc. Am. 112(4), 1346-1352, 2002. $12,13,14,15,26,32$

[92] Inc. Research Systems: IDL version 5.5, solaris, 2001. 36

[93] L.D. Rozenberg: Physical Principles of ultrasonic technology, Bd. 1, Kap. Ultrasonic cleaning. Plenum Press, New York - London, 1973. 125

[94] C. Scheffczyk: Kavitationsblasendynamik. Dissertation, Universität Darmstadt, 1993. 105, 106

[95] H. Strube: Numerische Untersuchungen zur Stabilität nichtsphärisch schwingender Blasen. Acustica 25(5), 289-303, 1971. 64

[96] Inc. The MathWorks: MATLAB version 6.100.0.88, 2000. 36

[97] R. Toegel und D. Lohse: Phase diagrams for sonoluminescing bubbles: A comparison between experiment and theory. J. Chem. Phys. 118, 1863-1875, 2003. 65

[98] Inc. Visual Numerics: PV-WAVE cl version 6.10, 1997. 36, 50

[99] T. Watanabe und Y. Kukita: Translational and radial motions of a bubble in an acoustic standing wave field. Phys. Fluids A 5(11), 2682-2688, 1993. $13,14,15$

[100] F.R. Young: Cavitation. McGraw-Hill Book Company, 1989. 3 


\section{Danksagung}

Ich danke Herrn Prof. Dr. Werner Lauterborn für die Ermöglichung dieser Arbeit. Die interessanten Diskussionen, die hervorragende experimentelle Ausstattung, die unbürokratische Hilfe und das Vertrauen in die Eigenverantwortlichkeit waren eine solide Basis zur Erstellung dieser Arbeit. Sein kritischer Blick auf die Wirklichkeit hat mich oft ermutigt.

Dr. Robert Mettin danke ich für die durchgängige, kompetente Unterstützung der Arbeit, die wichtigen Anregungen und spannenden Diskussionen sowie die sorgfältige Durchsicht des Manuskripts. Insbesondere möchte ich mich dafür bedanken, mir durch die Übernahme der formalen Aufgaben des BMBF-Projektes die Zeit für die zahlreichen Experimente freigehalten zu haben.

Prof. Dr. Ulrich Parlitz habe ich die Erweiterung meiner Arbeit um den Aspekt der akustischen Spektren zu verdanken. Für die betreuerischen Tätigkeiten in diesem Bereich und die weit darüber hinausgehende Hilfe mit Rat und Tat insbesondere in der Endphase der Arbeit möchte ich mich an dieser Stelle bedanken.

Herrn Prof. Dr. Dirk Ronneberger danke ich für die Übernahme des Korreferats und die geduldige Hilfsbereitschaft bei der Interpretation diverser akustischer Phänomene.

Reinhard Geisler gilt mein Dank für die unschätzbare Hilfe bei den Experimenten, sowohl bei der Konzeption der Experimente als auch bei den alltäglichen Unwägbarkeiten des Laborlebens. Die Konstruktion des Glasresonators hat eine Reihe der Experimente erst möglich gemacht, seine GIMP-plug-ins haben die Erzeugung einer Reihe von Abbildungen in der vorliegenden Arbeit sehr vereinfacht. Darüber hinaus bedanke ich mich für die gemeinsame Zeit im Schreibraum, die selbst das Zusammenschreiben zu einem erträglichen Zustand hat werden lassen.

Für die Hilfe bei der Korrektur der Arbeit danke ich Andrea Pfitzner, Annette Fölster, Dennis Kröninger, Dirk Gros, Ingo Berentzen, Matthias Grott und Ole Hölck. Dinah Loerke und Reinhard Geisler danke ich für die gemeinsame Umsetzung des Projektes "Disseptember", das die Fertigstellung der Dissertation ungemein erleichtert hat.

Bei Dr. Stefan Luther bedanke ich mich für die viel zu kurze konstruktive Zusammenarbeit und die Gespräche über Physik und alles was dazugehört. 
Reinhard Sobotta und Dr. Christoph Jung von der Firma ELMA danke ich für die anregenden Diskussionen im Rahmen des BMBF-Projektes und die Erweiterung des Blickwinkels um die Sichtweise des Anwenders.

Matthias Grott bin ich für die vielen physikalischen Diskussionen und für die Hilfe bei der analytischen Lösung der Differentialgleichungen dankbar.

Der elektronischen und mechanischen Werkstatt des Instituts unter der Leitung von Dr. Karl Lautscham und Herrn Dieter Hille danke ich für die unbürokratische und prompte Hilfe bei der Realisierung der Experimente, sei es die Konstruktion von Spezialanfertigung, die schnelle Reparatur von defekten Geräten oder die Bereitstellung von werkstatteigenen Instrumenten für die Messungen.

Thomas Geiling, Dr.Hans-Werner Strube und Gerhard König gilt mein Dank für den reibungslosen Betrieb des Institutsnetzes, die Hilfe bei Rechnerproblemen und die Unterstützung bei der Erstellung der Datenbank.

Gisa Kirschmann-Schröder danke ich für fotografische Hilfe, für die anregenden Diskussionen, die mir oft eine neue Sicht der Dinge gegeben haben, und für alles andere. Bernadette Tyson, Elke Zech und Martina Schulz danke ich für die Hilfe bei der Überwindung bürokratischer Hürden und für Vieles, was das Leben im und ums Institut verschönert hat.

Betty Remstedt und Ilse Bleßmann danke ich dafür, dass sie selbst in der Endphase der Dissertation unseren Schreibraum in einen der Arbeit zuträglichen Zustand versetzt haben.

Die Arbeit wurde vom Graduiertenkolleg „Strömungsinstabilitäten und Turbulenz" und vom BMBF-Verbundprojekt „Untersuchung von Kavitationsfeldern“ finanziell unterstützt.

Den derzeitigen und ehemaligen Angehörigen der gesamten Arbeitsgruppe "Nichtlineare Dynamik" danke ich für die freundschaftliche Atmosphäre, insbesondere Gerrit Langer, Martin Voss, Tobias Brendel, Volker Ahlers, Stefan Kamphausen, Philipp Koch, Olgert Lindau, Thomas Kurz, Jörg D. Wichard, Christian Merkwirth und Lutz Junge.

Meinen Eltern Ernst-Rudolf und Friederike Krefting, meiner Großmutter Eva Krefting und meinem Onkel Hans Rindfleisch danke ich für die jahrelange Unterstützung, für das Interesse an meiner Arbeit und dafür, dass sie mich nicht nur mit Puppen, sondern auch mit der elektrischen Eisenbahn haben spielen lassen. 


\section{Lebenslauf}

Name: Dagmar Krefting

Geboren: 15.04.1972 in Münster

Eltern: Dr. Ernst-Rudolf und Dr. Friederike Krefting

$\begin{array}{ll}1978-1982 & \text { Paul-Gerhard-Grundschule Hiltrup } \\ 1982-1991 & \text { Immanuel-Kant-Gymnasium Hiltrup } \\ \text { Mai 1991 } & \text { Abitur } \\ 1991-1998 & \text { Studium der Chemie, Universität Göttingen. } \\ 1994-1999 & \text { Studium der Physik, Universität Göttingen. } \\ \text { Oktober 1999 } & \text { Diplom in Physik } \\ \text { November 1999 } & \text { Beginn der Promotion } \\ & \text { Stipendium des Graduiertenkollegs } \\ & \text { "Strömungsinstabilitäten und Turbulenz“ } \\ \text { Januar - August 2000 } & \text { Wissenschaftliche Tätigkeit an der } \\ & \text { Facultad de Ciencias, Instituto de Física } \\ & \text { in Montevideo, Uruguay } \\ \text { seit Februar 2001 } & \text { Wissenschaftliche Mitarbeiterin } \\ & \text { im Rahmen des BMBF-Projektes } \\ & \text { "Untersuchung von Kavitationsfeldern“ }\end{array}$

